\title{
World checklist of threatened mammals
}


A|N $5986-3$ 


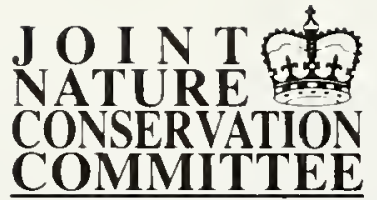

\section{World checklist of threatened mammals}

compiled by the

World Conservation Monitoring Centre

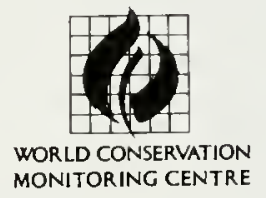

on behalf of the

United Kingdom Scientific Authority for Animals 
Prepared under contract from the Joint Nature Conservation Committee by the World Conservation Monitoring Centre, Cambridge, UK. Established in 1988 as a company limited by guarantee with charitable status, WCMC is managed as a joint-venture between the three partners in the World Conservation Strategy and its successor Caring For The Earth: IUCN - The World Conservation Union, UNEP - United Nations Environment Programme, and WWF - World Wide Fund for Nature. Its mission is to provide information on the status, security, management and utilisation of the world's biological diversity to support conservation and sustainable development.

Published by: Joint Nature Conservation Committee

Copyright: 1993 Joint Nature Conservation Committee

ISBN: $\quad$ 1st edition published 1987 ISBN 0-86139-336-8

2nd edition published 1993 ISBN 1-873701-44-6

Citation: World Conservation Monitoring Centre (1993). World checklist of threatened mammals. Joint Nature Conservation Committee, Peterborough.

Available from: Further copies and companion volumes for other animal species available from:

Natural History Book Service Ltd

2 Wills Road

Totnes

Devon TQ9 5XN

United Kingdom

Telephone (from UK): $\quad 0803865913$

Telephone (from outside UK): +44803865913

The designations of geographical entities in this book, and the presentation of the material, do not imply the expression of any opinion whatsoever on the part of JNCC or WCMC concerning the legal status of any country, territory, or area, or of its authorities, or concerning the delimitation of its frontiers or boundaries. 


\section{Contents}

Preface

Acknowledgements

Introduction

Explanatory notes

Introductory references

Taxonomic List

MONOTREMATA

Tachyglossidae

\section{MARSUPIALIA}

Dasyuridae

Thylacinidae

Myrmecobiidae

Peramelidae

Thylacomyidae

Phalangeridae

Petauridae

Burramyidae

Vombatidae

Macropodidae

\section{INSECTIVORA}

Solenodontidae

Tenrecidae

Potamogalidae

Chrysochloridae

Erinaceidae

Soricidae

Talpidae

\section{MACROSCELIDEA}

Macroscelididae

\section{CHIROPTERA}

Pteropodidae

Emballonuridae

Craseonycteridae

Megadermatidae

Rhinolophidae

Phyllostomidae

Myzopodidae

Vespertilionidae

Mystacinidae

Molossidae

\section{PRIMATES}

Tupaiidae

Lemuridae

Cheirogaleidae

Indriidae

Daubentoniidae

Lorisidae
Galagidae $\quad 24$

Tarsiidae 25

Callithricidae 25

Callimiconidae 27

Cebidae 27

Cercopithecidae 31

Hylobatidae 41

Pongidae 41

\section{EDENTATA}

Myrmecophagidae $\quad 42$

Bradypodidae 43

Choloepidae 43

Dasypodidae 43

\section{PHOLIDOTA}

Manidae

\section{LAGOMORPHA}

Ochotonidae

Leporidae

\section{RODENTIA}

Aplodontidae $\quad 46$

Sciuridae 46

Geomyidae $\quad 48$

Heteromyidae 48

Castoridae 49

Anomaluridae 49

Cricetidae 49

Arvicolidae 51

Muridae 51

Zapodidae 53

Hystricidae 53

Erethizontidae 53

Dinomyidae 54

Agoutidae $\quad 54$

Dasyproctidae $\quad 54$

Chinchillidae 54

Capromyidae $\quad 54$

Capromyidae $\quad 55$

Octodontidae $\quad 55$

\section{CETACEA}

Platanistidae $\quad 55$

Ziphiidae 56

Physeteridae 58

Monodontidae 58

Delphinidae 59

Phocoenidae 63

Eschrichtidae 64

Balaenopteridae 64

Balaenidae 65 


\title{
CARNIVORA
}

Canidae

Ursidae

Procyonidae

Mustelidae

Viverridae

Herpestidae

Protelidae

Hyaenidae

Felidae

\section{PINNIPEDIA}

Otariidae

Odobenidae

Phocidae

\section{PROBOSCIDEA}

Elephantidae

HYRACOIDEA

Procaviidae

\section{SIRENIA}

Dugongidae

Trichechidae

\section{PERISSODACTYLA}

\author{
Equidae
}

Tapiridae

Rhinocerotidae

ARTIODACTYLA

Suidae

89

Tayassuidae

90

Hippopotamidae

Camelidae

90

Tragulidae

91

Cervidae

91

Bovidae

91

95

REFERENCES 


\section{Preface}

This checklist is one of a serjes prepared for the United Kingdom's Scientific Authority for Animals to assist in implementing the Convention on International Trade in Endangered Species of Wild Fauna and Flora (CITES). Similar checklists are available for other animal taxa.

The aim of this volume is to provide a summary of basic information on all species of mammals in CITES or appearing in the IUCN Red List of Threatened Animals, compiled by The World Conservation Monitoring Centre. The inclusion of scientific synonyms, English vernacular names and a full list of the countries in which each species occurs will provide a valuable quick source of reference for those assessing applications for CITES permits. The inclusion of many references in the text will permit access to more detailed information on the status of species in particular countries of origin.

Together these features will combine to make this volume of interest to all those concerned with the distribution and status of threatened or potentially threatened species whether from a professional or an amateur point of view.

The Joint Nature Conservation Committee is most grateful to the World Conservation Monitoring Centre for preparing this work on its behalf.

\section{Lord Selborne}

Chairman, Joint Nature Conservation Committee

Monkstone House

City Road

Peterborough

PE1 1JY

United Kingdom

\section{Acknowledgements}

This checklist was compiled by the World Conservation Monitoring Centre under contract F71-12-56 from the Joint Nature Conservation Committee. The authors were Tim lnskipp and Crawford Allan.

The volume builds on an earlier edition and the numerous contributors to that edition are acknowledged, particularly the authors, Tim Inskipp and Jonathan Barzdo.

John A. Burton is thanked for providing access to additional information sources.

For their assistance in producing the current edition, the following WCMC staff are also thanked: Duncan Bennet, Esther Byford, Lorraine Collins, Mary Cordiner, Helen Corrigan, Neil Cox, Brian Groombridge, Vanessa Heywood, Richard Luxmoore, Chris Magin, Julie Reay, Lindsay Simpson and Lucy Smith.

David Morgan of the JNCC International Policy Branch is thanked for providing expert advice and guidance throughout.

Timothy H. Johnson

Head, Species Unit

World Conservation Monitoring Centre

219 Huntingdon Road

Cambridge

CB3 ODL

United Kingdom 


\section{Introduction}

In April 1991, the Nature Conservancy Council for England, Countryside Council for Wales and Scottish Natural Heritage acting together through the Joint Nature Conservation Committee were appointed by the Secretary of State for the Environment as the United Kingdom's Scientific Authority for Animals under the Convention on International Trade in Endangered Species of Wild Fauna and Flora (CITES). CITES regulates international trade in wild animals and plants and in products derived from them, to help to ensure their conservation on a worldwide scale. The purpose of CITES is to regulate international trade in endangered wild animals and plants and in products derived from them, to help to ensure their conservation on a worldwide scale.

The purpose of this work is to provide a combined list of the species and subspecies included in Appendices I, II and III to the Convention on International Trade in Endangered Species of Wild Fauna and Flora (CITES), and those included in the current edition of the IUCN Red List of Threatened Animals. This document incorporates additions and amendments to the CJTES appendices up to and including those made at the 8 th Conference of the Parties in Kyoto in March 1992 (effective from 11 June 1992). The Red List is supplemented, in the case of mammals, by the later IUCN Red Data Book covering Cetacea (Klinowska, 1991). For each taxon, the scientific name is given first, with the currently most frequently used synonyms in brackets. Common names appear on the following lines below which the geographical range is indicated. The numbers appearing in square brackets refer to entries in the reference list, at the back of this document.
For background material on the rationale of the IUCN threat categories, readers are referred to IUCN (1990); for background on the workings of CITES, recommended works are Favre (1989) and Wijnstekers (1992). For information by country on the diversity and status of mammals and other taxonomic groups, and for a general review of biodiversity, readers are referred to WCMC (1992).

In a publication of this nature, it is inevitable that users will discover entries that need correcting or updating. The publishers would be grateful if their attention could be drawn to these entries, and the World Conservation Monitoring Centre would be grateful to receive details of such changes so that they can he incorporated in the datahase from which this publication is produced. 


\section{Explanatory notes}

\section{Scientific names}

In general, nomenclature of mammals follows (Honacki et al., 1982). However, where this differs from the nomenclature used in the Appendices to CITES, the latter has taken priority.

The taxonomic scope and sequence of orders in this checklist follow the system adopted in the Appendices to CITES. The Appendices are based on the classification of mammals proposed by Simpson (1945), but differs at ordinal level in separating Pinnipedia from Carnivora.

The taxonomic scope of families and their sequence within orders also follows the Appendices to CITES. The Appendices differ from Simpson (1945) at family level as given below:

The following families have been separated:

\section{Marsupialia}

Myrmecobiidae and Thylacinidae from Dasyuridae

Thylacomyidae from Peramelidae

Burramyidae and Petauridae from Phalangeridae

Primates

Cheirogaleidae from Lemuridae

Galagidae from Lorisidae

Callimiconidae from Cebidae

Hylobatidae from Pongidae

Edentata

Choloepidae from Bradypodidae

Rodentia

Arvicolidae from Cricetidae

Carnivora

Protelidae from Hyaenidae

The following families have been renamed:

\section{Marsupialia}

Phascolomidae now Vombatidae

Cetacea

$$
\text { Rhachianectidae now Eschrichtidae }
$$

The following families/genera have been included within another family:

\section{Chiroptera}

Hipposideridae in Rhinolophidae

Camivora

Ailuropoda in Ursidae not Procyonidae

Artiodactyla

Antilocapridae in Bovidae
The nomenclature of genera and species follows Honacki et al. except in the following instances:

1. Tamandua mexicana is included in $T$. tetradactyla

2. Coendou mexicanus is listed as Sphiggurus mexicanus

3. Stenella plagiodon is included with Stenella frontalis under the latter name

4. Ursus thibetanus is listed as Selenarctos thibetanus

5. Lynx canadensis is listed as Felis canadensis

6. Lynx caracal is listed as Felis caracal

7. Lynx lynx is listed as Felis lynx

8. Lynx pardinus is listed as Felis pardina

9. Lynx rufus is listed as Felis rufa

10. Equus asinus is listed as $E$. africanus

11. Equus kiang and $E$. onager are included in $E$. hemionus

12. Equus przewalskii is separated from $E$. caballus

13. Bubalus bubalis is listed as $B$. arnee

14. Tragelaphus eurycerus is listed as Boocercus eurycerus

15. Bos frontalis is listed as $B$. gaurus

16. Bos grunniens is listed as $B$. mutus

17. Bubalus bubalis is listed as $B$. arnee

18. Ovis ophion is listed as $O$. orientalis ophion rather than $O$. musimon ophion or $O$. aries ophion.

These differences are included as synonyms in the list, and alternative names used in (Corbet and Hill, 1991) are also included as synonyms.

\section{English names}

The common names used are taken from Corbet and Hill (1991), from the IUCN Red Data Book series and from a number of standard reference works for the mammals of particular regions. Secondary common names have been included wherever this was considered useful, including non-English names commonly used by English speakers. For the Cetacea, the common names used in Watson (1981), have also been included. 


\section{Other information}

The three columns headed CITES, RL and Ref, list the following information for each taxon.

\section{CITES}

I, II or III in this column refers to the appendix in which the taxon is listed under the Convention on International Trade in Endangered Species of Wild Fauna and Flora. The two-letter abbreviations following Appendix III entries denote the countries whose governments have placed the taxon in this appendix, as follows:

$\begin{array}{ll}\text { BW } & \text { Botswana } \\ \text { CA } & \text { Canada } \\ \text { CR } & \text { Costa Rica } \\ \text { GH } & \text { Ghana } \\ \text { GT } & \text { Guatemala } \\ \text { HN } & \text { Honduras } \\ \text { IN } & \text { India } \\ \text { NP } & \text { Nepal } \\ \text { TN } & \text { Tunisia } \\ \text { UY } & \text { Uruguay }\end{array}$

RL

Threat categories follow those given in the 1990 IUCN Red List and are as follows:

E Endangered

V Vulnerable

R Rare

$\mathrm{K}$ Insufficiently known

Id Indeterminate (given as "I" in RDB, but modified here to avoid confusion with the CITES "I" entry).

- not listed: note that this includes species which have not yet been evaluated for threatened status as well as those which have been evaluated and found not to be threatened

The threat categories for all Cetacea taxa listed are taken from Klinowska (1991).

\section{References}

The numbers in the column on the far right refer to an entry in the reference list at the back of this document. References may contain information on the status, distribution or nomenclature of the taxon in question. References which refer to the taxon in only a limited part of its range are generally excluded from this column and, instead, included in square brackets at the appropriate place/s within the geographical range information.

\section{Geographical Range}

The geographical range of each taxon is given in terms of political units, listed in alphabetical order.

When a taxon is found in a restricted area within a larger political unit, the name of the smaller area is given in brackets, e.g. Indonesia (Kalimantan).

When the name of a country is given as within the range of a certain taxon, that taxon may or may not occur throughout the country, and may even occur only peripherally.

Without surveying the range of each taxon in the field, it is necessary to rely on published records. Most taxonomic works give the range of a taxon in terms of broad geographical areas, rather than of political units. While some countries have a relatively well-known and well-reported mammalian fauna, some others do not. These factors may occasionally have resulted in some of the geographical ranges given here being incomplete or inaccurate, although every effort has been made to prevent this.

For Cetacea, geographical ranges are first expressed in terms of oceans, seas, and rivers where the tax on is known to occur or to have occurred. Published sources may not distinguish live and dead cetacean records, but all records have been included. Most cetaceans are widespread but coasts of some countries within their overall range may be avoided for physical, chemical or ecological reasons. Only confirmed sightings are included and taxa may occur in political units other than those named within their overall range.

A question mark '?' has been used in front of a country to indicate that there is some uncertainty in the status of the species in that country.

A country where the species is known to be extinct is denoted by '(ex)', or by '(ex?)' where there is still a small possibility that the species survives, or where recent searches have been unsuccessful.

'I ]' denotes a range state where the species has been introduced. 
This checklist has been made as compact as possible by listing names of countries and dependent territories in the shorter forms given by United Nations (1991). Within the text, Czechoslovakia and Yugoslavia are used to denote the geographic areas as recognised by United Nations (1991). However, changes to the political boundaries within the former USSR have been incorporated in the geographic descriptions.

Countries for which shorter forms of names are used and their shortened form:

Brunei Darussalam

Democratic People's Republic of Korea

Falkland Islands and Dependencies

Islamic Republic of Jran

Lao People's Democratic Republic

Libyan Arab Jamahiriya

Northern Mariana Islands

Republic of Korea

Saint Vincent and the Grenadines

Syrian Arab Republic

United Republic of Tanzania

United Kingdom of Great Britain and Northern lreland

United States of America
Brunei

D.P.R. Korea

Falkland Islands

Iran

Lao P.D.R.

Libya

Northem Marianas

Korea Republic

Saint Vincent

Syria

Tanzania

United Kingdom

USA

In the text, parent countries of dependencies are ommitted. These are listed below:

American Samoa, USA

Anguilla, United Kingdom

Aruba, Netherlands

Azores, Portugal

Bermuda, United Kingdom

Bouvet Island, Norway

British Antarctic Territory, United Kingdom

British Indian Ocean Territory, United Kingdom

British Virgin Islands, United Kingdom

Canary Islands, Spain

Canton and Enderbury Islands, Kiribati

Cayman Islands, United Kingdom

Channel Islands, United Kingdom

Christmas Island, Australia

Cocos Keeling Islands, Australia

Cook Islands, New Zealand

Falkland Islands, United Kingdom

Faeroe Islands, Denmark

Federated States of Micronesia, USA

French Guiana, France

French Polynesia, France

French Southern and Antarctic Territories, France

Galapagos Islands, Ecuador

Gibraltar, United Kingdom

Greenland, Denmark

Guadeloupe, France

Guam, USA

Guernsey, United Kingdom

Hawaiian Islands, USA

Heard and Macdonald Islands, Australia

Hong Kong, United Kingdom

Isle of Man, United Kingdom
Jersey, United Kingdom

Johnston Atoll, USA

Macao, Portugal

Macquarie Island, Australia

Madeira, Portugal

Marshall Islands, USA

Martinique, France

Mayotte, France

Montserrat, United Kingdom

Netherlands Antilles, Netherlands

New Caledonia, France

Niue, New Zealand

Norfolk Island, Australia

Northem Marianas, USA

Palau, USA

Panama Canal Zone, USA

Pitcaim Islands, USA

Puerto Rico, USA

Queen Maud Land, Norway

Réunion, France

Saint Helena, United Kingdom

Saint Pierre and Miquelon, France

South Orkney Islands, United Kingdom

South Sandwich Islands, United Kingdom

South Georgia, United Kingdom

South Shetland lslands, United Kingdom

Svalbard and Jan Mayen Islands, Norway

Tokelau, New Zealand

Tristan da Cunha Islands, United Kingdom

Turks and Caicos Islands, United Kingdom

Virgin Islands of the United States, USA

Wallis and Futuna, France 


\section{Introductory References}

Corbet, G.B. and Hill, J.E. 1991. A world list of mammalian species. (3rd edition). Natural History Museum Publications. Oxford University Press, Oxford.

Favre, D.S. 1989. Intemational trade in endangered species: $a$ guide to CITES. Martinus Nijhoff, Dordrecht.

Honacki, J.H., Kinman, K.E. and Koeppl, J.W. 1982. Mammal species of the world, a taxonomic and geographic reference. The Association of Systematics Collections, Lawrence, Kansas.

IUCN. 1990. 1990 IUCN red list of threatened animals. IUCN, Gland and Cambridge.

Klinowska, M. 1991. Dolphins, porpoises and whales of the world: the IUCN red dasa book. IUCN, Gland and Cambridge.
Simpson, G.G. 1945. The principles of classification and a classification of mammals. Bulletin of the American Museum of Natural History 85: 1-350.

United Nations. 1991. Terminology bulletin na. 342. United Nations, New York.

Watson, L. 1981. Sea guide to whales of the world. Hutchinson, London.

Wijnstekers, W. 1992. The evolution of CITES. A reference to the Convention on Intemational Trade in Endangered Species of Wild Fauna and Flora. (3rd revised edition). CITES Secretariat, Lausanne.

World Conservation Monitoring Centre. 1992. Global biodiversity: staus of the earh's living resaurces. Chapman and Hall, London 


\section{Class: MAMMALIA}

\section{Order MONOTREMATA}

Family TACHYGLOSSIDAE

Zaglossus bruijni (Peters \& Doria 1876)

II

V $\quad 1098$

Long-beaked Echidna, Long-nosed Echidna

Indonesia: Irian Jaya; Papua New Guinea

\section{Order MARSUPIALIA}

Family DASYURIDAE

Antechinus apicalis (Gray 1842)

ld $\quad 1044,1098$

(= Parantechinus apicalis)

Dibbler

Australia

Phascogale calura Gould 1844

ld $\quad 1044,1098$

Red-tailed Phascogale, Red-tailed Wambenger

10

Australia

Sminthopsis douglasi Archer 1979

ld $\quad 1044,1098$

Julia Creek Dunnart

Australia

Sminthopsis longicaudata Spencer 1909

Long-tailed Dunnart, Long-tailed Sminthopsis

Australia

Sminthopsis psammophila Spencer 1895

\section{Sandhill Dunnart}

Australia

Family THYLACINIDAE

Thylacinus cynocephalus (Harris 1808)

Thylacine, Tasmanian Wolf

Australia: Tasmania (ex)

\section{Family MYRMECOBIIDAE}

Myrmecobius fasciatus Waterhouse 1836

$$
\text { E } 1044,1098
$$

\section{Australia}




\section{Family PERAMELIDAE}

Chaeropus ecaudatus (Ogilby 1838)

Pig-footed Bandicoot

Australia (ex)

Echymipera clara Stein 1932

R

1098

Clara Bandicoot, White-lipped Bandicoot

Indonesia: Irian Jaya

Perameles bougainville Quoy \& Gaimard 1824

R

Western Barred Bandicoot, Marl, Barred Bandicoot

1

Australia: Bernier Island, Dorre Island

Perameles eremiana Spencer 1897

Ex 1044,1098

Desert Bandicoot

Australia (ex)

\section{Family THYLACOMYIDAE}

Macrotis lagotis (Reid 1837)

I

E

$838,1044,1098$

Greater Bilby, Dalgyte, Greater Rabbit-eared Bandicoot

Australia

Macrotis leucura (Thomas 1887)

Ex

$838,1044,1098$

Lesser Bilby, Yallara, Lesser Rabbit-eared Bandicoot

Australia (ex)

\section{Family PHALANGERIDAE}

Phalanger lullulae Thomas 1896

E $\quad 1098$

(=P. orientalis lullulae)

Woodlark Island Cuscus

Papua New Guinea: Woodlark lsland

Phalanger maculatus (E. Geoffroy 1803)

$11 \quad-\quad 1044$

(= Spilocuscus maculatus)

Common Spotted Cuscus, Spotted Phalanger

Australia: Queensland [1181]; Indonesia: Ambon, Aru, Irian Jaya, Kei, Salaya lsland, Seram; Papua New Guinea

Phalanger orientalis (Pallas 1766)

$11 \quad-\quad 1044$

Grey Cuscus, Common Phalanger

Australia: Queensland [1181]; Indonesia: Irian Jaya, Moluccas; Papua New Guinea: Bismarck Archipelago; Solomon Islands

Papua New Guinea

Phalanger rufoniger Zimara 1937

R $\quad 1098$

(=P. atrimaculatus, Spilocuscus rufoniger)

Black-spotted Cuscus

Indonesia: Irian Jaya; Papua New Guinea

Phalanger vestitus (Milne-Edwards 1877)

$\mathbf{R}$

Silky Cuscus

Indonesia: Irian Jaya; Papua New Guinea 
Family PETAURIDAE

Gymnobelideus leadbeateri McCoy 1867

Leadbeater's Possum

Australia

Family BURRAMYIDAE

Burramys parvus Broom 1896

Mountain Pygmy-possum

Australia

Family VOMBATIDAE

Lasiorhinus kreffiii (Owen 1873)

1

E

$405,838,1044,1098$

(includes $L$. barnardi)

Northern Hairy-nosed Wombat, Queensland Hairy-nosed Wombat Australia

\section{Family MACROPODIDAE}

Bettongia gaimardi (Desmarest 1822)

Tasmanian Bettong, Eastern Bettong

Australia

Bettongia lesueuri (Quoy \& Gaimard 1824)

I

$\mathbf{R}$

$838,1044,1098$

Burrowing Bettong, Boodie, Lesueur's Rat-kangaroo

Australia: Bernier Island, Dorre Island, Boodie, Barrow Island

Bettongia penicillata Gray 1837

(includes $\boldsymbol{B}$. tropica)

Brush-tailed Bettong, Woylie

Australia

Caloprymnus campestris (Gould 1843)

1

ld

$838,1044,1098$

Desert Rat-kangaroo

Australia

Dendrolagus bennettianus De Vis 1887

Bennett's Tree-kangaroo

Australia

Dendrolagus dorianus notatus Matschie 1916

$-\quad$ V 1098

Highland Unicolored Tree-kangaroo

Indonesia: Irian Jaya; Papua New Guinea

Dendrolagus goodfellowi shawmayeri Rothschild \& Dollman 1836

( $=$ D. matschiei shawmayeri)

Shawmayer's Ornate Tree-kangaroo

Papua New Guinea

Dendrolagus inustus Müller 1840

li

Grizzled Tree-kangaroo

Indonesia: Irian Jaya, Mysool, ?West Schouten 1slands; Papua New Guinea [643] 
Australia

Australia (ex)

Lagorchestes hirsutus Gould 1844

Rufous Hare-wallaby, Western Hare-wallaby

Australia

Lagorchestes leporides Gould 1841

Eastern Hare-wallaby

Australia (ex)

Lagostrophus fasciatus (Peron \& Lesueur 1807)

Banded Hare-wallaby

Ex 1044,1098

Australia: Bernier, Dorre lsland, Dirk Hartog Island

Macropus greyi Waterhouse 1846

Toolache Wallaby

Ex 1044,1098

Australia (ex)

Onychogalea fraenata (Gould 1841)

Bridled Nailtail Wallaby, Merrin

Australia

Onychogalea lunata (Gould 1841)

R $\quad 838,1044,1098$

Crescent Nailtail Wallaby, Wurrung

Australia (ex)

Petrogale persephone Maynes 1982

Prosperine Rock-wallaby

Australia

Potorous longipes Seebeck \& Johnston 1980 


\section{Order INSECTIVORA}

\section{Family SOLENODONTIDAE}

Solenodon cubanus Peters 1861

E $\quad 442,1098,1119,1120$

Cuban Solenodon, Almiqui

Cuba $[11,28]$

Solenodon paradoxus Brandt 1833

Haitian Solenodon, Hispaniolan Solenodon

Dominican Republic [1119]; Haiti [15,30]

\section{Family TENRECIDAE}

Geogale aurita aurita Milne-Edwards \& Grandidier 1872

Large-eared Tenrec

Madagascar

Geogale aurita orientalis Grandidier \& Petit 1930

Eastern Large-eared Tenrec

Madagascar

Limnogale mergulus Major 1896

Id $\quad 562,808$

Aquatic Tenrec, Web-footed Tenrec

Madagascar

Microgale brevicaudata G. Grandidier 1899

K $\quad 562,808$

Short-tailed Shrew-tenrec

Madagascar

Microgale crassipes Milne-Edwards 1893

K $\quad 562,808$

Large-footed Shrew-tenrec

Madagascar

Microgale drouhardi G. Grandidier 1934

K $\quad 562,808$

Drouhard's Shrew-tenrec

Madagascar

Microgale gracilis Mayer, 1896

K $\quad 808$

Gracile Shrew-tenrec

Madagascar

Microgale longicaudata Thomas 1883

K $\quad 562,808$

Lesser Long-tailed Shrew-tenrec

Madagascar

Microgale longirostris Major 1896

Long-nosed Shrew-tenrec

Madagascar

Microgale majori Thomas 1918

Major's Lesser Long-tailed Shrew-tenrec

Madagascar

Microgale melanorrhachis Morrison-Scott 1948

K $\quad 808$

Striped Shrew-tenrec

Madagascar 
Microgale occidentalis (G. Grandidier \& Petit 1931)

ld $\quad 562,808$

Western Short-tailed Shrew-tenrec

Madagascar

Microgale parvula G. Grandidier 1934

K $\quad 562,808$

Pygmy Shrew-tenrec

Madagascar

Microgale principula Thomas 1926

Greater Long-tailed Shrew-tenrec

K $\quad 562,808$

Madagascar

Microgale prolixicaudata G. Grandidier 1937

Id $\quad 562,808$

Northern Lesser Long-tailed Shrew-tenrec

Madagascar

Microgale pulla Jenkins 1988

K $\quad 564,808$

Dusky Shrew-tenrec, Dark Pygmy Shrew-tenrec

Madagascar

Microgale sorella Thomas 1926

K $\quad 562,808$

Long-tailed Shrew-tenrec

Madagascar

Microgale taiva Major 1896

K $\quad 562,808$

Taivi Shrew-tenrec

Madagascar

Microgale thomasi Major 1896

K $\quad 562,808$

Thomas's Shrew-tenrec

Madagascar

Family POTAMOGALIDAE

Micropotamogale lamottei Heim de Balsac 1954

E $\quad 808$

Nimba Otter-shrew

Côte d'Ivoire; Guinea; Liberia

Micropotamogale ruwenzorii (de Witte \& Frechkop 1955)

Ruwenzori Otter-shrew

1d $\quad 724$

Uganda [808]; Zaire [808]

Family CHRYSOCHLORIDAE

Amblysomus gunningi (Broom 1908)

Id

808,1014

Gunning's Golden Mole

South Africa

Amblysomus iris Thomas \& Schwann 1905

Id 808,1014

Zulu Golden Mole

South Africa

Amblysomus julianae Meester 1972

Juliana's Golden Mole

South Africa

Id

$27,724,725,1020$ 
Calcochloris obtusirostris (Peters 1851)

R $\quad 1014$

(= Chlorotalpa obtusirostris)

Yellow Golden Mole

Mozambique [808]; South Africa [808]; Zimbabwe [808]

Chlorotalpa duthiae (Broom 1907)

Duthie's Golden Mole

South Africa

Chlorotalpa sclateri (Broom 1907)

Id 808,1014

Sclater's Golden Mole

South Africa

Chlorotalpa tytonis (Simonetta 1968)

Somali Golden Mole

Somalia

Chrysochloris visagiei Broom 1950

Id 808,1014

Visagie's Golden Mole

South Africa

Chrysospalax trevelyani (Gūnther 1875)

R

$27,724,725,874,875,1020$

Giant Golden Mole

South Africa

Chrysospalax villosus (A. Smith 1833)

Rough-haired Golden Mole

R $\quad 808,1014$

South Africa

Cryptochloris wintoni (Broom 1907)

Id 808,1014

De Winton's Golden Mole

South Africa

Cryptochloris zyli Shortridge \& Carter 1938

Van Zyl's Golden Mole

South Africa

Eremitalpa granti (Broom 1907)

R

1014

Grants' Golden Mole

Namibia [808]; South Africa [808]

Family ERINACEIDAE

Erinaceus frontalis A. Smith 1831

R $\quad 724$

(= Atelerix frontalis)

South African Hedgehog

Angola [499]; Botswana [1020]; Lesotho [1020]; Namibia [1020]; South Africa [725, 1020]; Zimbabwe [1020]

Podogymnura truei Mearns 1905

V $\quad 27$

Mindanao Gymnure, Mindanao Moonrat

Philippines: Mindanao

Family SORICIDAE

Crocidura ansellorum Hutterer \& Dippenaar 1987

K

537,538

Zambia 
Ethiopia

Crocidura congobelgica Hollister 1916

Zaire

Crocidura crenata Brosset, DuBost \& Heim de Balsac 1965

Gabon

Crocidura dolichura ludia Hollister 1916

Zaire

Crocidura dolichura polia Hollister 1916

(=C. polia)

Zaire

Crocidura eisentrauti Heim de Balsac 1957

Cameroon

Crocidura glassi Heim de Balsac 1966

Ethiopia

Crocidura grassei Brosset, DuBost \& Heim de Balsac 1965

Cameroon; Central African Republic; Gabon

Crocidura kivuana Heim de Balsac 1968

K $\quad 808$

Zaire

Crocidura lanosa Heim de Balsac 1968

Rwanda; Zaire

Crocidura latona Hollister 1916

Zaire

Crocidura longipes Hutteser \& Happold 1983

K $\quad 539,808$

Nigeria

Crocidura lucina Dippenaar 1980

Ethiopia

K $\quad 285,808$

Crocidura luna raineyi Heller 1912

(= C. raineyi)

Kenya

Crocidura luna selina Dollman 1915

(= C. selina)

Uganda

Crocidura manengubae Hutterer 1981

Cameroon 
Crocidura monax Thomas 1910

K $\quad 808$

Kenya; Tanzania; Zaire

Crocidura nimbae Heim de Balsac 1956

K $\quad 808$

Côte d'Ivoire; Guinea; Liberia

Crocidura odorata goliath Thomas 1906

Ex $\quad 724$

Cameroon (ex)

Crocidura phaeura Osgood 1936

Ethiopia

Crocidura poensis thomensis Bocage 1887

K

(=C. thomensis)

São Tomé

Crocidura stenocephala Dieterlen \& Heim de Balsac 1979

(=C. littoralis stenocephla)

Zaire [282]

Crocidura tansaniana Hutterer 1986

Tanzania

Crocidura telfordi Hutterer 1986

Tanzania

Crocidura thalia Dippenaar 1980

Ethiopia

K 285,808

Crocidura usambarae Dippenaar 1980

K $\quad 285$

Tanzania

Crocidura wimmeri Heim de Balsac \& Aellen 1958

K $\quad 808$

Cameroon; Côte d'lvoire; Gabon; Liberia

Crocidura zimmermanni Wettstein 1953

R

Greece: Crete

Myosorex eisentrauti Heim de Balsac 1968

K $\quad 808$

Cameroon; Equatorial Guinea

Myosorex geata (Allen \& Loveridge 1927)

K $\quad 724,808$

Tanzania

Myosorex longicaudalus Meester \& Dippenaar 1978

K $\quad 808$

Long-tailed Forest Shrew

South Africa

Myosorex polli Heim de Balsac \& Lamotte 1956

K $\quad 724,808$

Zaire

Myosorex schalleri Heim de Balsac 1967

Zaire

K $\quad 724,808$

Paracrocidura graueri Hutterer 1986

K $\quad 536$

Zaire 
Paracrocidura maxima Heim de Balsac 1959

K $\quad 80$

Rwanda; Uganda; Zaire

Suncus remyi Brosset, DuBost \& Heim de Balsac 1965

K $\quad 724$

Gabon Dwarf Shrew

Gabon

Sylvisorex howelli Jenkins 1984

K $\quad 563,808$

Tanzania

Sylvisorex olulla Thomas 1913

K $\quad 808$

Cameroon; Gabon; Zaire

Sylvisorex suncoides Osgood 1936

Id $\quad 535,808$

(= Ruwenzorisorex suncoides)

?Rwanda; Uganda; Zaire

Family TALPIDAE

Desmana moschata (Linnaeus 1758)

V $\quad 27,106$

Russian Desman

Belarus [106]; Estonia [106]; Kazakhstan [106]; Latvia [106]; Lithuania [106]; Russia [106]; Turkmenia [106];

Ukraine [106,808]

Galemys pyrenaicus (E. Geoffroy 1811)

V

$27,811,841,876,1042$

Pyrenean Desman

?Andorra; France; Portugal; Spain

\section{Order MACROSCELIDEA}

Family MACROSCELIDIDAE

Petrodromus tetradactylus sangi (Heller 1912) $\quad-\quad$ K $\quad 240,808$

Four-toed Elephant-shrew

Kenya

Rhynchocyon chrysopygus Gūnther 1881

V $\quad 808$

Golden-rumped Elephant-shrew, Yellow-rumped Elephant-shrew

Kenya

Rhynchocyon cirnei cirnei Peters 1847

K $\quad 808$

Chequered Elephant-shrew

Mozambique

Rhynchocyon cirnei hendersoni Thomas 1902

R $\quad 78,808$

Henderson's Chequered Elephant-shrew

Malawi

Rhynchocyon petersi Bocage 1880

R $\quad 808$

Black-and-rufous Elephant-shrew

Kenya [808]; Tanzania: including Pemba Island, Zanzibar [808] 


\section{Order CHIROPTERA}

\section{Family PTEROPODIDAE}

Acerodon celebensis (Peters 1867)

(includes Pteropus arquatus Miller \& Hollister 1921)

Sulawesi Flying-fox

Indonesia: Saleyer, Sula Mangoli, Sulawesi

Acerodon humilis K. Andersen 1909

Talaud Flying-fox

Indonesia: Talaud Islands

Acerodon jubatus (Eschscholtz 1831)

Philippines

Acerodon lucifer Elliot 1896

II

Ex

Panay Giant Fruit Bat

Philippines: Panay (ex)

Acerodon mackloti (Temminck 1837)

Sunda Fying-fox

Indonesia: Alor, Flores, Lombok, Sumba, Sumbawa, Timor

Alionycteris paucidentata Kock 1969

K

Philippines: Mindanao

Aproteles bulmerae Menzies 1977

K

Bulmer's Fruit Bat

Papua New Guinea: Western Province

Dobsonia exoleta chapmani Rabor 1952

Ex?

$483,742,913$

(=D. chapmani)

Chapman's Fruit Bat

Philippines: Cebu, Negros (ex?)

Dobsonia minor (Dobson 1879)

K

Lesser Naked-backed Fruit Bat

Indonesia: Irian Jaya [352,913], Sulawesi [145]; Papua New Guinea [352]

Dyacopterus spadiceus (Thomas 1890)

$\mathbf{K}$

(except subspecies brooki)

Dayak Fruit Bat

Malaysia: Peninsular Malaysia, Sabah, Sarawak; Philippines: ?Mindanao

Dyacopterus spadiceus brooki Thomas 1920

(= D. brooki)

Indonesia: Sumatra [913]; Philippines: Luzon

Latidens salimalii Thonglongya 1972

India 
Nyctimene rabori Heaney \& Peterson 1984

E

483,742

Philippines Tube-nosed Fruit Bat

Philippines: Negros

Otopteropus cartilagonodus Kock 1969

Philippines: Luzon

483,742

Pteropus admiralitatum Thomas 1894

II

742

Admiralty Flying-fox

Papua New Guinea: Admiralty Islands, Bougainville, New Britain, Tabar Islands; Solomon Islands

Pteropus alecto Temminck 1837

II

742

Central Flying-fox

Australia; Indonesia: Bawean Island, Kangean Islands, Salaya Island, Sawu, Sulawesi, Sumba; Papua New Guinea

Pteropus anetianus Gray 1870

II

Vanuatu

Pteropus argentatus
Silvery Flying-fox

Indonesia: Ambon

Pteropus balutus Hollister 1913

II

(= P. pumilus balutus)

Philippines: Saragani Island

Pteropus brunneus Dobson 1878

II

Australia: Percy Island

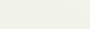

Pteropus caniceps Gray 1870

Ashy-headed Flying-fox

Indonesia: Bacan, Halmahera, Sangihe, Sula Islands, Sulawesi, Ternate

Pteropus chrysoproctus Temminck 1837

11

Ambon Flying-fox

Indonesia: Ambon, Buru, Gorong Islands, Keffing, Sangihe, Seram, Watebula Islands

Pteropus conspicillatus Gould 1849

II

Spectacled Flying-fox

Australia; Indonesia: Halmahera, Irian Jaya; Papua New Guinea

Pteropus dasymallus Temminck 1825

II

742

Ryukyu Flying-fox

Japan: Kyushu, Ryukyu Islands; Taiwan

Pteropus faunulus Miller 1902

India: Nicobar Islands

11

Pteropus fundatus Felten \& Kock 1972

II

Vanuatu: Banks Island

Pteropus giganteus (Brünnich 1782)

II $\quad$ - $\quad 742$

Indian Flying-fox

Bangladesh; China; India: including Andaman Islands; Maldives; Nepal; Pakistan; Sri Lanka 
Papua New Guinea: New Britain

$\begin{array}{llll}\text { Pteropus griseus (E. Geoffroy 1810) } & 11 & - & 742\end{array}$

Grey Flying-fox

Indonesia; Philippines: ?Luzon

Pteropus howensis Troughton 1931

Small Flying-fox

Australia; Cambodia; Indonesia; Malaysia: islands off Sabah; Maldives; Myanmar; Papua New Guinea; Philippines; Solomon Islands; Thailand; Viet Nam

Pteropus insularis Hombron \& Jacquinot 1842

Truk Flying-fox

Federated States of Micronesia: Truk

Pteropus leucopterus Temminck 1853

Philippines: Luzon

Pteropus leucotis Sanborn 1950

Philippines: Busuanga, Palawan

Pteropus livingstonei Gray 1866

Comoro Black Flying-fox

Comoros: Anjouan, Moheli

Pteropus lombocensis Dodson 1878

Lombok Flying-fox

Indonesia: Alor, Flores, Lombok

Pteropus lylei K. Andersen 1908

\section{Lyle's Flying-fox}

Cambodia; Thailand; Viet Nam

Pteropus macrotis Peters 1867

Big-eared Flying-fox

Indonesia: Aru Islands, Irian Jaya; Papua New Guinea

Pteropus mahaganus Sanborn 1931

Lesser Flying-fox

Papua New Guinea: Bougainville; Solomon Islands: Santa 1sabel

Pteropus mariannus Desmarest 1822

Micronesian Flying-fox

Federated States of Micronesia: Kosrae, Ulithi Atoll, Yap; Guam; Japan: Okinawa; Northern Marianas: Aguijan, Alamagan, Pagan, Saipan, Tinian; Palau 
Christmas Island; India: Andaman and Nicobar Islands; Indonesia: Enggano, Nias

Pteropus molossinus Temminck 1853

Pohnpei Flying-fox

Federated States of Micronesia: Mortlock Island, Pohnpei

Pleropus neohibernicus Peters 1876

Bismarck Flying-fox

Indonesia: Irian Jaya; Papua New Guinea

Pteropus niger (Kerr 1792)

Greater Mascarene Flying-fox

Mauritius; Reunion (ex)

Pteropus nitendiensis Sanborn 1930

Solomon Islands: Ndeni Island

Pteropus ocularis Peters 1867

Seram Flying-fox

Indonesia: Buru, Seram

Pteropus ornatus Gray 1870

New Caledonia

Pteropus personatus Temminck 1825

\section{Masked Flying-fox}

Indonesia: Halmahera, ?Sulawesi, Ternate

Pteropus phaeocephalus Thomas 1882

Mortlock Flying-fox

Federated States of Micronesia: Chuuk, Mortlock Island

Pteropus pilosus K. Andersen 1908

Large Palau Flying-fox

Palau (ex)

Pteropus pohlei Stein 1933

( = P. macrotis pohlei)

Indonesia: Yapen

Pteropus poliocephalus Temminck 1825

Grey-headed Flying-fox

Australia; Indonesia: Timor

Pteropus pselaphon Lay 1829

Japan: Bonin Islands, Volcano Islands 
Solomon Flying-fox

Solomon Islands

Pteropus rodricensis Dobson 1878

11

Rodrigues Flying-fox

Mauritius: Rodrigues, Round Island (ex)

Pteropus rufus E. Geoffroy 1803

I1

Madagascar Flying-fox

Madagascar

Pteropus samoensis Peale 1848

I E

Samoan Flying-fox

American Samoa; Fiji; Samoa

Pteropus santacrucis Troughton 1930

(= P. hypomelanus santacrucis)

Solomon Islands: Santa Cruz Islands

Pteropus scapulatus Peters 1862

Little Red Flying-fox

Australia; New Zealand; Papua New Guinea

Pteropus seychellensis Milne-Edwards 1877

(except subspecies aldabrabensis)

Seychelles Flying-fox

Comoros; Seychelles; Tanzania: Mafia Island

Pteropus seychellensis aldabrensis True 1893

11

V

Aldabra Flying-fox

Seychelles: Aldabra Island

Pteropus speciosus K. Andersen 1908

Indonesia: Mata Siri, Salembu Besar; Philippines

Pteropus subniger (Kerr 1792)

II

Ex

Lesser Mascarene Flying-fox

Mauritius (ex); Reunion (ex)

Pteropus tablasi Taylor 1934

(=P. pumilus tablasi)

Philippines

Pteropus temmincki Peters 1867

Temminck's Flying-fox

Indonesia: Ambon, Buru, Seram, ?Timor; Papua New Guinea: Bismarck Archipelago

Pteropus tokudae Tate 1934

II

Ex

$27,174,742,849,974$

Guam Flying-fox

Guam (ex)

Pteropus tonganus Quoy \& Gaimard 1830

Insular Flying Fox

American Samoa; Cook Islands; Fiji; New Caledonia; Niue; Papua New Guinea; Samoa; Solomon Islands: Karkar, Rennell; Tonga; Vanuatu 
Large Flying-fox

Brunei; Cambodia; Indonesia; Malaysia: Peninsular Malaysia, Sabah, Sarawak; Myanmar; Philippines; Thailand; Tonga; Vanuatu

Pteropus vetulus Jouan 1863

New Caledonia

Pteropus voeltzkowi Matschie 1909

II

E

Pemba Flying-fox

Tanzania: Pemba Island

Pteropus woodfordi Thomas 1888

II

Least Flying-fox

Solomon Islands

\section{Family EMBALLONURIDAE}

Coleura seychellensis Peters 1869

Seychelles Sheath-tailed Bat

Seychelles

Emballonura furax Thomas 1911

Greater Sheath-tailed Bat

Indonesia: Irian Jaya [1118]; Papua New Guinea [352]

Emballonura raffrayana Dobson 1879

K

Raffray's Sheath-tailed Bat

Indonesia: Irian Jaya, Kai Islands, Seram, Sulawesi [352,1118]; Papua New Guinea [352]; Solomon Islands

\section{Family CRASEONYCTERIDAE}

Craseonycteris thonglongyai Hill 1974

$\mathbf{R}$

Kitti's Hog-nosed Bat

Thailand [500]

Family MEGADERMATIDAE

Macroderma gigas (Dodson 1880)

V $527,1044,1098$

Ghost Bat, Australian False Vampire

Australia

\section{Family RHINOLOPHIDAE}

Hipposideros papua (Thomas \& Doria 1886)

Geelvinck Bay Leaf-nosed Bat

Indonesia: Biak, Irian Jaya, northem Moluccas 
Rhinonicteris aurantius (Gray 1845)

K

1143

Orange Leaf-nosed Bat, Orange Horseshoe Bat

Australia

\section{Family PHYLLOSTOMIDAE}

Leptonycteris nivalis (Saussure 1860)

V $80,243,488$

Big Long-nosed Bat, Mexican Long-nosed Bat

Guatemala; Mexico [906]; USA

Leptonycteris yerbabuenae Martinez \& Villa-Ramirez 1940

(= L. sanborni Hoffmeister 1957)

Little Long-nosed Bat

V $\quad 80$

El Salvador; Guatemala; Mexico [906]; USA

Phyllonycteris aphylla (Miller 1898)

K $\quad 717$

Jamaican Flower Bat

Jamaica

Phyllonycteris major Anthony 1917

Puerto Rican Flower Bat

Puerto Rjco (ex)

Phyllonycteris poeyi Gundlach 1861

Cuban Flower Bat

Cuba; Dominican Republic; Haiti

Vampyrops lineatus (E. Geoffroy 1810) $111 \mathrm{UY}-\quad 625$

White-lined Bat

Argentina [586,915]; Bolivia; Brazil; Colombia [316]; Paraguay [915]; Peru; Uruguay [400,401,915]

Family MYZOPODIDAE

Myzopoda aurita Milne-Edwards \& Grandidier 1878

Sucker-footed Bat

Madagascar

Family VESPERTILIONIDAE

Glischropus javanus Chasen 1939

K $\quad 1118$

Javan Thick-thumbed Bat

Indonesia: Java

Kerivoula africana Dobson 1878

Ex? 724

Tanzanian Woolly Bat

Tanzania (ex?)

Lasiurus cinereus semotus (Peale \& Beauvois 1796)

Id

1098

Hawaiian Hoary Bat

Hawajian Islands

Myotis capaccinii (Bonaparte 1837)

V $138,179,243,267,313,514,630$, 1040

\section{Long-fingered Bat}

Algeria; Austria; Bulgaria [160]; France [340]; Greece; Iraq [466,687]; Iran [646]; 1srael [466]; Italy; Jordan [466]; Lebanon [466]; Malta; Morocco; Spain: including Balearic Islands [406]; Turkey [466,629]; Uzbekistan; Yugoslavia 
Myotis dasycneme (Boie 1825)

K

$243,313,514,1040$

Pond Bat

Austria; Belarus; Belgium; China; Czechoslovakia; Denmark [138]; Estonia; France [138,340]; Germany; Hungary; Kazakhstan; Latvia [1092]; Lithuania; Luxembourg; Netherlands [138,254]; Poland; Russia; Sweden [138]; Switzerland; Ukraine [1068]

Myotis formosus bartelsi Jentink 1910

K

1118

(= M. bartelsi)

Bartel's Myotis

Indonesia: Java

Myot is formosus hermani Thomas 1923

R

913,1118

(= M. hermani)

Herman's Myotis, Herman's Bat

Indonesia: Sumatra

Myotis grisescens A.H. Howell 1909

E $\quad 442,1098$

Grey Myotis, Grey Bat

USA

Myotis myotis (Borkhausen 1797)

K

$179,238,243,406,514,1040$

Large Mouse-eared Bat

Albania; Austria; Azores; Belarus; Belgium; Bulgaria [313]; Cyprus; Czechoslovakia [190]; France [340]; Germany; Greece; Hungary; Israel [466]; Italy; Jordan; Lebanon [466,630]; Liechtenstein; Luxembourg; Malta [988]; Netherlands [254]; Poland; Portugal; Romania; Slovenia [842]; Spain [406]; Switzerland; Syria [466]; Turkey [629]; Ukraine; United Kingdom (ex?); Yugoslavia

Myotis sodalis Miller \& Allen 1928

V $442,1094,1098$

Indiana Bat, Social Bat

USA

Plecotus townsendii Cooper 1837

Id $\quad 95,632,1098$

Townsend's Big-eared Bat

Canada; Mexico [906]; USA

\section{Family MYSTACINIDAE}

Mystacina robusta Dwyer 1962

Ex

351

New Zealand Greater Short-tailed Bat

New Zealand: Big South Cape Island, Solomon Island (ex)

Mystacina tuberculata Gray 1843

V

258

New Zealand Lesser Short-tailed Bat

New Zealand

Family MOLOSSIDAE

Otomops formosus Chasen 1939

K

Javan Mastiff Bat

Indonesia: Java 


\section{Order PRIMATES}

\section{Family TUPAIIDAE}

(listed in a separate Order (SCANDENTIA) by Honacki et al. [514] and Corbet and Hill [243])

Anathana ellioti (Waterhouse 1850)

Madras Treeshrew

India

Dendrogale melanura (Thomas 1892)

Bornean Smooth-tailed Treeshrew

Malaysia: Sabah, Sarawak

Dendrogale murina (Schlegel \& Müller 1845)

Northern Smooth-tailed Treeshrew

Cambodia; ?Lao P.D.R. [655]; Thailand [151]; Viet Nam [198, 1025]

Prilocercus lowii Gray 1848

11

11

217,723

Pen-tailed Treeshrew

Brunei; Indonesia [565,934]; Malaysia: Peninsular Malaysia [721], Sabah, Sarawak; Thailand [151]

Tupaia dorsalis Schlegel 1857

11

723

Striped Treeshrew

Brunei; Indonesia: Kalimantan; Malaysia: Sabah, Sarawak

Tupaia glis (Diard 1820)

II

514

(includes $T$. belangeri)

Common Treeshrew

Bangladesh [602,604]; ?Bhutan; Brunei [119]; Cambodia [837]; China [1169]; India [763]; Indonesia: Java, Kalimantan [1009], Sumatra [934]; Lao P.D.R. [271,417,837]; Malaysia: Peninsular Malaysia, Sabah [266], Sarawak [217]; Myanmar; Nepal [573,756]; Singapore [469]; Thailand [151]; Viet Nam [261,837,1025]

Tupaia gracilis Thomas 1893

11

217,723

Slender Treeshrew

Indonesia: Bangka, Belitung, Kalimantan [1009], Karimata lslands; Malaysia: Sabah [266], Sarawaik

Tupaia javanica Horsfield 1822

11

217

Javan Treeshrew

Indonesia: Bali, Java, Nias, Sumatra [934]

Tupaia minor Gūnther 1876

II

217,723

Pygmy Treeshrew, Lesser Treeshrew

Brunei [119]; Indonesia: Kalimantan, Lingga Archipelago, Sumatra [934]; Malaysia: Peninsular Malaysia, Sabah [266], Sarawak; Thailand [151]

Tupaia montana Thomas 1892

11

217,723

Mountain Treeshrew

Indonesia: Kalimantan [1009]; Malaysia: Sabah [266], Sarawak

Tupaia nicobarica (Zelebor 1869)

Nicobar Treeshrew

India: Great and Little Nicobar Islands

Tupaia palawanensis Thomas 1894

11

Palawan Treeshrew

Philippines: Busuanga, Culion, Cuyo, Palawan 
Ruddy Treeshrew

Indonesia: Bunguran, Kalimantan [1009]

Tupaia tana Raffles 1821

Large Treeshrew

Brunei; Indonesia [934]; Malaysia: Sabah [266], Sarawak

Urogale everetti (Thomas 1892)

Philippine Treeshrew

Philippines: Mindanao

Family LEMURIDAE (including MEGALADAPIDAE)

Hapalemur aureus Meier, Albignac, Peyrieras, Rumpler \& Wright 1987

Madagascar

Golden Bamboo Lemur, Golden Lemur

Hapalemur griseus alaotrensis Rumpler \& Albignac 1975

Alaotran Gentle Lemur

Madagascar

Hapalemur griseus griseus (Link 1795)

Grey Gentle Lemur

Madagascar

Hapalemur griseus occidentalis Rumpler \& Albignac 1975

Western Gentle Lemur

Madagascar

Hapalemur simus Gray 1870

Broad-nosed Gentle Lemur, Greater Bamboo Lemur Madagascar

Lemur catta Linnaeus 1758

\section{Ring-tailed Lemur}

Madagascar [724]

Lemur coronatus Gray 1842

(= L. mongoz coronatus, Petterus coronatus)

\section{Crowned Lemur}

Madagascar [724]

Lemur fulvus albifrons

E. Geoffroy 1796

1

White-fronted Lemur

Madagascar
$175,577,717,860,1037,1060$, $1061,1062,1077,1183$

V

E 1188

$816,860,881,881,901,1037$. 1077

$27,397,724,1037,1077,1183$,

\section{$724,860,1037,1077,1183$}

$577,724,816,860,914,1017$, $1037,1077,1078$ 
$577,816,860,914,1037,1077$, 1078

\section{White-collared Lemur}

Madagascar

Lemur fulvus collaris E. Geoffroy 1812

Collared Lemur

Madagascar

Lemur fulvus fulvus $\quad$ E. Geoffroy 1796

R $461,577,724,816,860,914,1037$, 1077

\section{Brown Lemur}

Madagascar

Lemur fulvus mayottensis Schlegel 1886

Mayotte Lemur

Mayotte

Lemur fulvus rufus (Audebert 1799)

(=L. macaco rufus)

Red-fronted Lemur

Madagascar

Lemur fulvus sanfordi Archbold 1932

(= L. macaco sanfordi)

\section{Sanford's Lemur}

Madagascar

Lemur macaco flavifrons (Gray 1867)

Sclater's Lemur

Madagascar [724]

Lemur macaco macaco Linnaeus 1766

\section{Black Lemur}

Madagascar [724]

Lemur mongoz Linnaeus 1766

$\mathbf{R}$

$27,577,724,860,901,1037,1059$

\section{Mongoose Lemur}

Comoros: Moili and Nodzoavani Islands; Madagascar

Lemur rubriventer I. Geoffroy 1850

\section{Red-bellied Lemur}

Madagascar

Lepilemur dorsalis Gray 1870

E

$27,577,724,860,1037,1077$, 1183

(= L. mustelinus dorsalis)

Grey-backed Sportive Lemur, Nossi-be Sportive Lemur Madagascar

Lepilemur edwardsi Forbes 1894 


\section{Ruffed Lemur}

Madagascar

Family CHEIROGALEIDAE

Allocebus trichotis (Günther 1875)

(= Cheirogaleus trichotis)

Hairy-eared Dwarf Lemur

Madagascar

Cheirogaleus major E. Geoffroy 1812

Greater Dwarf Lemur

Madagascar

Cheirogaleus medius E. Geoffroy 1812

$27,577,724,816,860,901,1037$, $1061,1062,1077,1183$

Fat-tailed Dwarf Lemur

Madagascar

Microcebus coquereli A. Grandidier 1867

( $=$ Mirza coquereli)

Coquerel's Mouse-lemur, Coquerel's Dwarf Lemur

Madagascar

Microcebus murinus (J.F. Miller 1777) 
Microcebus rufus E. Geoffroy 1834

(= M. murinus rufus)

Brown Mouse-lemur, Rufous Mouse-lemur, Russet Mouse-lemur

Madagascar

Phaner furcifer (Blainville 1841)

Fork-marked Lemur, Fork-marked Mouse-lemur

Madagascar

Family INDRIIDAE

Indri indri (Gmelin 1788)

Indris

Madagascar [724]

Lichanotus laniger (Gmelin 1788)

(= Avahi laniger)

Woolly Lemur

Madagascar

Propithecus diadema Bennett 1832

Diademed Sifaka

Madagascar [724]

Propithecus tattersalli Simons 1988

Golden-crowned Sifaka, Tattersall's Sifaka

Madagascar

Propithecus verreauxi A. Grandidier 1867

\section{Verreaux's Sifaka}

Madagascar

\section{Family DAUBENTONIIDAE}

Daubentonia madagascariensis (Gmelin 1788)

\section{Aye-aye}

Madagascar

Family LORISIDAE

Arctocebus calabarensis (J.A. Smith 1860)

Golden Potto, Angwantibo

Angola [518,531]; Cameroon [691]; ?Central African Republic [759,823]; Congo [691]; Equatorial Guinea [578]; Gabon [141,214]; Nigeria [724,819]; ?Zaire [759,823,991]

Loris sardigradus (Linnaeus 1758)

11

1183
R $\quad 27,577,816,860,901,1037,1061$, $1062,1077,1183$

$27,914,1037,1077,1183$

$27,577,724,816,860,901,1037$, $1061,1062,1077,1079,1183$

$27,371,724,816,860,1037,1077$, 1183

II $\quad \mathrm{K} \quad 443,649,724,1183$
$816,914,1037,1077$

Slender Loris

India $[38,969,1034]$; Sri Lanka $[318,861,865]$ 
Nycticebus coucang (Boddaert 1785)

11

$649,723,1183$

Slow Loris

Bangladesh [393,453,601,602,603,604,605]; Brunei; Cambodia; China [1072, 1202]; India [38,763]; Indonesia: Bangka, Java, Kalimantan [1009], Natuna Islands, Rhio Archipelago [934], Sumatra; Lao P.D.R. [271,837]; Malaysia: Peninsular Malaysia [707], Sabah, Sarawak; Myanmar [1109]; Philippines: Sulu Archipelago [892]; Singapore (ex?); Thailand [151,216]; Viet Nam [684,837,853,1025]

$\begin{array}{lllll}\text { Nycticebus pygmaeus Bonhote } 1907 & \text { II } & \text { V } & 1183\end{array}$

Pygmy Loris, Lesser Slow Loris

?Cambodia [514]; Lao P.D.R. [422,837,976]; Viet Nam $[684,837,853,1025]$

Perodicticus potto (P.L.S. Müller 1766) $\quad 11 \quad$ - $\quad 435,443,724,1183$

Potto, Potto Gibbon

Angola [518,531]; Benin [978]; Burundi [1179]; Cameroon [759]; Central African Republic [862]; Congo [862]; Côte d'lvoire [162]; Equatorial Guinea [182]; Gabon [141,214,215]; Ghana [559]; Guinea [155]; Guinea-Bissau [155]; Kenya [611,897]; Liberia [14,628]; Nigeria [431,940]; Rwanda [322,895]; Sierra Leone [155,263]; Togo [155]; Uganda [120,611]; Zaire [233,759,896,991]

\section{Family GALAGIDAE}

Galago alleni Waterhouse 1838

I1

$443,724,1183$

Allen's Galago, Allen's Bushbaby

Angola [518]; Cameroon [557]; Central African Republic [759,823]; Congo [691]; Equatorial Guinea [182,1093]; Gabon [141,214]; Nigeria [940]

Galago demidovii (Fischer 1806)

11

$435,724,1183$

(= Galagoides demidoff)

(except subspecies thomasi)

Demidoff's Galago, Dwarf Bushbaby

Angola [480,518]; Benin [978]; Burkina Faso [944]; Cameroon [557]; Central African Republic [759,823]; Congo [691]; Côte d'lvoire [162]; Equatorial Guinea [182, 1093]; Gabon [141,214]; Ghana [1134]; Guinea [937, 1134]; Liberia [14,628]; Mali [1134]; Nigeria [940]; Rwanda [594]; Senegal (ex?) [305]; Sierra Leone [263,1086]; Tanzania [1067]; Togo [1134]; Zaire [233,896,991]

Galago demidovii thomasi (Elliott 1907)

11

K

649

(= Galago thomasi, Galagoides demidoff thomasi)

Thomas's Bushbaby

?Kenya; ?Malawi [1 134]; Uganda [120,611]; Zaire

Galago elegantulus Le Conte 1857

11

724,1183

(= Euoticus elegantulus)

Western Needle-clawed Galago, Western Needle-clawed Bushbaby

?Angola [518,823,862]; Cameroon[557]; Central African Republic [759,823]; Congo [611]; Equatorial Guinea [182,1093]; Gabon [141,214]; Nigeria [940]; ?Zaire [759,823]

Galago granti Thomas \& Wroughton 1907 724

(= G. senegalensis granti, Galagoides zanzibaricus granti)

Grant's Galago, Grant's Bushbaby

Malawi [78]; Mozambique [1017]; Zimbabwe [1020]

Galago inustus Schwarz 1930

11

$443,724,1183$

(= Euoticus inustus)

Eastern Needle-clawed Galago, Eastern Needle-clawed Bushbaby

Rwanda?[1135]; Uganda [61 1]; Zaire [895,991] 
(includes G. moholi A. Smith 1839)

Lesser Galago, Lesser Bushbaby, Senegal Galago

Angola [499,518]; Benin [978]; Botswana [1020]; Burkina Faso [944]; Cameroon [645]; Central African Republic [862, 1134]1; Chad [689]; Côte d'lvoire [155]; ?Djibouti [611]; Ethiopia [1192]; ?Gambia; Ghana [153]; Guinea [155]; Guinea-Bissau [155]; Kenya [611]; Liberia [862]; Malawi [78,452,1066]; Mali [155]; Mozambique [1017,1020]; Namibia [1020]; Niger [872]; Nigeria [940]; Rwanda [551]; Senegal [305,307]; Sierra Leone [1086]; Somalia [337,367]; South Africa [725,1020]; Sudan [996]; Swaziland [1020]; Tanzania [1067]; Togo [155]; Uganda [120,611]; Zaire [991]; Zambia [75]; Zimbabwe [1020]

Galago zanzibaricus Matschie 1893 1I V $\quad 649,1014$

(= G. senegalensis zanzibaricus)

Zanzibar Galago, Zanzibar Bushbaby

Kenya [611]; Malawi [452]; Mozambique [514]; Tanzania: including Zanzibar [611]

Otolemur crassicaudatus (E. Geoffroy 1812) II $\quad-\quad 443,724,1014,1018,1183$

(includes $O$. garnetti Ogilby 1838)

Thick-tailed Busbbaby, Greater Busbbaby

Angola [518]; Burundi; Kenya [611]; Malawi [78,452,1066]; Mozambique [1017,1620]; Rwanda [724]; Somalia [337,367]; South Africa [725,1020]; Swaziland [1020]; Tanzania: including Pemba 1sland, Zanzibar [771]; Uganda [120,611]; Zaire [991]; Zambia [75]; Zimbabwe [1020]

\section{Family TARSIIDAE}

Tarsius bancanus Horsfield 1821

II

$27,468,723,1183$

Western Tarsier

Brunei; Indonesia: Bangka, Belitung, Kalimantan [1009], Serasan [217], Sumatra [934]; Malaysia: Sabah [266], Sarawak [810]

Tarsius spectrum (Pallas 1779)

II

27,1183

(except subspecies pumilus)

Eastern Tarsier, Sulawesi Tarsier, Spectral Tarsier

Indonesia: Peleng, Salaya Island, Sangihe, Sulawesi

Tarsius spectrum pumilus Miller \& Hollister 1921

11

Id

27,782

(=T. pumilus)

Lesser Spectral Tarsier, Pygmy Tarsier

Indonesia: Sulawesi

Tarsius syrichta (Linnaeus 1758)

II

E

27,1183

Philippine Tarsier

Philippines: Bohol, Leyte, Mindanao, Samar

\section{Family CALLITHRICIDAE}

Callithrix argentata (Linnaeus 1766)

(except subspecies leucippe)

Silvery Marmoset, Black-tailed Marmoset

Bolivia [116,493]; Brazil [251,948]; Paraguay[915,1035]

Callithrix argentata leucippe (Thomas 1922)

11

V

788,1098

White Marmoset

Brazil 
(except subspecies chrysoleuca, intermedius)

Tassel-eared Marmoset

Brazil

Callithrix humeralifer chrysoleuca (Wagner 1842)

II

K

493

Brazil

Callithrix humeralifer intermedius (Hershkovitz 1977) I

Brazil

Callithrix jacchus (Linnaeus 1758)

(except subspecies aurita, flaviceps)

(includes C. geoffroyi (Humboldt 1812), C. penicillata (E. Geoffroy 18I2))

Common Marmoset

Bolivia [116]; Brazil

Callithrix jacchus aurita (E. Geoffroy 1812)

I $E$

$251,758,781,1098,1183$

$(=C$. aurita $)$

Buffy-tufted-ear Marmoset, White-eared Marmoset

Brazil

Callithrix jacchus flaviceps (Thomas 1903)

I

E

$184,251,478,758,788,1098$

(=C. flaviceps)

Buffy-headed Marmoset

Brazil

Cebuella pygmaea (Spix 1823)

II

$184,618,788,1021,1183$

Pygmy Marmoset

Bolivia [116,486]; Brazil [251,316,948]; Colombia [316,493]; Ecuador [493]; Peru [316, 1022,1023]

Leontopithecus rosalia caissara Lorini \& Persson $1990 \quad 1$

E $\quad 662$

(= L. caissara)

Black-faced Lion Tamarin

Brazil: Superagui Island

Leontopithecus rosalia chrysomelas (Kuhl 1820)

I

$E$

$184,251,692,758,788,1098$

(= L. chrysomelas)

Golden-headed Lion Tamarin

Brazil

Leontopithecus rosalia chrysopygus (Mikan 1820)

I

E

$184,251,692,758,788,1098$

(= L. chrysopygus)

Golden-rumped Lion Tamarin, Black Lion Tamarin

Brazil

Leontopithecus rosalia rosalia (Linnaeus 1766)

I

$E$

$184,251,618,692,758,788,1098$

(= L. rosalia)

Golden Lion Tamarin

Brazil

Saguinus bicolor (Spix 1823)

I

E

$92,184,251,315,618,788,948$, 1098

(includes S. martinsi (Thomas 1912))

Bare-faced Tamarin, Pied Tamarin

Brazil 
Saguinus fuscicollis (Spix 1823)

II

$618,788,1095,1183$

(includes S. tripartitus (Milne-Edwards 1878))

Saddle-back Tamarin, Brown-headed Tamarin, Rio Napo Tamarin

Bolivia [493]; Brazil [251,316,948]; Colombia [316,759]; Ecuador [493]; Peru [316,395, 1022, 1023]

Saguinus imperator (Goeldi 1907)

1I Id $184,618,788,1098$

Emperor Tamarin

Bolivia [493]; Brazil [948]; Peru [1022]

Saguinus inustus Schwarz 1951

11

$618,788,1183$

Mottle-faced Tamarin, Dusky Tamarin

Brazil [948]; Colombia [316,493]

Saguinus labiatus (E. Geoffroy 1812)

11

788,1183

Red-chested Tamarin, Red-bellied Tamarin, White-lipped Tamarin

Bolivia [1 16,184,618]; Brazil [251,948]; Colombia [490]; Peru [184,618,1022]

Saguinus leucopus (Günther 1877)

I

E $\quad 316,788,1098$

White-footed Tamarin

Colombia

Saguinus midas (Linnaeus 1758)

11

788,1183

(includes S. tamarin (Link 1795))

Red-handed Tamarin, Negro Tamarin

Brazil [184,251,618,948]; French Guiana [316,493]; Guyana [316,493]; Suriname [94,532]

Saguinus mystax (Spix 1823)

11

$184,618,788,1183$

(includes $S$. pileatus (I. Geoffroy \& Deville 1848))

Moustached Tamarin

Brazil [251,948]; Peru [395,905, 1022]

Saguinus nigricollis (Spix 1823)

II

$184,618,788,1183$

(includes S. graellsi (Jiménez de la Espada 1870))

Black-and-red Tamarin, Black-mantled Tamarin

Brazil [25 I,948]; Colombia [493]; Ecuador [493]; Peru [1022]

Saguinus oedipus (Linnaeus 1758)

$184,442,618,659,788$

(except subspecies oedipus)

(includes S. geoffroyi (Pucheran 1845))

Cotton-headed Tamarin, Geoffroy's Tamarin, Rufous-naped Tamarin

Colombia [316,493]; Costa Rica [493]; Panama [1012]

Saguinus oedipus oedipus (Linnaeus 1758)

Cotton-top Tamarin, Pinché

Colombia

\section{Family CALLIMICONIDAE}

Callimico goeldii (Thomas 1904)

$1 \quad \mathrm{R} \quad 845,1098,1183$

Goeldi's Marmoset, Goeldi's Monkey

Bolivia [116,493]; Brazil [251,948]; Colombia [316,493]; ?Ecuador; Peru [ i 022]

Family CEBIDAE

Alouarta belzebul (Linnaeus 1766)

Red-handed Howler, Black-and-red Howler

Brazil 
Alouatta caraya (Humboldt 1812)

I1

184,788

Black Howler

Argentina [124,699,701,915]; Bolivia [486]; Brazil [251]; Paraguay [915]

Alouatta fusca (E. Geoffroy 1812)

II Id

$184,788,1098,1183$

(includes A. beniensis Lōnnberg 1941)

(= A. guariba (Humboldt 1812))

Brown Howler

Argentina [124,915]; Bolivia [860]; Brazil [251,478,758]

$\begin{array}{lllll}\text { Alouatta palliata (Gray 1849) } & \text { I } & - & 442,788,1183\end{array}$

Mantled Howler

Colombia [316,490]; Costa Rica [11221; Ecuador; El Salvador; Guatemala; Honduras [703]; Mexico [906];

Nicaragua; Panama; Peru [202]

Alouatta pigra Lawrence 1933

11

K $\quad 442,522,788,1098$

(= A. villosa (Gray 1845 nomen dubium))

Guatemalan Howler

Belize [526]; Guatemala [404]; Mexico [906, 1150]
Alouatta seniculus (Linnaeus 1766)
11
$184,788,1183$

Red Howler

Bolivia [116,486]; Brazil [251,948]; Colombia [316,490]; Ecuador; French Guiana [316]; Guyana [316,776];

Peru [1022,1023]; Suriname [94,316,353,532,760]; Trinidad and Tobago [13]; Venezuela $[316,319,946]$

Aotus trivirgatus (Humboldt 1811)

II

$184,442,788,1183$

(includes A. azarae (Humboldt 1812), A. brumbacki, A. infulatus (Kuhl 1820), A. lemurinus I. Geoffroy 1843, A. miconax Thomas 1927, A. nancymai, A. nigriceps Dollman 1909, A. vociferans (Spix 1823))

Night Monkey, Douroucouli, Owl Monkey

Argentina [124,910,915]; Bolivia; Brazil [251,854,948]; Colombia [316,490]; Ecuador; Panama; Paraguay [915]; Peru [1022,1023]; Venezuela [316,946]

$\begin{array}{lllll}\text { Ateles belzebuth E. Geoffroy } 1806 & \text { II } & \text { V } & 184,624,788,1098\end{array}$

Long-haired Spider Monkey, White-bellied Spider Monkey

Brazil [948]; Colombia [316,759]; Ecuador; Peru [420,1022]; Venezuela [316,766,767,946]

Ateles fusciceps Gray 1866

II

V $\quad 184,624,788,1098$

Brown-headed Spider Monkey

Colombia [316,759]; Ecuador; Panama [316,442]

Ateles geoffroyi Kuhl 1820

I1

V

$184,442,624,788,1098$

(except subspecies frontatus, panamensis)

Geoffroy's Spider Monkey, Black-handed Spider Monkey

Belize [526]; ?Colombia [759]; Costa Rica; El Salvador [178]; Guatemala; Honduras [703]; Mexico [906,1150]; Nicaragua; Panama

Ateles geoffroyi frontatus Gray 1842

Black-browed Spider Monkey

I V $442,624,788,1098$

Costa Rica [1122]; Nicaragua

Ateles geoffroyi panamensis Kellogg \& Goldman 1944

Red Spider Monkey

Costa Rica; Panama 
Ateles paniscus (Linnaeus 1758)

II

$\mathrm{V}$

624,1098

Black Spider Monkey

Bolivia [116]; Brazil [251,948]; French Guiana [316]; Guyana [316]; Peru [420,1022,1023]; Surinamz $[94,316,532,760]$

Brachyteles arachnoides (E. Geoffroy 1806)

1

E

$50,184,251,358,478,751,758$, $788,982,1098$

Woolly Spider Monkey, Muriqui

Brazil

Cacajao calvus (1. Geoffroy 1847)

$1 \quad \mathrm{~V}$

$184,618,788,1098$

(includes $C$. rubicundus (1. Geoffroy \& Deville 1848))

Bald Uakari, Red-and-white Uakari

Brazil $[90,91,251,948]$; Peru [1022]

Cacajao melanocephalus (Humboldt 1812)

$1 \mathrm{~V} \quad 788,1098$

Black Uakari, Black-headed Uakari

Brazil [251,948]; Colombia [316,759]; Venezuela [316,946]

Callicebus moloch (Hoffmannsegg 1807)

11

$184,497,579,788,1183$

(includes C. brunneus (Wagner 1842), C. caligatus (Wagner 1842), C. cinerascens (Spix 1823), C. cupreus (Spix 1823), C. donacophilus (d'Orbigny 1835), C. dubius, C. hoffmannsi Thomas 1908, C. modestus Lönnberg 1939, C. oenanthe Thomas 1924, C. olallae Lönnberg 1939)

Dusky Titi

Bolivia [116,486]; Brazil [251,948]; Colombia [316,490]; Ecuador; Paraguay [915]; Peru [1022,1023]

Callicebus personatus (E. Geoffroy 1812) $\quad 11 \quad$ E $\quad 251,758,788,1098$

Masked Titi

Brazil

Callicebus torquatus (Hoffmannsegg 1807)

II

$184,788,1183$

Widow Titi

Brazil [251,948]; Colombia [316,490]; ?Ecuador; Peru [1022]; Venezuela [316,946]

Cebus albifrons (Humboldt 1812)

11

$184,788,1183$

Brown Pale-fronted Capuchin, White-fronted Capuchin

Bolivia [486]; Brazil [251,948]; Colombia [316,490]; Ecuador; ?Guyana [788]; Peru [1022, 1023]; Tinidad and Tobago [13]; Venezuela $[316,946]$

Cebus apella (Linnaeus 1758)

11

$184,788,1183$

Black-capped Capuchin, Tufted Capuchin

Argentina [124,697,699,701,915]; Bolivia [486]; Brazil [251,948]; Colombia [316,490]; Ecuador; ?French Guiana; Guyana [316,776]; Paraguay; Peru [1022, 1023]; Suriname [94,316,353,532,760]; Venezuela [316,946]

Cebus capucinus (Linnaeus 1758)

11

$184,788,1183$

White-faced Capuchin, White-throated Capuchin

?Belize [526,715,716]; Colombia [316,490]; Costa Rica [341, 1122]; Ecuador [184]; Honduras [703]; Nicaragua;

Panama; Venezuela

Cebus olivaceus Schomburgk 1848

11

$184,788,1183$

(=C. nigrivittatus Wagner 1848)

Weeper Capuchin

Brazil [948]; ?French Guiana; Guyana [316,776]; ?Peru (ex); Suriname [94,316,532,760]; Venezuela $[316,319,946]$ 
Chiropotes albinasus (1. Geoffroy \& Deville 1848) White-nosed Saki, Red-nosed Saki

Brazil

Chiropotes satanas (Hoffmannsegg 1807)

II

184,788

(except subspecies saianas)

Bearded Saki, Black Saki

Brazil [251,948]; French Guiana; Guyana [316,776]; Suriname [94,316,353,532,760]; Venezuela [316,946]

Chiropotes sasanas satanas (Hoffmannsegg 1807) Il $\quad$ E $\quad 184,568,571,788,1098$

Southern Bearded Saki, Black Saki

Brazil

Lagothrix flavicauda (Humboldt 1812)

$1 \quad$ E

$670,788,1022,1098$

(= L. hendeei Thomas 1927)

Yellow-tailed Woolly Monkey

?Ecuador; Peru

Lagothrix lagothricha (Humboldt 1812)

11

V

$184,788,1098$

Common Woolly Monkey, Humboldt's Woolly Monkey

Bolivia [1 16]; Brazil [251,948]; Colombia [316,759]; Ecuador; Peru [1022,1023]

Pithecia albicans Gray 1860

11

$251,494,569,788,948,1010$

Black-and-white Saki, Buffy Saki

Brazil

Pithecia hirsuta Spix 1823

11

494

Shaggy Saki

Bolivia [1198]; Brazil [948]; Colombia [316,759]; Ecuador; Peru [1022,1023]

Pithecia monachus (E. Geoffroy 1812)

11

$184,494,496,788,1183$

(includes $P$. aequatorialis, $P$. irrorata Gray 1844)

Monk Saki, Red-bearded Saki

Bolivia [486]; Brazil [251,948]; Colombia [490]; Ecuador; Peru [1022]

Pithecia pithecia (Linnaeus 1766)

11

$184,494,788,1183$

Pale-headed Saki, White-faced Saki

Brazil [251,948]; ?French Guiana [1183]; Guyana [316,776]; Suriname [94,316,353,532,760]; Venezuela $[316,946]$

$\begin{array}{lllll}\text { Saimiri oerstedii Reinhardt } 1872 & \text { I } & \text { E } & 788,1098\end{array}$

Central American Squirrel Monkey, Red-backed Squirrel Monkey

Costa Rica [728,1122]; Panama

Saimiri sciureus (Linnaeus 1758)

I] $\quad-\quad 184,788$

(includes S. boliviensis (d'Orbigny 1834), S. ustus (1. Geoffroy 1844))

Common Squirrel Monkey

Bolivia [116,486]; Brazil [251,854,948]; Colombia [316,490]; Ecuador; French Guiana [316]; Guyana [316,776]; ?Paraguay [757]; Peru [1022]; Suriname [94,316,353,532,760]; [?USA]; Venezuela [316,946]

Saimiri vanzolinii Ayres 1985

I1

89

Brazil 


\section{Family CERCOPITHECIDAE}

Allenopithecus nigroviridis (Pocock 1907)

II

K $\quad 443,649,789,1183$

(= Cercopithecus nigroviridis)

Allen's Swamp Monkey

?Angola [514]; Congo [1125]; Zaire [1125]

Cercocebus agilis Milne-Edwards 1886

II

$443,724,789,1183$

(=C. galeritus agilis)

Agile Mangabey

Cameroon [377]; Central African Republic [194,257]; Congo [691]; Equatorial Guinea [950]; Gabon [141,691];

Zaire $[473,991]$

Cercocebus albigena (Gray 1850)

II -

$435,443,724,789,1183$

(except subspecies aterrimus)

Grey-cheeked Mangabey, White-cheeked Mangabey

Angola [499,518]; Burundi [514]; Cameroon [377]; Central African Republic [194]; Congo [691]; Equatorial Guinea [108]; Gabon [141,377,691]; ?Kenya [443]; ?Nigeria [514]; Rwanda [40]; Tanzania [443]; Uganda [120]; Zaire [233,473,896,991]

Cercocebus albigena aterrimus (Oudemans 1890) $\quad$ II $\quad$ K $\quad 516,649$

(= C. aterrimus)

Black Mangabey

Angola; Zaire

Cercocebus galeritus galeritus Peters 1879

I E

$27,427,443,511,512,649,706$, 724,789

Tana River Mangabey

Kenya

Cercocebus galeritus 'sanjei' Homewood \& Rodgers 1981

Sanje Mangabey

11

E $\quad 513,649,938$

Tanzania

Cercocebus torquatus (Kerr 1792)

II $\quad$ V $443,649,724,789,1183$

(includes C. atys (Audebert 1797))

Collared Mangabey, Sooty Mangabey, Red-capped Mangabey, White-collared Mangabey

Cameroon [374]; Congo [691]; Côte d'lvoire [155,1070,1178]; Equatorial Guinea: Rio Muni [952]; Gabon [141,691]; Ghana [84]; Guinea [937,1074]; Liberia [14,1130]; Nigeria [450,824]; Senegal [305,1047]; Sierra Leone $[263,459,1084]$

Cercopithecus aethiops (Linnaeus 1758)

II

$443,518,724,789,1014,1018$, 1183

(includes C. pygerythrus (F. Cuvier 1821), C. sabaeus (Linnaeus 1766), C. tantalus (Ogilby 1841))

Savanna Monkey, Green Monkey, Vervet Monkey, Grivet Monkey, Tantalus Monkey

Angola [499]; [Barbados [517,1119]]; Benin [978]; Botswana [1001,1020]; Burkina Faso [944]; Burundi [1 128, 1 179]; Cameroon [557]; [Cape Verde [794]]; Central African Republic [376,689]; Chad [376,689]; Congo [691]; Côte d'Ivoire [1070]; Equatorial Guinea; Ethiopia [245,1192]; ?Gabon [141,376]; Gambia [438, 1039]; Ghana [84,152]; Guinea [155]; Kenya [1048]; Liberia [14,628]; Malawi [78,452,1066]; Mali [975]; Mauritania [376]; Mozambique [255,1017]; Namibia [376]; Niger [872]; Nigeria [940]; Rwanda; [Saint Kitts [1119]]; Senegal [305,306]; Sierra Leone [459,936]; Somalia [263,337,367]; Scuth Africa [1020]; Sudan [503,996]; Swaziland [1020]; Tanzania [1067]; Togo [84]; Uganda [120]; Zaire [991]; Zambia [75]; Zimbabwe [1177] 

1183

Schmidt's Guenon, Redtail Monkey, Black-cheeked White-nosed Monkey Angola [499]; ?Burundi; Cameroon [759]; Central African Republic [194]; Kenya [611]; Rwanda [40]; Sudan [503,621]; Tanzania [587]; Uganda [120,611]; Zaire [233,473,759,896,991]; Zambia [75]

Cercopithecus campbelli Waterhouse 1838

Campbell's Guenon, Campbell's Monkey

Côte d'Ivoire [1070,1178]; Gambia (ex?) [438,1039]; Ghana [672,937]; Guinea [672,937]; Guinea-Bissau [672]; Liberia [628]; Senegal [305,306]; Sierra Leone $[263,459,1075]$

Cercopithecus cephus (Linnaeus 1758)

Moustached Monkey

Angola [107,5I8]; Cameroon [377]; Central African Republic [514,759]; Congo [691]; Equatorial Guinea [108, 182]; Gabon [141,691]; Zaire [991]

Cercopithecus diana (Linnaeus 1758)

I $\quad$ V $443,649,724,789,1183$

(includes C. roloway (Schreber 1774)

Diana Guenon, Diana Monkey

Côte d'Ivoire [768,1070,1178]; Ghana [84]; Guinea [937]; Liberia [14,49,628]; Sierra Leone $[263,581,683]$; Togo

Cercopithecus dryas Schwarz 1932

II $\quad-\quad 1183$

Dryas Guenon, Dryas Monkey

Zaire

Cercopithecus erythrogaster Gray 1866

II $\quad E$

$443,649,724,789,1183$

Red-bellied Guenon, Red-bellied Monkey, White-throated Guenon

?Benin [978]; Nigeria [155,822,824]; Togo

Cercopithecus erythrotis Waterhouse 1838

II

$\mathrm{V}$

$443,649,724,789,1183$

(except subspecies sclateri)

Russeteared Guenon, Red-eared Monkey

Cameroon [155,377]; Equatorial Guinea: Fernando Po [155]

Cercopithecus erythrotis sclateri (Pocock 1904)

II

E

649

White-throated Guenon

Nigeria $[19,155]$

Cercopithecus hamlyni Pocock 1907

II $\quad$ V

$443,649,724,789,1183$

Owl-faced Guenon, Owl-faced Monkey

Rwanda [65,899]; Uganda [1198]; Zaire $[233,473,895,896,899]$

Cercopithecus lhoesti Sclater 1899

II

V

$435,443,648,724,789,1183$

(includes $C$. thomasi)

(except subspecies preussi)

L'Hoest's Monkey, Mountain Monkey

Burundi [899,1128]; Rwanda [322,899]; Uganda [120,611]; Zaire [233,473,899]

Cercopithecus lhoesti preussi (Matschie 1898)

II

E

$443,649,724,789,1183$

(=C. preussi)

Preuss's Guenon, Preuss's Monkey

Cameroon [373]; Equatorial Guinea: Fernando Po [691] 
(includes C. albogularis (Sykes 1831))

Blue Monkey, Diademed Monkey, Sykes' Monkey

Angola [499,518]; Burundi [322,1128]; Ethiopia [1 192]; Kenya [61 I ,706]; Malawi [78,452, 1066]; Mozambique [255,1017]; Rwanda [26,86,677]; Somalia [337,367]; South Africa [725,1020]; Sudan [996]; Swaziland; Tanzania [938,1067]: including Zanzibar [771]; Uganda [120]; Zaire [233,473,896,991]; Zambia [75]; Zimbabwe [219]

Cercopithecus mona (Schreber 1774)

Mona Monkey

Benin [978]; Cameroon (557]; Ghana [152]; [Grenada] [791]; Nigeria [824]; [Saint Kitts]; Sāo Tome and Principe [365]; Togo [84]

Cercopithecus neglectus Schlegel 1876

Il

$435,724,789,1183$

de Brazza's Monkey

Angola [518]; Cameroon [759]; Central African Republic [194,257]; Congo [691]; Equatorial Guinea [108, 182]; Ethiopia [1192]; Gabon [141,691]; Kenya [165,326,779]; Sudan [514]; Uganda [120]; Zaire [233,473,759,991]

Cercopithecus nictitans (Linnaeus 1766)

11

$443,724,789,1183$

White-nosed Guenon, Putty-nosed Monkey, Spot-nosed Guenon, Greater White-nosed Monkey

Angola [518]; Benin [978]; Cameroon [557]; Central African Republic [194,689]; Congo [691]; Côte d'Ivoire [1070,1178]; Equatorial Guinea: including Fernando Po [182]; Gabon [141,691]; Liberia [14,628]; Nigeria [765,824]; Sierra Leone [451]; Togo; Zaire [991]

Cercopithecus petaurista (Schreber 1774)

I1

$443,724,789,1183$

Lesser White-nosed Guenon, Spot-nosed Monkey, Lesser Spot-nosed Guenon, Lesser White-nosed Monkey Benin [978]; Côte d'Ivoire [1070,1178]; Gambia; Ghana [84]; Guinea [155,937]; Guinea-Bissau [305]; Liberia [628]; Senegal [305]; Sierra Leone [263,459]; Togo

Cercopithecus pogonias Bennett 1833

11

$443,724,789,1183$

Golden-bellied Guenon, Golden-bellied Monkey, Crowned Guenon, Crowned Monkey

Cameroon [377]; Central African Republic [194,1074]; Congo [691]; Equatorial Guinea [108, 182, 1093]; Gabon [141,691,759]; Nigeria (ex?) [19,823]; Zaire [759]

Cercopithecus salongo Thys van Audenaerde 1977

Salongo Guenon, Zaire Diana Monkey

?Zaire

Cercopithecus solatus Harrison 1988

Sun-tailed Guenon, Sun-tailed Monkey

Gabon [141]

Cercopithecus talapoin Schreber 1774

(= Miopithecus talapoin)

\section{Talapoin}

Angola [518]; *Cameroon [557]; [Canary Islands]; Congo [691]; *Equatorial Guinea [182]; *Gabon [141,691]; Zaire [991]

(* The populations of these countries may belong to an undescribed species [514])

Cercopithecus wolfi Meyer 1891

II

II

II $\quad$ V $\quad 470,649$

(=C. pogonias wolfi)

(includes C. denti (Thomas 1907))

Wolf's Monkey

Angola [672]; Central African Republic [689]; Rwanda [789]; Uganda [611,672]; Zaire [233,473,672,896] 
Colobus angolensis Sclater 1860

Angolan Black-and-White Colobus, Angolan Colobus

Angola [518]; Burund; [898,1128]; Congo [691]; Kenya [611]; Malawi [790]; Rwanda [40,543]; Tanzania [938, 1067]; Uganda [120]; Zaire [899]; Zambia [75]

Colobus badius (Kerr 1792)

Il V

$156,382,649,724,790,899,1038$, 1183

( = Procolobus badius)

(except subspecies temminckii, waldroni)

Western Red Colobus

Côte d'Ivoire [84,899,1178]; Guinea [937]; Liberia [14,899,1049]; Sierra Leone [263,459]

$\begin{array}{llll}\text { Colobus badius temminckii Kuhl } 1820 & 11 & \text { R } & 649,790\end{array}$

(= Procolobus temminckii)

Temminck's Red Colobus

Gambia [438,1039, 1049]; Guinea; Guinea-Bissau [790]; Senegal [305, 1049]

Colobus badius waldroni Hayman 1936

Il $\quad$ E $\quad 649,790$

(= Procolobus badius waldroni)

Miss Waldron's Bay Colobus

Côte d'lvoire; Ghana [153]

Colobus guereza Rūppell 1835

11

$300,301,435,443,650,724,790$,

(=C. abyssinicus Oken 1816, invalid) $820,899,1183,1192$

Guereza, Magistrate Colobus, Eastern Black-and-White Colobus

Cameroon [557]; Central African Republic [194,689]; Chad [689]; Congo [691]; Equatorial Guinea [759]; Ethiopia [245,1192]; Gabon [141,691]; Kenya [611]; Nigeria [19,820]; Rwanda [322,820]; Sudan [503]; Tanzania [611]; Uganda [120]; Zaire [473,896,991]

$\begin{array}{llll}\text { Colobus pennantii Waterhouse } 1838 & \text { II } & \text { E } & 649,790\end{array}$

(= Procolobus pennantii, $C$. badius pennantii)

(except subspecies bouvieri, ellioti, foai, gordonorum, kirki, oustaleti, tephrosceles, tholloni)

Eastern Red Colobus

Equatorial Guinea: Femando Po $[25,899,1049]$

Colobus pennantii bouvieri (Rochebrune 1887)

II $\quad$ E $\quad 649$

(= Procolobus pennantii bouvieri, $C$. badius bouvieri)

Bouvier's Red Colobus

Congo [899]

Colobus pennantii ellioti Dollman 1909

Il

K

649

(= Procolobus rufomitratus ellioti, $C$. badius ellioni)

Elliot's Red Colobus

Uganda (ex?) [882]; Zaire

Colobus pennantii foai Pousargues 1899

K

649

(= Procolobus rufomitratus foai, $C$. badius foai)

Foa Red Colobus

Zaire

Colobus pennaniii gordonorum (Matschie 1900)

11

E

$27,435,443,649,938$

(= Procolobus gordonorum, C. badius gordonorum)

Uhehe Red Colobus, Gordon's Red Colobus

Tanzania 
Colobus pennantii kirkii Gray 1868

1

E

$27,435,443,649,724,790$

(= Procolobus kirkii, C. badius kirkii, C. kirkii)

Zanzibar Red Colobus, Kirk's Colobus

Tanzania: [Pemba Island] [1006], Zanzibar [899]

Colobus pennantii oustaleti Trouessart 1906

11

K

649

(= Procolobus rufomitratus oustaleti, C. badius oustaleti)

Oustalet's Red Colobus

Central African Republic [759]; ?Sudan [724,899]; Zaire

Colobus pennantii tephrosceles Elliot 1907

II V

649

(= Procolobus rufomitratus tephrosceles, C. badius tephrosceles)

Uganda Red Colobus

Burundi [899,991]; Rwanda; Tanzania [1050]; Uganda [120,1050]

Colobus pennantii tholloni Milne-Edwards 1886

$11 \quad \mathrm{~K}$

649

(= Procolobus rufomitratus tholloni, C. badius tholloni)

Thollon's Red Colobus

Zaire

Colobus polykomos (Zimmermann 1780)

11

$443,724,690,790,899,1183$

(includes $C$. vellerosus (1. Geoffroy 1834))

King Colobus, Western Black-and-White Colobus

Benin [978]; Côte d'lvoire [84,899,1178]; Gambia [899]; Ghana [84,899,1178]; Guinea [155,937]; Guinea-Bissau [305, 764]; Liberia [14,628]; Nigeria [940]; Senegal [ 155,305]; Sierra Leone [263,459,936]; Togo $[84,899]$

Colobus preussi (Matschie 1900)

11 E $27,443,649,724,790$

(=C. badius preussi, Procolobus pennanti preussi)

Preuss's Colobus, Preuss's Red Colobus

Cameroon; Nigeria (ex?) [19]

Colobus rufomitratus Peters 1879

I V

$27,435,443,649,724,790,1050$, 1061

(= C. badius rufomitratus, Procolobus rufomitratus rufomitratus)

Tana River Colobus, Eastern Red Colobus

Kenya

Colobus satanas Waterhouse 1838

II

E

$27,443,649,724,790,899,1183$

Black Colobus

Cameroon [820]; Congo [691]; Equatorial Guinea [952]: including Fernando Po [724]; Gabon [141,691]

Colobus verus Van Bencden 1838

II R $\quad 27,649,724,790,1183$

(= Procolobus verus)

Olive Colobus, Van Beneden's Colobus

?Benin [978]; Côte d'Ivoire [154,1178]; Ghana [154]; Guinea [154,155]; Liberia [14,154]; Nigeria [19,737, 822];

Sierra Leone [154,263]; ?Togo

Erythrocebus patas (Schreber 1775)

11

$435,443,724,789,1183$

(= Cercopithecus patas)

Patas Monkey

Benin [978]; Burkina Faso [944]; Cameroon [557]; Central African Republic [689]; Chad [689]; Côte d'Ivoire [1070]; Ethiopia [1192]; Gambia [438,1039]; Ghana [84]; Guinea; Guinea-Bissau; Kenya [611]; Mali [975]; Mauritania; Niger [873]; Nigeria [940]; Senegal [305,307]; Sierra Leone [1171]; ?Somalia [611]; Sudan [503,996]; Tanzania [1067]; Togo [84]; Uganda [120]; Zaire [991] 
Macaca arctoides (1. Geoffroy 1831)

11

$336,789,1183$

(= M. speciosa Blyth 1875 )

Stump-tailed Macaque, Bear Macaque

Bangladesh [601,602,603,604,605]; Cambodia [864]; China [363,1072,1202]; India [38,763]; Lao P.D.R. [271,417]; Malaysia: Peninsular Malaysia [707,721]; Myanmar [1109]; Thailand [96,151]; Viet Nam $[684,837,853,1025]$

Macaca assamensis (McClelland 1840) Il $\quad$ - $\quad 336,361,789,1183$

Assam Macaque

Bangladesh [393,601,602,603,604,605,970]; Bhutan; China [345,1072,1202]; India [762,763]; Lao P.D.R. [271]; Myanmar [1109]; Nepal [756]; Thailand [151]; Viet Nam [261,684,837,853,1025]

Macaca cyclopis (Swinhoe 1863)

$11 \quad$ V $\quad 336,878,879,1072,1183,1202$

Taiwan Macaque, Formosan Rock Macaque

[Japan]; Taiwan

Macaca fascicularis (Raffles 1821)

11

$217,336,686,723,789,1141$,

(= M. irus Cuvier 1818, M. cynamolgus (Schreber 1775))

Crabeating Macaque, Cynomolgus Monkey, Long-tailed Macaque

Bangladesh [601,602,603,604,605,970]; Brunei [119,170]; Cambodia; [Hong Kong] [709]; lndia: Nicobar lslands [38,278,763]; Indonesia [250,752,1009]; ?Lao P.D.R.; Malaysia: Peninsular Malaysia, Sabah [266], Sarawak [707,721]; [Mauritius] [1063, 1064]; Myanmar [1109]; Palau [880]; Philippines [892]; Singapore [469]; Thailand [96,151]; [?USA]; Viet Nam [684,837,853,1025]

Macaca fuscata (Blyth 1875)

$336,594,1141,1183$

(= M. speciosa I. Geoffroy 1826)

(except subspecies yakui)

Japanese Macaque

[Hong Kong]; Japan

Macaca fuscata yakui Kuroda 1941

Il

E $\quad 1034$

Yakushima Macaque

Japan

Macaca maura F. Schinz 1825

11

V

336

Moor Macaque

Indonesia: Sulawesi

Macaca mulatta (Zimmermann 1780)

II

$336,789,1027,1141,1183$

Rhesus Macaque, Rhesus Monkey

Afghanistan [476]; Bangladesh [393,601,602,603,604,605,970]; Bhutan; China [1072, 1202, 1204]; Hong Kong [709]; India [762,763,1026]; Lao P.D.R. [271,417,837]; Myanmar [1028,1109]; Nepal [756]; Pakistan [797,930]; Thailand [151,216]; [USA]; Viet Nam [261,684,837,853,1025]

Macaca nemestrina (Linnaeus 1766)

11

$336,723,789,1183$

(except subspecies pagensis)

Pigtail Macaque

Bangladesh [393,601,602,603,604,605,970]; Brunei [1 19, 170]; ?Cambodia; China [1072, 1202]; India [38,763]; Indonesia: Bangka, Kalimantan [1009], Sumatra [250]; Lao P.D.R. [271,359]; Malaysia: Peninsular Malaysia, Sabah [266], Sarawak [707,721]; Myanmar [1109]; Singapore (ex) [469]; Thailand [151,216]; Viet Nam $[684,853,1025]$

Macaca nemestrina pagensis Miller 1903 II $\quad$ E $27,1168,1183$

(= M. pagensis)

Mentawai Macaque

Indonesia: Mentawai Islands, Siberut, Sipora, North and South Pagai Islands 
Macaca nigra (Desmarest 1822)

II

$336,789,1183$

(includes $M$. nigrescens)

Sulawesi Macaque, Celehes Black Macaque, Gorontalo Macaque

Indonesia: Bacan, Sulawesi

Macaca ochreata (Ogilby 1841)

II

336

(except subspecies brunnescens)

Booted Macaque

Indonesia: Sulawesi

Macaca ochreata brunnescens Matschie 1901

(= M. brunnescens)

II $\quad \mathrm{K} \quad 426$

Muna-Butung Macaque

Indonesia: Butung, Muna

Macaca radiata (E. Geoffroy 1812)

II

$99,100,336,762,763,789,893$, 1183

\section{Bonnet Macaque}

India

Macaca silenus (Linnaeus 1758)

I

E $27,38,45,99,100,132,336,421$, $575,576,592,763,789$

Lion-tailed Macaque

India [1141]

Macaca sinica (Linnaeus 1771)

II

$336,789,865,1183$

Toque Macaque

Sri Lanka

Macaca sylvanus (Linnaeus 1758)

II $\quad$ V

$27,333,335,336,724,789,1183$, 1198

Barbary Macaque, Barbary Ape

Algeria [729, 1080]; [GibraItar [268,818]]; Morocco [183,332,334,1080]

Macaca thibetana (Milne-Edwards 1870)

II

K

$336,362,363,994,1072$

Pere David's Macaque, Tibetan Macaque

China

Macaca tonkeana (Meyer 1899)

II

336

(except subspecies hecki)

Tonkean Macaque

Indonesia: Palau Malenge, Togian Islands

Macaca tonkeana hecki Matschie 1901

II

V

426

( $=M$. hecki)

Heck's Macaque

Indonesia: Sulawesi

Nasalis concolor Miller 1903

(= Simias concolor)

Pig-tailed Langur, Pagai Island Langur

Indonesia: Mentawai Islands

Nasalis Larvatus (Wurmb 1787)

Proboscis Monkey, Long-nosed Monkey

Brunei [170,673]; Indonesia: Kalimantan [135]; Malaysia: Sabah [266], Sarawak [956] 
(= P. anubis)

Olive Baboon

Benin [978,1131]; Burkina Faso [84]; Burundi [102,1128,1179]; Cameroon [84]; Central African Republic [155, 194,296]; Chad [689, 1074]; Congo [759]; Côte d'Tvoire [84,103,1070]; Ethiopia [245,1192]; Ghana [84]; ?Guinea [155]; Kenya [611]; Libya [528]; Mali [975]; ?Mauritania [102]; Niger [872]; Nigeria [1,940]; Rwanda [26]; ?Sierra Leone [459,789,936]; ?Somalia; Sudan [503]; Tanzania [1067]; Togo [84]; Uganda [120]; Zaire $[233,473,896,991]$

Papio hamadryas cynocephalus (Linnaeus 1766) II $\quad-\quad 435,443,724,789,1183$ (=P. cynocephalus)

Yellow Baboon

Angola [518]; Ethiopia [789]; Kenya [611,706]; Malawi [78,452,1066]; Mozambique [1017, 1020]; Somalia [337,367]; Tanzania [1067]; Zaire [991]; Zambia [75]

Papio hamadryas hamadryas (Linnaeus 1758)

11

R $\quad 443,649,724,789,1183$

Hamadryas Baboon

Djibouti; Egypt (ex); Ethiopia [245,1192]; Saudi Arabia [463]; Somalia [337,367]; Sudan [501]; Yemen $[463,907]$

Papio hamadryas papio (Desmarest 1820)

11

$443,724,789,1183$

(= P. papio)

Guinea Baboon, Western Baboon

Gambia [1039]; Guinea [155]; ?Guinea-Bissau [155]; ?Liberia [102]; Mali [102]; Mauritania [155]; Senegal [305,307]; Sierra Leone $[459,936]$

Papio hamadryas ursinus (Kerr 1792)

II

$724,789,1018,1183$

(= P. ursinus)

Chacma Baboon

Angola [499,518]; Botswana [1020]; Lesotho [1020]; Malawi [1066]; Mozambique [1017,1020]; Namibia [1020]; South Africa [1020]; Swaziland [1020]; Zambia [75]; Zimbabwe [1020]
Papio leucophaeus (F. Cuvier 1807)
I $\quad E$
$27,649,724,789,1183$

(= Mandrillus leucophaeus)

Drill

Cameroon; Congo (ex); Equatorial Guinea: Fernando Po [108,320]; ?Gabon [141]; Nigeria [19,823]

Papio sphinx (Linnaeus 1758)

$1 \quad \mathrm{~V}$

$429,649,724,789,1183$

(= Mandrillus sphinx)

Mandrill

Cameroon; Congo; Equatorial Guinea: Rio Muni; Gabon [141]

Presbytis comata Desmarest 1822

$11 \quad$ E $\quad 168,790$

(= P. aygula misapplied, not (Linnaeus 1758))

Grizzled Leaf Monkey, Java Leaf Monkey

Indonesia: Java

Presbytis cristata (Raffles 1821)

II

$723,790,1183$

(= Trachypithecus cristatus)

(includes Trachypithecus auratus (= P. aurata), P. pyrrhus (Horsfield 1823))

Silvered Leaf Monkey, Silvered Langur, Ehony Leaf Monkey

Brunei; Cambodia [790]; Indonesia [168,250,722]; Lao P.D.R. [271,360,837,976]; Malaysia: Peninsular Malaysia [707,721], Sabah [266,468], Sarawak; Myanmar [1109]; Thailand [151]; Viet Nam $[259,261,684,837,853,1025]$ 
Presbytis entellus (Dufresne 1797)

1

790,1183

(= Semnopithecus entellus)

(includes S. hypoleucos (Blyth 1841))

Hanuman Langur, Common Langur, Grey Langur, Entellus Langur

Bangladesh [393,601, 602,603,604,605,970]; ?Bhutan; China [1072,1202]; India [100,381,763,1055]; Nepal [573,756]; Pakistan [797,930]; Sri Lanka [318,865]

Presbytis francoisi (Pousargues 1898)

II $\quad \mathrm{E}$

$654,790,1183$

(= Trachypithecus francoisi)

(except subspecies leucocephala)

François' Leaf Monkey, François' Langur, Tonkin Leaf Monkey

China $[47,1072,1202]$; Lao P.D.R. $[271,360,976]$; Viet Nam $[261,684,837,1025]$

Presbytis francoisi leucocephala T'an 1957

$11 \quad$ E $\quad 244,654$

(= Trachypithecus leucocephalus)

White-headed Black Leaf Monkey

China

Presbytis frontata (Müller 1838)

11

$721,723,790,1183$

White-fronted Leaf Monkey, White-faced Langur

Brunei [170]; Indonesia: Kalimantan; Malaysia: Sarawak

Presbytis geei Gee 1956

(= Trachypithecus geei)

Golden Langur, Golden Leaf Monkey

Bhutan [207]; India [38]

Presbytis hosei (Thomas 1889)

11

$217,723,790$

(= P. comala hosei)

Grey Leaf Monkey, Hose's Leaf Monkey

Brunei [119]; Indonesia: Kalimantan; Malaysia: Sabah [266,468], Sarawak

Presbytis johnii (Fischer 1829)

$11 \quad$ E

$27,38,99,100,519,520,521,763$, $790,821,1183$

(= Trachypithecus johnii)

Nilgiri Langur, Black Leaf Monkey, John's Langur

India

Presbytis melalophos (Raffles 1821)

II $\quad-\quad 469,721,723,790$

(includes P. femoralis Martin 1838)

Banded Leaf Monkey, Banded Langur, Mitred Leaf Monkey

Indonesia: Batu Islands, Kalimantan [1009], Natuna Islands, Rhio Archipelago, Sumatra [250,722]; Malaysia:

Peninsular Malaysia [707,721], Sarawak; Myanmar [1109]; Singapore (ex?) [217]; Thailand [96,151]

Presbytis obscura (Reid 1837)

II

790,1183

(= Trachypithecus obscurus)

Dusky Leaf Monkey, Spectacled Leaf Monkey, Spectacled Langur

Bangladesh [603]; Lao P.D.R [417]; Malaysia: Peninsular Malaysia [707,721]; Myanmar [1109]; Thailand $[15], 216]$

Presbytis phayrei Blyth 1847

II $\quad-\quad 790,1183$

(= Trachypithecus phayrei)

Phayre's Leaf Monkey, Phayre's Langur

Bangladesh [393,601,602,603,604,605]; ?Cambodia; China [1072,1202]; India [222,762,778]; Lao P.D.R. $[271,360,976]$; Myanmar [1109]; Thailand [151]; Viet Nam [360,1025] 
Presbytis pileata (Blyth 1843)

I

790,1183

(= Trachypithecus pileatus)

Capped Langur, Capped Leaf Monkey

Bangladesh [393,601,602,603,604,605,970]; India [38,763]; Myanmar [955,1109]

Presbytis potenziani (Bonaparte 1856)

1

E $27,790,1183$

Mentawai Leaf Monkey, Mentawai Langur

Indonesia: Mentawai Islands, Pagai, Siberut, Sipora

Presbytis rubicunda (Müller 1838)

II

$721,723,790,1183$

Maroon Leaf Monkey, Maroon Langur

Brunei $[170,648]$; Indonesia: Kalimantan [154], Karimata Islands [217]; Malaysia: Sabah [266,468], Sarawak

Presbytis senex (Erxleben 1777)

11

$790,865,1183$

(= P. vetulus (Erxleben 1777), Trachypithecus vetulus)

Purple-faced Leaf Monkey

Sri Lanka

Presbytis thomasi (Collett 1893)

11

$217,250,790$

(= P. comata thomasi)

Thomas's Leaf Monkey, Thomas's Langur

Indonesia: Sumatra

Pygathrix avunculus (Dollman 1912)

I

E

$684,790,1025,1183$

(= Rhinopithecus avunculus)

Tonkin Snub-nosed Monkey

Viet Nam

Pygathrix brelichi (Thomas 1903)

I

E

$27,790,1072,1183$

(= Rhinopithecus brelichi, $R$. roxellana brelichi)

Guizhou Snub-nosed Monkey, Grey Snub-nosed Monkey, Brelich's Snub-nosed Monkey

China

Pygathrix nemaeus (Linnaeus 1771)

$1 \quad$ E $27,790,900$

(except subspecies nigripes)

Douc, Douc Langur, Red-shanked Douc Langur

China: Hainan [1072,1202]; Lao P.D.R. [271,837,855]; Viet Nam [684,837,853,1025]

Pygathrix nemaeus nigripes (Milne-Edwards 1871) I $\quad$ E $\quad 27,790,900$

Black-shanked Douc Langur

Cambodia [864]; Lao P.D.R.; Viet Nam

Pygathrix roxellana (Milne-Edwards 1870)

(= Rhinopithecus roxellanae)

I $\quad$ V $27,790,1183,1194$

(except subspecies bieti)

Golden Snub-nosed Monkey, Sichuan Snub-nosed Monkey

China $[655,877,983,994,1072,1202]$; ?India: Arunachal Pradesh, Manipur $[383,763]$

Pygathrix roxellana bieti (Milne-Edwards 1897)

1

E

791

(= Rhinopithecus bieti)

Yunnan Snub-nosed Monkey

China

Theropithecus gelada (Rũppell 1835)

II $\quad \mathrm{R} \quad 245,443,649,724,789,1183$,

Gelada Baboon

1192

Ethiopia 


\section{Family HYLOBATIDAE}

Hylobates agilis F. Cuvier 1821

1

423,723

$(=H$. lar agilis $)$

Agile Gibbon, Dark-handed Gibbon

Indonesia: Kalimantan, Sumatra [250]; Malaysia: Peninsular Malaysia [707]; Thailand [15I,1112]

Hylobates concolor (Harlan 1826)

$$
\text { I } \quad \mathrm{V} \quad 2,27,423,1183
$$

(includes $H$. leucogenys Ogilby 1840)

Black Gibbon, Crested Gibbon, White-cheeked Gibbon, Indochinese Gibbon

Cambodia [423]; China $[140,441,1072,1169,1202]$; Lao P.D.R. $[220,271,423,976]$; Viet Nam $[387,684,853,1025]$
Hylobates hoolock (Harlan 1834)
1
V $\quad 224,423,1183$

Hoolock Gibbon

Bangladesh [393,601,602,603,604,605,970]; China [441,1072,1202]; India [38,222,763]; ?Lao P.D.R. [271,417]; Myanmar [955,1109]

Hylobates klossii (Miller 1903)

Mentawai Gibbon, Kloss's Gibbon, Dwarf Gibbon

I

E $27,423,1090,1167,1183$

Indonesia: Mentawai Islands

Hylobates lar (Linnaeus 1771)

$1 \quad-\quad 423,1183$

Common Gibbon, Lar Gibbon, White-handed Gibbon

China [441, 1072,1202]; Indonesia: Sumatra [250]; Lao P.D.R. [271,417]; Malaysia: Peninsular Malaysia [707],

Sabah, Sarawak; Myanmar [955,1109]; Thailand [151]

$\begin{array}{llll}\text { Hylobates moloch (Audebert 1798) } & \text { I } & \text { E } & 27,423,589\end{array}$

(= H. lar moloch)

Javan Gibbon, Silvery Gibbon

Indonesia: Java

Hylobates muelleri Martin 1841

(= H. lar muelleri)

Bornean Gibbon, Grey Gibbon, Müller's Gibbon

Brunei $[119,170]$; Indonesia: Kalimantan [1009]; Malaysia: Sabah [266], Sarawak

Hylobates pileatus (Gray 1861) I $\quad$ E $\quad 27,423,1183$

(= H. lar pileatus)

Pileated Gibbon, Crowned Gibbon, Capped Gibbon

Cambodia [220,410]; Lao P.D.R. [272,976]; Thailand [96, 151,216]; Viet Nam (ex?) [853,1025]

$\begin{array}{llll}\text { Hylobates syndactylus (Raffles 1821) } & \text { I } & - & 721,1183\end{array}$

(= Symphalangus syndactylus)

Siamang

Indonesia: Sumatra [220,250]; Malaysja: Peninsular [606,707]

Family PONGIDAE

Gorilla gorilla Savage \& Wyman 1847

I V $\quad 27,443,455,649,724$

(except subspecies beringei, graueri)

Gorilla

Angola [518,531]; Cameroon [181,375,434]; Central African Republic [194, 1030]; Congo [375]; Equatorial Guinea [951]; Gabon [141,1107]; Nigeria [19,375,456,698]; Zaire [780] 
Gorilla gorilla beringei Matschie 1903

1

E $27,649,1152$

Mountain Gorilla

Burundi (ex?) [1128]; Rwanda [327,1153]; Uganda [120,454]; Zaire: Virunga Volcano region [87,88,327, $389,457,896,1002]$

Gorilla gorilla graueri Matschie 1914

I $\quad$ E $\quad 649,780$

Eastern Lowland Gorilla

Zaire

Pan paniscus Schwarz 1929

I $\quad$ V $\quad 649,724$

Dwarf Chimpanzee, Pygmy Chimpanzee, Bonobo

?Congo [691]; Zaire [233,759]

Pan troglodytes (Gmelin 1788)

$1 \quad \mathrm{~V}$

$27,435,443,649,724,1087$

(except subspecies verus)

Chimpanzee

Angola [518,531]; Burundi [1127,1128,1179]; Cameroon [1088]; Central African Republic [194, 1088]; Congo [1088]; Equatorial Guinea [1088]; Gabon [141,1107]; Nigeria [19,35, 1088]; Rwanda (ex?) [35, 1088]; Sudan [503]; Tanzania [1088]; Uganda [10,120,1088]; Zaire [233,473,896,1088]

Pan troglodytes verus Schwarz 1934

1

E $\quad 649$

West African Chimpanzee

Benin (ex) [978]; Burkina Faso (ex) [1088]; Côte d'Ivoire [1088]; [Gambia [35,705]]; Ghana [1088]; Guinea [1088]; Guinea-Bissau (ex?) [35,1088]; Liberia [14,49,1088]; Mali [1088]; Senegal [305,718]; Sierra Leone $[263,459,1088]$; Togo (ex) $[35,1088]$

Pongo pygmaeus (Linnaeus 1760)

I $\quad$ E $27,472,858$

Orang-utan

?Brunei [723]; Indonesia: Kalimantan [1009], Sumatra [157,159,926,927]; Malaysia: Sabah [266,468], Sarawak

\section{Order EDENTATA $\quad(=$ XENARTHRA $)$}

\section{Family MYRMECOPHAGIDAE}

Myrmecophaga tridactyla Linnaeus 1758

$11 \quad \mathrm{~V} \quad 1098,1161$

(= M. jubata)

\section{Giant Anteater}

Argentina [124,184,699,701]; Belize (ex?) [442]; Bolivia [184]; Brazil [184,251]; Colombia [184]; Costa Rica [442,1122]; ?Ecuador [184]; El Salvador (ex) [442]; French Guiana [184]; Guatemala (ex) [442]; Guyana [184]; Honduras [442,703]; Nicaragua [442]; Panama [442]; Paraguay [184]; Peru [184,420,1122]; Suriname [184,532]; Unuguay $[958,1161]$; Venezuela $[184,319,766]$

Tamandua tetradactyla (Linnaeus 1758)

III GT

$700,1159,1161$

(includes T. mexicana (Saussure 1860))

Tamandua, Collared Anteater, Lesser Anteater

Argentina [184,699,701]; Belize [1161]; Bolivia [184]; Brazil [184,251,702,744,869]; Colombia [316]; Costa Rica [1161]; Ecuador [1076]; El Salvador [178,1161]; French Guiana [184,700]; Guatemala [1161]; Guyana [184]; Honduras [703,1161]; Mexico [354,906,1161]; Nicaragua [1161]; Panama [1161]; Paraguay [184,1 162]; Peru [85,184,420]; Suriname [184,532]; Uruguay; Venezuela [63, 184,319,448,766] 
Family BRADYPODIDAE

Bradypus torquatus Desmarest 1816

E

$184,251,868,1098,1161$

Maned Sloth

Brazil

Bradypus variegatus Schinz 1825

II

700,1161

(includes $B$. boliviensis, $B$. griseus, B. infuscalus)

Brown-throated Sloth

Argentina [184]; Bolivia [184]; Brazil [184,251]; Colombia [184,316]; Costa Rica [442]; Ecuador [184]; ?El

Salvador [442]; Honduras [442,703]; Nicaragua [442]; Panama [442]; Paraguay [184]; Peru [184,420];

Venezuela $[184,766]$

Family CHOLOEPIDAE ( = MEGALONYCHIDAE)

Choloepus hoffmanni Peters 1859

$111 \mathrm{CR} \quad-\quad 700,1161$

Hoffmann's Two-toed Sloth

Bolivia [1198]; Brazil [184]; Colombia [184,316]; Costa Rica [442]; Ecuador [184]; Honduras [703]; Nicaragua [442]; Panama [442]; Peru $[85,184,420]$; Venezuela $[448,766]$

\section{Family DASYPODIDAE}

Cabassous centralis (Miller 1899)

III CR - $\quad 442,700,1161$

Northern Naked-tailed Armadillo

Belize [715]; Colombia [1160]; Costa Rica [1160]; Ecuador [316]; El Salvador; Guatemala [1160]; Honduras [703,1160]; Nicaragua [1160]; Panama [1160]; Venezuela [316,1160]

Cabassous tatouay (Desmarest 1804)

III UY -

$184,700,1160,1161$

(= C. gymnurus)

Greater Naked-tailed Armadillo

Argentina [124]; Brazil; Paraguay; Uruguay

Chlamyphorus retusus (Burmeister 1863)

K $\quad 184,1098,1161$

(= Burmeisteria relusa)

Greater Pichi Ciego, Burmeister's Armadillo, Chacoan Fairy Armadillo

Argentina [124,632,699]; Bolivia [1198]; Paraguay

Chlamyphorus truncatus Harlan 1825

K $\quad 184,1098,1161$

Lesser Pichi Ciego, Pink Fairy Armadillo, Lesser Fairy Armadillo

Argentina [124]

Priodontes maximus Kerr 1792

$1 \quad \mathrm{~V} \quad 184,1098,1161$

(=P. giganteus Geoffroy 1803)

Giant Armadillo

Argentina [124,699]; Bolivia [1198]; Brazil [251]; Colombia [316]; French Guiana [316]; Guyana [316,701]; Paraguay; Peru [420]; Suriname [532]; Venezuela [316]

Tolypeutes tricinctus (Linnaeus 1758)

Id

$184,251,1098,1161$

Brazilian Three-banded Armadillo

Brazil 


\section{Order PHOLIDOTA}

\section{Family MANIDAE}

Manis crassicaudata Gray 1827

II $\quad-\quad 514$

Indian Pangolin

Bangladesh [602,603,604,970]; China; India [38,99,884]; Pakistan [797,930]; Sri Lanka [865]

$\begin{array}{lll}\text { Manis gigantea Illiger } 1815 & \text { III GH } \quad-\quad 724\end{array}$

(= Phataginus gigantea)

Giant Ground Pangolin, Giant Pangolin

Angola [518]; Benin [978]; Burkina Faso; ?Burundi; Cameroon [557]; ?Central African Republic; Chad; Congo [691]; ?Côte d'Ivoire; Equatorial Guinea [108]; Gabon [839]; ?Gambia; Ghana [156]; ?Guinea; ?Guinea-Bissau; Kenya; Liberia [14,628]; ?Mali; ?Mauritania; Niger; Nigeria [1,940]; Rwanda; Senegal [307]; Sierra Leone [1086]; Sudan [503]; ?Tanzania; ?Togo; Uganda [120]; Zaire [233,896,991]

Manis javanica Desmarest $1822 \quad$ II $\quad 723$

Malayan Pangolin

?Bangladesh [602,603]; Brunei; Cambodia; ?China; Indonesia [217,934,1009]; Lao P.D.R. [271, 417]; Malaysia: Peninsular, Sabah [266], Sarawak; Myanmar [955]; Philippines: Palawan; Singapore (ex?); Thailand [96,151]; Viet Nam $[837,853,1025]$

Manis pentadactyla Linnaeus 1758

II $\quad-\quad 544$

Chinese Pangolin

?Bangladesh [602]; Cambodia [410]; China [1169]; Hong Kong [709]; India [38]; Lao P.D.R. [271,417,837]; Myanmar [955]; Nepal [756]; Taiwan; Thailand [96,151]; Viet Nam [837,853,1025]

Manis temminckii Smuts 1832

$1 \quad-\quad 724,1051$

(= Phataginus temminckii)

Temminck's Ground Pangolin, Cape Pangolin

Angola [518]; Botswana; Central African Republic [689]; Chad [689]; Ethiopia; Kenya [611]; Malawi [78,452]; Mozambique [1017]; Namibia; Rwanda [1020]; Somalia; South Africa [725]; Sudan [503]; Tanzania [611]; Uganda [120,611]; Zaire; Zambia; Zimbabwe

Manis tetradactyla Linnaeus 1766

III GH $\quad-\quad 724$

(= M. longicaudata, Phataginus tetradactyla)

Long-tailed Pangolin, Black-bellied Pangolin

Angola; ?Burkina Faso [944]; ?Burundi; Cameroon [557]; ?Central African Republic; Congo [691]; Côte d'Ivoire [894]; Equatorial Guinea [182]; Gabon [839]; Gambia; Ghana; ?Guinea; Liberia [14,628]; ?Mali; Nigeria [940]; ?Rwanda; Senegal; Sierra Leone [263]; Sudan; ?Togo; Uganda [120]; Zaire [233,896,991]

Manis tricuspis Rafinesque $1821 \quad 111 \mathrm{GH} \quad-\quad 77,724$

(= Phataginus tricuspis)

Tree Pangolin, White-bellied Pangolin, Three-cusped Pangolin

Angola [499,518]; Benin [978]; ?Burkina Faso [944]; Cameroon [557]; ?Central African Republic; Congo [691];

Côte d'Ivoire [894]; Equatorial Guinea [182,1093]; Gabon [840]; ?Gambia; Ghana [83]; Guinea [937];

?Guinea-Bissau; Kenya [611]; Liberia [14,628]; Nigeria [940]; ?Senegal; Sierra Leone [263,1086]; Sudan [503];

Tanzania [611]; ?Togo; Uganda [611]; Zaire [233,896,991]; Zambia [75] 


\section{Order LAGOMORPHA}

Family OCHOTONIDAE

Ochotona koslowi (Buchner 1894)

Kozlov's Pika

China

Ochotona muliensis Pen \& Feng 1962

Muli Pika

China

Prolagus sardus Wagner 1832

Sardinian Pika

Sardinia (ex); Corsica (ex)

Family LEPORIDAE

Bunolagus monticularis (Thomas 1903)

\section{Riverine Rabbit}

South Africa

Caprolagus hispidus (Pearson 1839)

Hispid Hare, Assam Rabbit

Bangladesh (ex?) [602,603,604,970]; India [38]; Nepal [756]

Lepus flavigularis Wagner 1844

Tehuantepec Hare, Tehuantepec Jack Rabbit

Mexico [906]

Nesolagus netscheri (Schlegel 1880)

Sumatran Short-eared Rabbit, Sumatran Rabbit

Indonesia: Sumatra

Pentalagus furnessi (Stone 1900)

Ryukyu Rabbit

Japan: Ryukyu Islands

Romerolagus diazi (Diaz 1893)

Volcano Rabbit, Teporingo

Mexico [906]

Sylvilagus graysoni (J.A. Allen 1877)

Tres Marias Cottontail

Mexico: Tres Marias Islands [906]

Sylvilagus insonus (Nelson 1904)

Omilteme Cottontail

Mexico (ex) [906] 


\section{Order RODENTIA}

\section{Family APLODONTIDAE}

Aplodontia rufa nigra Taylor 1914

Id $\quad 656$

Point Arena Mountain Beaver

USA: California

Aplodontia rufa phaea Merriam 1899

Id $\quad 656$

Point Reyes Mountain Beaver

USA: California

Family SCIURIDAE

Cynomys mexicanus Merriam 1892

Mexican Prairie Dog

Mexico [906]

Cynomys parvidens J.A Allen 1905

Utah Prairie Dog

USA

Epixerus ebii (Temminck 1853)

$111 \mathrm{GH}$

724,941

Ebian's Palm Squirrel, Temminck's Giant Squirrel

Côte d'lvoire; Ghana; Liberia [628]; Sierra Leone

Glaucomys sabrinus coloratus Handley 1953

E

656

Carolina Flying Squirrel

USA

Glaucomys sabrinus fuscus Miller 1936

E

656

Virginia Flying Squirrel

USA

Glaucomys volans goldmani (Nelson 1904)

R 280

Mexican Fying SquirreI

Mexico [906]

Glaucomys volans guerreroensis Diersing 1980

R $\quad 280$

Guerrero Flying Squirrel

Mexico [906]

Glaucomys volans oaxacensis Goodwin 1961

Oaxaca Flying Squirrel

Mexico [906]

Marmota caudata (Geoffroy 1842-1843)

III IN

Long-tailed Marmot

Afghanistan; China; lndia; Kyrgyzstan; Pakistan; Tajikistan

Marmota himalayana (Hodgson 1841)

III IN

Himalayan Marmot

China; India; Nepal

Marmota menzbieri (Kashkarov 1925)

R

Menzbier's Marmot

$\mathrm{V}$

$27,106,712$

Kazakhstan [106]; Tajikistan [106]; Turkmenia [106]; Uzbekistan [106] 
Marmota vancouverensis Swarth 1911

- $\quad$ E $442,787,1098$

Vancouver Island Marmot

Canada: Vancouver Island

Ratufa affinis (Raffles 1821)

11

217,723

Cream-coloured Giant Squirrel

Brunei [119]; Indonesia [934,1009]; Malaysia: Peninsular Malaysia, Sabah [266], Sarawak; Myanmar; Singapore (ex?); Thailand $[96,151]$

Ratufa bicolor (Sparrman 1778)

11

514

Black Giant Squirrel

Bangladesh [602,604]; Bhutan [207,953]; Cambodia [260,837]; China [260,1169]; India [884]; Indonesia: Anambas Islands, Bali, Batu Islands, Java, Natuna Islands, Sumatra [934]; Lao P.D.R. [260,271,417]; Malaysia: Peninsular; Myanmar [955]; Nepal [756]; Thailand [151]; Viet Nam [260,837,853,1025]

Ratufa indica (Erxleben 1777)

(except subspecies dealbata, elphinstonei)

Indian Giant Squirrel

India

\section{Ratufa indica dealbata (Blanford 1897)}

Dangs Giant Squirrel

India

Ratufa indica elphinstoni (Sykes 1831)

Maharashtra Giant Squirrel

India

Ratufa macroura (Pennant 1769)

Grizzled Giant Squirrel

India [903]; Sri Lanka [865]

Sciurus deppei Peters 1863

Deppe's Squirrel

Belize; Costa Rica; EI Salvador [178]; Guatemala [404]; Honduras [703]; Mexico [906]; Nicaragua
$11 \quad$ - $\quad 99,100,884,1182$

$11 \quad \mathrm{E} \quad 514$

$11 \quad$ E $\quad 514$

$11 \quad-\quad 884$

III CR - $\quad 442,770$
Sciurus niger cinereus Linnaeus 1758

Delmarva Fox Squirrel, Bryant's Fox Squirrel

USA

Syntheosciurus brochus Bangs 1902

Mountain Squirrel

Costa Rica; Panama [316]

Tamias minimus atristriatus V.Bailey 1913

(= Eutamias minimus atristriatus)

New Mexico Least Chipmunk

USA: New Mexico

Tamias quadrivittatus australis Patterson 1980

(= Eutamias quadrivittatus australis)

Organ Mountains Chipmunk

USA: New Mexico

Tamiasciurus hudsonicus grahamensis (J.A. Allen 1894) Mount Graham Red Squirrel

USA: Arizona

\section{E $\quad 1098$}

K $\quad 514$

E $\quad 442$

E $\quad 846$ 


\section{Family GEOMYIDAE}

Thomomys umbrinus emotus Goldman 1933

R

Southern Pocket Gopher

USA: New Mexico

Zygogeomys trichopus Merriam 1895

Id

514

Michoacan Pocket Gopher

Mexico [906]

Family HETEROMYIDAE

Dipodomys elator Merriam 1894

Texas Kangaroo-rat

USA

Dipodomys gravipes Huey 1925

E $\quad 656$

San Quintin Kangaroo Rat

Mexico: Baja California [906]

Dipodomys heermanni morroensis (Merriam 1907)

E

Morro Bay Kangaroo-rat

USA

Dipodomys ingens (Merriam 1904)

ld $\quad 656$

Giant Kangaroo Rat

USA: California

Dipodomys microps leucotis Goldman 1931

K $\quad 656$

Houserock Chisel-toothed Kangaroo Rat

USA: Arizona [442]

Dipodomys nitratoides exilis Merriam 1894

Fresno Kangaroo Rat

USA: California [442]

Dipodomys nitratoides nitratoides Merriam 1894

Tipton Kangaroo Rat

USA: California

Dipodomys stephensi (Merriam 1907)

E $\quad 656$

Stephens' Kangaroo Rat

USA: California

Perognathus alticola Rhoads 1894

White-eared Pocket Mouse

USA: California

Perognathus inornatus psammophilus (von Bloeker 1937)

(= P. longimembris psammophilus)

Salinas Pocket Mouse

USA: California

Perognathus longimembris brevinasus Osgood 1900 
Family CASTORIDAE

Castor canadensis frondator Mearns 1897

$-\quad$ E $\quad 566$

Broad-tail Beaver

Mexico [906]

Castor canadensis mexicanus V. Bailey 1913

Rio Grande Beaver

Mexico [906]

Castor fiber birulai Serebrennikov 1929

Mongolian Beaver

Mongolia

\section{Family ANOMALURIDAE}

Anomalurus beecrofii Fraser 1853

11I GH

724

Beecroft's Flying Squirrel

Angola [531]; ?Benin; ?Burkina Faso [944]; Cameroon [941]; ?Central African Republic [689]; ?Chad [689]; Congo [941]; ?Côte d'Ivoire; Equatorial Guinea: Fernando Po [941]; Gabon [691]; ?Ghana; Guinea [937,941]; Guinea-Bissau; Liberia [14,628,941]; Nigeria [20]; Senegal; Sierra Leone [941]; ?Togo; Uganda [273]; Zaire [896,991]; Zambia

Anomalurus derbianus (Gray 1842) $\quad$ III GH $\quad-\quad 724$

Lord Derby's Flying Squirrel

Angola [941]; ?Benin; ?Burkina Faso; ?Burundi; Cameroon [941]; ?Central African Republic [689]; ?Chad [689]; Congo [691]; ?Côte d'Ivoire; Equatorial Guinea: Fernando Po [182,1093]; Gabon [941]; Ghana [941]; Guinea [937]; Kenya; Liberia [14,628]; Malawi [78]; Mozambique [1017]; Nigeria [941]; ?Rwanda; Sierra Leone [941,1086]; Tanzania: including Zanzibar [941]; ?Togo; Uganda [273]; Zaire [896]; Zambia

Anomalurus peli (Temminck 1845)

$111 \mathrm{GH}$ -

724

Pel's Flying Squirrel

?Burkina Faso [944]; Côte d'Ivoire [941]; Ghana [941]; Liberia [14,628,941]; Sierra Leone

Idiurus macrotis Miller 1898

III GH

724

Long-eared Flying Squirrel

?Benin; Cameroon [941]; ?Central African Republic; ?Congo; ?Côte d'lvoire; Gabon [691]; Ghana [941];

Liberia [628]; Nigeria; Sierra Leone [941]; ?Togo; Zaire [895,896,941]

Family CRICETIDAE

Neotoma anthonyi J.A Allen 1898

E $\quad 514$

Anthony's Woodrat

Mexico: Todos Santos Island [906]

Neotoma bunkeri Burt 1932

E $\quad 514$

Bunker's Woodrat

Mexico [906]

Neotoma floridana smalli Sherman 1955

E $\quad 656$

Key Lango Woodrat

USA [442]

Neotoma fuscipes riparia Hooper 1938

V $\quad 442$

San Joaquin Valley Woodrat

USA: California 
Neotoma martinensis Goldman 1905

San Martin Island Woodrat

Mexico: San Martin Island [906]

Oryzomys argenzalus Spitzer \& Lazell 1978

( $=0$. palustris argentatus)

Silver Rice Rat

USA: Florida

Oryzomys nelsoni Merriam 1898

Nelson's Rice Rat

Mexico (ex?) [906]

Peromyscus gossypinus allapaticola Schwartz 1962

Key Largo Cotton Mouse

USA

Peromyscus pembertoni Burt 1932

Ex $\quad 442$

Pemberton's Deer Mouse

Mexico: San Pedro Nolasco lsland (ex) [906]

Peromyscus polionotus allophrys Bowen 1968

E

656,1111

Choctawhatchee Beach Mouse

USA

Peromyscus polionotus ammobates Bowen 1968

E

656,1111

Alabama Beach Mouse

USA

Peromyscus polionotus niveiventris Chapman 1889

South-eastern Beach Mouse

USA [442]

Peromyscus polionotus phasma Bangs 1898

E $\quad 656$

Anastasia Island Beach Mouse

USA [442]

Peromyscus polionotus trissyllepsis Bowen 1968

E 656,1111

Perdido Key Beach Mouse

USA

Reithrodontomys megalotis limicola von Bloeker 1932

Southern Marsh Harvest Mouse

USA: California

Reithrodontomys raviventris Dixon 1908

E $\quad 442,999,1098$

Saltmarsh Harvest Mouse

USA: California

Sigmodon arizonae plenus (Goldman 1928)

E $\quad 656$

(=S. hispidus plenus)

Colorado River Cotton Rat

USA: ?Arizona, California [143] 
Family ARVICOLIDAE

Microtus breweri (Baird 1858)

R $\quad 442,1098$

(= M. pennsylvanicus breweri)

Beach Vole

USA: Massachusetts - Muskeget Island

Microtus californicus scirpensis V. Bailey 1918

E $\quad 656$

Amargosa Vole

USA: California [442]

Microtus mexicanus hualpaiensis Goldman 1938

Hualapai Vole

USA: Arizona

Microtus mexicanus navaho Benson 1934

USA: Arizona

Microtus pennsylvanicus chihuahuensis Bradley \& Cockrum 1968

Chihuahua Vole

Mexico [906]

Microtus pennsylvanicus dukecampbelli Woods, Post \& Kilpatrick 1982

Florida Saltmarsh Vole

E $656,920,1186$

USA: Florida

Pitymys bavaricus Konig 1962

Ex

Bavarian Pine Vole

Germany: West (ex)

Family MURIDAE

Batomys granti Thomas 1895

Id 483,656

Luzon Forest Rat

Philippines: Luzon

Conilurus albipes (Lichtenstein 1829)

Ex

$1044,1098,1149$

Rabbit-eared Tree-rat, White-footed Tree-rat

Australia (ex)

Leggadina lakedownensis Watts 1976

K $\quad 656,1143$

Lakeland Downs Mouse

Australia

Leporillus apicalis (Gould 1854)

Ex

$1044,1098,1149$

Lesser Stick-nest Rat, White-tipped Stick-nest Rat

Australia (ex)

Leporillus conditor (Sturt 1848)

I R

R $\quad 838,1044,1098,1149$

Greater Stick-nest Rat

Australia: Franklin Island

Macruromys elegans Stein 1933

K

Western Small-toothed Rat, Lesser Small-toothed Rat

Indonesia: Irian Jaya 
Melomys albidens Tate 1951

K $\quad 352$

White-toothed Melomys

Indonesia: Irian Jaya

Notomys amplus Brazenor 1936

Ex $\quad 1044,1098,1149$

Short-tailed Hopping-mouse

Australia (ex)

Notomys aquilo Thomas 1921

K

$838,1044,1098,1] 49$

(includes $N$. carpentarius Johnson 1959)

Northern Hopping-mouse

Australia

Notomys fuscus (W. Jones 1925)

V $\quad 1044,1149$

Dusky Hopping-mouse

Australia

Notomys longicaudatus (Gould 1844)

Ex $1044,1098,1149$

Long-tailed Hopping-mouse

Australia (ex)

Notomys macrotis Thomas 1921

Ex $1044,1098,1149$

(includes $N$. megalotis)

Big-eared Hopping-mouse

Australia (ex)

Notomys mordax Thomas 1922

Ex $1044,1098,1149$

Darling Downs Hopping-mouse

Australia (ex)

Pseudomys fieldi (Waite 1896)

Ex $1044,1098,1149$

Alice Springs Mouse

Australia (ex)

Pseudomys fumeus (Brazenor 1934)

R $735,838,1044,1149$

Smoky Mouse

Australia: Victoria

Pseudomys gouldii (Waterhouse 1839)

Ex 1143

Gould's Mouse

Australia (ex)

Pseudomys occidentalis Tate 1951

R $\quad 1143$

Western Mouse

Australia

Pseudomys oralis Thomas 1921

Hastings River Mouse

Australia

Pseudomys pilligaensis Fox \& Broscoe 1980

Id $\quad 1143$

Pilliga Mouse

Australia

Pseudomys praeconis Thomas 1910

R $\quad 529,1044,1098,1149$

Shark Bay Mouse

Australia 
Pseudomys shortridgei (Thomas 1907)

V $\quad 838,1044,1149$

Heath Rat, Shortridge's Native Mouse, Blunt-faced Rat

Australia: Victoria, W. Australia (ex)

Rattus giluwensis Hill 1960

R $\quad 352$

Giluwe Rat

Papua New Guinea

Solomys ponceleti (Troughton 1935)

Poncelet's Giant Rat

Papua New Guinea: Bougainville

Tokudaia osimensis muenninki Johnson 1946

E

27

North Okinawa Spiny Rat

Japan: North Okinawa 1sland

Xenuromys barbatus (Milne-Edwards 1900)

R

352

Rock-dwelling Rat, Mimic Tree-rat

Indonesia: Irian Jaya [352]; Papua New Guinea [352]

Xeromys myoides Thomas 1889

E $\quad 514$

False Water-rat, False Swamp Rat

Australia

Zyzomys pedunculatus (Waite 1896)

Central Rock-rat, Macdonnell Range Rock-rat

Australia

\section{Family ZAPODIDAE}

Zapus hudsonius luteus (Miller 1911)

E

773

(=Z. princeps luteus)

Meadow Jumping Mouse

USA: New Mexico

Zapus trinotatus orarius Preble 1899

Point Reyes Jumping Mouse

USA: California

\section{Family HYSTRICIDAE}

Hystrix cristata Linnaeus 1758

III GH

724

North African Crested Porcupine

[Albania]; Algeria [941]; ?Benin; Burkina Faso [944]; Cameroon [557]; Central African Republic [689]; Chad [689]; ?Côte d'Ivoire; Egypt (ex?) [835]; ?Equatorial Guinea; Ethiopia [245,1191]; ?Gabon; Gambia [941]; Ghana [941]; Greece; ?Guinea; Italy: including Sicily; Kenya [941]; Liberia [14,628]; Libya [528,941]; ?Mali; Mauritania; Morocco [183]; Niger [805,941]; Nigeria [1,940]; Senegal [941]; Sierra Leone [941, 1086]; Somalia [337,367]; Sudan [503]; Tanzania; ?Togo; Tunisia [941]; Turkey; Uganda [120,273]; Yugoslavia; Zaire

\section{Family ERETHIZONTIDAE}

Chaetomys subspinosus (Olfers 1818)

Id $\quad 968,1098$

Thin-spined Porcupine

Brazil 
Sphiggurus mexicanus (Kerr 1792)

III HN

(= Coendou mexicanus)

Mexican Tree Porcupine

?Belize; Costa Rica; El Salvador [178]; Guatemala [404]; Honduras [703]; Mexico [906]; ?Nicaragua; Panama [316]

Sphiggurus spinosus (F. Cuvier 1822)

11I UY

185

(= Coendou spinosus)

Spiny Tree Porcupine

Argentina [915]; Brazil; Paraguay [915]; Uruguay [915]

Family DINOMYIDAE

Dinomys branickii Peters 1873

E

Pacarana

Bolivia; Brazil; Colombia [316]; Ecuador; Peru; Venezuela [146,316]

Family AGOUTIDAE

Agouti paca (Linnaeus 1766)

1II HN -

514

(= Cuniculus paca)

Spotted Paca

Argentina: Misiones [915]; Belize; Bolivia [1198]; Brazil; Colombia; Costa Rica; [Cuba]; ?Ecuador; El Salvador [178]; French Guiana; Guatemala [404]; Guyana [316]; Honduras [703]; Mexico [906]; Nicaragua; Panama [316]; Paraguay [915]; ?Peru; Suriname [316]; Trinidad and Tobago [13]; Venezuela [316]

\section{Family DASYPROCTIDAE}

Dasyprocta punctata Gray 1842

III HN -

514

\section{Central American Agouti}

Argentina [699,915]; ?Belize; Bolivia [1198]; Brazil; [Cayman Islands]; Colombia [316]; Costa Rica; [Cuba]; Ecuador [316]; El Salvador [178]; ?French Guiana; Guatemala [404]; ?Guyana; Honduras [699]; Mexico [906]; Nicaragua; Panama [316]; Paraguay [915]; ?Peru; ?Suriname; Venezuela [316]

\section{Family CHINCHILLIDAE}

Chinchilla brevicaudata Waterhouse 1848

I Id $\quad 1098$

Short-tailed Chinchilla

Argentina [124,699,701,915]; Bolivia [1198]; Chile [394,746,915]; Peru (ex?) [851]

Chinchilla lanigera (Molina 1782)

I Id $394,746,915,1098$

Long-tailed Chinchilla

?Argentina [124]; Chile

\section{Family CAPROMY1DAE}

Capromys angelcabrerai Varona 1979

Cabrera's Hutia

Cuba: Cay Ana Maria

Capromys auritus Varona 1970

Large-eared Hutia

Cuba: Cay Fragoso

Capromys garridoi Varona 1970

Garrido's Hutia

Cuba: Canarreos Archipelago 
Capromys gundlachi Chapman 1901

Chapman's Prehensile-tailed Hutia

Cuba: lsle de Juventud

Capromys melanurus Poey 1865

Bushy-tailed Hutia

Cuba

Capromys meridionalis Varona 1986

ld $\quad 17,656$

(= Mysateles meridionalis)

Isla de la Juventud Tree Hutia

Cuba: Isle de Juventud [576]

Capromys nanus G.M. Allen 1917

Dwarf Hutia

Cuba

Capromys sanfelipensis Varona \& Garrido 1970

E $1098,1119,1120$

Little Earth Hutia, Land Hutia

Cuba (ex?)

Geocapromys brownii (Fischer 1830)

(= Capromys brownii)

(includes $G$. thoracatus)

Jamaican Hutia, Indian Coney, Brown's Hutia

Honduras: Little Swan Island (ex) [703]; Jamaica

Geocapromys ingrahami (J.A. Allen 1891)

R $\quad 1098$

(= Capromys ingrahami)

Bahamian Hutia, Ingraham's Hutia

Bahamas: Plana Keys

Plagiodontia aedium F. Cuvier 1836

(includes $P$. hylaeum Miller 1929)

Hispaniolan Hutia, Cuvier's Hutia

Dominican Republic; Haiti

Family OCTODONTIDAE

Octomys barrerae Lawrence 1941

(= Tympanoctomys barrerae)

Red Vizcacha Rat

Argentina

\section{Order CETACEA}

\section{Family PLATANISTIDAE}

Inia geoffrensis (de Blainville 1817)

Amazon River Dolphin, Boutu

(Found in all major rivers of the Orinoco and Amazon systems)

Bolivia [1198]; Brazil; Colombia [316]; Ecuador; Peru; Venezuela [316] 
Lipotes vexillifer Miller 1918

I $\quad E$

$27,36,446,620,753,1147,1203$

Whitefin Dolphin, White Flag Dolphin, Changjiang Dolphin

(Only found in the Chiangjiang River of China, may occasionally enter other rivers during times of flood)

China: Tung Ting Lake, Changjiang and Tsien Tang Rivers

$\begin{array}{llll}\text { Platanista gangetica (Roxburgh 1801) I } & \text { V } & 36,620,753,1147\end{array}$

Ganges River Dolphin, Ganges Susu

(Ganges and Brahmaputra river systems)

Bangladesh [602,603,604,970]; ?Bhutan; India [38]; Nepal [756,867]

Platanista minor Owen 1853

I E

$27,36,133,620,1147$

(= P. indi Blyth $1859, P$. gangetica minor)

Indus Dolphin, Susu

(Indus River)

?India; Pakistan [930]

Pontoporia blainvillei (Gervais \& d'Orbigny 1844) II $\quad$ K $\quad 36,185,620,753,1147$

La Plata River Dolphin, Franciscana

(La Plata estuary and adjacent rivers and coasts)

Argentina [124,915]; Brazil; Uruguay [915]

Family ZIPHIIDAE

Berardius arnuxii Duvernoy 1851

I

K $\quad 36,185,620,1147$

Arnoux's Beaked Whale, Southern Four-toothed Whale

(Recorded infrequently in the Southern Ocean)

Argentina [124,915]; Australia; Chile [915]; Falkland 1slands [915]; New Zealand; South Africa [109,1020]

Berardius bairdii Stejneger $1883 \quad$ l $\quad$ K $\quad 36,620,1147$

Baird's Beaked Whale, Northern Four-toothed Whale, Giant Bottle-nosed Whale

(Apparently confined to deep waters of Pacific, from Japan and Califormia to Bering Sea)

Canada [442]; Japan; D.P.R. Korea [1184]; Korea Republic [1184]; Russia [753,1147]; USA [442]

Hyperoodon ampullatus (Forster 1770) $\quad$ I $\quad$ K $\quad 27,36,620,1147$

(= H. rostratus P.L.S. Müller 1776)

Northern Bottlenose Whale, Bottlehead

(North Atlantic, from New York and Azores to Svalbard, possibly including Mediterranean and as far south as Cape Verde)

Canada [442]; Cape Verde Islands; ?Denmark; Estonia [106]; Faeroe Islands (Denmark); France; Germany; Greenland; Iceland; Ireland; Latvia [106]; Lithuania [106]; Netherlands; Norway; Poland; Portugal; Russia [106]; Svalbard and Jan Mayen Islands; Sweden; United Kingdom; USA [442]
Hyperoodon planifrons Flower 1882
I
$\mathrm{K}$
$36,185,620,724,1147$

Southern Bottlenose Whale

(Circumpolar in Southern Hemisphere from Antarctic to Argentina, South Africa and Australia)

Argentina [109,124,915]; Australia; Brazil; Chile [915]; Falkland Islands; New Zealand; South Africa [1020]; Sri Lanka [437]; Uruguay [915]

Indopacetus pacificus (Longman 1926)

II K

$620,724,1147$

(= Mesoplodon pacificus)

(Probable IndoPacific distribution, known only from 2 skulls found on beaches in the Indian and South Pacific Oceans)

Indo-pacific Beaked Whale, Longman's Beaked Whale

Australia; ?Seychelles; Somalia 
Mesoplodon bidens (Sowerby 1804)

II

$\mathrm{K}$

620,1147

Sowerby's Beaked Whale

(Mainly North Sea and North Atlantic but few records from North America, possibly Mediterranean)

Belgium; Canada [442]; Denmark; France; Germany; 1reland; Italy; Netherlands; Norway; Poland; Sweden;

United Kingdom; USA [442]

Mesoplodon bowdoini Andrews 1908

11

K

620,1147

Splaytooth Beaked Whale, Andrews' Beaked Whale

(Temperate South Pacific)

Australia; ?French Southern and Antarctic Territories; New Zealand

Mesoplodon carlhubbsi Moore 1963

II $\quad \mathrm{K}$

620,1147

Arch-beaked Whale, Hubbs' Beaked Whale

(Temperate North Pacific from Japan and Oregon to Bering Sea)

Japan; New Zealand; USA [442]

Mesoplodon densirostris (Blainville 1817)

ll $\quad \mathrm{K}$

$620,724,1147$

Blainville's Beaked Whale

(Circum-equatorial; temperate and tropical waters)

Australia; Bahamas [442]; Canada [442]; Japan; Mauritius; Portugal; Seychelles; South Africa [1020]; Spain;

Taiwan; USA [442]

Mesoplodon europaeus (Gervais 1855) $\quad$ II $\quad$ K $\quad 185,620,1147$

Gervais' Beaked Whale, Gulf Stream Beaked Whale

(Caribbean and North-west Atlantic; single specimens from English Channel)

Cuba [442]; Jamaica [442]; Trinidad and Tobago [316]; United Kingdom; USA [442]

Mesoplodon ginkgodens Nishiwaki \& Kamiya 1958 II $\quad$ K $\quad 36,620,1147$

Ginkgo-toothed Beaked Whale

(Tropical Pacific)

Japan; Sri Lanka [437]; ?Taiwan; USA [442]

Mesoplodon grayi Von Haast 1876

lI $\quad \mathrm{K}$

$36,185,620,1147$

Gray's Beaked Whale, Southern Beaked Whale

(Circumpolar in Southern Hemisphere, south of $30^{\circ} \mathrm{S}$, but mainly around New Zealand)

Argentina [915]; Australia; Chile [915]; Netherlands; New Zealand; South Africa [1020]

Mesoplodon hectori (Gray 1871)

11

K

$36,185,620,1147$

Hector's Beaked Whale, Skew-heaked Whale

(Circumpolar in Southern Hemisphere)

Argentina [124,915]; Australia; Chile [915]; Falkland Islands [915]; New Zealand; South Africa [1020]; USA [719]

Mesoplodon layardii (Gray 1865)

II

K $\quad 620,1147$

Layard's Beaked Whale, Strap-toothed Whale

(Pacific and Indian Oceans, south of $30^{\circ} \mathrm{S}$; sightings in South Africa and Falkland Islands)

Argentina [915]; Australia; Chile [915,1124]; Falkland Islands; New Zealand; South Africa [1020]; Uruguay [915]

Mesoplodon mirus True 1913

II $\quad \mathrm{K}$

$36,620,1147$

True's Beaked Whale

(North Atlantic to United Kingdom; few records from South Africa)

Canada [442]; Ireland; South Africa [1020]; United Kingdom; USA [442] 
Shepherd's Beaked Whale, Tasman Whale

(Temperate South Pacific and West Atlantic)

Argentina [124,915]; Australia; Chile [915]; New Zealand

Ziphius cavirostris G. Cuvier 1823

Cuvier's Beaked Whale, Goose-beaked Whale

(Widespread in all major oceans, but rarely found in polar regions; most records from North Atlantic and Mediterranean)

Argentina [124,915]; Australia; Bahamas [442]; Barbados [187]; Brazil; Canada [442]; Chile [915]; Cuba [442]; Galapagos Islands; France; Indonesia: Java; Ireland; Italy; Japan; Mexico; Morocco; Namibia [1020]; Netherlands Antilles: Bonaire, Curacao [316]; New Zealand [364]; Panama; Russia [1147]; Saint Vincent; South Africa [1020]; Spain; Sri Lanka [437]; Sweden; United Kingdom; USA [442]; Uruguay [915]; Venezuela

\section{Family PHYSETERIDAE}

Kogia breviceps (Blainville 1838)

$11 \mathrm{~K}$ $36,185,620,723,753,1147$

Pygmy Sperm Whale

(Warm waters, mainly South-east Pacific, west Indian Ocean, Australia, south-east USA)

Argentina [915]; Australia; Azores; Canada [442]; Cuba [442]; France; Hong Kong; India; Indonesia; Japan; Malaysia: Sarawak; Mozambique; Namibia [1020]; Netherlands; New Zealand; Pakistan [930]; Peru; Senegal; South Africa [1020]; Sri Lanka [437]; United Kingdom; USA [442]; Uruguay [915]
Kogia simus (Owen 1866)
11
$\mathbf{K}$
$36,620,724,753,1147$

Dwarf Sperm Whale

(Warm waters, mainly off Japan and eastern USA, but also Indian Ocean)

Australia; Canada; Chile [915]; Cuba [442]; India; Japan; Mexico [442]; Mozambique; Oman; Saint Vincent [442]; South Africa [1020]; Sri Lanka [437]; USA [442]

$\begin{array}{lllll}\text { Physeter macrocephalus Linnaeus } 1766 & 1 & \text { K } & 36,379,620,1147\end{array}$

( $=$ P. catodon Linnaeus 1766)

Sperm Whale, Cachelot

(Found in all major oceans, from Arctic to Antarctic, including semi land-locked seas such as Mediterranean) Argentina [109,915]; Australia; Azores; Belgium; Brazil; Canada [442]; Chile [915]; China; Colombia [316]; Costa Rica [555]; Falkland 1slands [915]; French Polynesia; Greenland; India [38]; Indonesia: Java, Sumatra [934]; Ireland; Japan; D.P.R. Korea [1184]; Korea Republic [1184]; Liberia [14]; Madeira; Mexico [847]; Mozambique [1017]; Myanmar; Netherlands [176]; New Zealand; Norway; Panama [442]; Saint Helena; South Africa [1020]; Spain; Sri Lanka [437]; Suriname; Thailand [151]; United Kingdom; USA [442], Uruguay [915]; Venezuela [316]

\section{Family MONODONTIDAE}

$\begin{array}{llll}\text { Delphinapterus leucas (Pallas 1776) } & \text { II } & \text { K } & 36,620,753,1147\end{array}$

\section{White Whale, Beluga}

(Circumpolar in Arctic and sub-Arctic with scattered records to France and Japan; often in estuaries)

Belgium; Canada [442]; Denmark; Finland; France; Germany; Greenland; Japan; Norway; Poland; Russia [106,126]; Sweden; Svalbard and Jan Mayen Islands; United Kingdom; USA [442] 
Monodon monoceros Linnaeus 1758

11

$\mathrm{K}$

$36,620,753,919,1147$

Narwhal, Unicorn Whale

(Circumpolar and virtually confined to limits of Arctic pack ice; vagrants as far south as the United Kingdom; Canada [442]; Germany; Greenland; Iceland; Netherlands; Norway; Russia [106,760]; Svalbard and Jan Mayen Islands; United Kingdom; USA [442]

\section{Family DELPHINIDAE}

Cephalorhynchus commersonii (Lacépède 1804)

Il $\quad \mathrm{K}$

K $\quad 620,753,1147$

Commerson's Dolphin, Piehald Dolphin

(Temperate South Atlantic and Indian Oceans)

Argentina [124,915]; Chile [915]; Falkland lslands; French Southern and Antarctic Territories: Kerguelen Islands [931]; Saint Helena

Cephalorhynchus eutropia Gray 1846

11

K $\quad 36,620,915,1147$

Black Dolphin, Chilean Dolphin, White-bellied Dolphin

(Cold, inshore waters of Chile, between $33^{\circ} \mathrm{S}$ and $55^{\circ} \mathrm{S}$ )

Chile

Cephalorhynchus heavisidii (Gray 1828)

11

K $\quad 620,724,753,1147$

Heaviside's Dolphin, Benguela Dolphin

(Cold, inshore waters of Benguela current, South Atlantic)

Namibia; South Africa [1020]

Cephalorhynchus hectori (van Beneden 1881)

$11 \quad$ Id

$36,620,723,753,1147$

White-headed Dolphin, Hector's Dolphin, New Zealand Dolphin

(South-west and West Pacific Ocean)

Malaysia: Sarawak; New Zealand; Viet Nam [1025]

Delphinus delphis Linnaeus 1758

II K

$36,620,723,724,753,1147$

Common Dolphin, Atlantic Dolphin, Pacific Dolphin, Saddle-backed Dolphin

(Recorded from all warm temperate and tropical seas, including the Mediterranean and Black Seas)

Algeria; Argentina [915]; Australia; Bangladesh [604]; Bulgaria; Canada [442]; Chile [915]; Costa Rica [442]; Côte d'Ivoire; Egypt; France; Georgia [679]; Greenland; Honduras [703]; Hong Kong; lceland; India [38]; Indonesia [934]; Israel; Japan; D.P.R. Korea [1184]; Korea Republic [1184]; Libya [528]; Madagascar; Malaysia: Sarawak; Malta; Mauritania; Morocco; Mozambique [1017]; Namibia [1020]; Netherlands; New Zealand; Norway; Pakistan; Panama; Poland; Portugal; Romania; Russia [679]; Senegal; Somalia; South Africa [1020]; Spain; Sri Lanka [437]; Thailand [151]; Tunisia; Turkey [629]; Ukraine [679]; United Kingdom; USA [442]; Uruguay [915]; Venezuela [316]

Delphinus tropicalis van Bree 1971

11

K $\quad 514$

$(=D$. delphis tropicalis $)$

Tropical Dolphin

(Arabian Sea to South China Sea)

India; Pakistan [930]; Somalia

Feresa attenuata Gray 1875

Il K

$36,620,724,753,1147$

(=F. occulta Jones \& Packard 1956)

Pygmy Killer Whale, Slender Blackfish

(Recorded sporadically from warm temperate and tropical waters, including North and South Atlantic, lndian Ocean and North Pacific)

Australia; Costa Rica; Japan; Mexico; Namibia [1020]; Saint Vincent [442]; Senegal; South Africa [1020]; Sri Lanka [437]; USA [442]; Viet Nam [437] 
(= G. sieboldii Gray 1846)

Short-finned Pilot Whale, Pacific Pilot Whale

(Warm temperate and tropical waters, including Atlantic, Pacific and Indian Oceans)

Australia; Bangladesh [604]; Guam; Guatemala [442]; India; Indonesia [217]; Japan; Myanmar; Saint Vincent [442]; Senegal; South Africa [1020]; Sri Lanka [437]; USA [442]; Venezuela
Globicephala melaena (Traill 1809)
Il $\mathrm{K}$
$36,620,723,753,1147$

(includes G. edwardii (Smith 1834))

\section{Long-finned Pilot Whale}

(Occurs in two separate populations, the southem being circumpolar in cool temperate waters of Atlantic, Pacific and Indian Oceans north of the Antarctic Convergence; the North Atlantic population extending from USA and the Mediterranean to the Barents Sea. Apparently absent from Nortb Pacific)

Argentina [109,915]; Australia; Canada [442]; Chile [915]; Denmark; Faeroe Islands ; France; Germany; Greenland [442]; Jceland; Ireland; Italy; Malaysia: Sarawak; Malta; Netherlands; New Zealand; Norway; Peru; South Africa [1020]; Spain; Sweden; United Kingdom; USA [442]; Uruguay [915]
Grampus griseus (G. Cuvier 1812)
II $\mathrm{K}$
$36,620,723,724,745,753,1147$

Risso's Dolphin, Grey Dolphin

(Worldwide distribution in tropical and warm temperate waters)

Argentina [124,915]; Australia; Bahamas [442]; Canada [442]; Chile [915]; China; Costa Rica [555]; Cuba [442]; France; Georgia [679]; Indonesia; Ireland; Italy; Japan; Malaysia: Sarawak; Malta; Mexico [648]; Namibia [1020]; Netherlands; New Zealand; Russia [679]; Saint Vincent [442]; Solomon Islands; South Africa [1020]; Spain; Sweden; United Kingdom; Ukraine [679]; USA [442]
Lagenodelphis hosei Fraser 1956
II $\quad \mathrm{K}$
$36,620,723,1147$

Fraser's Dolphin, Sarawak Dolphin

(Reported from widely scattered localities in tropical Pacific and Atlantic regions)

Australia; Costa Rica: Cocos Island; French Polynesia; Japan; Kiribati; Malaysia: Sarawak; Philippines; Saint Vincent; South Africa [1020]; Sri Lanka [437]; Taiwan
Lagenorhynchus acurus (Gray 1828)
II
K
$620,753,1147$

Atlantic White-sided Dolphin

(North Atlantic from the USA to Norway, including the Mediterranean and Baltic Seas)

Canada [442]; Denmark; Faeroe Islands ; France; Greenland; Ireland; Netherlands; Norway; Portugal; Russia [753]; Sweden; United Kingdom; USA [442]

\section{Lagenorhynchus albirostris (Gray 1846) \\ II \\ $\mathbf{K}$ \\ $36,620,753,1147$}

White-beaked Dolphin

(North Atlantic and western Arctic from the USA and Spain to Svalbard, including the Baltic, Davis Strait and Barents Sea)

Belgium; Canada [442]; Denmark; Faeroe Islands ; Germany; Greenland [442]; Iceland; Ireland; Netherlands; Norway; Poland; Portugal; Russia [753]; ?Spain; Sweden; Turkey [629]; United Kingdom

Lagenorhynchus australis (Peale 1848)

II $\quad \mathrm{K} \quad 185,620,753,1147$

Peale's Dolphin, Blackchin Dolphin

(Cold, coastal waters of southern South America)

Argentina [915]; Chile [915]; Falkland Islands [915]

$\begin{array}{lllll}\text { Lagenorhynchus cruciger (Quoy \& Gaimard 1824) } & \text { II } & \text { K } & 620,1147\end{array}$

Hourglass Dolphin

(Circumpolar, recorded from high southern latitudes to the edge of the pack ice)

Argentina [109,915]; Chile [915]; Falkland Islands 
Lagenorhynchus obliquidens Gill 1865

11

K

$620,753,1147$

Pacific White-sided Dolphin

(Offshore in cooler regions of the North Pacific)

Japan; D.P.R. Korea [1184]; Korea Republic [1184]; Mexico [442]; Russia [753]; USA [442]

Lagenorhynchus obscurus (Gray 1828)

IJ $\quad \mathrm{K}$

$185,620,724,753,1147$

(includes L. superciliosus (Lesson \& Gamot 1826))

Dusky Dolphin

(Circumpolar between about $30^{\circ} \mathrm{S}$ and $60^{\circ} \mathrm{S}$ )

Argentina [109,915]; Australia; Chile [915]; French Southern and Antarctic Territories; Madagascar; Namibia; New Zealand; Peru; South Africa [1020]

Lissodelphis borealis (Peale 1848)

II

$36,620,1147$

Northern Right Whale Dolphin

(Offshore in North Pacific from Japan and California to the Bering Sea)

Canada; Japan; Russia [1147]; USA [442]

Lissodelphis peronii (Lacépède 1804)

Il K $\quad 36,185,620,1147$

Southern Right Whale Dolphin

(Circumpolar in Southern Hemisphere, extending to $19^{\circ} \mathrm{S}$ off Chile and to Indonesia)

Argentina [109,9 I5]; Australia; British Antarctic Territory: South Shetland 1slands [403]; Chile [915]; Indonesia;

New Zealand; Papua New Guinea; South Africa [1020]

Orcaella brevirostris (Gray 1866)

11

K

$36,620,723,753,1147$

Irrawaddy Dolphin, Snubfin Dolphin

(Coastal waters from Bay of Bengal to South-east Asia and Australia, including major rivers - Ganges, Brahmaputra and Irrawaddy)

Australia; Bangladesh [604]; Brunei; Cambodia; India; Indonesia: Java [217], Kalimantan, Sumatra [934]; Lao P.D.R. [417]; Malaysia: Sabah [468], Sarawak; Myanmar; Papua New Guinea; Singapore; Thailand [151]; Viet Nam [1025]

Orcinus orca (Linnaeus 1758)

II $\quad$ K

$36,128,620,723,724,753,1147$

(includes 0 . glacialis Berzin \& Vladimirov 1983)

Killer Whale, Orca

(Reported from all sea areas, especially in cooler regions; extending well into the pack ice zone)

Argentina [915]; Australia; Bahamas [442]; Canada [442]; Chile [915]; Côte d'Ivoire; Denmark; Ecuador; Faeroe Islands ; France; Germany; Greenland [442]; Guam; Iceland; India; Indonesia; 1reland; Japan; D.P.R. Korea [1184]; Korea Republic [1184]; Madagascar; Malaysia: Sabah [468], Sarawak [217]; Mauritania; Mexico [442]; Morocco; Mozambique [1017]; New Zealand; Norway; Russia [753]; Saint Vincent [442]; Senegal; Seychelles; South Africa [1020]; Spain; Sri Lanka [437]; United Kingdom; USA [442]; Uruguay [915]; Viet Nam [1025]
Peponocephala electra (Gray 1846)
Il K
$620,753,1147$

Melon-headed Whale

(Widespread scattered records from warm temperate and tropical seas)

Australia; Bangladesh [604]; Costa Rica [555]; French Polynesia; Guam; Guatemala; India; Indonesia; Japan; Malaysia; Mexico; Pakistan [930]; Papua New Guinea; Saint Vincent [316]; Senegal; South Africa [130,1020]; Sri Lanka [437]; USA; Viet Nam [1025]

Pseudorca crassidens (Owen 1846)

$11 \quad \mathrm{~K} \quad 36,185,620,724,1147$

False Killer Whale

(Widespread, mainly in warm waters but strandings as far north as the Baltic)

Argentina [915]; Australia; Brazil; Canada [97]; Chile [915]; China; Cuba [442]; Denmark; Germany; India; Iraq [687]; Japan; Malta; Mexico [442]; New Zealand; Oman; Peru; Russia [1147]; Saint Helena; Saint Vincent [442]; South Africa [1020]; Spain; Sri Lanka [437]; Sweden; Tanzania; United Kingdom; USA [442]; Uruguay [915]; Venezuela [316] 
Sotalia fluviatilis (Gervais 1853)

$1 \mathrm{~K}$

$36,620,1147$

(includes S. brasiliensis (van Bẻnéden 1875), S. guianensis (van Bénéden 1864))

Tucuxi, Estuarine Dolphin, Guianian River Dolphin

(All major rivers in the Amazon system, extending to Central America)

Belize; Bolivia [1198]; Brazil; Colombia; Costa Rica; French Guiana; Guatemala; Guyana; Honduras; Mexico; Panama; Peru; Suriname; Venezuela

$\begin{array}{llll}\text { Sousa chinensis (Osbeck 1765) } & \text { I } & K & 36,620,723,753,1147\end{array}$

(includes S. borneensis (Lydekker 1901), S. lentiginosa (Owen 1865), S. plumbea (Cuvier 1829))

Indopacific Humphack Dolphin

(Indian Ocean, including Red Sea, Arabian Gulf and Bay of Bengal, to South-east Asian region, south China coast and Changiiang, Min and Yu Rivers)

Australia; Bahrain; Bangladesh; Brunei; Cambodia; China; Egypt; Ethiopia; India; Indonesia [934]; Iran [646]; Iraq [687]; Israel; Kenya; Kuwait; Madagascar; Malaysia: Sarawak [217]; Mozambique [1017]; Myanmar; Oman; Pakistan [930]; Papua New Guinea; Qatar; Saudi Arabia; Somalia; South Africa [1020]; Sri Lanka [437]; Sudan; Tanzania; Thailand [151]; United Arab Emirates; Viet Nam; Yemen

Sousa teuszii (Kukenthal 1892)

I K $\quad 36,620,724,1147$

Atlantic Humpback Dolphin

(West coast of Africa, from Angola to Mauritania; enters rivers)

Angola; Benin; Cameroon; Congo; Côte d'Ivoire; Gabon; Gambia; Ghana; Guinea; Guinea-Bissau; Liberia; Mauritania; Nigeria; Senegal; Sierra Leone; Togo

Stenella attenuata (Gray 1846)

$11 \quad \mathrm{~K} \quad 620,724,753,1147$

$(=S$. dubia attenuata)

(includes S. dubia (Cuvier 1812), S. graffimani (Lönnberg 1934), S. malayana (Lesson 1826))

Pantropical Spotted Dolphin, Narrow-snouted Dolphin, Bridled Dolphin

(Widespread in warm deep waters)

Argentina [915]; Australia; Bangladesh; Cape Verde Islands; Costa Rica [555]; Honduras [703]; Indonesia; Japan; D.P.R. Korea [1184]; Korea Republic [1184]; Malaysia; Mexico [442]; Myanmar; New Zealand; Panama [442]; Peru; Seychelles; Singapore; Solomon Islands; South Africa [1020]; Sri Lanka [437]; Thailand [151]; USA [442]

Stenella clymene (Gray 1850)

II K

$620,857,1147$

Helmet Dolphin, Atlantic Spinner Dolphin, Clymene Dolphin

(Warm and tropical waters of North Atlantic)

Honduras [703]; USA

Stenella coeruleoalba (Meyen 1833)

II K

$36,620,753,1147$

(includes $S$. styx (Gray 1846))

Striped Dolphin, Euphrosyne Dolphin

(Widespread in tropical and temperate waters, extending to Greenland and possibly Bering Sea)

Argentina [915]; Australia; Belgium; Canada [442]; Costa Rica [555]; France; Greenland; Honduras [703]; Indonesia; Jamaica [442]; Japan; Mozambique [1020]; Panama; Seychelles; South Africa [1020]; Spain; Sri Lanka [437]; United Kingdom; USA [442]; Uruguay [915]

Stenella frontalis (Cuvier 1829)

II K

$620,724,1147$

(= S. plagiodon (Cope 1866), S. attenuata frontalis)

Atlantic Spotted Dolphin

(Offshore in Atlantic from USA to west Africa)

Algeria; Bahamas; Brazil; Cape Verde 1slands.; Gabon; Honduras [703]; Panama; Saint Helena; ?Senegal; USA 
Stenella longirostris (Gray 1828)

II

K

$185,620,1147$

(includes S. microps (Gray 1846), S. roseiventris (Wagner 1853))

Long-snouted Dolphin, Long-beaked Dolphin, Spinner Dolphin

(Widespread in warm waters)

Argentina [915]; Australia; Bangladesh [604]; Chile [915]; Costa Rica [555]; Galapagos Islands; French Polynesia; Honduras [703]; India; Indonesia; Japan; Mexico [442]; Papua New Guinea; Panama; Senegal; Singapore; Solomon 1slands; Sri Lanka [437]; USA [442]
Steno bredanensis (Lesson 1828)
II
K
$36,620,1147$

Rough-toothed Dolphin

(Widespread in warm temperate ocean areas, including Red Sea, Caribbean, Mediterranean and North Seas)

Argentina; Bangladesh; Brazil; Côte d']voire; Cuba; Ecuador; France; Honduras [703]; India; Indonesia [217]; Italy; Japan; Mexico; Mozambique [1017]; Netherlands; Portugal; Saint Helena; Saint Vincent; Senegal; South Africa; Sri Lanka [437]; Thailand [151]; USA [442]; Yemen

Tursiops truncatus (Montagu 1821) II $\quad$ K $\quad 36,185,620,723,753,1147$

(includes T. aduncus (Ehrenberg 1832), T. gilli (Dall 1873), T. nuuanu (Andrews 1911))

Bottle-nosed Dolphin

(Known from all temperate and tropical marine areas)

Argentina [915]; Australia [647]; Bangladesh; Barbados [442]; Bulgaria; Canada [442]; Chile [915]; China; Denmark; Finland; France; Georgia [679]; Germany; Greenland; Honduras [703]; India; Indonesia; 1reland; Italy; Japan; Libya [528]; Malaysia: Sarawak; Malta; Mexico [442]; Mozambique [1020]; Namibia [1020]; Netherlands; Pakistan [930]; Poland; Portugal; Puerto Rico [442]; Romania; Russia [679]; Saint Helena; Saint Vincent [442]; Senegal; Seychelles; South Africa [1020]; Sri Lanka [437]; Sweden; Taiwan; Thailand [151]; Tunisia; Turkey [629]; Ukraine [679]; United Kingdom; USA [442]; Uruguay [915]; Venezuela; Western Samoa [1113]

\section{Family PHOCOENIDAE}
Neophocaena phocaenoides
(G. Cuvier 1829)
I K
$36,620,721,723,753,1147$

Finless Porpoise

(Coastal and freshwater Indopacific regions from China and Japan to the Gulf of Arabia, including South-East Asian archipelago)

Bahrain; Bangladesh [604]; Brunei; Cambodia; China; Hong Kong; India; Indonesia [934]; Iran; 1raq; Japan; Myanmar; D.P.R. Korea; Korea Republic; Kuwait; Malaysia: Sabah [468], Sarawak [217]; ?New Zealand; Oman; Pakistan [930]; Philippines; Qatar; Saudi Arabia; Singapore; ?South Africa; Sri Lanka [437]; Taiwan; Thailand [151]; Viet Nam [1025]

Phocoena dioptrica Lahille 1912

II $\quad \mathrm{K} \quad 36,171,185,620,1147$

(= Australophocaena dioptrica)

Spectacled Porpoise

(Coastal waters of South-west Atlantic)

Argentina [915]; Chile [915]; Falkland Islands: including South Georgia [915]; New Zealand [98]; Uruguay [915]

Phocoena phocoena (Linnaeus 1758) II $\quad$ K $\quad 36,380,620,753,1147$

(includes $P$. vomerina Gill 1865)

Common Porpoise, Harbour Porpoise

(Coastal waters of North Pacific, North Atlantic and Arctic Oceans, from Japan and California to Chukchi Sea, and from Baffin Island to west Africa, including Baltic, Mediterranean and Black Seas)

Canada [442]; Denmark; France; Germany [139]; Georgia [679]; Greenland [442]; Iceland; 1reland; Japan; Mexico; Morocco; Netherlands; Norway; Poland; Russia [679]; Senegal; Spain; Turkey [629]; Ukraine [679]; United Kingdom; USA [442] 
Phocoena sinus Norris \& McFarland 1958

$$
1
$$

$\mathbf{E}$

$27,56,172,620,753,1147$

Vaquita, Cochito, Gulf Porpoise

(Northern half of Gulf of California)

Mexico [442]

Phocoena spinipinnis Burmeister 1865

II

K

$27,36,173,620,753,1147$

Burmeister's Porpoise, Black Porpoise

(Coastal South America)

Argentina [124,915]; Chile [915]; Peru; Uruguay [915]

Phocoenoides dalli (True 1885)

11

K

$36,620,753,1147$

Dall's Porpoise, White-flanked Porpoise

(North Pacific from Japan and Mexico to Bering Sea)

Canada [442]; China; Japan; D.P.R. Korea [1184]; Korea Republic [1184]; Mexico [442]; Russia [1147]; USA [442]

\section{Family ESCHRICHTIDAE}

Eschrichtius robustus (Lilljeborg 1861)

I $\quad$ - $\quad 36,620,921,1147$

(=E. gibbosus (Erxleben 1777 invalid))

Grey Whale, Devil Fish

(North Pacific from Korea and Oregon to Bering Sea; extinct in North Atlantic)

Canada [442]; China; Guadeloupe; Japan; D.P.R. Korea [1184]; Korea Republic [1 184]; Mexico [442]; Russia [1147]; USA [442]

\section{Family BALAENOPTERIDAE}

Balaenoptera acutorostrata Lacépède 1804

Minke Whale, Lesser Rorqual, Little Piked Whale

Widespread in all oceans but rarer in the tropics; extends into polar pack ice; recorded from Baltic and Black Seas)
Appendix I populations
1
K
$36,1147,113,210,620$

Argentina [109,124,915]; Australia; Bahamas [442]; Bangladesh; Brazil; Canada [442]; Chile; Colombia; Denmark; Ecuador; France; French Polynesia; Germany; Greenland: except west coast; Iceland; Ireland; India; Indonesia; Japan; D.P.R. Korea [1184]; Korea Republic [1184]; Malaysia; Mexico [442]; Myanmar; Netherlands; New Zealand; Norway; Peru; Russia [753]; South Africa [1020]; Spain; Sri Lanka [437]; Svalbard and Jan Mayen Islands; Sweden; Thailand [151]; United Kingdom; USA [442], Uruguay [915]

Appendix II populations

Greenland: west

\section{Balaenoptera borealis Lesson 1828}

Sei Whale

(Widespread from sub-polar waters to the equator; main Southern Hemisphere stock summers in South Atlantic and Scotia Sea)

Argentina [109,124,915]; Australia; Canada [442]; Chile [915]; Cuba [442]; Falkland Islands [915]; lceland; India; Indonesia [217]; Japan; D.P.R. Korea; Korea Republic; Malaysia: Sarawak; Mexico [442]; Mozambique; Norway; Poland; Réunion; Russia [1147]; South Africa [1020]; Spain; Suriname; Taiwan; Thailand [217]; United Kingdom; USA [442]; Uruguay [915]

\section{Balaenoptera edeni Anderson 1878}

Bryde's Whale, Tropical Whale

(Circum-equatorial in warm waters, mainly coastal: Pacific from Chile to California and from Hawaii to north-east Japan; Atlantic from Brazil to Chesapeake Bay, USA, including Gulf of Mexico and from South Africa to West Africa; Indian Ocean from South Africa to Western Australia, extending to the Seychelles, Bay of Bengal, Sumatra) 
Angola; Argentina [124]; Australia; Brazil; Chile [915]; Fiji; French Polynesia; Grenada [442]; Indonesia; Iraq [687]; Japan; Madagascar; Malaysia: Peninsular Malaysia, Sarawak; Mexico [442]; Mozambique; New Zealand; Pakistan; Peru; Saudi Arabia [800]; Senegal; Seychelles; Solomon Islands; South Africa; Sri Lanka [437], Taiwan; USA
Balaenoptera musculus (Linnaeus 1758)
1
E
$27,36,620,723,1147$

Blue Whale, Sulphur-bottom Whale

(Occurs in all major oceans, especially cold and temperate waters, but recorded infrequently from tropical seas and north Indian Ocean)

Argentina [124,915]; Australia; Bangladesh [604]; Canada [442]; Cape Verde 1slands; Chile [915]; Falkland Islands [915]: including South Georgia; French Southern and Antarctic Territories; French Polynesia; Galapagos Islands; Greenland; lceland; India [38]; Indonesia [217]; Iraq [687]; Japan; D.P.R. Korea [1184]; Korea Republic [1184]; Malaysia: Sabah [723]; Mexico; Myanmar; Namibia; New Zealand; Nicaragua [442]; Norway; Pakistan [930]; Panama [442]; Peru; Russia [27]; South Africa [442]; Spain; Sri Lanka: Svalbard and Jan Mayen Islands; Taiwan; United Kingdom; USA [442]; Uruguay [915]
Balaenoptera physalus (Linnaeus 1758)
1
V
$27,36,620,1147$

Fin Whale, Finback, Common Rorqual

(Occurs in all major oceans, extending to pack ice zones but rare in warmest waters at latitudes less than about $20^{\circ}$ )

Angola; Argentina [109]; Australia; Bangladesh [602]; Brazil; Canada [442]; Ecuador; Faeroe 1slands; Falkland Islands: South Georgia; French Southern and Antarctic Territories; French Polynesia; Greenland [442]; Hong Kong; Iceland; India [433]; Indonesia [217]; Italy; Japan; D.P.R. Korea [1184]; Korea Republic [1184]; Madagascar; Mexico [442]; Myanmar; Namibia; New Zealand; Norway; Pakistan [930]; Poland; Portugal; Russia [27,1147]; South Africa [1020]; Spain; Sri Lanka [437]; Suriname [532]; Svalbard and Jan Mayen Islands; Taiwan; Tunisia; United Arab Emirates; United Kingdom; USA [442]

\section{$\begin{array}{llll}\text { Megaptera novaeangliae (Borowski 1781) } & \text { l } & \text { V } & 27,36,101,379,620,723,1147\end{array}$}

\section{Humphack Whale}

(Found in all major oceans from Arctic to Antarctic, but absent from pack ice zones)

American Samoa; Angola; Argentina [124,915]; Australia; Bangladesh [602]; Bermuda [1043]; Brazil; Canada [442]; Cape Verde Islands; Chile [915]; Congo; Cook Islands; Dominican Republic; Ecuador; Falkland lslands; Fiji; France; Greenland [442]; Iceland; India; Indonesia; Iraq [687]; Japan; D.P.R. Korea [1184]; Korea Republic [1184]; Madagascar; Malaysia: Sarawak [723]; Marshall Islands; Mexico; Mozambique [1017]; Netherlands Antilles; New Caledonia; New Zealand; Northern Marianas; Norway; Pakistan [93C]; Panama [442]; Peru; Poland; Russia [36]; Saint Lucia; Saint Vincent; Samoa; Solomon 1slands; South Africa [1020]; Sri Lanka [437]; Svalbard and Yan Mayen Islands; Tonga; United Kingdom; USA [618]; Vanuatu; Venezuela; Zaire

\section{Family BALAENIDAE}

Balaena glacialis P.L.S. Müller 1776 $1 \quad \mathrm{E}$ $27,36,127,620,916,1147$

(= Eubalaena glacialis)

(except subspecies australis)

\section{Black Right Whale, Northern Right Whale}

(Temperate and sub-polar coastal waters: North Atlantic from Florida to Greenland and from Spain to Svalbard; North Pacific from China Sea to Sea of Okhotsk and from California to Bering Sea, including Hawaii; circumpolar in Southern Hemisphere including South Atlantic, South Pacific, Southern Ocean)

Argentina [109,675,915]; Australia; Azores; Bermuda; Brazil; Canada (442); Chile [915]; China; Falkland Islands [915]; France; French Southem and Antarctic Territories: Amsterdam Island, Crozet Islands; Iceland; Ireland; Japan; Korea Republic; Madeira; Mexico [442]; New Zealand: Campbell Island, Cbatham Islands; Russia [106]; Saint Helena: Tristan da Cunha Islands; South Africa [416, 1020]; Spain; Svalbard and Yan Mayen Islands; Taiwan; United Kingdom; USA [442]; Uruguay [915] 


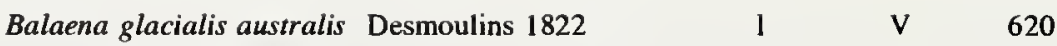

(= Eubalaena australis)

Southern Right Whale

(Circumpolar in Southern Hemisphere including South Atlantic, South Pacific, Southem Ocean)

Argentina [109, 124,675]; Australia; Brazil; Chile; Falkland Islands; French Southern and Antarctic Territories: Amsterdam Island; Crozet Islands, New Zealand: Campbell Island, Chatham Islands; South Africa [4I6,1020]; Tristan da Cunha Islands

Balaena mysticetus Linnaeus 1758

I V

$27,36,127,620,754,917,1147$

Bowhead Whale, Greenland Right Whale

(Arctic Ocean, Beaufort, Barents and Chukchi Seas; North Atlantic - arctic waters; North Pacific - arctic waters, Japan, Okhotsk and Bering Seas)

Canada: including Hudson Bay, Davis Strait, Baffin Island [442]; Greenland; Iceland; Japan; Norway; Russia [106]; Svalbard and Jan Mayen Islands; USA [442]

$\begin{array}{llll}\text { Caperea marginata (Gray 1846) } & \text { I } & \text { K } & 36,185,620,1147\end{array}$

Pygmy Right Whale

(Circumpolar in Southern Hemisphere, north of Antarctic Convergence; northern limit is probably the $20^{\circ} \mathrm{C}$ isotherm)

Argentina [915]; Australia; Falkland Islands [915]; French Southern and Antarctic Territories; New Zealand; South Africa [1020]

\section{Order CARNIVORA}

\section{Family CANIDAE}

Canis aureus Linnaeus 1758

III IN $\quad 466$

\section{Golden Jackal}

Afghanistan; Algeria; Armenia; Azerbaijan; Bangladesh; Bhutan; Bulgaria; Djibouti; Egypt; Ethiopja; Georgia; Greece; Hungary; India; Iran; Iraq; Israel; Kenya; Kyrgyzstan; Lao P.D.R. [271]; Lebanon; Libya; Mauritania; Morocco; Myanmar; Nepal [573]; Nigeria; Pakistan; Romania; Saudi Arabia; Senegal; Somalia; Sri Lanka; Sudan; Syria; Tajikistan; Tanzania; Thailand; Turkey; Turkmenia; Uganda; Western Sahara; Yemen; Yugoslavia

Canis lupus Linnaeus 1758

Grey Wolf

Appendix I populations $\begin{array}{lll}\text { I } & \mathrm{V} & 1098\end{array}$

Bhutan; India [38,836,997]; Nepal [756]; Pakistan [797,930]
Appendix II populations
II V
$392,460,464,720,731,1098$

Afghanistan [440,793]; Albania; Armenia [392]; Austria (ex); Azerbaijan [392]; Bangladesh (ex?) [393,602,604]; Belarus [392]; Belgium (ex); Bulgaria; Canada [191,442]; China [985,994]; Czechoslovakia [1 139]; Denmark (ex); Estonia [392]; Egypt [464]; Finland; France (ex); Germany (ex); Greece; Greenland [442]; Hungary (ex); Iran [646]; Iraq [464,687]; Ireland (ex) [338]; Israel [730]; Italy; Japan (ex); Jordan [464]; Kazakhstan [392]; D.P.R. Korea [1184]; ?Korea Republic [1184]; Kuwait [464]; Kyrgyzstan [392]; Latvia [392]; Lebanon [464,630]; Libya [346]; Lithuania [392]; Luxembourg (ex); Mexico [442,906]; Moldova [392]; Mongolia [695]; Myanmar (ex?) [955]; Netherlands (ex); Norway [749,1140]; Oman [464]; Poland; Portugal [16,357]; Romania; Russia [392]; Saudi Arabia [786]; Slovenia [842]; Spain [274]; Sweden [137,750]; Switzerland (ex); Syria [464,630]; Tajikistan [392]; Turkey [629,1106]; Turkmenia [392]; Ukraine [392]; United Kingdom (ex); USA [191,442]; Uzbekistan [392]; Viet Nam [684]; Yemen; Yugoslavia [147] 
( $=C$. niger)

\section{Red Wolf}

USA

Canis simensis Rūppell 1835

Simien Fox, Simian Jackal, Abyssinian Wolf

Ethiopia

Chrysocyon brachyurus (IIliger 1815)

Maned Wolf

Argentina [124,833,915], Bolivia [1198], Brazil, Paraguay [915], Peru [510], Uruguay (ex) [958]

Cuon alpinus (Pallas 1811)

Asiatic Wild Dog, Dhole, Red Dog

Bangladesh [393,602,603,604,970]; Bhutan; Cambodia; China [836,994]; India [38,99,100]; Indonesia: Java, Kalimantan (ex) [723], Sumatra; Kazakhstan; ?D.P.R. Korea; ?Korea Republic; Kyrgyzstan [106]; Lao P.D.R. [271,837,976,977]; Malaysia: Peninsular Malaysia, Sabah (ex), Sarawak (ex); Mongolia [695]; Myanmar [955]; Nepal [756]; ?Sri Lanka (ex?); Tajikistan [106]; Thailand [151]; Turkey; Uzbekistan [106]; Viet Nam $[837,853,1025]$

Dusicyon australis (Kerr 1792)

\section{Falkland Island Wolf}

Falkland Islands (ex) [124]

Dusicyon culpaeus (Molina 1782)

II

392

(includes $D$. culpaeolus (Molina 1782))

Colpeo Fox, Andean Wolf

Argentina [184,640,699,701,833,915]; Bolivia [184,640]; Chile [184,394,640,696,870,915,1069]; ?Colombia [514]; Ecuador [184,640]; Peru [85, 184,420,640]; Unuguay [958]

Dusicyon griseus (Gray 1837)

(includes D. fulvipes Gray 1837)

Argentine Grey Fox, Pampa Fox, Grey Zorro

Argentina [124,699,701,833,915]; Chile [308,394,554,696,746,870,885,915,1069]; Falkland Islands; Peru

Dusicyon gymnocercus (G. Fischer 1814)

II

392

Pampa Fox, Azara's Zorro

Argentina [699,915]; Bolivia [915]; Brazil; Paraguay [915]; Uruguay [915]

Dusicyon microtis (Sclater 1883)

$\mathrm{K}$

$123,392,1098$

(= Atelocynus microtis)

Small-eared Dog, Smalieared Zorro

?Bolivia; Brazil [196]; Colombia [316]; Ecuador; Peru

Argentina [699,834,915]; Bolivia; Brazil; Colombia [316]; Frrench Guiana; Guyana [316]; Paraguay [915];
Dusicyon sechurae Thomas 1900

Sechuran Zorro, Sechura Fox

Ecuador [392]; Peru [392]

Dusicyon thous (Linnaeus 1766)

Forest Fox, Common Zorro

Suriname [316]; Uruguay [915]; Venezuela [63]

Dusicyon vetulus Lund 1842

K

K $\quad 392$

\section{Hoary Zorro, Hoary Fox}

Brazil 
Wild Dog, African Hunting Dog

Algeria (ex?); Angola [518]; Benin (ex?) [978]; Botswana [1020]; Burkina Faso [944]; Burundi (ex?) [1128]; Cameroon [557]; Central African Republic [689]; Chad [689]; Congo (ex?) [691]; Côte d'Ivoire; Djibouti (ex); Ethiopia [1193]; Gabon (ex?) [691]; Ghana (ex?) [83]; Guinea; Kenya [613]; Malawi [78,452, 1066]; Mali (ex?); ?Mauritania; Mozambique [1017,1020]; Namibia [508,1020]; Niger [872]; Nigeria [19,940]; Rwanda (ex?); Senegal [307]; Sierra Leone [1086]; Somalia [337,367]; South Africa [1013, 1020]; Sudan [503,996]; Swaziland [1020]; Tanzania [613, 1067]; Togo (ex?); Uganda (ex?) [120,613]; Zaire (ex?) [991]; Zambia [75]; Zimbabwe [1020]

$\begin{array}{lllll}\text { Speothos venaticus (Lund 1842) } & \text { I } & \text { V } & 392,1098\end{array}$

Bush Dog

Argentina [124,915]; Bolivia [1198]; Brazil [251]; Colombia [316]; Ecuador; French Guiana; Guyana; Panama [442]; Paraguay [915]; Peru; Suriname [316,532]; Venezuela $[316,640,766]$

Urocyon littoralis (Baird 1858)
Island Grey Fox
USA: California

$\begin{array}{llll}\text { Vulpes bengalensis (Shaw 1800) III IN Id } 392 & \end{array}$

Bengal Fox

Bangladesh; India [392]; Nepal [392,573]; Pakistan [392]

Vulpes cana Blanford 1877

II $\quad$ K $\quad 392$

Blanford's Fox, Hoary Fox, Afghan Fox

Afghanistan [476]; Iran [646]; Israel; Oman; Pakistan [797,930]; Turkmenia [392]

$\begin{array}{llll}\text { Vulpes corsac (Linnaeus 1758) } & - & \text { K } & 392\end{array}$

Corsac Fox

Afghanistan; China; Kazakhstan; Kyrgyzstan; Mongolia [695]; Russia; Turkmenia

Vulpes pallida (Cretzschmar 1826) $\quad-\quad$ K 392

Pale Fox

Burkina Faso; Cameroon; Chad; Guinea; Libya; Mali; Niger; Nigeria; Senegal; Sudan

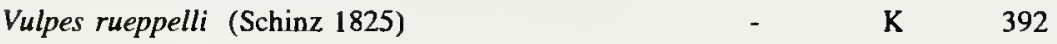

Rueppell's Fox, Sand Fox

Afghanistan; Algeria; Chad; Egypt; Ethiopia; Iraq [687]; Iran; Libya; Morocco; Niger; Saudi Arabia; Somalia; Sudan; Yemen

Vulpes vulpes griffithi Blyth 1854

III IN $\quad-\quad 324$

Afghanistan; Pakistan

Vulpes vulpes montana Pearson 1836

111 IN $\quad-\quad 324$

?Bhutan; China; India; Nepal; Pakistan

Vulpes vulpes pusilla Blyth 1854

III IN $\quad-\quad 324$

(includes V. v. leucopus Blyth 1854)

India; Iran; Iraq; Pakistan

Vulpes zerda (Zimmermann 1780)

II $\quad$ K $\quad 4,392,724$

(=Fennecus zerda)

Fennec Fox

Algeria [942]; ?Burkina Faso [944]; Chad [801]; Egypt [835]; Israel; Kuwait [464]; Libya [528,942];

Mauritania; Morocco [183,856]; Niger [805]; Oman; Sudan; Tunisia [942]; Western Sahara [1113] 
Family URSIDAE

Ailuropoda melanoleuca (David 1869)
Giant Panda
China
Helarctos malayanus (Raffles 1821)

\section{Sun Bear}

Bangladesh (ex) $[602,603,604,970]$; Brunei [119, 170]; Cambodia; China [671,994]; India [38, 384]; Indonesia: Kalimantan [1009], Sumatra [934]; Lao P.D.R. [271,417,837,976]; Malaysia: Peninsular Malaysia, Sabah [266], Sarawak; Myanmar [955]; Thailand [15I]; Viet Nam [837,853,1025]

Melursus ursinus (Shaw 1791)

I V

\section{Sloth Bear}

Bangladesh [602,603,604]; ?Bhutan; India [38,99, 100,884]; Nepal [756]; Sri Lanka

$\begin{array}{lllll}\text { Selenarctos thibetanus (G. Cuvier 1823) } & \text { I } & \text { V } & 514\end{array}$

(= Ursus thibetanus)

(except subspecies gedrosianus)

\section{Asiatic Black Bear}

Afghanistan [440,476]; Bangladesh [602,603,604,970]; Bhutan; Cambodia; China [671,994,1 169]; India [381,884]; Japan; D.P.R. Korea [1184]; Korea Republic [1184]; Lao P.D.R. [271,417,837,976]; ?Malaysia: Peninsular Malaysia; Mongolia; Myanmar [955]; Nepal [756]; Pakistan [797,930]; Russia [106]; Taiwan; Thailand [15I]; Viet Nam [837,853,1025]

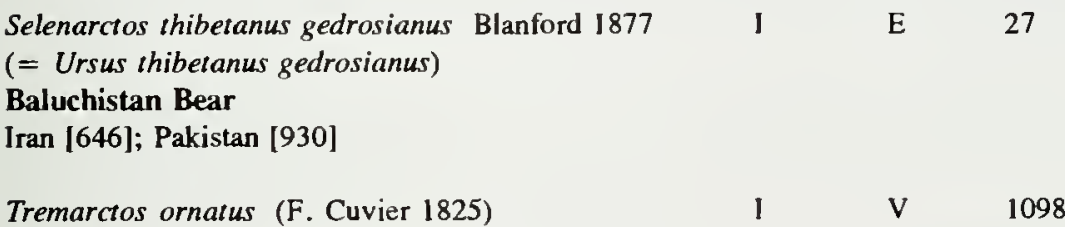

\section{Spectacled Bear}

Argentina; Bolivia [184]; Brazil; Colombia [184,316]; Ecuador; Panama; Peru [184,316,420]; Venezuela [184,316]

Ursus americanus Pallas 1780

American Black Bear

Canada; Mexico [906]; USA

Ursus arctos Linnaeus 1758

(except subspecies isabellinus, nelsoni)

Brown Bear, Grizzly Bear

\section{Appendix I populations}

Bhutan; China; Mongolia

Appendix II populations
$11 \quad$ - 514

Albania; Armenia [106]; Austria (ex); Azerbaijan [106]; Belarus; Belgium (ex); Bulgaria; Canada [442]; Czechoslovakia [949]; Denmark (ex); Finland; France [340]; Georgia [106]; Germany (ex) [139]; Greece; Hungary; Iran; Iraq; Israel (ex); Italy; Japan; Jordan (ex); D.P.R. Korea; Latvia; Lebanon (ex); Liechtenstein (ex); Luxembourg (ex); Netherlands (ex); Norway [623]; Poland; Portligal (ex); Romania; Russia; Spain [274,378]; Sweden; Switzerland (ex); Syria (ex); Turkey; Turkmenia; United Kingdom (ex); USA [442, 852]; Yugoslavia 
Himalayan Brown Bear

Afghanistan [793]; China [671]; India [38,381,836]; Kazakhstan [106]; Kyrgyzstan [106]; Nepal; Pakistan [797,930]; Tajikistan [106]; Uzbekistan [106]

Ursus arctos nelsoni Merriam 1914

Mexican Grizzly Bear

\begin{tabular}{|c|c|c|}
\hline $\begin{array}{l}\text { Appendix I populations } \\
\text { Mexico (ex) [442] }\end{array}$ & I & Ex \\
\hline $\begin{array}{l}\text { Appendix II populations } \\
\text { USA (ex) }\end{array}$ & Il & Ex \\
\hline Ursus maritimus Phipps 1774 & II & $v$ \\
\hline
\end{tabular}

(= Thalarctos maritimus)

Polar Bear

Canada [442]; Greenland [442]; Iceland; Japan; Norway; Russia [106]; Svalbard and Jan Mayen Islands; USA [442]

Family PROCYONIDAE
Ailurus fulgens F. Cuvier 1825
$11 \quad \mathrm{~K}$
884,929

Lesser Panda, Red Panda

Bhutan; China [994]; India [38]; Lao P.D.R. [271,417,976]; Myanmar [955]; Nepal [756]

$\begin{array}{lll}\text { Bassaricyon gabbii J.A. Allen } 1876 & \text { III CR } & -\end{array}$

Bushy-tailed Olingo

Colombia [184,316]; Costa Rica [442]; Ecuador [184]; Honduras [703]; Nicaragua [442]; Panama [442]; Peru; Venezuela $[316,448]$

Bassariscus sumichrasti (Saussure 1860)

III CR - $\quad 442$

\section{Central American Cacomistle}

Belize [617,716]; Costa Rica [442]; El Salvador [178,442]; Guatemala [404,442]; Honduras [442,703]; Mexico [442,906]; Nicaragua [442]; Panama [316,442]

Nasua nasua (Linnaeus 1766)

III HN -

184,442

(includes $N$. narica Linnaeus 1766)

(except subspecies solitaria)

Coati

Belize; Bolivia [1198]; Brazil; [Chile: Robinson Crusoe 1sland [915]]; Colombia [316]; Costa Rica; ?Ecuador; El Salvador [178]; French Guiana [316]; Guatemala [404]; Guyana [316]; Honduras [703]; Mexico [906]; ?Nicaragua; Panama [316]; Paraguay [915]; ?Peru; Suriname [316]; USA; Venezuela [316]

Mexico: Cozumel 1sland

\section{Kinkajou}

?Belize; Brazil; Colombia [316]; Costa Rica; ?Ecuador; El Salvador [178]; French Guiana [316]; Guatemala [404]; Guyana [316]; Honduras [703]; Mexico [906]; ?Nicaragua; Panama [316]; ?Peru; Suriname [316]; Venezuela [316] 
Procyon gloveralleni Nelson \& Goldman 1930

(=P. lotor gloveralleni)

Barbados Raccoon

Barbados (ex?)

Procyon pygmaeus Merriam 1901

(= P. lotor pygmaeus)

Cozumel Island Raccoon

Mexico: Cozumel Island

\section{Family MUSTELIDAE}

Aonyx capensis (Schinz 1821)

African Clawless Otter, Cape Clawless Otter

Angola [518]; Benin; Botswana [1019, 1020]; Burkina Faso [944]; Burundi; Cameroon [942]; ?Central African Republic; Chad [689]; ?Congo; Côte d'lvoire; Equatorial Guinea: Fernando Po [942,1093]; Ethiopia [942]; Gabon; Gambia [942]; Ghana [942]; Guinea; Guinea-Bissau [942]; Kenya; Liberia [14,628]; Malawi [78,452]; ?Mali; Mozambique [1017,1020]; Namibia [1020]; Niger [872]; Nigeria [942]; Rwanda; Senegal [307,942]; Sierra Leone [837,1086]; South Africa [1020]; Sudan [942]; Swaziland; Tanzania [462,613]; ?Togo; Uganda [120]; Zaire; Zambia [75]; Zimbabwe [1020]

Aonyx cinerea (Illiger 1815)

Oriental Small-clawed Otter

Bangladesh [602,604]; Bhutan; Brunei; Cambodia; China [345, 1073,1169]; India; Indonesia: Java, Kalimantan, Sumatra [934]; Lao P.D.R. [271,417,837]; Malaysia: Peninsular Malaysia [721,1151], Sabah [266,468], Sarawak; Myanmar [955]; Nepal [756]; Philippines: Palawan; Singapore [469]; Sri Lanka; Thailand [96,151]; Viet Nam $[837,1025]$

Aonyx congica Lönnberg 1910

(= A. microdon (Pohle 1920))

Cameroon Clawless Otter (Appendix I populations) $\quad 1 \quad$ - $\quad 27,724$

Cameroon [942]; Nigeria

Zaire Clawless Otter (Appendix II populations)

$11 \quad$ - 724

Angola; Burundi; Congo [691]; ?Equatorial Guinea; Gabon [691]; Rwanda; Uganda [613]; Zaire [233,896]

Conepatus humboldtii Gray 1837

Il

184

(includes $C$. castaneus)

Patagonian Hog-nosed Skunk

Argentina [196,833,915]; Chile [366,394,915, 1069]; ?Paraguay [514]

Conepatus mesoleucus telmalestes V. Bailey 1905

Big Thicket Hog-nosed Skunk

USA: Texas [992]

Eira barbara (Linnaeus 1758)

\section{Tayra}

Argentina [699,915]; Belize; Bolivia [1198]; Brazil; Colombia [316]; Costa Rica; Ecuador; El Salvador [178]; French Guiana [316]; Guatemala [404]; Guyana [316]; Honduras [703]; Mexico [205,906]; Nicaragua; Panama [316]; Paraguay [167,915]; ?Peru; Suriname [316]; Trinidad and Tobago [13]; Uruguay [177,958]; Venezuela $[316,319]$

Enhydra lutris (Linnaeus 1758)

II

Id

442

(except subspecies nereis)

Sea Otter

Japan [972]; Russia: Kurile Islands [106]; USA: Alaskan south-east coast, including Aleutian and Commander Islands 
Canada: Vancouver and Straits of Juan de Fuca; Mexico: west coast (ex) [205,906]; USA: west coast [59]

Galictis vittasa (Schreber 1776)

III CR -

442

(includes G. allamandi)

Greater Grison

Belize [716]; Bolivia [1198]; Brazil; Colombia [316]; Costa Rica [442]; Ecuador; El Salvador [442]; ?French Guiana; Guatemala [442]; Guyana [316]; Honduras [442,703]; Mexico [205,442,906]; Nicaragua [442]; Panama [316,442]; Peru; Surinarne [316]; Venezuela [316]

Gulo gulo (Linnaeus 1758)

V 992

Wolverine

Canada [992]; Estonia [992]; Finland [992]; Mongolia [695,992]; Norway [131,992]; Russia [992]; Sweden [992]; USA [992]

Lutra canadensis (Schreber 1776)

II

442,561

North American Otter

Canada; Mexico (ex) [205]; USA

Lutra felina (Molina 1782)

I

V

184,1098

Marine Otter, Chingungo

Argentina [124,833,915]; Chile $[201,394,746,915]$; Penu

Lutra longicaudis (Olfers 1818)

1

$184,331,462$

(except subspecies longicaudis)

(includes $L$. annectens, $L$. enudris, $L$. incarum)

South American River Otter, Neotropical River Otter

Belize [617,716]; Bolivia [1198]; Brazil; Colombia [291,316]; Costa Rica [442]; Ecuador [727]; El Salvador [178,442]; French Guiana; Guatemala [442]; Guyana [316]; Honduras [442,703]; Mexico [205,442,906]; Nicaragua [442]; Panama [442]; Penu [163]; Suriname [316,532]; Trinidad and Tobago [13]; Venezuela $[316,1201]$

Lutra longicaudis longicaudis (Olfers 1818)

$1 \quad \mathrm{~V}$

$184,462,1098,1207$

(= L. platensis (Waterhouse 1838))

\section{La Plata Otter}

Argentina [699,701,833,915]; Bolivia; Brazil [251]; ?Paraguay [915]; Unguay [915,958]

Lutra lutra (Linnaeus 1758)

1

$96,323,462$

(except subspecies lutra)

Eurasian Otter

Afghanistan [440,476,793]; Bangladesh [602,604]; Bhutan; Cambodia; China [994,1073,1169]; Hong Kong [709]; India [836]; Indonesia: Sumatra [934]; 1ran [646]; 1raq [687]; Japan [972]; Kazakhstan [106]; Kyrgyzstan; [106]; Lao P.D.R. [271,417]; Malaysia [721]; Myanmar [955]; Nepal [756]; Pakistan [797,930]; Slovenia [842]; Sri Lanka [1151]; Taiwan; Tajikistan [106]; Thailand [151,216]; Turkmenia [106]; Uzbekistan [106]; Viet Nam [837,853,1025]; Yugoslavia: Slovenia [1132]

Lutra lutra lutra (Linnaeus 1758)

l V

$27,462,777$

\section{European Otter}

Albania [681]; Algeria; Armenia [323]; Austria; Azerbaijan [323]; Belarus [323]; Belgium [249]; Bulgaria; China; Czechoslovakia [523]; Denmark [1045]; Estonia [323]; Finland; France [409]; Georgia [323]; Germany [924, 1054]; Greece [675,680]; Hungary; Ireland [213]; Israel [681,682]; Italy [198,677,1151]; Jordan [464]; Kazakhstan [323]; D.P.R. Korea [1184]; Korea Republic [1184]; Latvia [323]; Lebanon [630,653]; Liechtenstein (ex); Lithuania [323]; Luxembourg (ex); Moldova [323]; Mongolia [695]; Morocco [679]; Netherlands [710]; Norway [484]; Poland; Portugal [16,115,676]; Romania; Russia [323]; Spain [274,325]; Sweden [328]; Switzerland (ex); Syria [630]: Tunisia [678]; Turkey [629,1106]; Ukraine [323]; United Kingdom [674]; Western Sahara [1113]; Yugoslavia [658] 
(= Hydrictis maculicollis)

\section{Spot-necked Otter, Speckle-throated Otter}

Angola [518]; Botswana [1019,1020]; Burkina Faso [944]; Burundi; Cameroon [942]; Chad [689]; Congo [691]; Côte d'Ivoire; ?Egypt; Equatorial Guinea [182,942]; Ethiopia [942]; Gabon [691]; ?Guinea; ?Iran; Kenya; Liberia [14,628,942]; Malawi [78,452]; Mozambique [1017,1020]; Namibia [1020]; Niger; Nigeria; Rwanda; Sierra Leone [942,1086]; South Africa [1020,1052]; Sudan [942]; ?Swaziland; Tanzania [886]; Uganda [120]; Zaire [233,896,991]; Zambia [75]; Zimbabwe

\section{Lutra perspicillata I. Geoffroy 1826 \\ II $\quad \mathrm{K}$ \\ $323,462,723$}

Smooth-coated Otter

Bangladesh [602,604,970]; Bhutan [1115]; Cambodia; China [1073]; India; Indonesia: Java [1114], Kalimantan, Sumatra; Iraq [464,687]; Lao P.D.R. [837]; Malaysia: Peninsular Malaysia [721,1151], Sabah [266], Sarawak; Myanmar [955]; Nepal [573,756]; Pakistan [797,930]; Thailand [96,151]; Viet Nam [853,1025]

Lutra provocax Thomas 1908

Southern River Otter, Huillin

Argentina [124,218,833,915]; Chile [394,746,915]

Lutra sumatrana (Gray 1865)

Hairy-nosed Otter

Brunei; Cambodia; Indonesia: Bangka [217], Kalimantan, Sumatra [934]; ?Lao P.D.R.; Malaysia: Peninsular Malaysia [721], Sabah [266], Sarawak; Singapore [469]; Thailand [96,151]; Viet Nam [837,853,1025]

Martes flavigula (Boddaert 1785)

III IN $\quad-\quad 992$

(except subspecies chrysospila, gwatkinsi)

Yellow-throated Marten

Bhutan [953]; China; India; Indonesia: Java, Sumatra; D.P.R. Korea; Korea Republic; Lao P.D.R. [271]; Malaysia: Peninsular Malaysia, Sabah, Sarawak; Myanmar; Nepal [573]; Pakistan; Russia; Thailand; Viet Nam

Martes flavigula chrysospila Swinhoe 1866

III IN Id

992

Taiwan Yellow-throated Marten

Taiwan

Martes flavigula gwatkinsi Horsfield 1851

III IN Id

992

(= M. gwatkinsi)

Nilgiri Marten

India

Martes foina intermedia Severtzov 1873

III IN

239

Central Asian Stone Marten

Afghanistan; China; India; Iran; Kyrgyzstan; Pakistan; Turkmenia

Martes melampus tsuensis Thomas 1897

Id

992

Tsushima Island Marten

Japan [992]

Mellivora capensis (Schreber 1776)

Honey Badger, Ratel

III BW

GH

?Afghanistan [440,476]; Algeria; Angola [518]; ?Benin; Botswana [1020]; Burkina Faso; Cameroon [942];

Central African Republic [689]; Chad [801]; Congo [691]; Côte d'Ivoire [515]; Egypt (ex) [239]; Equatorial

Guinea [942]; Ethiopia; Gabon [691]; Ghana [942]; Guinea; India [38]; Iran [646]; Iraq [464,687]; Israel [464,734]; ?Jordan; Kazakhstan [106]; Kenya [613]; Kuwait [464]; ?Lesotho; Liberia [628]; Malawi [78]; Mali; 
Mauritania; Morocco [183]; Mozambique [1017,1020]; Namibia [1020]; Nepal [756]; Niger [805,942]; Nigeria; Oman [464]; Pakistan [930]; Rwanda; Saudi Arabia [464]; Senegal [942]; Sierra Leone [942]; Somalia [337,367]; South Africa [725,1020]; Sudan [503]; ?Swaziland; Tanzania [613]; ?Togo; Turkmenia [106]; Uganda [120]; Uzbekistan; Western Sahara [1113]; Yemen [464]; Zaire [233,896]; Zambia [75]; Zimbabwe [1020]

Melogale everetti (Thomas 1895)

Kinabalu Ferret-badger, Everett's Ferret-badger

Malaysia: Sabah

Melogale personata orientalis Horsfield 1821

$(=M$. orientalis)

Javan Ferret-badger

Indonesia: Java

Mustela altaica Pallas 1811

Mountain Weasel

China; India; Kazakhstan; D.P.R. Korea; Korea Republic; Mongolia; Nepal; Russia

Mustela erminea Linnaeus 1758

III IN

K

992,1118

\section{Stoat, Ermine}

Afghanistan; Algeria; Belarus; Belgium; Canada; China; Czechoslovakia; Denmark; Estonia; Finland; France; Germany; Hungary; India; lreland; Japan; Kazakhstan; Kyrgyzstan; Latvia; Lithuania; Moldova; Mongolia; Netherlands; Norway; Pakistan; Poland; Russia; Spain; Sweden; Switzerland; Tajikistan; Turkmenia; Ukraine; United Kingdom; USA

Mustela kathiah Hodgson 1835

III JN

514

Yellow-bellied Weasel

Bhutan; China; India; Lao P.D.R. [271]; Myanmar; Nepal; Pakistan; Viet Nam

Mustela lutreola (Linnaeus 1761)

$$
\text { V } \quad 267,1199
$$

\section{European Mink}

Austria (ex?) [992]; Belarus [1005]; Bulgaria [160,992]; Czechoslovakia [992]; Finland (ex?) [992]; France [992]; Germany [992]; Greece; Hungary (ex?) [992]; Latvia [1092]; Lithuania [741]; Netherlands (ex); Poland [992]; Romania [992]; Russia [992]; Spain [992]; Sweden; Yugoslavia [992]

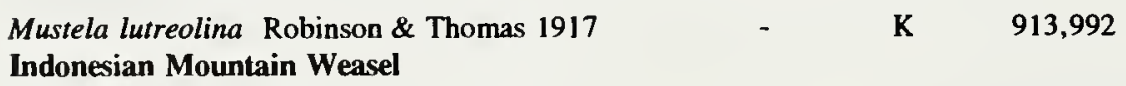

Indonesia: Java, Sumatra

Mustela nigripes (Audubon \& Bachman 1851)

I

$\mathrm{E}$

$58,70,231,442,502,743,922$, 1098,1154

Black-footed Ferret

Canada (ex); USA

Mustela sibirica Pallas 1773

III IN

514,239

Siberian Weasel

Bhutan; China; India; Indonesia: Java; Japan; D.P.R. Korea; Korea Republic; [Kyrgyzstan]; Myanmar; Nepal; Russia; Taiwan

Pteronura brasiliensis (Gmelin 1788)

l V

$184,462,1098$

Giant Otter, Giant Brazilian Otter

Argentina [124,833,915]; Bolivia [1198]; Brazil [251]; Colombia [270,316]; Ecuador; French Guiana [316]; Guyana [316]; Paraguay [915]; Peru; Suriname [316,532]; Uruguay (ex?) $[915,958]$; Venezuela [316] 
Vormela peregusna peregusna (Güldenstädt 1770) - $\quad$ V 160

European Marbled Polecat

Bulgaria [992]; Greece [992]; Romania [992]; Turkey [992]; Ukraine [992, 1068]; Yugoslavia [992]

\section{Family VIVERRIDAE}

Arctictis binturong (Raffles 1821)

IIJ JN -

514

\section{Binturong}

Bangladesh; China; India; Indonesia: Java, Sumatra; Lao P.D.R. [271]; Malaysia; Myanmar; Philippines: Palawan; Thailand; Viet Nam

Arctogalidia trivirgata trilineata Wagner $1814 \quad$ - $\quad$ ld $\quad 913,992,1118$

Javan Small-toothed Palm Civet

Indonesia: Java

Chrotogale owstoni Thomas 1912

K 992

Owston's Palm Civet

China $[945,992]$; Lao P.D.R. $[271,945,992]$; Viet Nam $[807,992]$

Civettictis civetta (Schreber 1777)

$111 \mathrm{BW}-$

724

(= Viverra civetta)

\section{African Civet}

Angola [518]; ?Benin; Botswana [1020]; Burkina Faso [944]; ?Burundi; Cameroon [557]; Central African Republic [689]; Chad [801]; Congo [691]; Côte d'lvoire [515,1178]; Equatorial Guinea [182]; Ethiopia [245,942]; Gabon [691]; Gambia [574]; ?Ghana; ?Guinea; Kenya [613]; Liberia [14,628]; Malawi [78,452,1066]; Mali; Mozambique [1017,1020]; Namibia [1020]; ?Niger; Nigeria [20]; Rwanda; [São Tome and Principe [365]]; Senegal [942]; Sierra Leone [263,264,1086]; Somalia [337,367]; South Africa [725,1020]; Sudan [503,942]; ?Swaziland; Tanzania [613,771]; ?Togo; Uganda [120]; Zaire [233,896]; Zambia [75]; Zimbabwe [1020]

Cryptoprocta ferox Bennett 1833

Fossa

Madagascar

Cynogale bennettii Gray 1837

(includes C. lowei Pocock 1933)

Otter-civet

Brunei; Indonesia: Kalimantan, Sumatra [934]; Malaysia: Peninsular Malaysia, Sabah [266], Sarawak; ?Singapore [469]; Thailand [151]; Viet Nam [151,837,1025]

Eupleres goudorii Doyere 1835

IJ

(includes E. major Lavauden 1929)

Falanouc

Madagascar

Fossa fossa (Schreber 1777)

11

(=F. fossana (P.L.S. Müller 1776))

Malagasy Civet, Striped Civet

Madagascar

Genetta abyssinica (Rüppell 1836)

Abyssinian Genet

Djibouti [992]; Ethiopia [992]; Somalia [992]

Genetta genetta isabelae Delibes 1977

\section{Ibiza Genet}

Spain: Ibiza
$11 \quad \mathrm{~K} \quad 6,9,27,622$

$11 \quad \mathrm{~K} \quad 723$ 
Johnston's Genet

Côte d'Ivoire [515,638]; Liberia [992]; Guinea [992]

Hemigalus derbyanus (Gray 1837)

II

Banded Palm Civet

Brunei [170]; Indonesia: Kalimantan [1009], Mentawai Islands, Sumatra [934]; Malaysia: Peninsular Malaysia, Sabah [266], Sarawak; Myanmar; Thailand [151]

Macrogalidia musschenbroekii (Schlegel 1879) $\begin{array}{lll}- & \mathrm{R} & 27,1156\end{array}$

Sulawesi Palm Civet, Musang, Brown Palm Civet

Indonesia: Sulawesi

Paguma larvata (H. Smith 1827)

$111 \mathrm{IN}$

Masked Palm Civet

Bangladesh; China; India: including Andaman Islands; Indonesia: Sumatra; Lao P.D.R. [271]; Malaysia: Peninsular Malaysia, Sabah; Myanmar; Nepal; Sri Lanka; Taiwan; Thailand; Viet Nam

Paradoxurus hermaphroditus (Pallas 1777)

III IN

244

(except subspecies lignicolor)

Common Palm Civet

Bangladesh; Bhutan; China; India; Indonesia; Lao P.D.R. [271]; Malaysia: Peninsular Malaysia, Sabah, Sarawak; Myanmar; Nepal [573]; Philippines; Sri Lanka; Thailand; Viet Nam
Paradoxurus hermaphroditus lignicolor Miller 1903
III IN E
913,992
$(=P$. lignicolor $)$
Mentawai Palm Civet
Indonesia: Sumatra

Paradoxurus jerdoni Blanford 1885

Ill IN Id 992

Jerdon's Palm Civet

India [904]

Poiana richardsoni liberiensis Pocock 1908

Leighton's Linsang

Liberia [992]; Côte d'lvoire [515,992]; Sierra Leone [992]

Prionodon linsang (Hardwicke 1821)

\section{Banded Linsang}

Brunei; Indonesia: Bangka [217], Belitung, Java [217], Kalimantan, Sumatra [934]; Malaysia: Peninsular Malaysia [469], Sabah [266], Sarawak; Myanmar [955]; Thailand [151]

Prionodon pardicolor Hodgson 1842

1

884

Spotted Linsang

Bhutan; China [994, 1073]; India [38]; Lao P.D.R. [271,417,837]; Myanmar [955]; Nepal [756]; Thailand [151];

Viet Nam $[837,853,1025]$

Viverra megaspila Blyth 1862

III IN $\quad-\quad 992$

(except subspecies civettina)

Large-spotted Civet

Lao P.D.R. [271]; Malaysia: Peninsular Malaysia; Myanmar; Thailand; Viet Nam

Viverra megaspila civettina Blyth 1862 III IN E $27,38,591,631,884$

( = V. civettina)

Malabar Large-spotted Civet

India: Kerala 


\section{Large Indian Civet}

Bangladesh; Cambodia; China; India; Lao P.D.R. [271]; Malaysia: Peninsular Malaysia; Myanmar; Nepal; Thailand; Viet Nam

Viverricula indica (Desmarest 1817)

III IN

\section{Small Indian Civet}

Bangladesh; Bhutan; Cambodia; China; India; Indonesia: Bali, Java, Kangean Islands, Sumbawa; Lao P.D.R. [271]; [Madagascar]; Malaysia: Peninsular Malaysia; Myanmar; Nepal; [Socotra]; Sri Lanka; Taiwan; [Tanzania]; Thailand; Viet Nam

\section{Family HERPESTIDAE}

Bdeogale crassicauda omnivora Heller 1913

Sokoke Bushy-tailed Mongoose

Kenya [992]; Tanzania [992]

Bdeogale jacksoni (Thomas 1894)

K

Jackson's Mongoose

Kenya [992]; Uganda [992]

Galidictis fasciata (Gmelin 1788)

Broad-striped Mongoose

Madagascar

Galidictis grandidieri Wozencraft 1986

Giant-striped Mongoose

Madagascar

Herpestes auropunctalus (Hodgson 1836)

$111 \mathrm{IN}$

ld

Id

K

\section{Small Indian Mongoose}

Afghanistan; [Antigua and Barbuda]; Bangladesh; [Barbados]; China; [Cuba]; [Dominican Republic]; [Fiji]; [French Guiana]; [Grenada]; [Guadeloupe]; [Guyana]; [Haiti]; India; Iran; Iraq; [Jamaica]; Lao P.D.R. [271]; Malaysia: Peninsular Malaysia; [Martinique]; Myanmar; Nepal [573]; [Netherlands Antilles: Saint Martin]; Pakistan; [Puerto Rico]; [Saint Kitts and Nevis]; [Saint Lucia]; [Saint Vincent]; Saudi Arabia; [Suriname]; [Tanzania: Mafia 1sland]; Thailand; [Trinidad and Tobago [13]]; [Hawaiian 1slands]; [Virgin Islands of the United States]; Viet Nam

Herpestes edwardsi (E. Geoffroy 1818)

III IN 466

Indian Grey Mongoose

Afghanistan; Bangladesh; India; Iran; Iraq; [Malaysia: Peninsular Malaysia]; Nepal [573]; Pakistan; Sri Lanka

Herpestes fuscus Waterhouse 1838

III IN

514

Indian Brown Mongoose

India; Sri Lanka

Herpestes smithii Gray 1837

III IN

Ruddy Mongoose

India; Sri Lanka

Herpestes urva (Hodgson 1836)

III IN

Crab-eating Mongoose

China; India; Lao P.D.R. [271]; Myanmar; Nepal; Taiwan; Thailand; Viet Nam

Herpestes vitticollis Bennett 1835

III IN

Stripe-necked Mongoose

India; Sri Lanka 
Madagascar

Salanoia concolor (I. Geoffroy 1837)

Brown-tailed Mongoose, Salano

Madagascar

Family PROTELIDAE

Proteles cristatus (Sparrmann 1783)

III BW

724

Aardwolf

Angola [518]; Botswana [1020]; ?Burundi; ?Central African Republic [689]; Egypt [835]; Ethiopia [245]; Kenya [613]; Lesotho [1020]; Mozambique [1017,1020]; Namibia [1020]; Somalia [337,367]; South Africa [1013,1020]; Sudan; Swaziland [1020]; Tanzania [613]; Uganda [120,613]; Zambia [75]; Zimbabwe [1020]

Family HYAENIDAE

Hyaena brunnea Thunberg 1820 1 $\mathrm{V}$ $27,76,312,724,747$

\section{Brown Hyaena}

Angola [518,531]; Botswana [1020]; Mozambique [1017]; Namibia [1020]; South Africa [1013,1020,1053]; Zimbabwe [1020]

Hyaena hyaena barbara Blainville 1844 E $\quad 27,724,925$

Barbary Hyaena

Algeria; Morocco [183]; Tunisia

Family FELIDAE

Acinonyx jubatus (Schreber 1776)

(except subspecies venaticus)

African Cheetah

Appendix I populations

I

$\mathrm{V}$

$27,435,436,724,1018$

Algeria (ex?) [804]; Angola [518]; Benin [978]; Burkina Faso [944]; Burundi (ex) [1128]; Cameroon [557]; Chad [689,804]; Egypt (ex) [804,835]; Ethiopia [1193]; Kenya [33,444,613]; Libya [528]; Malawi [78, 1066]; Mali [804]; Mauritania [804]; Morocco (ex?); Mozambique [1017,1020,1089]; Niger [418,805,872]; Nigeria [19,610]; Senegal [304,804]; Somalia [337,367]; South Africa [748, 1013,1020]; Sudan [503]; Swaziland [156]; Tanzania [33,613]; Tunisia (ex); Uganda [33, 120,613]; Western Sahara (ex?) [804,1113]; Zaire [991]; Zambia [75]

$\begin{array}{llll}\text { Appendix I populations (export quota) } & \text { I(eq) } & \text { V } & 1014\end{array}$

Botswana [1020]; Namibia [704,1020]; Zimbabwe [704,1020,1176]

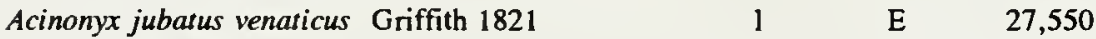

Asiatic Cheetah

Afghanistan (ex?) [440]; India (ex) [38]; Iran [66,590,646]; Iraq (ex?) [464,732]; 1srael (ex) [464]; Jordan (ex?) [464]; Kazakhstan (ex) [106]; Kuwait (ex) [464]; Kyrgyzstan (ex) [106]; Pakistan (ex?) [930]; Saudi Arabia (ex?); Syria (ex) [464,630]; Tajikistan (ex) [106]; Turkmenia (ex) [106]; Uzbekistan (ex) [106]; Yemen (ex) [465] 
Felis aurata Temminck 1827

11

$436,449,724,941,1179$

Golden Cat, African Golden Cat

Angola [518,942]; Burkina Faso; Burundi; Cameroon [736,942]; Central African Republic; Congo [1031]; Côte d'Ivoire [1178]; Equatorial Guinea [182,942]; Gabon [691]; Gambia [443]; Ghana [736]; ?Guinea; Kenya [613]; Liberia [14,628,942]; ?Niger; Rwanda [322,436]; Senegal [736,942]; Sierra Leone [263,736,942]; ?Togo; Uganda [120,613]; Zaire [736,896]

Felis badia Gray 1874

Il R

$27,582,723$

Bay Cat, Bornean Marbled Cat

Brunei; Indonesia: Kalimantan; Malaysia: Sabah [468,1084], Sarawak

Felis bengalensis Kerr 1792

II $\quad-\quad 217,436,550,723$

(except subspecies bengalensis)

Leopard Cat

Afghanistan [440,476]; Bhutan [207]; Brunei; China [669, 1169]; Hong Kong [709]; India [38, 136]; Indonesia: Bali, Java, Kalimantan, Nias, Sumatra [934]; Japan; D.P.R. Korea [1184]; Korea Republic [1184]; ?Macao; Malaysia: Peninsular Malaysia [721], Sabah [266], Sarawak; Nepal [756]; Pakistan [797,930]; Philippines; Russia [106]; Singapore [469]; Taiwan; Thailand [96,151]; Viet Nam [261,837,853,1025]

Felis bengalensis bengalensis Kerr 1792

Bengal Leopard Cat

Appendix I populations

$1 \quad-\quad 544$

Bangladesh [602,603,604,970]; Cambodia [837]; India [136]; Lao P.D.R. [417,837]; Myanmar [955]; Pakistan [797]; Thailand [216]; Viet Nam [837]

\section{Appendix II populations \\ China [57]}

Felis bieti Milne-Edwards 1892

Chinese Desert Cat

China

Felis canadensis (Kerr 1792)

$(=F$. lynx canadensis, Lynx canadensis $)$

American Lynx

Canada [888]; USA

Felis caracal Schreber 1776

(= Lynx caracal)

(except subspecies michaelis)

Asian Caracal (Appendix I populations)

1

II

11

$436,550,994$

Afghanistan [440]; India [38]; Iran [646]; Jraq [464,687,1091]; Israel [464,732,1155]; Jordan [464]; Kuwait [464]; Lebanon [464,630]; Oman [507]; Pakistan [797,930]; Saudi Arabia [464,785]; Syria [464,630]; Turkey [629,1106]; United Arab Emirates [464]; Yemen [464]
African Caracal (Appendix II populations)
I1
$436,449,724,942,1018$

Algeria [942]; Angola [518]; Benin [978]; Botswana [1020]; ?Burkina Faso; Cameroon [557]; Central African Republic [689]; Chad [689,801]; Congo [1031]; ?Côte d'Ivoire; Djibouti; Egypt [835]; Ethiopia; Gabon [691]; Gambia [482,574,942]; Ghana [942]; ?Guinea; Guinea-Bissau [942]; Kenya [613]; ?Lesotho; ?Liberia; Libya [528]; Malawi [78]; ?Mali; Mauritania; Morocco [183,856,942]; Mozambique [1017,1020]; Namibia [1020]; Niger [805,872,942]; Nigeria [942]; Senegal [307]; Somalia [337,367]; South Africa [1020]; Sudan [503,942]; ?Swaziland; Syria; Tanzania [613]; ?Togo; ?Tunisia; Uganda [120,613]; Zaire [991]; Zambia [75]; Zimbabwe [1020] 
Felis caracal michaelis Heptner 1945

」

$\mathbf{R}$

27,106

(= Lynx caracal michaelis)

Turkmenian Caracal

Kazakhstan [106]; Tajikistan [106]; Turkmenia [106]; Uzbekistan [106]

Felis chaus Güldenstädı 1776

II

550

Jungle Cat, Swamp Cat, Reed Cat

Afghanistan [440,695]; Bangladesh [602,604]; Bhutan; Cambodia; China [239]; Egypt [835]; India [136]; Iran [646]; Iraq [464,687]; Israel [464,732]; Jordan [464]; Kazakhstan [239,436]; Lao P.D.R. [271,417]; Mongolia [695]; Myanmar [955]; Nepal [756]; Pakistan [797,930]; Russia [239,436]; Sri Lanka; Syria [465,630]; Tajikistan [239,436]; Thailand [151]; Turkey [629,1106]; Turkmenia [239,436]; Uzbekistan [239,436]; Viet Nam [837,1025]

Felis colocolo Molina 1810

II

184,436

(includes $F$. pajeros)

Pampas Cat, Chilean Pampa Cat

Argentina [699,701,833,915]; Bolivia; Brazil [251]; Chile [394,746,915,1069]; Ecuador; Paraguay [915]; Peru [79,420]; Uruguay $[79,915,1190]$

Felis concolor Linnaeus 1771

11

252

(except subspecies coryi, costaricensis, couguar)

Puma, Cougar, Mountain Lion, Deer Tiger, Red Tiger

Argentina [699,701,833,915]; Belize [617]; Bolivia [1198]; Brazil [184,251,869]; Canada [442]; Chile [394,696,746,870,915,1069]; Colombia; Ecuador [661]; El Salvador [178,442]; French Guiana; Guatemala [442]; Guyana [24,943]; Honduras [703]; Mexico [442,906]; Nicaragua; Paraguay [915,1129,1162]; Peru [85,851]; Suriname [532]; USA [442]; Uruguay [79,915,1190]; Venezuela $[319,448,1201]$

Felis concolor coryi Bangs 1899

Florida Cougar, Forida Puma

USA

Felis concolor costaricensis Merriam 1901

Costa Rican Puma

Costa Rica; Panama

Felis concolor couguar Kerr 1792

Eastern Puma, Eastern Cougar

Canada; USA

Felis geoffroyi d'Orbigny \& Gervais 1844

Geoffroy's Cat

Argentina [124,699,701,833,915]; Bolivia [1198]; Brazil [251]; Chile [394,746,915]; Paraguay [915,1162]; Uruguay $[79,958]$

Felis guigna Molina 1782

Kodkod, Chilean Cat

Argentina [124,833,915]; Chile [394,746,915,1069]

Felis iriomotensis Imaizumi 1967

(= Prionailurus iriomotensis, $F$. bengalensis iriomotensis)

Iriomote Cat

Japan: Iriomote

Felis jacobita Comalia 1865

Andean Cat, Mountain Cat

Argentina [124,833,915]; Bolivia [1198]; Chile [394,746,870,915]; Peru
E $\quad 1098$

II E $27,546,550$
E $\quad 317,1098$ 
Felis lynx Linnaeus 1758

I]

$348,550,597,1105$

$(=$ Lynx lynx $)$

Eurasian Lynx

Afghanistan [793]; Albania; Austria; Bhutan; Bulgaria (ex); China [345,994]; Czechoslovakia; Finland; France [340,491]; Germany; Greece; Hungary; India [38,228,836]; Iran [646,687]; Iraq [464]; Italy: ?Sardinia [239]; Kazakhstan [106]; D.P.R. Korea [1184]; Kyrgyzstan [106]; Mongolia [695]; Nepal [756]; Norway; Pakistan [797,930]; Poland; Romania; Russia [106]; Spain [46]; Sweden; Switzerland [164,491]; Tajikistan [106]; Turkey [629,1106]; Turkmenia [106]; Uzbekistan [106]; Yugoslavia
Felis manul Pallas 1776
I]
436

Pallas's Cat

Afghanistan [440,476]; Armenia [106]; China [345,994]; India [38]; ]ran [646]; Kazakhstan [106]; Kyrgyzstan [106]; Mongolia [695]; Pakistan [797,930]; Russia [106]; Tajikistan [106]; Turkmenia; Uzbekistan [106]

Felis margarita Loche 1858

II

$331,436,724,941$

(except subspecies scheffeli)

Sand Cat

Algeria [942]; Egypt [835]; ]ran [239]; Israel [732]; Jordan [487]; Kazakhstan [436]; Morocco [239]; Niger [942]; Oman [464]; Qatar [465]; Saudi Arabia [464]; Senegal [436]; Turkmenia [436]; Uzbekistan [436]; Yemen [464]

Felis margarita scheffeli Hemmer 1974

Pakistan Sand Cat

I1 E $27,550,797,930$

Pakistan

Felis marmorata Martin 1837

I Id

$27,436,550,723,853$

Marbled Cat

?Bangladesh [602,603,604,970]; ?Bhutan; Brunei; Cambodia; China [1057]; India [38,136]; Indonesia:

Kalimantan [1009]; Sumatra [934]; Lao P.D.R. [271,417]; Malaysia: Peninsular Malaysia [721], Sabah, Sarawak; Myanmar [955]; Nepal [756]; Thailand [96,151]; Viet Nam [837,853,1025]

Felis nigripes Burchell 1824

I $\quad$ - $\quad 436,443,724$

Black-footed Cat, Small-spotted Cat

?Angola [518]; Botswana [1020]; Namibia [1020]; South Africa [1020]

Felis pardalis Linnaeus 1758

l V

$184,436,1098,1122$

Ocelot

Argentina [124,184,832,833,915]; Belize [442,716]; Bolivia [1 198]; Brazil [251]; Colombia [316]; Costa Rica; Ecuador [184]; El Salvador [178,442]; French Guiana [316]; Guatemala [404,442]; Guyana [316]; Honduras [442,703]; Mexico [442,906]; Nicaragua; Panama [316]; Paraguay [184,915]; Peru; Suriname [316]; Trinidad and Tobago [13]; USA [442,796]; Venezuela [316,319]

Felis pardina Temminck 1824

(= Lynx pardinus, $F$. lynx pardina)

Spanish Lynx, Pardel Lynx

Portugal [16]; Spain [274,514]

Felis planiceps Vigors \& Horsfield 1827

Flat-headed Cat

Brunei [723]; Indonesia: Kalimantan, Sumatra [934]; Malaysia: Peninsular Malaysia [721], Sabah [266], Sarawak; [Singapore [721]]; Thailand [151] 
Felis rubiginosa I. Geoffroy 1831

Rusty-spotted Cat

\section{Appendix I populations \\ India}

\section{Appendix II populations \\ Sri Lanka}

Felis rufa Schreber 1776

(= Lynx rufus)

Bobcat, Bay Lynx

Canada; Mexico [906]; USA

Felis serval Schreber 1776

\section{Serval}

Algeria [239]; Angola [518]; Benin [978]; Botswana [1020]; ?Burkina Faso; Burundi [1179]; Cameroon [942]; Central African Republic [689]; Chad [689]; Congo [1031]; ?Côte d'Jvoire; Djibouti; Ethiopia [245]; Gabon [691]; Gambia; Ghana [942]; Guinea; Guinea-Bissau [942]; Kenya [613]; ?Lesotho; Liberia [14,628,942]; Malawi [78,452,1066]; Mali [942]; Mauritania; Morocco [239]; Mozambique [942, 1017]; Namibia [1020]; Niger [872]; Nigeria [610,942]; Rwanda [322]; Senegal [304,942]; Sierra Leone [942]; Somalia [337,367]; South Africa [1013, 1020, 1052]; Sudan [503]; Swaziland; Tanzania [613]; Togo [942]; Uganda [120,613]; Zaire; Zambia [75]; Zimbabwe [1020]

Felis silvestris Schreber 1777

Il

$239,435,436,442,724,941$

(includes $F$. lybica Forster $1780, F$. ornata Gray 1830)

Wild Cat

Afghanistan [440]; Albania; Algeria [239]; Angola [518]; Armenia; Austria; Azerbaijan; Belgium; Belarus [ 106]; Benin [978]; Botswana [1020]; Bulgaria; Burkina Faso [944]; Burundi; Cameroon; Central African Republic [689]; Chad [801,942]; China; Czechoslovakia; Djibouti; Egypt [835]; Ethiopia [245]; France [340]; Gambia; Georgia; Germany; Ghana [152]; Greece; Guinea; Guinea-Bissau; Hungary; India [38]; Iran [646]; Iraq [464,687]; Israel [464,732]; Italy; Jordan [464]; Kazakhstan; Kenya; Kuwait [464]; Kyrgyzstan; Latvia (ex); Lebanon [630,653]; Lesotho; Libya [528]; Lithuania (ex) [106]; Luxembourg; Malawi [78,452]; Mali; Mauritania [724]; Moldova [106]; Morocco [183]; Mozambique [1017, 1020]; Namibia [1020]; Niger [805,942]; Nigeria [942]; Oman [464]; Pakistan [797,930]; Poland; Portugal; Romania; Russia; Rwanda [322]; Saudi Arabia [464]; Senegal [449,724,942]; Sierra Leone; Somalia [337,367]; South Africa [1020]; Spain [274]; Sudan; Swaziland; Switzerland; Syria [465,630]; Tajikistan; Tanzania; Togo; Tunisia [1173]; Turkey [629, 1 106]; Turkmenia; United Arab Emirates [465]; Ukraine [106]; United Kingdom [242]; [USA: Hog lsland]; Uganda [120]; Uzbekistan; Western Sahara [1113]; Yemen [464]; Yugoslavia; Zaire; Zamhia [75]; Zimbabwe [1020]

Felis termmincki Vigors \& Horsfield 1827 I Id $27,436,550$

Asiatic Golden Cat, Temminck's Cat

Bangladesh [602,603,604,970]; Bhutan; Cambodia; China [994]; India [38,136]; Indonesia: Sumatra [934]; Lao P.D.R. [271,417,837]; Malaysia: Peninsular Malaysia [721]; Myanmar [955]; Nepal [756]; Thailand [96, 151]; Viet Nam $[261,837,853,1025]$

\section{Felis tigrina Schreber 1775}

Little Spotted Cat, Tiger Cat, Oncilla

Argentina [124,699,718,833,915]; Brazil [251]; Colombia [316]; Costa Rica [372,1122]; Ecuador; French Guiana [316]; Guyana [316]; ?Nicaragua; ?Paraguay; ?Peru [53]; Suriname [316,532]; Venezuela [316,448,766]

Felis viverrina Bennett 1833

II $\quad-\quad 436,550$

Fishing Cat

Bangladesh [602,603,604,970]; Bhutan; Cambodia; China; India [38, 136]; Indonesia: Bali, Java, Sumatra; Lao P.D.R. [271,417]; Malaysia: Peninsular Malaysia [1116]; Myanmar [955]; Nepal [756]; Pakistan [797,930]; Sri Lanka; Taiwan; Thailand [96,151]; Viet Nam [837,853,1025] 
Margay, Tree Ocelot

Argentina [124,699,832,833,915]; Belize [442,61 7]; Bolivia [1 198]; Brazil [251]; Colombia [316]; Costa Rica; Ecuador; El Salvador [178]; ?French Guiana; Guatemala [442]; Guyana [316]; Honduras [703]; Mexico [442,906]; Nicaragua; Panama [316,442]; Paraguay [915]; Peru [420]; Suriname [316,532]; USA [442]; Uruguay [915,1190]; Venezuela [316,448]

Felis yagouaroundi E. Geoffroy 1803

Jaguarundi

Appendix I populations $\quad$ ] $\quad$ Id $436,442,1098$

Belize [617]; Costa Rica [1122]; El Salvador [178]; Guatemala [404]; Honduras [703]; Mexico [906]; ?Nicaragua; Panama [316]; USA

\section{Appendix II populations $11 \quad$ Id $184,436,1098$}

Argentina [124,699,701,833,915]; Bolivia [1198]; Brazil [251]; Colombia [316]; Ecuador; French Guiana [316]; Guyana [316]; Paraguay [915]; Peru [420]; Suriname [316,532]; Uruguay [1190]; Venezuela [316,319,448]

Neofelis nebulosa (Griffith 1821) $\quad 1 \quad$ V 27,223,436,550,723

Clouded Leopard

Bangladesh (ex?) $\{393,602,603,604,970]$; Bhutan; Brunei; ?Cambodia; China [994,1 169]; India [38]; 1ndonesia: Kalimantan [1009], Sumatra [961,964]; Lao P.D.R. [271.417.976]; Malaysia: Peninsular Malaysia [721], Sabah [266,468,891], Sarawak [891]; Myanmar [955]; Nepal [284,756]; Taiwan [203,815]; Thailand [96,151]; Viet Nam $[837,853,1025]$

Panthera leo (Linnaeus 1758) $\quad 11 \quad$ 33,436,449,724,941

(except subspecies persica)

Lion

Algeria (ex); Angola [518]; Benin [942,978]; Botswana [1020]; Burkina Faso; Burundi [1 128]; Cameroon [942]; Central African Republic [689]; Chad 1801,942]; Congo [691]; Côte d'Tvoire [942]; Egypt (ex); Ethiopia; Gabon [691]; Gambia [942]; Ghana [942]; Guinea [942]; ?Guinea-Bissau; Kenya [613]; Malawi [78,452, 1018]; Mali [975]; ?Mauritania; Morocco (ex); Mozambique [1017,1020,1089]; Namibia [1020,1036]; Niger [626]; Nigeria [610,942]; ?Rwanda; Senegal [307]; Sierra Leone [942]: Somalia [337,367]; South Africa [748, 1020]; Sudan [503]; Tanzania [613]; Togo [942]; Tunisia (ex); Uganda [120,613]; Western Sahara (ex) |1113]; Zaire [991]; Zambia [75]; Zimbabwe [1020]

$\begin{array}{llll}\text { Panthera leo persica Meyer } 1826 & \text { I } & \text { E } & 27,572\end{array}$

Asiatic Lion

Greece (ex); India [38]; Iran (ex) [646]; Iraq (ex); ]srael (ex) [732]; Pakistan (ex) [930]; Syria (ex) [630]

Panthera onca (Linnaeus 1758)

I $\quad 1065,1098$

\section{Jaguar}

Argentina [124,184,699,701,833,915]; Belize [51,442]; Bolivia [184]; Brazil [184,251]; Colombia [184,316]; Costa Rica [442,1122]; Ecuador; El Salvador (ex) [442]; French Guiana [184,316]; Guatemala [442]; Guyana [184,316]; Honduras [442,703]; Mexico [442,906]; Nicaragua [442]; Panama [3]6,442]; Paraguay [184,915]; Peru [184]; Suriname [184,316,532]; USA [442]; Uruguay (ex); Venezuela [184,316]

Panthera pardus (Linnaeus 1758)

Leopard

Appendix I populations

J $T$

$27,435,436,449,550,553,724$, 733,942

Afghanistan; Algeria; Angola [51 8,784]; Armenia [106]; Azerbaijan [106]; Bangladesh [393,602,603,604,970]; Benin [978]; Bhutan; Burkina Faso [784,944]; Burundi [1128, 1179]; Cambodia; Cameroon [784]; Chad [23]; China [550,994]; Congo [784]; Côte d'lvoire [784,1178]; Djibouti [62]; Egypt (ex?) [835]; Equatorial Gujnea [108]; Gabon [784]; Gambia; Georgia [106]; Ghana [83]; Guinea; ?Guinea-Bissau; Hong Kong (ex) [709]; India [38,99,100]; Indonesia: Bali, Java, Sumatra [934]; 1ran [646]; 1 raq [464,687]l; Istael [464,542,732]; Jordan 
[464]; D.P.R. Korea [1 184]; Korea Republic [1184]; Lao P.D.R. [271,977]; Lebanon (ex?) [630,653]; Lesotho [1020]; Liberia [628]; Libya (ex) [528]; Malaysia: Peninsular Malaysia; Mali [784]; ?Mauritania; Morocco [183]; Myanmar [955]; Namibia [1020]; Nepal [756]; Niger [784,872]; Nigeria [19,784,940]; Oman [464]; Pakistan [797,930]; Russia [106]; ?Rwanda; Saudi Arabia [73,464]; Senegal [307,784]; Sierra Leone [59, 1086]; Singapore (ex) [721]; Somalia [337,367]; Sri Lanka; Sudan [503,996]; ?Swaziland; Syria (ex) $[464,630]$; ?Tajikistan [106]; Thailand [96,151,890]; ?Togo [784]; Tunisia; Turkey [158,629,1106]; Turkmenia [106]; Uganda [120,613]; ?Uzbekistan [106]; United Arab Emirates [464]; Viet Nam [837,853,1025]; Zaire $[784,896,991]$

Appendix I populations (export quotas) $\quad I(\mathrm{eq}) \quad \mathrm{T} \quad 435,436,550,724$

Botswana [1020]; Central African Republic [784]; Ethiopia [169,245]; Kenya [444,542]; Malawi [78,452, 1066]; Mozambique [784,1017,1020,1089]; South Africa [1013,1020,1053]; Tanzania [613,771,1067]; Zambia [75,784]; Zimbabwe [1020]

\section{Panthera tigris (Linnaeus 1758) \\ $1 \quad$ E $27,714,775$}

Tiger

Afghanistan (ex?); Bangladesh [393,602,603,604,970]; Bhutan [292]; Cambodia; China [551,582]; India [38,67,99,100,912]; Indonesia: Java, Sumatra [967]; lran (ex) [646]; Kazakhstan [106]; D.P.R. Korea (ex?) [1184]; Kyrgyzstan [106]; Lao P.D.R. [271,837,976,977]; Malaysia: Peninsular Malaysia [721]; Myanmar [955]; Nepal [756]; Pakistan (ex) [930]; Russia [106,887]; Singapore (ex) [721]; Tajikistan [106]; Thailand $[96,151,890]$; ?Turkey (ex); Turkmenia [106]; Uzbekistan [106]; Viet Nam $[684,837,853,1025$ ]
Panthera uncia (Schreber 1775)
$1 \quad \mathrm{E}$
$27,142,550$

Snow Leopard, Ounce

Afghanistan [793]; Bhutan [387]; China [669,981,985,994]; India [38,413,836]; Mongolia [695]; Nepal $[552,756]$; Pakistan $[694,797,930]$; Russia [106]

\section{Order PINNIPEDIA}

\section{Family OTARIIDAE}

Arctocephalus australis (Zimmermann 1783)

II

923,987

South American Fur Seal, Southern Fur Seal

Argentina [124,184,915]; Brazil [251]; Chile [394,746,915]; Falkland Islands [184,915]; Peru [85]; Uruguay $[184,915,1123,1190]$

Arctocephalus forsteri (Lesson 1828)

II

923,987

New Zealand Fur Seal

Australia; New Zealand [1083]

Arctocephalus galapagoensis Heller 1904

II

$923,987,1098$

Galapagos Islands Fur Seal

Galapagos Islands

Arctocephalus gazella (Peters 1875)

II

923,987

Antarctic Fur Seal, Kerguelen Fur Seal

Bouvet Island [610,1020]; Brazil [200]; Chile [915]; Falkland Islands: South Georgia, South Orkney Islands, South Sandwich Islands [610], South Shetland Islands [610]; French Southern and Antarctic Territories: Crozet Islands [1020], Kerguelen Islands [610,1020]; Heard and Macdonald Islands [596]; South Africa: Marion Island, Prince Edward Island [1020] 
Arctocephalus pusillus (Schreber 1776)

II

923,987

(includes $A$. doriferus, A. tasmanicus)

Afro-Australian Fur Seal, South African Fur Seal, Cape Fur Seal

Angola [1020]; Australia [850]; Mozambique [1017]; Namibia [1020]; South Africa: including Marion Island $[599,1020]$

Arctocephalus townsendi Merriam 1897

Guadalupe Fur Seal

Mexico [442]; USA [442]

Arctocephalus tropicalis (Gray 1872)

II

I V $\quad 923,987,1098$

(includes A. elegans)

Sub-antarctic Fur Seal, Amsterdam Island Fur Seal

Argentina; Australia: Macquarie Island [610]; Brazil [610]; Chile [394,1103]; Falkland Islands: South Georgia [610]; French Southern and Antarctic Territories: Amsterdam Island [610], Crozet Islands [585,610], Saint Pau]; New Zealand [610]; Saint Helena: Gough Island, Inaccessible, Nightingale, Tristan da Cunha Islands [610]; South Africa: including Marion Island [610], Prince Edward Island [600]
Zalophus californianus japonicus (Peters 1866)
Ex?
$27,923,987$

Japanese Sealion

Japan (ex?); D.P.R. Korea (ex?) [1184]; Korea Republic (ex?) [1184]

Family ODOBENIDAE

Odobenus rosmarus (Linnaeus 1758)

III CA -

$442,918,923,987$

(except subspecies laptevi)

Walrus

Canada; Denmark; Finland; France [299]; Greenland; Iceland; Ireland; Japan; Netherlands; Norway; Russia:

Franz Josef Land [106]; Svalbard and Jan Mayen Islands; Sweden; United Kingdom; USA

Odobenus rosmarus laptevi Chapskii 1940

III CA $\mathrm{K} \quad 27, \mathrm{I06}$

\section{Laptev Walrus}

Russia [106]

\section{Family PHOCIDAE}

Mirounga leonina (Linnaeus 1758)

II

I $99,923,987$

Southern Elephant-seal

Angola [1020]; Argentina [184,610,915]; Macquarie Island [610]; Bouvet Island [610]; British Antarctic Territory: South Orkney Islands [610], South Shetland Islands [610]; Brazil [200]; Chile: Juan Fernandez Archipelago [184,394,610,746,915]; Falkland Islands: including South Sandwich Islands, South Georgia [184,610,915]; French Southern and Antarctic Territories: Amsterdam Island, Crozet Islands, Kerguelen Islands [610]; Heard Island [596,610]; Namibia [1020]; New Zealand: Campbell Island [610]; Saint Helena: Gough Island, Tristan da Cunha Islands [610]; South Africa: Marion Island, Prince Edward Island [234,235,1020]; Uruguay [915, 1190$]$

Monachus monachus (Hermann 1779)

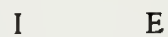

$27,923,987$

Mediterranean Monk Seal

Albania (ex?); Algeria [995]; Bulgaria [121]; Canary Islands (ex?) [995]; Cyprus [995]; Egypt (ex) [995]; France (ex): including Corsica (ex?) [340,995]; Georgia (ex?) [106]; Greece [995]; Israel (ex) [995]; Italy: including Sardinia (ex?), Sicily (ex?) [995]; Lebanon [630,653,995]; Libya [528]; Madeira; Malta; Mauritania [995]; Morocco [183]; Portugal [995]; Romania; Russia (ex?) [106]; Spain (ex): including Balearic Islands (ex?) [995]; Syria (ex?) [630]; Tunisia [995]; Turkey [629,995,1106]; Ukraine (ex?) [106]; Western Sahara [971,1113]; Yugoslavia 
Monachus schauinslandi Matschie 1905 Hawaiian Monk Seal

Hawaiian Islands
1

E

$923,987,1098$

1

E

$923,987,1098$

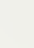

Monachus tropicalis (Gray 1850)

\author{
923,987,1098
}

Bahamas (ex?) [442]; Colombia: Isla de Providencia (ex) [316]; Cuba (ex); Guadeloupe (ex); Haiti: Alta Vela (ex); Honduras (ex) [703]; Jamaica: Pedro Cays (ex); Mexico (ex?) [442]; USA (ex) [442]
Phoca hispida saimensis Nordquist 1899
(= Pusa hispida saimenris)
Saimaa Ringed Seal
Finland

Phoca vitulina stejnegeri J. Allen 1902

V

150

Kuril Seal

Kuril Sea

\title{
Order PROBOSCIDEA
}

\section{Family ELEPHANTIDAE}

\section{Elephas maximus Linnaeus 1758}

1

E

$27,923,987,1041$

Indian Elephant, Asian Elephant

Bangladesh [393,602,603,604,970]; Bhutan; Brunei; Cambodia; China [100,226,439,998, 1056]; India [99]; Indonesia: Kalimantan, Sumatra [792]; Lao P.D.R. [271,417,976]; Malaysia: Peninsular Malaysia [541,607], Sabah [266]; Myanmar [955]; Nepal [756]; Sri Lanka; Thailand [96,151,289]; Viet Nam [684,853, 1025]

Loxodonta africana (Blumenbach 1797)

1 V

$27,294,295,724,845$

\section{African Elephant}

Angola [518,549]; Benin [549,978,1131]; Botswana [549,1020,1033]; Burkina Faso [548,944,1032]; Burundi (ex) [1128,1179]; Cameroon [549,557]; Central African Republic [195,339,549]; Chad [549]; Congo [339,549]; Côte d'Ivoire [549,740]; Equatorial Guinea [108]; Ethiopia [641,724]; Gabon [549]; Gambia (ex); Ghana [549]; Guinea; Guinea-Bissau (ex); Kenya [549,1100]; Lesotho (ex); Liberia [14,549,628]; Malawi [78,452,549,1066]; Mali; Mauritania [549]; Mozambique [549,1017,1020]; Namibia [549,1020]; Niger [549,975]; Nigeria [19,549,940]; Rwanda [549]; Senegal [549]; Sierra Leone [263,549]; Somalia [337,367,549]; South Africa [549,1013, 1020]; Sudan [549,996]; Swaziland (ex); Tanzania [549, 1067]; Togo [549]; Uganda [120,549]; Zaire [549,896,991]; Zambia [75,549]; Zimbabwe [549,1020]

\section{Order HYRACOIDEA}

\section{Family PROCAVIIDAE}

Dendrohyrax validus True 1890 K $\quad 724$

Eastern Tree Dassie

Tanzania: including Pemba 1sland, Zanzibar 


\section{Order SIRENIA}

Family DUGONGIDAE

Dugong dugon (P.L.S. Müller 1776)

Dugong
Appendix I populations
I V $\quad 723,1098$

Bahrain [369]; Bangladesh [602]; Brunei; Cambodia; China; Comoros; Djibouti [932]; Egypt; Ethiopia; Federated States of Micronesia: Yap; Guam; India [38]; Indonesia [934]; Iran; Japan: Ryukyu Islands; Kenya [657]; D.P.R. Korea; Korea Republic; Madagascar [125]; Malaysia: Peninsular Malaysia, Sabah, Sarawak; Maldives (ex); Mauritius; Mozambique [1017,1020]; Myanmar [955,1110]; New Caledonia; Oman; ?Pakistan; Palau; Papua New Guinea; Philippines; Saudi Arabia; Singapore [509]; Solomon Islands; Somalia [125,337]; South Africa [1020]; Sri Lanka [437,960]; Taiwan; Tanzania [611]; Thailand [96,151]; United Arab Emirates; Vanuatu [266,708]; Viet Nam [684,1025]; Yemen

$\begin{array}{llll}\begin{array}{l}\text { Appendix II populations } \\ \text { Australia }\end{array} & \text { II } & \text { V } & 117 \\ \text { Hydrodamalis gigas (Zimmermann 1780) } & - & & \\ \end{array}$

\section{Steller's Sea Cow}

Bering Sea (ex); North Pacific Ocean (ex)

Family TRICHECH1DAE

Trichechus inunguis (Natterer 1883)

I $\quad$ V 1098

Amazonian Manatee, South American Manatee

Brazil [251,290]; Colombia [316]; Ecuador; Peru; Venezuela [316]

Trichechus manatus Linnaeus 1758

I V

1098

Caribbean Manatee, North American Manatee, American Manatee

Antigua (ex) and Barbuda [1119]; Bahamas [442]; Belize [442]; Brazil [251]; British Virgin Islands (ex) [1119]; Colombia [316]; Costa Rica [442]; Cuba [330,442]; Dominican Republic [442]; French Guiana; Guadeloupe (ex) [1119]; Guatemala; Guyana [316]; Haiti [442]; Honduras [442,703,911]; Jamaica [442]; Martinique (ex) [11 19]; Mexico [188,442,1133]; Netherlands Antilles: Curacao; Nicaragua [442]; Panama [442,980]; Puerto Rico [442,883]; Suriname [316,532]; Trinidad and Tobago [13,442]; USA [442,474,616]; Venezuela [246,316]; Virgin Islands of the United States (ex) [1119]

Trichechus senegalensis Link 1795

II $\quad$ V $\quad 27,724$

African Manatee

Angola [477,518]; Benin (ex?) [978,1131]; Cameroon [557]; Chad; Congo [1031]; Côte d'Ivoire [813]; Equatorial Guinea [108]; Gabon [691,813]; Gambia; Ghana [189]; ?Guinea; ?Guinea-Bissau; Liberia [14,628,1130]; Mali [975]; Mauritania [303]; Niger [813]; Nigeria [940]; Senegal [813]; Sierra Leone [481]; Togo [813]; Zaire [813]

\section{Order PERISSODACTYLA}

\section{Family EQUIDAE}

Equus africanus (Fitzinger 1857)

I $\quad$ E $27,229,724$

(=E. asinus Linnaeus 1758)

African Ass, African Wild Ass

[Australia]; Chad; [Galapagos Islands]; Egypt (ex) [32]; Ethiopia [619]; Somalia [337,367]; Sudan [1205, 1206]; [USA] 
Equus grevyi Oustalet 1882

1

E

27

Grevy's Zebra

Ethiopia [149]; Kenya [288]; Somalia (ex) [367]; Sudan?

Equus hemionus Pallas 1775

(except subspecies hemionus, hemippus, khur)

(includes E. kiang Moorcroft 1841, E. kulan, E. onager Boddaert 1785)

Asiatic Wild Ass, Onager Ass, Kulan, Kiang

Afghanistan [440]; China [994]; India [38,836]; Iran [646]; [Israel [230]]; Kazakhstan [106]; Kyrgyzstan [106];

Tajikistan [106]; Turkmenia [27]

Equus hemionus hemionus Pallas 1775

l V 27,1024

(includes E. luteus Matschie 1911)

Mongolian Wild Ass, Dziggetai

China; Kazakhstan (ex?) [27,424]; Mongolia [695]; [Uzbekistan: Barsahelmus Island [104]]

Equus hemionus hemippus 1 1. Geoffroy $1855 \quad 11 \quad$ Ex $\quad 27$

(=E. onager hemippus)

Syrian Wild Ass, Achdari

Iran (ex); Iraq (ex); 1srael (ex); Saudi Arabia (ex); Syria (ex) [630]

Equus hemionus khur Lesson 1827

$1 \quad \mathrm{E}$

$27,38,556,633$

(=E. onager khur)

Indian Wild Ass, Khur

India; ?Iran; Pakistan

Equus przewalskii Poliakov 1881

I Ex? 27,1024

(=E. caballus prewalskii, E. ferus prewalskii)

Przewalski's Horse, Takh

China (ex?); Mongolia (ex?)

Equus quagga Gmelin 1788

Quagga

South Africa (ex)

Equus zebra hartmannae Matschie 1898

$11 \quad$ V

27,724

Hartmann's Mountain Zebra

?Angola [518,583]; Namibia [1020]; South Africa [1013]

Equus zebra zebra Linnaeus 1758

$1 \quad E$

$27,724,1013,1020$

Cape Mountain Zebra

South Africa

\section{Family TAPIRIDAE}

Tapirus bairdii (Gill 1865)

Central American Tapir, Baird's Tapir

Ex $\quad 1014$

Belize [617,716]; Colombia [316]; Costa Rica; Ecuador; El Salvador (ex); Guatemala [404]; Honduras [703];

Mexico (ex?) [906]; Nicaragua; Panama [316]

Tapirus indicus Desmarest 1819

$1 \quad \mathrm{E}$

27

Malayan Tapir

Indonesia: Sumatra [934,965]; Lao P.D.R. [271,277,976]; Malaysia: Peninsular Malaysia; Myanmar [955];

Thailand [96,151]; Viet Nam [1025,1158] 
( $=T$. roulini)

Mountain Tapir, Woolly Tapir, Andean Tapir

Colombia [316]; Ecuador; Peru [85,420]; Venezuela [1076]

Tapirus terrestris (Linnaeus 1758)

$184,185,492$

Brazilian Tapir, South American Tapir, Lowland Tapir

Argentina [124,699,701,833,915]; Bolivia [1198]; Brazil [251,744]; Colombia [316]; Ecuador [1076]; French Guiana; Guyana [24,316]; Paraguay [915]; Peru [85,420]; Suriname [316,532]; Venezuela [316,448,1076]

\section{Family RHINOCEROTIDAE}

Ceratotherium simum simum (Burchell 1817) I $368,724,939,1157$

Southern White Rhinoceros, Southern Square-lipped Rhinoceros

[Angola (ex) [518]]; [Botswana]; [Kenya]; [Mozambique [1017]]; [Namibia]; South Africa [1020]; [Swaziland]; [Zambia]; [Zimbabwe]

$\begin{array}{llll}\text { Ceratotherium simum cottoni (Lydekker 1908) } \quad \text { I } & \text { E } & 27,368,505,724,939,1004,1157\end{array}$

Northern White Rhinoceros, Northern Square-lipped Rhinoceros

Central African Republic; Chad (ex); Sudan [503]; Uganda [120]; Zaire

$\begin{array}{llll}\text { Dicerorhinus sumatrensis (Fischer 1814) } & \text { I } & \text { E } & 27,939\end{array}$

(= Didermocerus sumatrensis)

\section{Sumatran Rhinoceros}

Bangladesh (ex) [602]; Brunei (ex); ?Cambodia; India (ex?); Indonesia: Kalimantan, Sumatra [934]; Lao P.D.R. (ex?) $[271,417,976]$; Malaysia: Peninsular Malaysia [356], Sabah [266], Sarawak (ex); Myanmar [955]; Thailand [96,151]; Viet Nam [684,853,1025]

Diceros bicornis (Linnaeus 1758)

$$
1 \quad \text { E } 27,368,724,939,1096,1157
$$

Black Rhinoceros, Hook-lipped Rhinoceros

Angola [161]; Botswana [161]; Burundi (ex) [1128]; Cameroon; Central African Republic; Chad; Congo (ex); Côte d'lvoire (ex); Ethiopia [641]; Kenya [166,287]; Malawi [78,452,547,1066]; Mozambique [1017,1020]; Namibia [1020]; Niger (ex); Nigeria (ex) [19,940]; Rwanda; Somalia [337,367]; South Africa [1013,1020]; Sudan [503]; Tanzania; Uganda; Zaire (ex); Zambia [75,1174]; Zimbabwe [1020]

$\begin{array}{llll}\text { Rhinoceros sondaicus Desmarest } 1822 & \text { I } & \text { E } & 27,939\end{array}$

Javan Rhinoceros

Bangladesh (ex) [602]; Cambodia (ex?) [1099]; ?China (ex); India (ex); Indonesia: Java [966], Sumatra (ex); Lao P.D.R. (ex?) (271,417,976]; Malaysia: Peninsular Malaysia [48]; Myanmar (ex?) [955]; Thailand (ex?) [96, 15I]; Viet Nam (ex) $[684,853,963,1025]$

Rhinoceros unicornis Linnaeus 1758 $1 \quad \mathrm{E}$ $27,225,644,939$

Great Indian Rhinoceros

Bangladesh (ex) [602,970]; Bhutan [711]; India [38,711]; Nepal [111,756]; Pakistan (ex) [930]

\section{Order ARTIODACTYLA}

Family SUIDAE

Babyrousa babyrussa (Linnaeus 1758) I $\quad$ V 27

Babirusa

Indonesia: Buru, Sula Islands, Sulawesi, Togian Islands 
Ryukyu Islands Wild Pig

Japan: Ryukyu lslands

Sus salvanius (Hodgson 1847)

Pygmy Hog

Bangladesh (ex); Bhutan (ex?); India [38]; Nepal (ex?) [756]

Sus verrucosus Mūller \& Schlegel 1847

V

913,1118

Javan Warty Pig

Indonesia: Bawean Island, Java

\section{Family TAYASSUIDAE}

Catagonus wagneri (Rusconi 1930)

1

1029,1098

Chacoan Peccary, Tagua

Argentina [124,699,701,833,915]; Bolivia [321]; Paraguay [915]

Tayassu pecari (Link 1795)

II

$185,700,1029$

White-lipped Peccary

Argentina [915]; Belize; Bolivia [1198]; Brazi]; Colombia [316]; Costa Rica; Ecuador; El Salvador; French Guiana (France); Guatemala; Guyana [316]; Honduras [703]; Mexico [906]; Nicaragua; Panama [316]; Paraguay [915]; Peru; Suriname [316]; Venezuela [316]
Tayassu tajacu (Linnaeus 1758)
11
$185,700,1029$

Collared Peccary, Javelina

Argentina [699,701,833,915]; Belize [442,617]; Bolivia [1198]; Brazil [196,251,744,869]; Colombia [316]; Costa Rica [442,728]; [Cuba [1119,1120]]; Ecuador; El Salvador [178,442]; French Guiana [316]; Guatemala [404,442]; Guyana [24,316,943]; Honduras [442,703]; Mexico [442,906]; Nicaragua [442]; Panama [442]; Paraguay [915,1129]; Peru [85,420]; Suriname [316,532]; Trinidad and Tobago [13]; Uruguay [958]; Venezue]a $[316,319,448,766,1201]$

(Non-CITES populations in USA [442])

\section{Family H1PPOPOTAMIDAE}

Choeropsis liberiensis (Morton 1844)

I1 V $27,724,1004$

Pygmy Hippopotamus

Côte d'Ivoire [768,1178]; Guinea [935]; ?Guinea-Bissau [237]; Liberia [14]; Nigeria (ex) [19,237]; Sierra Leone $[263,1085]$
Hippopotamus amphibius Linnaeus 1758
III $\mathrm{GH}$
724,1004

Hippopotamus

Algeria (ex); Angola [518]; Benin [978,1131]; Botswana [1020]; Burkina Faso [43]; Burundi [1128,1179]; ?Cameroon; Central African Republic [689]; Chad [689]; Congo [1031]; Côte d'lvoire; Egypt (ex) [835]; Equatorial Guinea [108,182]; Ethiopia; Gabon [691]; Gambia; Ghana [189]; Guinea-Bissau [1004]; Kenya [593]; Liberia [628,1130]; Malawi [78,1066]; Mali [975]; Mozambique [1017,1020]; Namibia [1020]; Niger [418]; Nigeria [20,859]; Rwanda [769]; Senegal [307]; Sierra Leone [458,1086]; Somalia [337,367]; South Africa [1013,1020]; Sudan [503]; Tanzania [771]; ?Togo; Uganda [33,120]; Zaire; Zambia; Zimbabwe [1020] 


\section{Family CAMELIDAE}

Camelus bactrianus Linnaeus 1758

(=C. ferus Przewalski 1883)

Wild Bactrian Camel

China; Mongolia [695]

Lama guanicoe (P.L.S. Mūller 1776)

I1

185,700

\section{Guanaco}

Argentina [124,699,701,833,915]; Bolivia [1198]; Chile [394,696,746,870,915,1069]; Paraguay [915,1129]; Peru $[420,851]$

Vicugna vicugna (Molina 1782)

Vicuña
Appendix I populations
I V 185,1098

Argentina [124,699,701,833,915]; Bolivia [1198]; Chile: all populations except part of the population of Parinacota Province la. region of Tarapacá [203,394,746,915]; Peru: all populations except populations of Pampa Galeras National Reserve and Nuclear Zone, Pedregal, Oscconta and Sawacocha (Province of Lucanas), Sais Picotani (Province of Azangaro), Sais Tupac Amaru (Province of Junín), and of Salinas Aguada Blanca National Reserve (Provinces of Arequipa and Cailloma)

Appendix II populations II $\quad$ V 185,1098

Chile: part of the populations of Parinacota Province Ia. region of Tarapaca [203,394,746,915]; Peru: populations of Pampa Galeras National Reserve and Nuclear Zone, Pedregal, Oscconta and Sawacocha (Province of Lucanas), Sais Picotani (Province of Azangaro), Sais Tupac Amaru (Province of Junín), and of Salinas Aguada Blanca National Reserve (Provinces of Arequipa and Cailloma)

Family TRAGULIDAE

Hyemoschus aquaticus (Ogilby 1841)

III GH -

724

Water Chevrotain

Angola [518]; Benin; ?Burkina Faso; Cameroon; Central African Republic [689]; Congo [1031]; Côte d'Ivoire [1178]; Equatorial Guinea [182]; Gabon [691]; Ghana [189]; Guinea [937]; ?Guinea-Bissau; Liberia [14,628]; Nigeria [20,859]; Senegal [1071]; Sierra Leone; ?Togo; Uganda [120]; Zaire [896,991]

\section{Family CERVIDAE}

Blastocerus dichotomus (Illiger 1815)

$$
\text { I V } 247,1098,1166
$$

Marsh Deer, Guasu Pucu

Argentina [124,699,701,915]; Bolivia; Brazil [41]; Paraguay [915]; Peru; Uruguay (ex) $[915,958,1190]$

Cervus albirostris Przewalski 1883

V $\quad 27,247,1166$

Thorold's Deer, White-lipped Deer

China

Cervus dama mesopotamicus (Brooke 1875)

1

E

$27,247,275,1166$

(= Dama mesopotamica)

Persian Fallow Deer

Iran; Iraq (ex?) [464]; Israel (ex); Jordan (ex) [464]; Lebanon (ex) [464]

Cervus duvauceli G. Cuvier 1823

I E

$27,247,843,979,1010,1166$

Swamp Deer, Barasingha

Bangladesh (ex) [602,603,604,970]; India [38,959]; Nepal [756]; Pakistan (ex) [930]

Cervus elaphus bactrianus Lydekker 1900

II $\quad$ E

$27,105,247,1166$

Bactrian Deer, Bokharan Deer, Bactrian Red Deer

Afghanistan; Kazakhstan [106]; Tajikistan [106]; Turkmenia [106]; Uzbekistan [106] 
France: Corsica (ex); Italy: Sardinia

Cervus elaphus hanglu Wagner 1844

Hangul, Kashmir Stag

India [38]; Pakistan (ex?) [930]

Cervus elaphus macneilli Lydekker 1909

Id $27,247,1166$

(=C. canadensis macneilli)

\section{MacNeill's Red Deer}

China

Cervus elaphus wallichi G. Cuvier 1823

Shou

Bhutan (ex?); China

Cervus elaphus yarkandensis Blanford 1892

E

Yarkand Deer

China

Cervus eldi McClelland 1842

(except subspecies eldi, siamensis)

E

$27,247,1166$

Thamin

Myanmar $[955,957]$; Thailand

Cervus eldi eldi McClelland 1842

Manipur Brow-antlered Deer, Sangai

India

Cervus eldi siamensis Lydekker 1915

Thailand Brow-antlered Deer

Cambodia [1099]; China (ex?) [1 169]; Lao P.D.R. [271,976,977]; Thailand [96,151]; Viet Nam [684,853, 1025]

Cervus nippon grassianus (Heude 1884)

Ex? $\quad 27,247,343,1166$

Shansi Sika

China (ex?)

Cervus nippon keramae (Kuroda 1924)

E $27,247,343,1166$

Ryukyu Sika, Kerama Deer

Japan: Ryukyu Islands

Cervus nippon mandarinus Milne-Edwards 1871

E

North China Sika

China: Hebei [814]

Cervus nippon pseudaxis Eydoux \& Souleyet 1841

(=C.n. kopschi Swinhoe 1873)

South China Sika

China: ?Guangdong, Guangxi, Jiangxi, Zhejiang [814]; Viet Nam [1166] 
Cervus nippon sichanicus Guo, Chen \& Wang 1978

Sichuan Sika

$1 \quad 814$

China: Gansu, Sichuan

Cervus nippon taiouanus Blyth 1860

Formosan Sika

[Japan: Oshima 1sland]; Taiwan (ex?)

Cervus porcinus annamiticus Heude 1888

(= Axis porcinus annamiticus)

Indochinese Hog Deer

Cambodia; Indonesia; Lao P.D.R. [271,417]; Thailand [96,151]; Viet Nam [837,1025]

Cervus porcinus calamianensis Heude 1888

I V $\quad 27,247,1166$

(= Axis calamianensis)

Calamian Hog Deer

Philippines: Calamian 1slands

Cervus porcinus kuhli (Müller \& Schlegel 1844)

(= Axis kuhli)

Kuhl's Hog Deer

Indonesia: Bawean Island

Cervus unicolor alfredi Sclater 1876

(=C. alfredi)

Visayan Spotted Deer

Philippines: Visayan Islands

Elaphurus davidianus Milne-Edwards $1866 \quad$ E $\quad$ E 1166,814

Père David's Deer

China (ex) [814]

Hippocamelus antisensis (d'Orbigny 1834)

$1 \quad \mathrm{~V} \quad 185,247,1098$

North Andean Huemul, Peruvian Huemul, Taruca

Argentina [124,701,833,915]; Bolivia [1198]; Chile [394,746,915]; Peru [420]

Hippocamelus bisulcus (Molina 1782)

I E $185,247,1098$

South Andean Huemul, Chilean Huemul

Argentina [124,833,915, 1166]; Chile [394,746,882,915,1166]

Hydropotes inermis Swinhoe 1870

$\mathbf{R}$

1166

Chinese Water Deer

China; [France]; D.P.R. Korea; Korea Republic; [United Kingdom]

Mazama americana cerasina Hollister 1914

$111 \mathrm{GT}$

442

Middle American Red Brocket

Belize [617]; Costa Rica [442]; El Salvador [442]; Guatemala [404,442]; Honduras [442,703]; Nicaragua [442];

Panama [442]

Moschus berezovskii Flerov 1929

11

(=M. chrysogaster berezovskii)

Dwarf Musk Deer, South China Forest Musk Deer

China; Lao P.D.R. [271]; Viet Nam $[259,684,1025]$ 
Moschus chrysogaster (Hodgson 1839)

(= M. moschiferus moschiferus sensu Flerov 1952, pro parte)

Himalayan Forest Musk Deer

Appendix I populations

1

$3,27,247,411,415,432$

Bhutan; India [38]; Nepal [666,756]

Appendix II populations

II

$27,247,411,415,425,432$

China [186]

Moschus fuscus Li 1981

II

814

(= M. chrysogaster fuscus)

China: Xizang, Yunnan [814]

Moschus moschiferus Linnaeus 1758

II

425,1144

(= M. sibiricus Pallas 1779)

(includes $M$. anhuiensis Wang, Hu \& Yan 1982)

Siberian Musk Deer

China; D.P.R. Korea [814,1184]; Korea Republic [1184]; Mongolia [695]; Russia [106]

Moschus sifanicus (Büchner 1891)

(= M. moschiferus moschiferus sensu Flerov 1952, pro parte)

Alpine Musk Deer

Appendix I populations

1

$27,247,415,425,432$

Afghanistan; Bhutan; India [38]; Myanmar [955]; Nepal; Pakistan [797,930]

Appendix II populations

II

$27,247,415,425,432$

China

Muntiacus crinifrons (Sclater 1885)

1

V

$247,668,1000$

Black Muntjac

China

Muntiacus feai (Thomas \& Doria 1889)

E

$27,247,447$

Fea's Muntjac

China [667]; Myanmar [955]; [Thailand [96,151]]

Odocoileus hemionus cerrosensis Merriam 1898

Cedros Island Mule Deer, Cedros Island Black-tailed Deer

R

$247,906,1098$

Mexico: Cedros Island

Odocoileus virginianus clavium Barbour \& Allan 1922 - $\quad$ R 1098

Key Deer Toy Deer

USA: Florida - islands near Big Pine Key

Odocoileus virginianus mayensis

III GT

(subspecies could not be traced, no information available)

?Guatemala

Ozotoceros bezoarticus (Linnaeus 1758)

1

185

(= Odocoileus bezoarcticus)

(except subspecies celer)

Pampas Deer

Argentina [124,699,701,833,915]; Bolivia; Brazil [251]; Paraguay [915]; Uruguay [79,915] 
Ozotoceros bezoarticus celer Cabrera 1943

(= Odocoileus bezoarcticus celer)

$1 \quad$ E 247,1098

Argentinian Pampas Deer, Venado, Gama

Argentina

Pudu mephistophiles de Winton 1896

II

Id

$185,495,1098,1166$

Northern Pudu

Colombia [316]; Ecuador; Peru [420]

Pudu pudu (Molina 1782)

Southern Pudu

Argentina [833,915]; Chile $[394,746,870,915,1069]$

Family BOVIDAE

Addax nasomaculatus (Blainville 1816)

I $\quad$ E 27,636

Addax

Algeria [391,1015]; Chad [803]; Egypt (ex) [355]; Libya [391,528]; Mali; Mauritania [303,1104]; Niger [391,802,805]; Sudan [639]; Tunisia (ex?) [391]; Western Sahara (ex?) [1113]

Aepyceros melampus petersi Bocage 1879

E $\quad 27,724$

Black-faced Impala

Angola [518]; Namibia [1020]

Alcelaphus buselaphus swaynei (Sclater 1892)

E

$27,652,724$

Swayne's Hartebeest

Ethiopia [245]; ?Somalia [367]

Alcelaphus buselaphus tora (Gray 1873)

Tora Hartebeest

Egypt (ex); Ethiopia [1004]; Sudan

Ammodorcas clarkei (Thomas 1891)

Dibatag, Clarke's Gazelle

Ethiopia; Somalia $[337,367,1007]$

Ammotragus lervia (Pallas 1777)

II $\quad$ V 408

Barbary Sheep, Aoudad, Uaddan

Algeria [239]; Chad [276,689]; Egypt [835]; ?Israel (ex); Libya [239,528]; Mali [975]; Mauritania (ex?); Morocco (ex?) [183]; Niger [805]; Sudan [239,1175]; Tunisia (ex?) [239]; [USA]; Western Sahara (ex) $[804,1113]$

Antilocapra americana (Ord 1815)

I $\quad-\quad 693,817,906$

(except subspecies peninsularis, sonoriensis)

Mexican Pronghorn

Mexico

Antilocapra americana peninsularis (Nelson 1912)

I

E

906,1098

Baja California Pronghorn

Mexico

Antilocapra americana sonoriensis (Goldman 1945)

Sonoran Pronghorn

Mexico [442,906]

I E 1098,906

(Non-CITES populations in USA [442]) 
Ansilope cervicapra (Linnaeus 1758)

111 NP

244

Blackbuck

[Argentina]; Bangladesh [970]; India [38]; Nepal [756]; Pakistan [930]; [USA]

$\begin{array}{llll}\text { Bison bison athabascae Rhoads I898 } & \text { I } & - & 1097\end{array}$

Wood Bison

Canada; USA (ex) [442]

Bison bonasus (Linnaeus 1758)

V $\quad 27,1097$

(= B. bison bonasus)

European Bison, Wisent

[Belarus [106]]; [Lithuania [106]]; [Moldova [106]]; [Poland]; [Romania]; [Russia [106,258]]; Slovenia (ex)

[842]; Sweden (ex); [Ukraine [106]]

Boocercus eurycerus (Ogilby 1857) III GH - $\quad 724,902$

(= Tragelaphus eurycerus)

Bongo

Angola [518]; Benin (ex?) [978]; ?Burkina Faso ; Cameroon [557]; Central African Republic [194]; Congo [1031]; Côte d'Ivoire [768,1178]; Equatorial Guinea [182]; ?Ethiopia; Gabon [691]; Ghana [560]; Guinea; Guinea-Bissau (ex?); Kenya [33]; Liberia [14,628]; Mali (ex?); Niger (ex?); Sierra Leone [263,481]; Sudan [503,504,651]; Togo; ?Uganda [33,120]; Zaire [896]

Bos gaurus H. Smith 1827

I $\quad$ V 27,1097

(=B. frontalis Lambert 1804)

Gaur, Indian Bison

Bangladesh [393,603,604,970]; Bhutan; Cambodia; China; India [38,99,100]; Lao P.D.R. [271,976,977]; Malaysia: Peninsular Malaysia [236]; Myanmar [955]; Nepal [756]; Thailand [151]; Viet Nam $[684,837,853,1025]$

$\begin{array}{llll}\text { Bos javanicus D'Alton } 1823 & \text { - } & \text { V } & 27,723,1097\end{array}$

(= B. banteng Wagner 1844)

Banteng, Tembadau

Bangladesh (ex?) [602]; Brunei (ex); Cambodia; India (ex); Indonesia: Bali, Java [81,82], Kalimantan; Lao P.D.R. [271,417,837,976]; Malaysia: Peninsular Malaysia (ex?), Sabah [266], Sarawak (ex); Myanmar [955]; Thailand [96,151]; Viet Nam $[684,837,853,1025]$

Bos mutus Przewalski 1883

I $\quad$ E 27,1097

(=B. grunniens Linnaeus 1766)

Wild Yak

?Afghanistan; ?Bhutan; China [42,994]; India [38]; Nepal [756,842]

$\begin{array}{llll}\text { Bos sauveli Urbain } 1937 & \text { I } & \text { E } & 27,1097\end{array}$

(= Novibos sauveli)

Kouprey

Cambodia [685,1099]; Lao P.D.R. [271,417,685,855]; Thailand (ex?) [44,151]; Viet Nam $[684,685,853,1025]$

Bubalus arnee (Kerr 1792) III NP E 27,1097

(excluding the domestic form B. bubalis (Linnaeus 1758))

Wild Asiatic Buffalo

Bangladesh (ex) [602,970]; India [38]; Nepal [756]

Bubalus depressicornis (H. Smith 1827)

I $\quad$ E 27,1097

(= Anoa depressicornis)

Lowland Anoa

Indonesia: Sulawesi 
Bubalus mindorensis (Heude 1888)

I E 27,1097

(= Anoa mindorensis)

\section{Tamaraw}

Philippines: Mindoro

Bubalus quarlesi (Ouwens 1910)

I

E 27,1097

(= Anoa quarlesi)

Mountain Anoa

Indonesia: Sulawesi

Budorcas taxicolor Hodgson 1850

II

798

(except subspecies bedfordi, tibetana)

Takin

Bhutan; China; India [38]; Myanmar

Budorcas taxicolor bedfordi Thomas 1911

II

$\mathbf{R}$

27

Golden Takin

China

Budorcas taxicolor tibetana Milne-Edwards 1874

Sichuan Takin

China

Capra falconeri (Wagner 1839)

$1 \quad \mathrm{~V}$

27

(except subspecies jerdoni, megaceros)

Markhor

Afghanistan [795]; India [38]; Pakistan [12,930]; ?Turkmenia [106]; Tajikistan [106,239]; Uzbekistan [106,239]

Capra falconeri jerdoni Hume 1875

Straight-horned Markhor

II Id $\quad 27,994$

Pakistan

Capra falconeri megaceras Hutton 1842

Kabul Markhor

Afghanistan [440]; Pakistan [930]

Capra ibex walie (Rüppell 1835)

E $\quad 27,812$

(= Capra walie)

Walia Ibex

Ethiopia

Capra pyrenaica pyrenaica Schinz 1838
1
E
$27,797,930$

Pyrenean Ibex

Spain

Capricornis crispus swinhaei Gray 1862

Formosan Serow

Taiwan

Capricornis sumatraensis (Bechstein 1799)

I

E $27,797,930$

(except subspecies sumatraensis)

\section{Mainland Serow}

Bangladesh [393,602,603,604,970]; ?Cambodia; China [994]; India [381]; Lao P.D.R. [271,417,837]; Malaysia; Myanmar [955]; Nepal [666,756]; Thailand [151]; Viet Nam [837,853,1025] 
Capricornis sumatraensis sumatraensis (Bechstein 1799) I Sumatran Serow

Indonesia: Sumatra

Cephalophus adersi Thomas 1918

Aders' Duiker

Kenya [309]; Tanzania: Zanzibar [309]

Cephalophus dorsalis Gray 1846

Bay Duiker

Angola [518]; Benin; Burkina Faso [944]; Cameroon; ?Central African Republic; Congo [691]; Côte d'Ivoire [768,1178]; Equatorial Guinea [182]; Gabon [691]; Ghana [189]; Guinea [937]; ?Guinea-Bissau; Liberia [14,628]; Nigeria; Sierra Leone [263,481,1180]; ?Togo; Zaire [233,896,991]

Cephalophus jentinki Thomas 1892

Jentink's Duiker

Côte d'Ivoire [936,1178]; Liberia [14,49,253,936]; Sierra Leone $[263,265,1180]$

Cephalophus monticola (Thunberg 1789)

II

(= Philantomba monticola)

Blue Duiker

Angola [518]; Benin [978]; Burundi [1179]; Cameroon; Central African Republic; Congo [691]; Côte d'Ivoire [768]; Equatorial Guinea: including Femando Po [182]; Ethiopia; Gabon [691]; ?Ghana; ?Guinea; Kenya; ?Liberia; Malawi [78,452]; Mozambique [1017,1020]; Nigeria [1]; Sierra Leone [263,481]; South Africa [1013,1020]; Sudan [503]; Swaziland; Tanzania [33]; ?Togo; Uganda [33,120]; Zaire [233,991]; Zambia [75]; Zimbabwe [1020]

Cephalophus nigrifrons rubidus Thomas 1901

Ruwenzori Black-fronted Duiker

Uganda

Cephalophus ogilbyi (Waterhouse 1838)

(except subspecies crusalbum)

Ogilby's Duiker

Cameroon; ?Côte d'lvoire [768,1178]; Equatorial Guinea: including Femando Po [108,182,1093]; Ghana; Liberia [14,49,628]; Nigeria [859]; Sierra Leone [481,1086,1180]; Togo

Cephalophus ogilbyi crusalbum Grubb 1978

White-legged Duiker

Gabon [430]

Cephalophus spadix Tne 1890

Abbott's Duiker

Tanzania

Cephalophus sylvicultor (Afzelius 1815)

Yellow-backed Duiker

Angola [518]; Benin [978]; ?Burkina Faso [944]; Cameroon; Central African Republic; Congo [691]; Côte d'Ivoire [768]; Equatorial Guinea [182]; Gabon [691]; ?Gambia; Ghana [560]; Guinea; Guinea-Bissau; Kenya; Liberia [14,628]; Nigeria [20]; Rwanda; Senegal [307]; Sierra Leone [263,481,1086, 1180]; Sudan [503]; Togo; Uganda [120]; Zaire [233,896]; Zambia [75]

Côte d'lvoire [768]; Liberia [49]; Sierra Leone [263,481,1180] 
Damaliscus dorcas (Pallas 1766)

(except subspecies dorcas)

Blesbok

South Africa [1014]

Damaliscus dorcas dorcas (Pallas 1766)

\section{Bontebok}

South Africa

Damaliscus hunteri (Sclater 1889)

Hunter's Hartebeest, Hirola

Kenya [33]; Somalia [337,367, 1007]; Tanzania [33]

Damaliscus lunatus (Burchell 1823)

(except subspecies korrigum)

Tsessebe

Angola [518]; Botswana [ 1020]; Burkina Faso [975]; Burundi [1128]; Ethiopia; Kenya [33]; Malawi (ex) [1066]; Mozambique [1017,1020]; Namibia [1020]; Rwanda; Somalia [337,367]; South .Africa [1013,1020]; Sudan [503]; Tanzania [33]; Uganda [33, I20]; Zaire; Zambia [75]; Zimbabwe [1020]

Damaliscus lunatus korrigum (Ogilby 1837)

III GH E 724

Korrigum, Tiang, Topi

Benin [978, I I3 I]; Burkina Faso [975]; Central African Republic; Chad; Mali [975]; Niger [872]; Nigeria (ex?) [19,859]; Senegal (ex) [307]; Sudan [503]

Dorcatragus megalotis (Menges 1894)

K $\quad 27,724$

Beira Antelope

?Djibouti; Ethiopia; Somalia $[337,367,1007]$

Gazella cuvieri (Ogilby 1841)

III TN E 27,724

Edmi Gazelle, Idmi, Cuvier's Gazelle

Algeria [1015]; Morocco [183]; Tunisia [1172]; Western Sahara [1113]

Gazella dama (Pallas 1766)

I $\quad E$

$27,724,855$

Dama Gazelle, Addra Gazelle

Algeria (ex?) [804]; ?Cameroon [449]; Chad [689,804]; ?Gambia; Libya [528,804]; Mali [804,975]; ?Mauritania; Morocco (ex?) [183]; Niger [4 19,804,805]; Nigeria [859]; Senegal (ex); Sudan [804,996]; Tunisia (ex); Western Sahara (ex?) [804, 1113]

Gazella dorcas (Linnaeus 1758)

III TN $\quad \mathrm{V} \quad 724$

(includes G. bennetti (Sykes 1831))

Dorcas Gazelle

Afghanistan; Algeria [804,1015]; Chad [689,804]; Djibouti; Egypt [347,835,954]; Ethiopia; India [38]; Iran; ?Iraq [464]; ?]srae] [347,464, I197]; ?Jordan [464]; Kuwait [464]; Libya [528]; Mali [804]; Mauritania [804]; Morocco [183,856]; Niger [804,805]; Nigeria (ex?) [610]; Pakistan [930]; Saudi Arabia [464]; Senegal [804]; Somalia [337,367]; Sudan [804]; Togo; Tunisia [928]; Western Sahara [804,1113]; Yemen [464]

Gazella gazella (Pallas 1766)

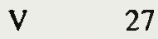

Mountain Gazelle

Egypt [355]; Israel [464]; Oman [464]; Saudi Arabia [464]; ?Syria [428]; Yemen [464]

Gazella leptoceros (F. Cuvier 1842)

III TN E

27

Rhim, Slender-horned Gazelle, Sand Gazelle

Algeria [485, 1015]; Chad [314,689]; Egypt [835,954]; Libya [528]; ?Mali [804]; ?Mauritania [804]; Niger [804]; ?Sudan [804]; Tunisia [990]; Western Sahara [804] 
Gazella rufifrons Gray 1846

Red-fronted Gazelle

?Benin [311]; Burkina Faso [311]; Cameroon [311]; Central African Republic [311]; Chad [311]; ?Ethiopia [309]; Gambia [311]; Ghana (ex?) [311]; Guinea-Bissau [311]; Mali [311]; Mauritania [311]; Niger [311]; Senegal [311]; Sudan [311]

Gazella soemmerringi (Cretzschmar 1826)

Soemmerring's Gazelle

Djibouti [309]; Ethiopia [309]; Kenya [309]; Somalia [309]; Sudan

Gazella spekei Blyth 1863

Speke's Gazelle

Ethiopia; Somalia $[337,367,1007]$

Gazella subgutturosa marica Thomas 1897

E $\quad 27,464$

(= G. leptoceros marica)

Arabian Goitred Gazelle

Bahrain [370]; Iraq [687]; Jordan; Kuwait; Oman; Saudi Arabia; United Arab Emirates; Yemen

Hemitragus hylocrius (Ogilby 1838)

V $27,38,99,100,262$

Nilgiri Tahr

India

Hemitragus jayakari Thomas 1894

E

27

Arabian Tahr

Oman [464]; United Arab Emirates: Abu Dhabi [279]

Hippotragus leucophaeus (Pallas 1766)

Ex $310,724,1014$

Bluebuck

South Africa (ex)

Hippotragus niger variani (Thomas 1916)

Giant Sable Antelope

Angola [724]

Kobus leche Gray 1850

II

V 27,724

Lechwe

Angola [518]; Botswana [1020]; Namibia [584]; [South Africa [1020]]; Zaire [991]; Zambia [75,525]

Nemorhaedus goral (Hardwicke 1825)

(except subspecies baileyi)

Goral

?Afghanistan; Bhutan; China [994]; India [204,381,660]; D.P.R. Korea [1184]; Korea Republic [1184]; Lao P.D.R. [271,417]; Nepal [756]; Pakistan [797,930]; Russia [106,239]; Thailand [96,151]

Nemorhaedus goral baileyi Pocock 1914

(includes N. cranbrooki)

Red Goral

China; India; Myanmar [955]

Oreotragus oreotragus porteousi Lydekker 1911 Western Klipspringer

Nigeria 
Oryx dammah (Cretzschmar 1826)

(=O.tao (H. Smith 1827))

$1 \quad$ E 27,724

Scimitar-horned Oryx

Algeria (ex) [804]; Burkina Faso (ex); Chad [804]; Egypt (ex?) [835]; ?Libya [528]; Mali [29]; Mauritania (ex) [804]; Morocco (ex) [183]; Niger [802,804,805]; Nigeria (ex) [503]; Senegal (ex) [804]; Sudan [804]; Tunisia (ex); Western Sahara (ex?) $[390,1113]$

$\begin{array}{llll}\text { Oryx leucoryx (Pallas 1777) } & \text { I } & \text { E } & 27,724\end{array}$

Arabian Oryx

Egypt (ex); Iraq (ex) [687]; 1srael (ex); Jordan (ex); [Oman]; Saudi Arabia (ex) [464]; United Arab Emirates; Yemen (ex)

Ovis ammon (Linnaeus 1758)

$11 \quad-\quad 239$

(except subspecies hodgsonii)

Argali

Afghanistan [440,793]; Armenia [106]; Azerbaijan [106]; China [985]; Georgia [106]; Kazakhstan [106]; Mongolia [695]; Pakistan [797,930]; Russia [106]; Tajikistan [106]; Turkmenia [106]; Uzbekistan [106]

Ovis ammon hodgsonii Blyth 1841

Tibetan Argali

239

Bhutan [386]; China; India [38,836]; Nepal [755,756]

Ovis canadensis Shaw 1804

Bighorn Sheep

Mexico [906]

(Non-CITES populations in Canada and USA)

$11 \quad-\quad 442,906$

Ovis musimon Pallas 1811

V $\quad 27,197$

(= O. ammon musimon, 0 . orientalis musimon)

Mediterranean Mouflon

[Austria]; [Belgium]; [Bulgaria]; [Czechoslovakia]; [Finland]; France: Corsica; [French Southem and Antarctic Territories: Kerguelen Islands]; [Germany]; [Hawaiian Islands]; [Hungary]; Italy: Sardinia; [Luxembourg]; [Netherlands]; [Poland]; [Romania]; [Spain]; [Switzerland];

Ovis oriensalis ophion Blyth 1841

$\begin{array}{lll}I & \text { V } & 27\end{array}$

$(=0$. aries ophion, $O$. ammon musimon (pro parte))

Cyprus Mouflon

Cyprus

Ovis vignei Blyth 1841

I $\quad-\quad 239$

( $=0$. ammon vignei, $O$. orientalis vignei)

Urial

Afghanistan; India [38,693,836]; Iran [646]; Oman; Pakistan [797,930]; Russia [1200], Turkmenia

Pantholops hodgsoni (Abel 1826)

Chiru, Tibetan Antelope

China [994]; India [38,836]

Redunca fulvorufula adamauae Pfeffer 1962

E

Western Mountain Reedbuck

Cameroon [311]; Nigeria [311]

Rupicapra rupicapra cartusiana Couturier 1938

E 665

Chartreuse Chamois

France 
RL Ref

Rupicapra rupicapra ornata Neumann 1899 Abruzzo Chamois

Italy: Abruzzo National Park

Rupicapra rupicapra tatrica Blahout 1972

Tatra Chamois

Czechoslovakia; Poland [396]

Tetracerus quadricornis (Blainville 1816)

Four-horned Antelope

India [38]; Nepal [756]

Tragelaphus buxtoni (Lydekker 1910)

Mountain Nyala

Ethiopia [309]

Tragelaphus oryx gigas (Heuglin 1863)

(= Taurotragus derbianus gigas)

Eastern Giant Eland

Cameroon [311]; Central African Republic [311]; Chad (ex?) [311]; ?Nigeria [311]; Sudan; Zaire

Tragelaphus oryx derbianus (Gray 1847)

(= Taurotragus derbianus derbianus, $T$. derbianus derbianus)

Western Giant Eland

Côte d'Ivoire; Gambia (ex); Guinea (ex?); Guinea-Bissau (ex?); Mali [975]; Senegal [39]

Tragelaphus spekei Speke 1863

$111 \mathrm{GH}$

E

27,724

Sitatunga, Marshbuck

Angola [518]; Benin [978]; Botswana [1020]; Burundi [1 128,1179]; Cameroon [557]; Central African Republic; Chad [689]; Congo [1031]; Côte d'lvoire; Equatorial Guinea [182]; Gabon [691]; Gambia; Guinea (ex); Guinea-Bissau (ex?); Kenya [33]; Namibia; Niger (ex); Nigeria [20,610]; Rwanda; Senegal [307]; Sudan [503]; Tanzania [33]; Togo; Uganda [33,120]; Zaire; Zambia [75]; ?Zimbabwe [1179] 


\section{REFERENCES}

1. Adeola, M.O. and Decker, E. 1987. Urilisation de la faune sauvage en milieu rural au Nigeria. Nature et Faune 3(4): 15-21.

2. Adler, H.J. 1990. The Crested Gibbon, Hylobates (Nomascus) concolor in the Nam Cat Tien Reserve, south Vietnam. Garmulax, Biology and Conservation in Vietnam 7: 6-10.

3. Ahmad, A. 1982. Preliminary investigations on food, behaviour and distribution of Muak Deer in northem areas. Palistan Joumal of Forestry 32(1): 24-27.

4. Al-Robaae, K. 1982. Northeast extension of the geographic distribution of Fennec Fox, Fennecus zerda Zimmerman, in the Arabian peninsula. Bullerin of the Basrah Natural History Museum 5: 61-64.

5. Alados, C.L. 1985. Distribution and status of the Spanish Ibex (Capra pyrenaica Schiaz). In: Lovari, S. (Ed.), The biology and management of mountain ungulates, Croom Helm, London, pp. 204-211.

6. Albignac, R. 1970. Notes éthologiques sur quelques Carnivores Malgaches: Le Cryptoprocia ferax. La Terre et lo Vie 24: $395-402$.

7. Albignac, R. 1970. Notes éthologiques sur quelques carnivores Malgaches: Le Fossa fossa (Schreber) Paris. La Terre et la Vie 24: 383-394.

8. Albignac, R. 1974. Observations éco-éthologiques sur le genre Eupleres, Viverride de Madagascar. La Terre el la Vie 28(3): 321-351.

9. Albignac, R. 1987. Priorités dans la conservation dus mammiferes non Primates de Madagascar. In: Anon, 1987. Priorités en matière de conservation des espèces d̀ Madagascar. IUCN, Gland.

10. Albrecht, H. 1976. Chimpanzees in Uganda. Oryx 13(4): $357-361$.

11. Alcobar, A.C. 1971. The last Almiquis (Solenodon cubanus) in captivity. Zoologische Garten N.F. Leipzig, 40: 1-3.

12. Aleem, A. 1979. Markhor population dynamics and food availability in Chitral Gol Wildlife Sanctuary. Pakistan Joumal of Forestry 29(3): 166-181.

13. Alkina, M.E. 1979. The mammals of Trinidad. Occassional Paper No.2. Deptmantment of Zoology, University of the West Indies, St. Augustine, Trinidad.

14. Allen, G.M. and Coolidge, H.J. 1930. Mammals of Liberia. In: Strong, R.P. The African Republic of Liberia and the Belgian Congo. Harvard University Press, Cambridge, Mass.

15. Allen, J.A. 19??. Notes on Solenodon paradoxus Brandt. Bulletin American Museum Natural History 24: 505-517.

16. Almeida, F.F. de 1988. Threatened species in Portugal. Species 11: 41-42.

17. Alvares, V.B. and Gonzales, A.C. 1991. The critical condition of hutias in Cuba, Oryx 25(4): 206-208.
18. Amstrup, S.C. and Wiig, O. 1991. Polar Bears. Proceedings of the Tenth Working Meeting of the IUCN/SSC Polar Bear Specialist Group, October 25-29, 1988, Sochi, USSR. IUCN, Gland.

19. Anadu, P.A. 1987. Progress in the conservation of Nigeria's wildlife. Biological Conservarion 41(4): 237-251.

20. Anadu, P.A. and Oates, J.F. 1982. The status of wildlife in Bendel State, Nigeria, with recommendations for its conservation. Report of Project 1613, to IUCN/WWF, Gland, Switzerland. Unpublished.

21. Anderson, E.M. 1987. A critical review and annotated bibliography of literature on the Bobcat. Colorado Division of Wildlife Terrestrial Wildlife Res., Species/Report No. 62.

22. Anderson, S., Woods, C.A., Morgan, G.S. and Oliver, W.L.R. 1983. Geocapromys brownii. Mammalian Species 201: $1-5$.

23. Anna, M. 1971. Report concemant le léopard d'Afrique dans la République du Tchad. Directorate of National Parks and Game Reserves in Tchad. Unpublished.

24. Anon. 1966. Provisional list of the fauna of Guyana. Joumal of the Guyana Museum and Zoo of the Royal Agricultural and Commercial Sociery 42: 58-60.

25. Anon. 1969. Threat to monkeys on Fernando Poo. Oryx 10(3): 146-147.

26. Anon. 1971. United Nations list of norionol parks and equivolent reserves. Hayez, Brussels.

27. Anon. 1972-1978. Red data book: Mammalia. IUCN, Morges.

28. Anon. 1975. Cuban Solenodon. Pp. 104-105. In: Walker, E.P. (Ed.), Mammals of the world. The John Hopkins University Press, Baltimore.

29. Anon. 1976. Consultants meeting on Addrx and oryx. IUCN, Morges, Switzerland, January 1976. Unpublished.

30. Anon. 1976. Solenodon paradoxus in southem Haiti. Joumal of Mammalogy 57(3): 591-592.

31. Anon. 1977. A new mammal record for Chins Hemitragus jemlohicus. Acto Zoologica Sinica 23(1): 116.

32. Anon. 1977. Protection, conservotion and management of threatened and endangered species in Egypt. Report of US Fish and Wildlife Service National Park Study Mission, July $30,1977$.

33. Anon. 1977. Report of the working group on the distribution and status of East African mammals, Phase 1: large mammals. East African Wildlife Society, Scientific and Technical Committee.

34. Anon. 1977. Report on the Status of Canadian Wildlife used by the Fur Indistry. Department of Industry, Trade and Commerce, Ottawa, Canada. 
35. Anon. 1978. Report of special consulting committee (Members: J.S. Gartan, C.J. Jones, A. Kortlandt, T.T. Struhsaker) convened by the Interagency Primate Steering Committee of the Nationsl Institutea of the Health, Washington, D.C., March 1978. Intemational Primate Protection League Newslenter 5(2): 14.

36. Anon. 1980. A world review of the Cetacea. Nature Conservancy Council, Peterborough.

37. Anon. 1981. Afghanistan. A Contribution ta a Conservation Strategy. Volume II: Appendixes. UNDP/FAO/National Parks and Wildlife Management Afghanistan, Rome 1981. FO:DP/AFG/78/007 Technical Report, Vol. II.

38. Anon. 1981. Rare and endangered animals of India. Government of India.

39. Anon. 1981. Senegal'a disappearing elephants. Oryx 16(2): 118-119.

40. Anon. 1982. New primate records for Rwanda. Oryx 16(4): 328 .

41. Anon. 1982. Relict population of Marsh Deer found. Oryx 16(4): 322.

42. Anon. 1982. Yaks. China Pictorial 9: 42-45

43. Anon. 1983. Inventaire des resources en faune sauvage el étude economique sur son utilisatian en zone rurales Haute-Volta. UNDP/FAO, Rome.

44. Anon. 1983. Kouprey sighted. Oryx 17(1): 45.

45. Anon. 1983. Virus threat to Lion-tailed Macaque. WWF Monthly Repon October 1983: 633-635.

46. Anon. 1984. Conservacionismo en Espana. Secretaria General técnica. (MOPU). Servicio de publicaciotes, pp. 40-42.

47. Anon. 1984. François Monkey in China. Onyx 18(1): 49.

48. Anon. 1984. Javan Rhino in Malaysia. Straits Times, 13 March.

49. Anon. 1984. Liberia's Sapo National Park. Oryx 18(1): 48.

50. Anon. 1984. Note on the status of the Woolly Spider Monkey. Laboratory Primate Newsletter 23(1): 4.

51. Anon. 1984. The Jaguars of Belize. The Ferret 5: 1-2.

52. Anon. 1984. Utah Prairie Dog reclassified to threatened. Endangered Species Technical Bulletin 9(6): 7-8.

53. Anon. 1984. Wildlife heritage. Bulletin of the Society for Conservation of Nature and Enviranment 14.

54. Anon. 1985. Cuademos Academia Nacional de Ciencias de Bolivia. Ciencias de la Naturaleza. Museo Nacional de Historia Natural, Zoologia, pp 3.

55. Anon. 1985. Determination of endangered staus and critical habitat for three beach mice; final rule. Report of US Fish and Wildlife Service, 58-85: 23872-23889.6 June 1985.

56. Anon. 1985. Listings made final for two mammals. Endangered Species Technical Bulletin 10(2): 1-3.

57. Anon. 1985. Transfer of Chinese population of Felis bengalensis bengalensis from Appendix 1 to Appendix II. Proposal submitted by the People's Republic of China to the 5 th meeting of the Conference of the Parties to CITES, Buenos Aires, Argentina.

58. Anon. 1985. Yet more Black-footed Ferrets. Oryx 19(1): 43.
59. Anon. 1986. New hope for the Southern Sea Otter. Endangered Species Technical Bulletin 11: 12-14.

60. Anon. 1987. Deteminatian of endangered status for the Mouns Graham Red Squirrel; final rule. Report of US Fish and Wildlife Service, 11:20994-20999.3 June 1987.

61. Anon. 1987. Proposed determination of endangered status for the Haulapai Vole; proposed rule. Repon of US Fish and Wildlife Service, 2-87: 306-309. 5 June 1987.

62. Anon. 1988. Cats in Djibouti. Cat News 9: 19.

63. Anon. 1988. Mamiferos de Venezuela. Lista y claves para su identificación. Universidad Central de Venezuela, Facultad de Agronomia/Museo del Instituto de Zoologia Agricola. Maracay, Venezuela.

64. Anon. 1988. The Asiatic Cheetsh lives on. Cat News 8: 11.

65. Anon. 1989. Rwanda: first sighting of rare monkey. Animal Kingdom 92(5): 21 .

66. Anon. 1990. Cheetah surviving in Iran. Cat News 13: 13.

67. Anon. 1990. Results of Tiger census in India. Cat News 13: 3 .

68. Anon. 1991. Aberdeen University Expedition to Madagascar 1989. Project No. 89/20/7. Flora and Fauna Preservation Society, London.

69. Anon. 1991. Biodiversity action plan for Indanesia. Draft August 1991 .

70. Anon. 1991. Black-footed Ferret recovery effort progresses towand reintroduction. Endangered Species Technical Bulletin 16(1): 1-5.

71. Anon. 1991. Golden Langur - distribution confusion. Oryx 25(3): 124.

72. Anon. 1991. Haulapai Mexican Vole (Microtus mexicanus hualpaiensis) recovery plan. Fish and Wildlife Service, Albuquerque, New Mexico.

73. Anon. 1992. Arabian Leopard survives in Saudi Arabia. Cat News 17: 16.

74. Anon. 1992. More Rusty-spotted Cats caught in South India. Cat News 17: 16

75. Ansell, W.F.H. 1978. The mammals of Zambia. National Parks and Wildlife Service, Chilanga.

76. Ansell, W.F.H. 1982. Some mammal species absent from or marginal to Malawi. Nyala 8(1): 23-29.

77. Ansell, W.F.H. 1982. The southeastern range limit of Manis tricuspis Rafinesque and the type of Manis tridentata Facillon. Mammalia 46(4): 559-561.

78. Ansell, W.F.H. and Dowsett, R.J. 1988. Mammals of Malawi - an annatated checklist and atlas. Trendrine, St. Ives, Comwall.

79. Aplin, O.V. 1894. Field-notes on the mammals of Uruguay. Proceedings of the Zoological Society of London 1894: 297-354.

80. Arita, H.T. and Humphrey S.R. 1988. Revision taxonomica de los murcielagos magueyeros del genero Leptonycteris (Chiroptera: Phyllostomidae).Acta Zoolagica Mexicana 29: 1-60.

81. Ashby, K.R. and Santiapillai, C. 1986. An assessment of the status of the Banteng (Bos javanicus) with particular reference to its interaction with the Water Buffalo (Bubalus bubalis). Tigerpaper 13: 10-20. 
82. Ashby, K.R. and Santiapillai, C. 1988. The status of the Banteng (Bos javanicus) in Java and Bali. Tigerpaper 15(4): 16-25.

83. Asibey, E.O.A. 1972. The present status of wildtife conservation in Ghana. In: Happold, D.C.D. (Ed.), Wildife conservation in West Africa. IUCN New Series 22, Morges.

84. Asibey, E.O.A. 1978. Primate conservation in Ghana. In: Chivers, D.J. and Lane-Petter, W. (Eda), Recens advances in primatology, val. 2, conservation. Academic Presa, London, pp. 55-74.

85. Augusto Tovar, S. 1971. Catalogo de mamiferos peruanos. Arales Cientificos, Lima, Peru 9: 18-37.

86. Aveling, C. 1984. Notes on the Golden Monkey, Cercopithecus miris kandti, of the Virunga volcanoes, Rwanda. African Joumal of Ecology 22(1): 63-64.

87. Aveling, C. and Aveling, R. 1989. Gorilla conservation in Zaire. Oryx 23(2): 64-70.

88. Aveling, C. and Harcourt, A.H. 1984. A census of the Virunga Gorillas. Oryx 18(1): 8-13.

89. Ayres, J.M. 1985. On a new species of squirrel monkey, genus Saimiri. Papeis Avulsos de Zoologia. Sao Paulo 36(14): 147-164.

90. Ayres, J.M. 1986. The conservation status of the White Uakari. Primate Conservarion 7: 22-26.

91. Ayres, J.M. and Johns, A.D. 1987. Conservation of White Uacaries in Amazonian várzea. Onyx $21:$ 74-80.

92. Ayres, J.M., Mittermeier, R.A. and Constable, I.D. 1982. Brazilian tamarins on the way to extinction? Oryx 16(4): 329-333.

93. Azzaroli, M.L. 1968. Second specimen of Mesaplodon pacificus the rarest living beaked whale. Monitore Zoologico Italiana (N.S.) 2(Suppl.): 67-69

94. Baal, F.L.J., Mittermeier, R.A. and Van Roosmalen, M.G.M. 1988. Primates and protected areas in Suriname. Oryx 22: 7-14.

95. Bagley, F. and Jacobs, J. 1985. Census technique for endangered Big-eared Bats proving successful. Endangered Species Technical Bulletin 10(3): 5-7.

96. Bain, J.R. and Humphrey, S.R. 1980. A profile of the endangered species of Thailand. Report No. 4. Office of Ecological Services, Florida State Museum.

97. Baird, R.W., Langelier, K.M. and Stacey, P.J. 1989. First reconds of False Killer Whales Pseudorca crassidens in Canada. Canadian Field Naturalist 103: 368-371.

98. Baker, A.N. 1977. Spectacled Porpoise, Phocoena dioptrica, new to the subantarctic Pacific Ocean. New Zealand Joumal of Marine and Freshwater Research I1(2): 401-406.

99. Balakrishnan, M. 1984. The larger mammals and their endangered habitats in the Silent Valley forests of south India. Biologicol Conservation 29(3): 277-286.

100. Balakrishnan, M. and Easa, P.S. 1986. Habitat preferences of the larger mammals in the Parambikulam Wildlife Sanctuary, Kerala, India. Biological Conservation 37: 191 200.

101. Balcomb, K.C. III and Nichols, G., Jr. 1982. Humpback Whale censuses in the West Indies. Repon of the Intemational Whaling Commission 32: 401-406.
102. Baldwin, L.A. and Teleki, G. 1972. Field research on baboons, drills and geladas: an historical, geographical and bibliographical listing. Primates 13: 427-432.

103. Balzano, E., Sweri, B. and Kouako, K. 1980. Répartition géographique de Papio anubis dans le nord-est et le centre-sud de Côte d'Ivoire. Primates 21(4): 524-537.

104. Bannikov, A.G. 1978. Asiatic Wild Ass in the Soviet Union and neighbouring countries. Unpublished.

105. Bannikov, A.G. 1978. The present status of the Bactrian Deer (Cervus elaphus bactrianus) in the USSR. In: Anon, Threatened deer. Proceedings of a working meeting of the Deer Specialist Group of the SSC. IUCN, Morges, pp. 159-172.

106. Bannikov, A.G. and Sokolov, V.I. 1984. Krasnaya Kniga SSSR. (2nd edition). Lesnaya Promiishlyennost, Moscow.

107. Barros Machado, A. de 1969. Mammiferos de Angola ainda nao citados ou pouco conhecides. Das Publicafaes Cullurais da Companhia de Diamantes de Angola 46: 93-232.

108. Basilio, A. 1962. La vića animal en la Guinea Espanola. Instituto de Estudios Africanos, Madrid.

109. Bastida, R. and Bastida, V.L. de 1984. Avistajes de cetaceos realizados por buques balleneros en aguas Argentinas. Revista del Museo Argentino de Ciencias Naturales 'Bemardino Rivadavia', Zoologia 13: 211-224.

110. Bastida, R. and Bastida, V.L. de 1984. Informe preliminac sobre los estudios de Ballena Franca Austral (Eubalaena australis) en la zona de la Peninsula Valdes (Chubut, Argentina). Revista del Museo Argentino de Ciencias Naturales 'Bemardina Rivadavia', Zoologia 13: 197-210.

111. Bauer, J.J. 1988. A preliminary assessment of the reintroduction success of the Asian One-homed Rhinoceros (Rhinoceros unicomis) in Bardia Wildlife Reserve, Nepal. Tigerpaper 15(4): 26-32.

112. Bauer, J.J. 1990. The analysis of plant-herbivore interactions between ungulates and vegetation on alpine grasslands in the Himalayan region of Nepal. Vegetation 90(1): 15-34.

113. Beddington, J.R. and Cooke, J.G. 1984. Assessments of the Northeast Atlantic stock of Minke Whales. Repurt of the Intemational Whaling Commission 34: 285-291.

114. Beddington, J.R., Cooke, J.G. and de la Mare, W.K. 1983. Estimations of the western North Pacific Sperm Whale population using the length distribution of the catches. Report of the Intemarional Whaling Commission 33: 747-749.

115. Beja, P.R. 1989. Coastal Oners in southwest Portugal. IUCN Otter Specialist Group Bulletin 4: 2-7.

116. Bejarano B., G. 1983. Status y distribución de las familias Cebidae y Callitrichidae en Bolivia. I Reunión Iberoamericana de Zoólogos de Vertebrados, La Rabida. 1977, pp. 685-701.

117. Bell, D.J., Hoth, J., Velazquez, A., Romero, F.J., Leon, L. and Aranda, M. 1985. A survey of the distribution of the Volcano Rabbit. Dodo 22: 42-48.

118. Beltran, J.F. 1990. Spanish Lynx Lynx prodina. Pp. 1112. In: Anon, Cat Specialist Group meeting reports, Cat News 12: 2-14. 
119. Bennen, E.L., Caldecott, J.O. and Davison, G.W.H. 1984. A wildlife survey of Ulu Temburong, Brunei. Umpublished.

120. Bere, R.M. 1962. The wild mammals of Uganda. Longmans, London.

121. Berkes, F., Anat, H., Kislalioglu, M. and Esenel, M. 1979. Distribution and ecology of Monachus monachus on Turkish coasts. In: Ronald, R. and Duguy, R. (Eds), The Mediterranean Monk Seal. UNEP Technical Series, Volume 1. Pergamon Press, Oxford, pp. 113-127.

122. Berta, A. 1982. Cerdocyon thous. Mammalian Species 186.

123. Berta, A. 1986. Aselocynus microtis. Mammalian Species 256.

124. Bertonatti, C. and Gonzalez, F. 1992. Lista de verebrados Argentinas en peligro de extinción. Fundacion Vida Silvestre Argentina. Boletin Tecrico No. 8.

125. Bertram, G.C.L. and Ricardo Bertrsm, C.K. 1973. The modem Sirenia: their distribution and status. Biologicol Joumal of the Linnean Society 5(4): 297-338.

126. Berzin, A.A. 1981. A note on the recent distribution and numbers of the White Whale in the Soviet far east. Repor of the Intemational Whaling Commission 31: 527-529.

127. Berzin, A.A. and Doroshenke, N.V. 1982. Distribution and sbundance of Right Whales in the North Pacific. Repon of the Intemational Whaling Commission 32: 381 383.

128. Berzin, A.A. and Vladimirov, V.L. 1983. (A new species of killer whale (Cetacea, Delphinidae) from Antarctic waters]. Zoologicheskii Zhumal 62(2): 287-295. (In Russian).

129. Best, G.A, Edmund-Blanc, F. and Witting, R.C. (Eds) 1962. Rowland Word's records of big game (Africo). (11th edition). Rowland Ward, London.

130. Best, P.B. and Shaughnessy, P.D. 1981. First record of the Melon-headed Whale Peponocephola electra from South Africa. Annals of the South African Museum 83(3): 33-47.

131. Bevanger, K. 1992. Report on the Norwegian wolverine (Gulo gulo L.). Small Camivore Conservation 6: 8-10.

132. Bhat, H.R. Lion-tailed Macaque in Kamstake. WWF Indio Newsletter 4(1): 9.

133. Bhatti, M.U. and Pilleri, G. 1982. Status of the Indus Dolphin population (Plolanisto indi Blyth, 1859) between Sukkur and Guddu barrages in 1979-1980. Investigations on Cetaceo 13: 245-252.

134. Bibikov, D.1., Filimonov, A.N. and Kudaktin, A.N. 1983. The status and management of the Wolf population in the USSR. Acta Zoologica Fennica 174: 269-271.

135. Bismark, M. 1981. Preliminary survey of the Proboscis Monkey at Tanjung Puting Reserve, Kalimantan Tigerpaper 8(1): 26 .

136. Biswas, B., Ghose, R.K. and Ghosal, D.K. 1986. The lesser cats in eastern India. WWF Monthly Report June 1986: $143-147$

137. Bjārvall, A. 1983. Scandinavia's response to a natural repopulation of Wolves. Acla Zoolagica Fennica 174: 273-275.

138. Bjärvall, A. and Ullstrōm, S. 1986. The mammals of Britain and Europe. Croom Helm, London.
139. Blsb, J. and Nowak, E. 1978. Rote liste der sãugetien (Mammalia). ln: Blab, J., Nowak, E. and Trautmann, W. (Eds), Rote liste der gefahrdeten tiere und pflanzen in der Bundesrepublic Deutschland. Kilda Verlag, Germany, pp. 13-14.

140. Bleisch, W. and Chen Non 1990. Conservation of the Black-crested Gibbon in China. Onyx 24: 147-156.

141. Blom, A., Alors, M.P.T, Feistner, A.T.C., Bames, R.F.L. and Barnes, K.L. 1992. Primates of Gabon current ststus and distribution. Oryx 26: 223-234.

142. Blomquist, L. 1980. Distribution and status of the Snow Leopard (Panthero uncia). Tigerpaper 7(4): 15-20.

143. Blood, B.R. 1990. Taxonomy and distribution of Sigmodon in Californis, USA. Bulletin of the South Califomia Academy of Sciences 89(2): 86-93.

144. Bloucb, R.A. and Atmosoedirdjo, S. 1978. Preliminary report on the status of the Bawesn Deer (Axis kuhli). In: Anon, Threatened deer. Proceedings of a working meeting of the Deer Specialist Group of the SSC. IUCN, Morges, pp. 49-55.

145. Boeadi. and Bergmans, W. 1987. First record of Dobsonic minor (Dobson, (1879)) from Sulawesi, Indonesis (Mammalia: Megachiroptera). Bulletin, Zoologisch Museum, Universiteit van Amsterdam 11: 69-74.

146. Bohor, B.S. et al. 1988. First record of Dinomys branickii for Venezuela. Joumal of Mammalogy 69: 433.

147. Bojovic, D.R. 1983. The state and position of Wolves (Canis lupus L.) in Yugoslavia. XV Congr. Int. Fauna Cinegética y Silvestre. Trujillo 1981, pp. 581-584.

148. Bolton, B.L. and Lat, P.K. 1978. The westem Hare-wallaby, Lagorchestes hirsutus (Gould) (Macropodidae) in the Tanami Desert. Austrolian Wildlife Research 5: 285-293.

149. Bolton, M. 1978. Notes on the current status and distribution of some large mammals in Ethiopis. Mammalia 37(4): 562-586

150. Bonner, W.N. 1989. The natural history of seals. Christopher Helm, London.

151. Boonsong Lekagul and McNeely, J.A. 1977. Mammals of Thailand. Association for the Conservation of Wildlife, Bangkok.

152. Booth, A.H. 1956. Some Gold Coast mammals not included in Cansdale's "provisional check list". Journal of the West African Science Association 2: 137-138.

153. Booth, A.H. 1956. The distribution of primates in the Gold Coast. Joumal of the West African Science Associotion 2: 122-133.

154. Booth, A.H. 1957. Observations on the natural history of the Olive Colobus Monkey Procolobus verus van Beneden. Proceedings of the Zoological Sociery of London 129: $421-430$.

155. Booth, A.H. 1958. The zoogeography of West African primates: a review. Bulletin de l'Institut Fondamental de l'Afrique Noire 20: 587-622.

156. Booth, A.H. 1960. Small mammals of West Africa. Longmans, London.

157. Borner, M. 1976. Sumatra's Orang-utans. Oryx 13(3): 290-293.

158. Borner, M. 1977. Leopards in western Turkey. Oryx 14(1): 26-30. 
159. Borner, M. and Frey, R. 1975. Progress report: Orangutan rehabilitation, Bohorok. WWF Project 733: Gunung Leuser Reserve, Sumatra.

160. Botev, B. and Peshev, T., (Eds) 1985. The red data book of the People's Republic of Bulgaria. Vol. 2. Animals. Publishing House of the Bulgarian Academy of Sciences, Sophia.

161. Bothma, J. du P. 1975. Conservation status of the larger mammsls of southem Africa. Biological Conservation 7: 87-95.

162. Bourlière, F., Minner, E. and Vuattoux, R. 1974. Les grands mammiferes de la région de Lamto, Côte d'lvoire. Mammalia 38(3): 433-447.

163. Brack-Egg, A. 1978. Situacion actual de las nutrias (Lutrinae, Mustelidae) en el Peru. In: Duplaix, N. (Ed.), Otzers. Proceedings of the first working meeting of the IUCN/SSC Oter Specialist Group, Paramaribo, Suriname 27-29 March 1977. IUCN, Morges, pp. 76-84.

164. Breitenmoser, U. 1990. Lynx in Switzerland. P. 11. In: Anon, Cat Specialist Group meeting reports. Cat News 12: 2-14.

165. Brennan. E.J. 1985. De Brazza's Monkeys (Cercopithecus neglectus) in Kenya: census, distribution, and conservation. American Joumal of Primasology 8(4): 269-277.

166. Brett, R.A. 1990. The Black Rhino sanctuaries of Kenya. Pachyderm 13: 31-34.

167. Brooks, D. 1991 . Some notes on terrestrial mustelids in the central Paraguayan Chaco. Mustelid ami Viverrid Conservation 4: 5-6.

168. Brotoisworo, E. 1978. Nature conservation in Indonesia and its problems, with special reference to primates. La: Chivers, D.J. and Lane-Petter, W. (Eds), Recent advances in primatalogy, vol. 2 : conservation, pp. 31-40.

169. Brown, L.H. 1975. A report on the wildlife situation in Ethiopia, November 1975. Repon to World Wildlife Fund.

170. Brown, P. 1979. Mammals observed in Brunei. Brunei Museum Joumal 4(3): 174-179.

171. Brownell, R.L. 1975. Phocoena dioptrica. Mammalian Species (American Society of Mammalogists) 66: 1-3.

172. Brownell, R.L. 1983. Phocoena sinus. Mammalian Species (Americat Society of Mammalogists) 198: 1-3.

173. Brownell, R.L. Ir and Praderi, R. 1984. Phocoena spinipinnis. Mammalian Species (American Society of Mammalogists) 217: 1-4.

174. Bruner, P.L. and Pratt, H.D. 1979. Notes on the status and natural history of Micronesian bats. Elepaio 40(1): 1-4.

175. Budnitz, N. and Dainis, K. 1975. Lemur catta: ecology and behavior. Pp. 219-235. In: Tattersall, 1. and Sussman, R.W. (Eds), Lemur Biology, Plenum Press.

176. Buijs, D. and Dudok van Heel, W.H. 1979. Bodyplan of a male Sperm Whale (Physeter macrocephalus) stranded near Breskens, Netherlands. Aquatic Mammals 7(1): 27-32.

177. Burmeister, H. 1861. Reise durch die La Plata-Staaten. Halle 2: $387-432$.

178. Burt, W.H. and Stinton, R.A. 1961. The mammals of El Salvador. Miscellaneous Publications of the Musuem of Zoology, University of Michigan 117: 1-69.

179. Burton, J.A. and Pearson, B. 1987. Collins guide to the rare mammals of the world. Collins, London.
180. Butynski, T.M. and Koster, S.H. 1989. The status and conservation of forests and primates on Bioko Island (Femando Po), Equatorial Guinec. Impenetrable Forest Conservation Project, World Wildlife Fund, Kampala, Uganda.

181. Būtzler, W. 1980. Présence et répartition des gorilles, Gorilla gorilla gorilla (Savage and Wyman, 1847) au Came roun. Saugetierkundliche Mineilungen 28(I): 67-79.

182. Cabrera, A. 1929. Catalogo descriptivo de los mamiferos de la Guinea Espanola. Memorias de lo Sociedad Espagnol de Historia Natural, Madrid 16: 1-121.

183. Cabrera, A. 1932. Los mamiferos de Marnecos. Trabajos del Museo Nacional de Ciencias Nalurales, Serie Zoologica 57: 1-361.

184. Cabrera, A. 1957. Catalogo de los mamiferos de America del sur, vol. 1, Metatheria, Unguiculata, Carnivora. Revista del Museo Argentino de Ciencias Naturales 'Bemardino Rivadavia', Ciencias Zoologicas 4(1): 1-307.

185. Cabrera, A. 1960. Catalogo de los mamiferos de America del sur, vol. II., Sire.ia, Perissodactyla, Artiodactyla, Lagomorpha, Rodentia, Cetacea. Revista del Museo Argennino de Ciencias Naturales 'Bemardino Rivadovia', Ciencias Zoologicas 4(2): 309-732.

186. Cai Gui-quan and Feng Zuo-jian 1981. On the occurrence of the Himalayan Musk Deer (Moschus chrysogaster) in China and an approach to the systematics of the genus Moschus. Acta Zootaxonomica Sinica 6: 106-110.

187. Caldwell, D.K. 1971. Cuvier's Beaked Whale Ziphius covirostris from Barbados. Bulletin Southem Califomia Academy of Science 70: 52-53.

188. Campbell, H.W. and Gicca, D.F. 1978. Reseña preliminar del estado actual y distribucion del manati (Trichechus manatus) en México. Ann. Inst. Biol. Univ. Autonama Mérico. 49(1): 257-264.

189. Cansdale, G.S. 1964. The Volta dam may help wildlife in Ghana. Onyx 7(4): 168-171.

190. Čaputa, A., Hoľ̌ik, J. and Berger, Z. 1982. Atlas chránených zivočichov Slovenska. Obzor, Bratislava.

191. Carbyn, L.N. (Ed.) 1983. Wolves in Canada and Alaska: their status, biology and management. Canadian Wildlife Service Report 45.

192. Carroll, J.B. 1981. The wild status and behaviour of the Rodrigues Fruit Bat Pteropus rodricensis. A repon of the 1981 field study. Dodo 18: 20-29.

193. Carroll, J.B. 1985. The flying foxes (Pleropus spp.) of the western Indian Ocean islands. Bat News 4: 4-5.

194. Carroll, R.W. 1986. Status of the Lowland Gorilla and other wildlife in the Dzanga-Sangha region of southwestem Central African Republic. Primote Conservation 7: 38-41.

195. Carroll, R.W. 1988. Elephants of the Dzanga-Sangha dense forest of south-western Central African republic. Pachyderm 10: 12-15.

196. Carvatho, C.T. de 1957. Alguns mamiferos do Acre ocidental. Boletin do Museu Paraense Emilio Goeldi, $n$. ser. Zoologia 6: 1-22.

197. Cassols, F. 1985. Management and conservation of the Sardinian Mouflon (Ovis musimon Schreber). An outline. In: Lovari, S. (Ed.), The biology and management of mountain ungulates, Croom Helm, London, pp. 197-203. 
198. Cassola, F. (Ed.) 1986. La Lontra in ltalia. World Wildlife Fund Serie Atti e Studi No. 5, Rome.

199. Castello, H.P. 1984. Registros del elefante marino, Mirounga leonina (Camivora, Phocidae) en las costas del Atlantico S.O., fuera del area de cria. Revista del Museo Argenrino de Ciencias Naturales 'Bemardino Rivadavia', Zaalagia 13: 235-243.

200. Castello, H.P. and Pinedo, M.C. 1977. Os visitantes ocasionais de nosso litoral. Natureza Revista 3: 40-46.

201. Castilla, J.C. 1982. Nuevas observaciones sobre conducta, ecologia y deosidad de Lutra felina (Molina 1782) (Camivora: Mustelidae) en Chile. Publicaciones Ocasionales del Museo Nacional de Historia Natural, Santiago 38: 197-206.

202. Castro, N. 1976. Guidelines for the conservation of primates in Peru. In: Anon, First Inter-American conference on conservation and utilizarion of American nonhuman primates in biomedical research. Scientific Publication no. 317. Pan American Health Organization, Washington, D.C., pp. 216-234.

203. Catten, P.E. and Glade, A.A. 1989. Management of the Vicuña Vicugna vicugna in Chile: use of matrix model to assess harvest rates. Biological Conservanion 49(2): 131 140.

204. Cavallini, P. 1990. Status of the Goral (Nemorhaedus goral) in Himachal Pradesh, India. Caprinae Specialist Group News 5: 46.

205. Ceballos, G. 1989. Diversity and conservation status of mustelids in Mexico. Mustelid and Viverrid Conservarion $1: 8$.

206. Ceballos, G. and Wilson, D.E. 1985. Cynomys mexicanus. Mammalian Species (American Society of Mammalogists) 248: pp 1-3.

207. Chakraborty, S. 1976. On a collection of mammals from Bhutan. Records of the Zoological Survey of India 68: 1-20.

208. Chakraborty, S. 1978. The Rusty-spotted Cat, Felis rubiginosa, 1. Geoffroy, in Jammu and Kashmir. Joumal of the Bombay Natural History Sociery 75(2): 478-479.

209. Chambers, M.R. and Bani, E. 1988. Custom, tradition and the Dugong (Dugong dugon) in Vanuatu. Naika December 1988: 17-25.

210. Chapman, D.G. 1983. Some considerations on the status of stocks of southern hemisphere Minke Whales. Report of the Intemarional Whaling Commission 33: 311-314.

211. Chapman, J.A. aod Feldhamer, G.A. 1982. Wild mammals of North America. Biology, management and economics. The John Hopkins University Press, Baltimore.

212. Chapman, J.A. and Flux, E.C. 1990. Rabbits, Hares and Pikas. Status survey and conservation action plan. IUCN, Gland.

213. Chapman, P.J. and Chapman, L.L. 1982. Otter survey of Ireland 1980-81. The Vincent Wildlife Trust, London.

214. Charles-Dominique, P. 1974. Ecology and feeding behaviour of five sympatric lorisids in Gabon. In: Martin, R.D., Doyle, G.A. and Walker, A.C. (Eds), Prosimian Biology, University of Pittsburgh Press, Pittsburgh, pp. 131-150.
215. Charles-Dominique, P. 1974. Vie sociale de Peradicticus potto (Primates, Lorisides): Etude de terrain en forêt equatoriale de l'Ouest Africain au Gabon. Mammalia $38(3)$ : $358-380$.

216. Chasen, F.N. 1935. On mammals from Siam. Joumal Siam Sociery, Natural History Supplement 10: 31-57.

217. Chasen, F.N. 1940. A handlist of Malaysian mammals. Bulletin of the Raffles Museum 15: 1-208.

218. Chehébar, C.E., Gallur, A., Giannico, G., Gottelli, M.D. and Yorio, P. 1986. A survey of the Southem River Otter Lutra provacax in Lanin, Puelo and Los Alerces National Parks, Argentina, and evaluation of its conservation status. Biological Conservation 38: 293-304.

219. Child, G. and Savory, C.R. 1964. The distribution of large mammal species in southern Rhodesia. Amoldia 1(14): 1-15.

220. Chivers, D.J. 1977. The lesser apes. In: Rainier, Prince and Bourne, G.H. (Eds), Primate conservation, Academic Press, New York, pp. 539-598.

221. Chom, J. and Hoffmann, R.S. 1978. Ailuropoda melanaleuca. Mammalian Species (American Society of Mammalogists) 110: 1-106.

222. Choudhury, A. 1987. Notes on the distribution and conservation of Phayre's Leaf Monkey and Hoolock Gibbon in India. Tigerpaper 14: 2-6.

223. Choudhury, A. 1993. The Clouded Leopard in Assam. Oryx 27: 51-53.

224. Choudhury, A. 1991. Ecology of the Hoolock Gibbon (Hylobates hoolock), a lesser ape in the tropical forests of north-eastem India. Joumal of Tropical Ecology 7: 147153.

225. Choudhury, A.U. 1985. Distribution of Indian One-homed Rhinoceros. Tigerpaper 12(2): 25-30.

226. Choudhury, D.K.L. 1986 Elephants in north-east India. WWF Monthly Report January 1986: 7-17.

227. Christensen, P.G.S. 1980. The biology of Bettongia penicillata Gray 1837 and Macropus eugenii (Desmarest 1817 ) in relation to fire. Forest Department of Western Australia Bulletin No. 91

228. Chundawat, R.S. 1990. Lynx survey in Nubra Valley, Ladakh. Wildlife Insritute of India Newsletter 5(2): 42-44.

229. Clark, B. 1983. African Wild Ass. Oryx 17(1): 28-31.

230. Clark, B. 1983. Israel restores Asiatic Wild Ass. Oryx 17(3): 113 .

231. Clark, T.W. 1991. Restoration of the endangered Blackfooted Ferret. Mustelid and Viverrid Conservation 4: 9-10.

232. Cohen, J.A. 1978. Cuon alpinus. Mammalien Species (American Society of Mammalogists) 100: 1-3.

233. Colyn, M., Dudu, A. and Mbaelele, M.M. 1987. Exploitation du petil et moyen gibier des forêts ombrophiles du Zaire. 1. Consommation qualitative dans le milieu rural. 2. Analyse de la commercialisation du giber à Kisangani (Haut-Zaire). Nature et Faune 3(4): 22 39.

234. Condy, P.R. 1978. The distribution and abundance of Southern Elephant Seals Mirounga leanina (Linn.) on the Prince Edward lslands. South African Joumal of Antarctic Research 8: 42-48. 
235. Condy, P.R. 1984. The population of the Southem Elephant Seal, Mirounga leonina, at Marion Island, 1973-1983. Sowth African Journal of Science 80(1): 26-27.

236. Conry, P.J. 1989. Gaur Bos gaunus and development in Malaysia. Biological Conservation 49: 47-65.

237. Corbet, G.B. 1969. The taxonomic status of the Pygmy Hippopotamus Choeropsis liberiensis from the Niger delta. Joumal of Zoology, London 158: 387-394.

238. Corbel, G.B. 1971. Provisional distribution maps of British mammals. Mammal Review 1(4/5): 95-144.

239. Corbet, G.B. 1978. The mammals of the Palaearctic region: a zaxonomic review. British Museum (Natural History), Comell University Press, London.

240. Corbet, G.B. and Hanks, J. 1968. A revision of the elephant shrews, Family Macroscelididae. Bulletin of the British Museum (Natural History) 16(2): 1-111.

241. Corbet, G.B. and Harris, S. 1991. The hondbook of British mammals. (3rd edition). Blackwell Scientific Publications, Oxford.

242. Corbet, G.B. and Southem, H.N. 1977. The handbook of British mammals. (2nd edition). Blackwell Scientific Publications, Oxford.

243. Corbet, G.B. and Hill, J.E. 1991. A world list of mammalian species. (3rd edition). Natural History Museum Publications. Oxford University Press, Oxford.

244. Corbet, G.B. and Hill, J.E. 1992. Mammals of the Indo Malayan region. Oxford University Press, Oxford.

245. Corbet, G.B. and Yalden, D.W. 1972. Recent reconds of mammals (other than bats) from Ethiopia. Bulletin of the British Museum (Natural History) Zoology 22(8): 211-252.

246. Correa-Vians, M. 1986. [Distribution and status of the West Indian Manatee in Venezuela]. Serv. Nac. Fauna Silvestre (Venezuelo). (In Spanish).

247. Cowan, I.M. and Holloway, C.W. 1978. Geographical Jocation and current conservation status of the threatened deer of the world. In: Anon, Threalened deer. Proceedings of a working meeting of the Deer Specialist Group of the SSC. JUCN, Morges, pp. 11-22.

248. Crespo, J.A. 1974. Comentarios sobre nuevas localidades para mamiferos de Argentina y de Bolivia. Revista del Museo Argentino de Ciencias Naturales Bemardino Rivadavia", Ciencias Zoologicas 11: 1-31.

249. Criel, D. 1989. The situation of the Otter in Flanders, Belgium. IUCN Otter Specialist Group Bulletin 4: 37-38.

250. Crockett, C. and Wilson, W.L. 1980. Survey of Sumatran primates: diversity and abundance in a shrinking paradise. Tigerpaper 7(2): 1-5.

251. Cunha Vieira, C. da 1955. Lista remissiva dos mamiferos do Brasil. Arquivos de Zoologia do Estado de Saa Paulo 8(2): $341-474$.

252. Currier, M.J.P. 1983. Felis concolor. Mammalian Species (American Society of Mammalogists) 200: 1-7.

253. Curry-Lindahl, K. 1974. Jenlink's Duiker (Cephalophus jentink) in Liberia. Mammalia 38: 187.

254. Daan, S. 1980. Long term changes in bat populations in the Netherlands. Luiro 1-3: 95-118.

255. Dalquest, W.W. 1968. Additional notes on mammals from Mozambique. Joumal of Mammalogy 49: 117-121.
256. Daly, R.H. 1990. Arabian Leopard Panthero pardus nimr. P. 4. In: Anon, Cat Specialist Group meeting report. Cat News 12: 2-14.

257. Dandelot, P. 1965. Distribution des quelques espèces de Cercopithecidae en relation avec lea zones de végétation de l'Afrique. Zoologie Africaine 1(1): 167-176.

258. Daniel, M.J. 1970. Bat sightings on Kapiti 1sland, New Zealand, 1906-1969. Proceedings of the New Zealand Ecological Society, 17: 136-138.

259. Dao, V.T. 1977. Sur quelques rares mammiferes au nord du Vietnam. Mineilungen aus dem Zoologischen Museum in Berlin 53: 325-330.

260. Dào van Tiên 1967. Sur les formes de l'Ecureuil bicolore, Rotufa bicolor (Rodentia, Sciuridae) au Vietnam et les regions voisines. Mitteilung Zoologischen Musuem in Berlin 43: 215-224.

261. Dào van Tiên 1978. Sur une collection de mammiferès du plateau de Moc Chan. Mitteilung Zoologischen Musuem in Berlin 54: 377-391.

262. Davidar, E.R.C. 1978. Distribution and status of the Nilgiri Tahr (Hemirragus hylocrius) - 1975-78. Journal of the Bombay Natural History Society 75(3): 815-844

263. Davies, A.G. 1987. The Gola Forest Reserves, Sierra Leone, wildlife conservation and forest management. IUCN, Gland.

264. Davies, G. 1990. The Gola project in Sierra Leone: report on the viverrids. Mustelid and Vivernid Conservation 2: 4 5 .

265. Davies, G. and Birkenhager, B. 1990. Jentink's Duiker in Sierra Leone: evidence from the Freetown Peninsula. Oryx 24: $143-146$.

266. Davies, G. and Payne, J. 1982. A faunal survey of Sabah. IUCN/WWF Project No. 1692. WWF Malaysia.

267. de Beaufort, F. 1991. Mammols of Europe. Stalus, repartition, conservotion. Museum National d'Histoire Naturelle, Paris.

268. Deag, J.M. 1977. The status of the Barbary Macaque (Macaca sylvanus L.) in captivity and factors influencing its distribution in the wild. In: Rainier, Prince and Boume, G.H. (Eds), Primate conservation. Academic Press, New York, pp. 267-287.

269. Dechau, C.P. 1990. Rainforest yields 'extinct' lemur. New Scientist 27 Jenuary.

270. Defler, T.R. 1983. A remote park in Colombia. Oryx 17(1): 15-17.

271. Delacour, J. 1940. Liste provisoire de mammiferes de 1'Indochine française. Mammalia 4: 20-29.

272. Delacour, J. 1951. La systématique des gibbons Indochinois. Mammalia 15: 118-123.

273. Delany, M.J. 1975. The rodents of Uganda. British Museum (Nałural History), London.

274. Delibes, M. 1983. Distribution and ecology of the Iberian carnivores: a shon review. XV Congr. Int. Founa Cinegética y Silvestre. Trujillo 1981 pp. 359-378.

275. Department of the Environment, Iran. 1978. Persian Faliow Deer (Dama mesopolamica). Status repon. In: Anon, Threatened deer. Proceedings of a working meeting of the Deer Specialist Group of the SSC. IUCN, Morges, pp. 23-25. 
276. Depierre, D. and Gillet, H. 1974. Le mouflon en Ennedi (Tchad). Bois et Forêts des Tropiques 158.

277. Deuve, J. 1961. Note sur la famille Tapiridae au Laos. Bulletin de la Societé Royale des Sciences Narurelles de Laos 1: 47-48.

278. Devaraj, B.V. 1983. Distribution study of the Nicobar Crab-esting Macaque. IUCN/SSC Primate Specialist Group Newsletuer 3: 27-28.

279. Dickinson, P., Furley, C.W. and Greenwood, A.G. 1983. Arabian Tahr rediscovered in United Arab Emirates. Oryx 17(2): 93-94.

280. Diersing, V.E. 1980. Systematics of flying squirrels, Glaucomys volans (Linnaeus) from Mexico, Guatemala and Honduras. Southwestem Naturalist 25: 157-172.

281. Diersing, V.E. 1981. Systematic status of Sylvilagus brasiliensis and $S$. insonus from North America. Joumal of Mammalogy 62: 539-556.

282. Dieterlen, F. and Heim de Balsac, H. 1979. Ecology and taxonomy of the shrew Soricidae of the Kivu area Zaire, Central Africa. Saeugetierkundliche Minteilungen 27(4): 241-287.

283. Dietz, J.M. 1985. Chrysocyon brachyunus. Mammalian Species 234.

284. Dinerstein, E., Mehta, J.N. 1989. The Clouded Leopard in Nepal. Oryx 23: 199-201.

285. Dippenaar, N.J. 1980. New species of Crocidura from Ethiopia and northem Tanzania. Annals of the Transvaal Museum 32: 125-154.

286. Dippenaar, N.J. and Meester, J.A.J. 1989. Revision of the luna - fumosa complex of Afrotropical Crocidura. Annals of the Transvaal Museum 35: 1-47.

287. Dirschl, H.J., Mbugua, S.W. and Wetmore, S.P. 1978. Preliminary results for an aerial census of livestock and wildlife in Kenya's rangelands. Kenya Rangeland Ecological Monitoring Unit. Ministry of Tourism and Wildlife, Kenya. Aerial Survey Technical Repon Series 3.

288. Dirschl, H.J. and Wetmore, S.P. 1978. Grévy's Zebra. Abundance and distribution in Kenya 1977. Kenya Rangeland Ecological Monitoring Unit, Nairobi. Aerial Survey Technical Report Series 4.

289. Dobias, R.J. 1987. Elephants in Thailand: an overview of their status and conservation. Tigerpaper 14: 19-24.

290. Domning, D.P. 1982. Commercial exploitation of manatees Trichechus in Brazil c. 1785-1973. Biological Conservarion 22(2): 101-126.

291. Donadio, A. 1978. Some comments on otter trade and legislation in Colombia. In: Duplaix, N. (Ed.), Oners. Proceedings of the first working meeting of the IUCN/SSC Oter Specialist Group, Paramaribo, Suriname 27-29 March 1977. IUCN, Morges, pp. 34-42.

292. Dorji, D.P. and Santiapillai, C. 1989. The status, distribution and conservation of the Tiger Panthera tigris in Bhutan. Biological Conservarion 48: 311-319.

293. Dorst, J. and Dandelot, P. 1970. A field guide to the larger mammals of Africa. Collins, London.

294. Douglas-Hamilton, 1. 1987. African Elephant population atudy. Pachyderm 8: 1-10.

295. Douglas-Hamilton, 1. 1987. African Elephants: population trends and their causes. Oryx 21:11-24.
296. Douglas-Hamilton, I., Froment, J.M. and Doungoubé, G. 1985. Aerial censua of wildlife in the north of the Central African Republic. Report to CNPAF/ WWF/ IUCN/ PNUD/ FAO. Unpublished.

297. Dowset, R.J. and Dowsen-Lemaire, F. 1991. Flore et faure du bassin du Kouilou (Congo) et leur exploitation. Tauraco Rescarch Reporn No. 4. Tauraco Press, Belgique.

298. Dragesco, A. 1983. Le Massif de Termit en Niger: un sanctuaire à protéger. Unpublished.

299. Duguy, R. 1986. [Record of Walrus (Odobenus rosmanus) on the coast of Gironde, France]. Mammalia 50(4): 563564. (In French).

300. Dunbar, R.I.M. and Dunbar, E.P. 1974. Ecology of population dynamics of Colobus guereza in Ethiopia. Folia Primatologica 21 : 188-208.

301. Dunbar, R.I.M. and Dunbar, E.P. 1976. Contrasts in social siructure among Black-and-white Colobus Monkey groups. Animal Behaviour 24: 84-92.

302. Dunn, A. 1991. A study of the relative abundance of primate and duiker populations in Liberia. Project No. 3216, WWF/ FDA Wildlife Survey.

303. Dupuy, A. 1971. SOS pour la conservation de la nature en Mauretanie. Science et Nature 108: 31-34.

304. Dupuy, A.R. 1971. Les oiseaux et les mammiferes de la cuvette du Djoudj (Delta du fleuve Sénégal). Bulletin de I'I.F.A.N. 33: 237-248.

305. Dupuy, A.R. 1971. Statut actuel des primates au Sénégal. Bulletin de l'Insritut Fondamental de l'Afrique Noire 33(1-2): 467-478.

306. Dupuy, A.R. 1973. Premier inventaire des mammiferes du Parc National de Basse Casamance (Sénégal). Bulletin de l'Insritut Fondamental de l'Afrique Noire 35: 186-197.

307. Dupuy, A.R. and Verschuren, J. 1977. Wildlife and parks in Senegal. Oryx 14(1): 36-46.

308. Duran, J.C., Cattan, P.E. and Yanez, J.L. 1985. The Grey Fox Canis griseus (Gray) in Chilean Patagonia (southem Chile). Biological Conservation 34(2): 141-148.

309. East, R. 1988. Antelopes, global survey and regional acrion plans. Pan 1. East and Norheast Africa. IUCN/SSC Antelope Specialist Group, Gland.

310. East, R. 1989. Antelopes, global survey and regional action plans. Pan 2. Southem and South-Central Africa. IUCN/SSC Antelope Specialist Group, Gland.

311. East, R. 1990. Antelopes, global survey and regional action plans. Parn 3. West and Central Africa. IUCN/SSC Antelope Specialist Group, Gland.

312. Eaton, R.L. 1976. The Brown Hyena: A review of biology, status and conservation. Mammalia 40(3): $377-400$.

313. Economic Commission for Europe. 1991. European red list of globally threatened animals and plants. United Nations, Washington, D.C.

314. Edmond-Blanc, F., Rothschild, A. de and Rothschild, E. de 1962. Contribution à l'étude des grandes ongules dans le nord du Bourkou (Tchad). Mammalia 26: 489-493.

315. Egler, S.G. 1983. Current status of the Pied Bare-face Tamarin in Brazilian Amazonia. IUCN/SSC Primate Specialist Group Newsletter 3: 20. 
316. Eisenberg, J.F. 1989. Mammals of the Neotropics. Vol.1. Panama, Colombia, Venezuela, Guyana, Suriname, French Guiana. Univeraity Chicago Prese, Chicago.

317. Eisenberg, J.F. 1990. South Americ an cats and the Florida Panther Felis concolor coryi.

318. Eisenberg, J.F. and McKay, G.M. 1970. An annotated checklist of the recent mammals of Ceylon with keys to the species. Ceylon Joumal of Science 8(2): 69-99.

319. Eisenberg, J.F., O'Connell, M.A. and August, P.V. 1979. Density, productivity and distribution of mammals in two Venezuelan habitats. In: Eisenberg, J.F. (Ed.), Veriebrate ecology in the northem Neotropics. Smithsonian, Washington, D.C.

320. Eisentraut, M. 1973. Die wirbeltierfauna von Femando Poo und West Kamerun. Bonner Zoologische Monographien 3: 1-428.

321. Eisentraut, M. 1986, Über dans vorkommen des ChacoPekati, Casagonus wagneri, in Bolivien. Bonner Zoologische Beiträge 37: 43-47.

322. Elbl, A., Rahm, U.H. and Mathys, G. 1966. Les mammiferes et leurs tiques dans la Forêt du Ruggege (République Rwandaise). Acla Tropica, Parasitologie 23: 223-263.

323. Ellermaft, J.R. and Morrisson-Scott, T.C.S. 1951. Checklist of Palaearctic and Indian mammals 1758-1946. British Museum (Natural History), London.

324. Ellerman, J.R. and Morrisson-Scott, T.C.S. 1966. Checklist of Palaearctic and Indian mammals. (2nd edition). British Museum (Natural History), London.

325. Elliot, K.M. 1983. The Otter (Lutra tutra) in Spain. Mammal Review 13(1): 25-34.

326. Else, J.G. 1983. Can we save the De Brazza's Monkey in Kenya? Komba 1983(2-3): 27-28.

327. Emlen, J.T. and Schaller, G.B. 1960. Distribution and status of the Mountsin Gorilla Gorilla gorillo beringei 1959). Zoologica 45: 41-52.

328. Erlinge, S. 1978. The status of the Oter in Sweden. In: Duplaix, N. (Ed.), Oners. Proceedings of the first working meeting of the IUCN/SSC Oner Specialist Group, Paramaribo, Suriname 27-29 March 1977. IUCN, Morges, pp. 144-146.

329. Estes, J.A. 1980. Enhydris lutris. Mammalian Species (American Society of Mammalogists) 133: 1-8.

330. Estrada, A.R. and Ferrer, L.T. 1987. Distribucion del manati antillano, Trichechus manotus (Mammalia:Sirenia), en Cuba. 1. Region occidental. Poeyana 354.

331. Ewer, R.F. 1973. The camivores. Weidenfeld and Nicolson, London.

332. Fa, J.E. 1982. A survey of population and habitat of the Barbary Macaque Macaca sylvanus $L$. in north Morocco. Biological Conservation 24(1): 45-66.

333. Fa, J.E. 1983. The Barbary Macaque - the future. Oryx 17(2): 62-67.

334. Fa, J.E. 1986. An important new locality for the Barbary Macaque (Mocaca sylvanus) in Morocco. Primate Conservation 7: 31-34.

335. Fa, J.E. 1986. On the ecological status of the Barbary Macaque Macaca sylvanus $L$. in north Morocco: habitat influences veraus human impact. Biological Conservation $35: 215-258$.
336. Fa, J.G. 1989. The genus Macaca: a review of taxonomy and evolution. Mammal Review 19: 45-81.

337. Fagotto, F. 1985. Larger animals of Somalia in 198.4. Environmental Conservation 12: 260-264.

338. Fairley, J.S. 1975. An Irish beast book. Blackstaff Press, Belfast.

339. Fay, J.M. and Agnagna, M. 1991. Forest Elephans population in the Central African Republic and Congo. Pachyderm 14: 3-19.

340. Fayard, A., Saint Girons, M.C. and Maurin, H. (Eds) 1984. Atlas des mammiferes sauvages de France. Ministère de L'Environment, Société Française pour l'Etude et la Protection des Mammiferes, Paris.

341. Fedigan, L.M. 1986. Demographic trends in the Alouatta palliata and Cebus capucinus populations of Santa Rosa National Park, Costa Rica. In: Else, J.G. and Lee, P.C. (Eds), Primale ecology and conservation, volume 2. Cambridge University Press, Cambridge.

342. Feiler, A. 1988. Die säugetiere der inseln im Golf von Guinea Zoologische Abhandlungen staatliche Museum fir Tierkunde Dresden 44: 83-88.

343. Feldhamer, G.A. 1980. Cervus nippan. Mammalian Species (American Society of Mammalogists) 128: 1-7.

344. Felton, H. 1958. Weitere säugetiere aus El Salvador. Senckenbergiena Bialogica 39: 213-326.

345. Feng Zuo-jian, Zheng Chang-lin and Cai Gui-quan 1980. [On mammals from south-eastern Xizang (Tibet)]. Acta Zoologica Sinica 26(1): $91-97$. (In Chinese).

346. Ferguson, W.W. 1981. The systematic position of Canis aureus lupaster and the occurrence of Canis lupus in North Africa, Egypt and Sinai. Mammalia 45: 459-465.

347. Ferguson, W.W. 1981. The systematic position of Gazello dorcas (Artiodactyla: Bovidae) in lstael and Sinai. Mammalia 45: 453-457.

348. Festetics, A. (Ed.) 1980. Der Luchs in Europa. Verbreitung, wiedereinbïrgenung, räuber-beu'e-beziehung. Kilda Verlag.

349. Field, P.A.G. 1974. Report on a feasibility study on a proposed conservation area in southern Darfur, Sudan. Unpublished.

350. Fini, F. and Macchia, G. 1977. /l Gran Paradisa. N. Zanichelli, Bologna.

351. Flannery, T.F. 1987. An historic record of the New Zealand Greater Shonttailed Bat, Mystacina robusto (Microchiroptera: Mystacinidae) from the South Island, New Zealand. Australian Mammal. 10(1): 45-46.

352. Flannery, T.F. 1990. The mammals of New Guinea. Rober Brown and Associates, Carina, Queensland.

353. Fleagle, J.G. and Mittermeier, R.A. 1980. Locomotor behaviour, body size and comparative ecology of seven Surinam monkeys. American Joumal of Physical Anthropology 52(3): 301-314.

354. Flores Villela, O. and Gerez, P. 1988. Conservacion en Mexico: sintesis sobre venebrados terrestres, vegetacion y uso del suelo. Instituto Nacional de Investigaciones Sobre Recuros Bioticos, Xalapa.

355. Flower, S.S. 1932. Notes on the recent mammals of Egypt, with a list of the species recorded from that kingdom. Proceedings of the Zoalogical Saciery of London: 369-450. 
356. Flynn, R.W. and Abdullah, M.T. 1984. Distribution and status of the Sumatran Rhinoceros in Peninsular Malaysia. Biological Conservation 28: 253-273.

357. Fonseca, F.P. 1983. Protection of the Wolf in Portugal. XV Congr. Int. Fauna Cinegética y Silvestre. Trujillo 1981 pp. 661-664.

358. Fonseca, G.A.B. da 1985. Observations on the ecology of the Muriqui (Brachyteles arachnoides E. Geoffroy, 1806): implications for its conservation. Primate Conservation 5: 48-52.

359. Fooden, J. 1975. Taxonomy and evolution of Liontail and Pigtail Macaques (Primates: Cercopithecidae). Fieldiana Zoology 67: 1-169.

360. Fooden, J. 1976. Primates obtained in peninsular Thailand June-July, 1973, with notes on the distribution of continental southeast Asian leaf-monkeys (Presbytis). Primates 17: 95-118.

361. Fooden, J. 1982. Taxonomy and evolution of the sinica group of macaques: 3 . Species and subspecies accounts of Macaca assamensis. Fieldiana Zoology (N.S.) 10: 1-52.

362. Fooden, J. 1983. Taxonomy and evolution of the sinica group of macaques: 4. Species account of Macaca thibetana. Fieldiana Zoology (N.S.) 17: 1-20.

363. Fooden, J., Quan Guoqiang, Wang Zongren and Wang Yingxiang 1985. The stumptail macaques of China. American Joumal of Primatolagy 8(1): 11-30.

364. Fordyce, R.E., Mattia, R.H. and Wilson, G.J. 1979. Stranding of a Cuvier's Beaked Whale Ziphius cavirastris Cuvier, 1823, at New Brighton. New Zealand. Mauri Ora 7: 73-82.

365. Frade, F. 1985. Aves e mamíferos das ithas de Sāo Tomé e do Principe, notas de sistemática e de protecçāo à Fauna. Conferéncia Intemacional das Africanistas Ocidentis, Comunicaçoes-Zoologia e Biologia Animal 4(6th session): 137-150.

366. Fuller, T.K., Johnson, W.G. Franklin, W.L. and Johnson, K.A. 1987. Notes on the Patagonian Hog-nosed Skunk (Conepaus humboldti) in southem Chile. Joumal of Mammalogy 68(4): 864-867.

367. Funaioli, U. and Simonetta, A.M. 1966. The mammalian fauna of the Somali Republic: status and conservation problems. Monitore Zoologico Italiano 74(Suppl.): 285-295.

368. Gakahu, C.G. 1991. African thinoceroses: challenges continue in the 1990s. Pachyderm 14:42-45.

369. Gallagher, M.D. 1975. The dugong Dugong dugon (Sirenia) at Bahrain, Persian (Arabian) Gulf. Joumal of the Bombay Natural History Society 73: 211-212.

370. Gallagher, M.D. and Harrison, D.L. 1975. The terrestrial mammals of Bahrain. Joumal of Bambay Natural History Saciery 72: 407-421

371. Ganzhom, J.U. and Rabesoa, J. 1986. Sightings of Ayeayes in the eastem rainforest of Madagascar. Primate Conservation 7: 45 .

372. Gardner, A.L. 1971. Notes on the Little Spotted Cat Felis rigrina oncilla in Costa Rica. Jaumal of Mammalogy 52: 464-465.
373. Gartlan, J.S. 1973. Influences of phylogeny and ecology on variations in the group organization of primates. In: Menzel, E.W. (Ed.), Precultural primate behaviour. Symposium of the 4th Intemational Congress of Primatology, vol. 1. Karger, Basel, pp. 88-101.

374. Gartan, J.S. 1975. The African coastal rain forest and its primates: threatened resources. In: Bermant, G. and Lindburg, D.G. (Eds), Primate utilization and conservation. Wiley and Sons, New York, pp. 67-82.

375. Gartlan, J.S. 1980. Conservation status of western Gorillas. Unpublished.

376. Gartlan, J.S. and Brain, C.K. 1968. Ecology and social variability in Cercopithecus aethiops and C. mitis. In: Jay, P.C. (Ed.), Primates: studies in adaptation and variability. Holt, Rinehart and Winston, New York, pp. 253-293.

377. Gartian, J.S. and Struhsaker, T.T. 1972. Polyspecific associations and niche separation of rain-forest anthropoids in Cameroon, West Africa. Joumal of Zoolagy, London 168: $221-266$

378. Garzon, P., Palacios, F. and Garzon, J. 1980. Situacion actual del oso pardo (Ursus arctos pyrenaicus Fischer, 1890) en Espana y datos sobre su alimentacion en la Cordillera Cantábrica. I Reunión lberoamericana de Zoólogos de Vertebrados, La Rábida 1977, pp. 681-683.

379. Gaskin, D.E. 1972. Whales, dolphins and seals. Heinemann, Auckland.

380. Gaskin, D.E., Amold, P.W. and Blair, B.A. 1974. Phocoena phocoena. Mammalian Species (American Society of Mammalogists) 42: 1-8.

381. Gaston, A.J., Garson, P.J. and Hunter, M.L. Jr. 1983. The status and conservation of forest wildlife in Himacha! Pradesh, westem Himalayas. Bialogical Conservation 27(4): 291-314.

382. Gatinot, B.L. 1974. Précisions sur la répartition du colobe bai (Colobus badius) et de la mone de Campbell (Cercopithecus mona). Mammalia 38(4): 71 1-716.

383. Gee, E.P. 1952. Possible occurrence of the Snub-nosed monkey (Rhinopithecus roxellanae) in Assam. Joumal of the Bombay Natural History Society 51: 264-265.

384. Gee, E.P. 1967. A note on the occurrence of the Malayan Sun Bear Helarctos malayanus (Raffles) within Indian limits. Joumal of the Bombay Natural History Sociery 64: 352-354.

385. Gee, E.P. 1967. Occurrence of the Brown Bear Ursus arctos Linn., in Bhutan. Joumal of the Bombay Natural History Society 64: 551-552.

386. Gee, E.P. 1967. Occurrence of the Nayan or Great Tibetan Sheep, Ovis ammon hadgsoni Blyth, in Bhutan. Joumal of the Bombay Natural History Saciety 64: 553554.

387. Gee. E.P. 1967. Occurrence of the Snow Leopard, Panthera uncia (Schreber), in Bhutan. Joumal of the Bombay Natural History Saciety 64: 552-553.

388. George, G.G. 1979. The status of endangered Papua New Guinea mammals. In: Tyler, M.J. (Ed.), The status of endangered Australasian wildlife. Royal Zoological Society of South Australia, pp. 94-100.

389. Ghiglier, M.D. 1984. Realm of the Mountain Gorillas. Swara 7(2):24-27.

390. Gillet, H. 1965. L'oryx algazelle et l'addax du Tchad. La Terre et la Vie 112(3): 257-272. 
391. Gillet, H. 1971. L'oryx algazelle et l'addax. Distribution géographie. Chance de survie. Compte Rendu Sommaire des Séances de la Société de Biogéographie 405: 177-189.

392. Ginsberg, J.R. and Macdonald, D.W. 1990. Foxes, wolves, jackals and dogs, an action plan for the conservation of canids. IUCN, Gland.

393. Gittins, S.P. and Akonda, A.W. 1982. What survives in Bangladesh? Tigerpaper 9(4): 5-11.

394. Glade, A.A. 1988. Red list of Chilean terrestrial verrebrates. Chilean Forest Service, Santiago.

395. Glander, K.E. 1983. The effects of trapping and removal on tamarin populations in Pers. IUCN/SSC Primate Specialist Group Newsletter 3: 19-20.

396. Glowacinski, Z. 1992. Polish red data book of animals. Panstwowe Wydawnictwo Rolnicze i Lesne, Warsaw.

397. Godfrey, L. and Vuillaume-Randriamanantena, M. 1986. Hapalemur simus: endangered lemur once widespread. Primate Conservation 7: 92-96.

398. Gogoi, P.C. 1981. A sanctuary for the Golden Langur. Tigerpaper 8(2): 7-9.

399. Goldman, C.A. and Taylor, M.E. 1990. Liberiictis kuhni. Mammalian Species (American Society of Mammalogists) 348.

400. Gonzalez, J.C. 1989. Guia para la identificacion de los murciélagos del Uruguay. Serie de Divulgacion No. 2. Museu da Maso Antonio Larranaga.

401. Gonzalez, J.C. and Vellejo, S. 1980. Niotas sobra Vampyraps linearus (Geoffroy) del Uruguay. Comunicaciones Zoologicas del Musea de Historia Natural, Montevideo 10(144): 1-8.

402. González, G.C. and Leal, C.G. 1984. Mamíferos Silvestres de la Cuenca de Mérico. Programme on Man and the Biosphere (MAB, UNESCO)/Instituto de Ecología y Museo de Historia Natural de la Ciudad de México. Editorial LIMUSA, México.

403. Goodall, R.N.P. and Galeazzi, A.R. 1985. A review of the food habits of the small cetaceans of the Antarctic and Sub-antanctic. In: Siegfried, W.R., Condy, P.R. and Laws, R.M. (Eds), Antarctic nutrient cycles and food webs. Springer-Verlag, Berlin, pp. 566-572.

404. Goodwin, G.G. 1934. Mammals collected by A.W. Anthony in Guatemala, 1924-1928. Bulletin of the American Museum af Natural History 68(1): 1-60.

405. Gondon, G., Riney, T., Toop, J., Lawrie, B.C. and Godwin, M.D. 1985. Observations on the Queensland Hairy-nosed Wombat Lasiorhinus kreffiii (Owen). Bialogical Conservation 33:165-195.

406. Gosàlbez, J. (Ed.) 1987. Histària natural dels Paisos Catalans. 13 amfibis, rèprtils $i$ mamífers. Enciclopédia Catalana S.A., Barcelona.

407. Grachev, Y.A. and Smimova, E.D. 1982. (Ecology of the European Brown Bear (Ursus arctos isabellinus) in the Aksu-Jabagly State Reserve (west Tian-Shan).] Zoalogicheskii Zhumal 61(8): 1242-1252. (in Russian).

408. Gray, G.G. and Simpson, C.D. 1980. Ammotragus lervia. Mammalian Species (American Society of Mammalogists) 144: 1-7.

409. Green, J. and Green, R. 1981. The Otter (Lutra lutra) in westem France. Mammal Review 11: 181-187.
410. Gray, J.E. 1861. List of Mammalia, tortoises and crocodiles collected by M. Mouhot in Camboja. Proceedings of the Zoological Socieny of London 1861: 135-140.

411. Green, M.J.B. 1978. Himalayan Musk Deer (Moschus maschiferus moschifenus). In: Anon, Threatened deer. Proceedings of a working meeting of the Deer Specialist Group of the SSC. IUCN, Morges, pp. 56-64.

412. Green, M.J.B. 1979. Tahr in a Nepal National Park. Oryx 15(2): 140-144.

413. Greet, M.J.B. 1982. Status, distribution and conservation of the Snow Leopard in north India. Intemational Pedigree Book of Snow Leopards 3: 6-10.

414. Green, M.J.B. 1985. Aspects of the ecology of the Himalayan Musk Deer. Ph.D. Thesis, Universily of Cambridge, Cambridge.

415. Green, M.J.B. 1986. The distribution, status and conservation of the Himalayan Musk Deer Moschus chrysogaster. Biological Conservation 35: 347-375

416. Greig, J.C. 1983. The South Aftican Southern Right Whale census, 1983. African Wildlife 37(5): 184.

417. Gressitt, J.L. 1970. Biogeography of Laos. Pacific Insects Monograph 24: 573-626.

418. Grettenherger, J. 1984. W National Park in Niger - a case for urgent assistance. Oryx 18(4): 230-236.

419. Grettenberger, J.F. and Newby, J.E. 1986. The status and ecology of the Dama Gazelle in the Air and Ténéré National Nature Reserve, Niger. Biological Conservation 38: 207-216.

420. Grimwood, 1.R. 1969. Notes on the distribution and status of some Penuian mammals. Special publication No. 21, American Committee for International Wildlife Protection and New York Zoological Society.

421. Groombridge, B. 1984. A new locality for the Lion-tailed Macaque. Oryx 18(3): 144-147.

422. Groves, C.P. 1971. Systematics of the genu's Nycticebus. In: Anon, Proceedings of the Third Intermational Congress of Primatology, vol. 1. S. Karger, Basel, pp. 44-53.

423. Groves, C.P. 1972. Systematics and phylogeny of gibbons. In: Rumbaugh, D.M. (Ed.). Gibbons and Siamang, vol. 1, Karger, Basle, pp. 1-89.

424. Groves, C.P. 1974. Horses, asses and zebras in the wild . David and Charles, London.

425. Groves, C.P. 1975. The taxonomy of Moschus (Mammalia, Artiodactyla), with particular reference to the Indian Region. Joumal of the Bombay Natural History Society 72(3): 662-676.

426. Groves, C.P. 1980. Speciation in Macaca: the view from Sulawesi. In: Lindburg, D.G. (Ed.), The Macaques: sudies in ecology, behaviour and evalution. Van Nostrand and Reinhold Co., New York.

427. Groves, C.P., Andrews, P. and Home, J.F.M. 1974. Tans River Colobus and Mangabey. Oryx 12(5): 565-575.

428. Groves, C.P. and Lay, D.M. 1985. A new species of the genus Gazella (Mammalia: Artiodactyla: Bovidae) from the Arabian Peninsula. Mammalia 49(1): 27-36.

429. Grubb, P. 1973. Distrihution, divergence and speciation of the Drill and Mandrill. Folia Primatologica 20: 161-177. 
430. Grubb, P. 1978. A new antelope from Gabon. Zoological Joumal of the Linnaean Society 62(4): 373-380.

431. Grubb, P. 1978. The Potto (Perodicticus potto: Primates, Lorisidae) in Nigeria and adjacent territories. Bulletin de l'Institut Fondamental de l'Afrique Noire 40(4): 900-913.

432. Grubb, P. 1982. The systematics of Sino-Himalayan musk deer (Moschus), with particular reference to the species described by B.H. Hodgson. Saugetierkundliche Mineilungen 30: 127-135.

433. Grubh, B.R. and Pereira, M.J. 1965. Standings of Finner Whale (Balaenoptera physalus (Linn.)) near Virar (Thana District) aod at Bombay, Maharashtra State. Joumal of the Bombay Natural History Society 62: 550-551.

434. Grzimek, B. 1978. Kameruns Urwald ist die Heimat der Gorillas. Das Tier 12: 16-19, 40-41.

435. Guggisberg, C.A.W. 1975. Mammals of eastern Africa. Sapra Studio, Nairobi

436. Guggisberg, C.A.W. 1975. Wild cats of the world. David and Charles, London.

437. Gunaratna, R. 1986. Whales off Sri Lanka. Sri Lanka Wildife $2: 5-7$.

438. Gunderson, V. 1977. Some observations on the ecology of Colobus badius temmincki, Abuko Nature Reserve, the Gambia, West Africa. Primates 18(2): 305-314.

439. Gupta, R.D. 1986. Elephants in northern India. WWF Monthly Report January 1986: 1-6.

440. Habibi, K. 1977. The mammals of Afghanistan, their distriburion and status. Ministry of Agriculture, Kabul.

441. Haimoff, E.H., Yang Xiao-Jun, He Swing-Jing and Chen Nan 1987. Conservation of gibbons in Yunnan Province, China. Oryx 21: 168-173.

442. Hall, E.R. 1981. The mammals of North America. 2 vols. (2nd edition). Wiley, New York.

443. Haltenorth, T. and Diller, H. 1977. Mammals of Africa. (English translation), Collins, London.

444. Hamilton, P.H. 1981. The Leopard Panthera pardus and the Cheetah Acinonyx jubatus in Kenya: ecology, status, conservation, management. Report prepared for the Office of Endangered Species. U.S. Fish and Wildlife Service, Washington, D.C.

445. Hamilion, W.J., Jr. and Whitaker, J.O., Jr. 1979 Mammals of the Eastem United States. (2nd edition). Comstock Publishing Associates a division of Cornell University Press, Ithaca, USA.

446. Han, C. 1982. [The status of the research on the Chinese River Dolphin (Lipotes vexillifer) in China.] Acta Theriologica Sinica 2(2): 245-252. (In Chinese).

447. Hancock, J. 1984. Fea's Muntjac. Oryx 18(2): 115.

448. Handley, C.O. 1976. Mammals of the Smithsonian Venezuelan project. Brigham Young University Science Bulletin, Biology Series 20(5): 1-89.

449. Happold, D.C.D. 1973. Large mammols of West Africa. Longmans, London.

450. Happold, D.C.D. 1973. The Red-crowned Mangabey, Cercocebus torqualus in western Nigeria. Folio Primatologica 20: 423-428.

451. Happold, D.C.D. 1986. The Mammals of Nigeria Clarendon Press, Oxford
452. Happold, D.C.D. and Happold, M. 1989. The mammsls of Zambia. Muala 14(1): 5-20.

453. Haque, M.N. and Siddiqi, N.A. 1988. Status of Slow Loris (Nycticebus coucang) in Bangladesh with some information on its food preferences. Tigerpaper 15(2): 1821 .

454. Harcourt, A.H. 1981. Can Uganda's Gorillas survive? A survey of the Bwindi Forest Reserve. Biological Conservation 19(4): 269-282.

455. Harcourt, A.H, Fossey, D. and Sabater-Pi, J. 1981. Demography of Gorillo gorilla. Joumal of Zoology. Landon 195: 215-233.

456. Harcour, A.H., Stewart, K.J. and Inahoro, I.M. 1989. Gorilla question in Nigeria. Oryx 23: 7-13

457. Harcour, A.H. and Fossey, D. 1981. The Virunga Gorillas: decline of an 'island' population. African Joumal of Ecology 19: 83-97.

458. Harding, R.S.O. 1983. A survey of endangered primates in Sierra Leone. IUCN/SSC Primate Specialist Group Newsletter 3: 22.

459. Harding, R.S.O. 1984. Primates of the Kilimi area, Sierra Leone. IUCN/SSC Primate Specialist Group Newsletter 4: 32-34.

460. Harrington, F.H. and Paquet, P.C. 1982. Wolves of the world. Noyes Publications, New Jersey.

461. Harrington, J.E. 1975. Field observations of social behavior of Lemur fulvus fulvus. Pp. 259-279. In: Tattersall. I. and Sussman, R.W. (Eds), Lemur Biology, Plenum Press.

462. Harris, C.J. 1968. Otters. Weidenfeld and Nicolson, London.

463. Harrison, D.L. 1964. The mammals of Arabia, Vol. I, Insectivora, Chiroptera and Primates. Benn, London.

464. Harrison, D.L. 1968. The mammals of Arabia, Vol. II, Camivora, Artiodactyla and Hyracoidea. Benn, London.

465. Harrison, D.L. 1972. The mammals of Arabia. Vol. III. Lagomorpha, Rodentia. Benn, London.

466. Harrison, D.L. and Bates, B.J.J. 1991. The Mammals of Arabia. (2nd edition). Harrison Zoological Museum Publication, Sevenoaks.

467. Harrison, D.L. and Jennings, M.C. 1980. Occurrence of the Noctule, Myctalus noculo Schreber, 1774 (Chiroptera: Vespertilionidae) in Oman, Arabia. Mammalia 44(3): 409 410.

468. Harrison, J. 1964. An introduction to the mammals of Sabah. The Sabah Society, Kota Kinabalu.

469. Harrison, J. 1974. An introduction to the mammals of Singapore and Malaysia. (2nd impression). Singapore Branch, Malayan Nature Society.

470. Harrison, M. 1988. A new species of guenon (genus Cercopithecus) from Gahon. Joumal of Zoology, London 215(3): $561-575$

471. Harrison. M.J.S. 1988. A new species of guenon (genus Cercopithecius) from Gabon. Joumal of Zoology, London 215(3): $561-575$

472. Harrison, T. 1970. The Orang-utan situation, 1970. Biological Conservarion 3(1): $45-46$. 
473. Hart, J.A. and Thomas, S. 1986. The Ituri Forest of Zaire: primate diversily and prospects for conservation. Primale Conservation 7: 42-44.

474. Hartman, D.S. 1974. Distribution, status and conservation of the manatee in the United States. US Fish and Wildlife Service National Fish Wildlife Lab. Contract Report. 14 16: 1-246.

475. Harvey, M.J., Cassidy, J.J. and O'Hagan, G.G. 1979. Status of endangered bats, Myotis sodalis, M. grisescens and Plecotus townsendii ingens in Arkansas. Proceedings Arkansas Academy of Science 33: 81.

476. Hassinger, J.D. 1973. A survey of the mammals of Afghanistan resulting from the 1965 Street expedition. Fieldiana Zoology 60: 1-195.

477. Han, R.T. 1934. A manatee collected by the American Museum Congo expedition, with observations on the recent manatees. Bulletin of the American Museum of Natural History 66: 533-566.

478. Hanon, J., Smar, N. and Thomson, K. 1984. In urgent need of protection - habitat for the Woolly Spider Monkey. Oryx 18(1): 24-29.

479. Hayman, R.W. 1961. The red goral of the north-east frontier region. Proceedings of the Zoological Sociery of London 136: 317-324.

480. Hayman, R.W. 1963. Mammals from Angola, mainly from the Lunda district. Das Publicą̧oes Culturais da Companhia de Diamantes de Angola 66: 81-139.

481. Haywood, A.H.W. 1932. Sierra Leone, the preservation of wildife. Joumal of the Society for the Preservation of the Fauna of the Empire (N.S.) 19: 21-33.

482. Haywood, A.H.W. 1932. The Gambia: the preservation of wildlife. Joumal of the Society for the Preservation of the Fauna of the Empire (N.S.) 19: 34-37.

483. Heaney, L.R., Gonzales, P.C. and Alcala, A.C. 1987. An annotated checklist of the taxonomic and conservation status of land mammals in the Philippines. Silliman Joumal $34(1-4): 32-66$

484. Heggberget, T.M. and Myberget, S. 1979. Den norske bestand au oter 1971 - 1977. Fauna 32: 89-95.

485. Heim de Balsac, H. 1936. Biogéographie des mammiferes et des oiseaux de l'A frique du Nord. Bullerin Biologique de France et de Belgique 21(Suppl.): 1-447.

486. Heltne, P.G., Freese, C. and Whitesides, G. 1976. A field survey of nonhuman primates in Bolivia. Pan American Health Organization, Washington, D.C.

487. Hemmer, H. 1978. Nachweis der Sandkatze (Felis margarita harrisoni Hemmer, Grubb and Groves, 1976) in Jordanien. Zeitschrift für Säugetierkunde 43(1): 62-64.

488. Hensley, A.P. and Wilkins, K.T. 1988. Leptonycteris nivalis. Mammalian Species (American Society of Mammalogists) 307.

489. Heptner, V.G., Nasimovich, A.A. and Bannikov, A.G. 1988. Mammals of the Soviet Union. Volume I. Artiodactyla and Perissodactyla. Smithsonian Institution Libraries and The National Science Foundation, Washington, D.C.

490. Hemandez-Camacho, J. and Cooper, R.W. 1976. The nonhuman primates of Colombia. In: Thorington, R.W., Jr and Heltne, P.G. (Eds), Neotropical primates: field studies and conservation. National Academy of Sciences, Washington, D.C., pp. 35-69.
491. Herrenschmidt, V. 1990. Lynx Lynx lynx in France and Switzerland. P. 11. In: Anon, Cat Specialist Group Meeting Reports. Cat News 12: 2-14.

492. Hershkovit,, P. 1954. Mammals of Northem Colombia preliminary repon No. 7: Tapirs (Genus Tapines), with a systematic review of American species. Proceedings of the United States Narional Museum 103(3329): 465-496.

493. Hershkovilz, P. 1977. Living New World monkeys (Platymhini), vol. 1. University of Chicago Press, Chicago.

494. Hershkovit., P. 1979. The species of sakis, Genus Pithecia (Cebidae, Primates) with notes on sexual dichromatism. Folia Primatologica 31: 1-22.

495. Hershkoviz, P. 1982. Neotropical deer (Cervidae) Pan 1. Pudus, Genus Pudu Gray. Fieldiana Zoolagy, New Series 11: 1-86.

496. Hershkovitz, P. 1987. The taxonomy of South American sakis, genus Pithecia. American Joumal of Primatology 12: $387-468$.

497. Hershkovil., P. 1988. Origin, speciation and distribution of South American titi monkeys, genus Callicebus. Proceedings of the Academy of Natural Sciences, Philadelphia 140: 240-272.

498. Hill, D.S. and Phillipps, K. 1981. A Colour Guide to Hong Kong Animals, Government Printer, Hong Kong.

499. Hill, J.E. and Carter, T.D. 1941. The mammals of Angola, Africa. Bulletin of the American Museum of Natural History 78(1): 1-211.

500. Hill, J.E. and Smith, S.E. 1981. Craseonycteris thonglongyai. Mammalian Species (American Society of Mammalogists) 160: 1-4.

501. Hill, W.C.O. 1970. Primates: comparative anatomy and taxonomy, Vol. 8: Cynopithecinae: Papio, Mandrillus, Theropithecus. Wiley and Sons, New York.

502. Hillman, C.N. and Clark, T.W. 1980. Mustela nigripes, Mammalian Species (American Society of Mammalogists) 126.

503. Hillman, J.C. 1982. Wildlife information booklet. Depart ment of Wildlife Management, Democratic Repuhlic of the Sudan Ministry of Wildlife Conservation and Tourism, Southem Region.

504. Hillman, J.C. 1986. Aspects of the biology of the Bongo Antelope Tragelaphus eurycerus Ogilby 1837 in south west Sudan. Biologicol Conservation 38: 255-272.

505. Hillman, K. 1983. The status of northem White Rhinos. IUCN/SSC African Elephant and Rhino Group Newsletter 1: 5-7.

506. Hillman-Smith, K. 1990. Rhino conservation in Garamba National Park. Pachyderm 13: 39-41.

507. Hills, D.M. 1979. Mammals previously unrecorded from the Sultanate of Oman, Arabia. Mammalia 43(4): 567-568.

508. Hines, C.J.H. 1990. Past and present distribution and status of the Wild Dog (Lycaon pictus) in Namibia. Nature et Faune 6(4): 29-38.

509. Ho Hua Chew 1988. The Dugong in Singapore waters. Malaysian Nat. 42(1): 22-25. 
510. Hoffman, R.K., Ponce del Prado, C.F. and Otte, K.C 1976. Registro de nos nuevas especies de mamiferos para el Peru, Odocoileus dichotomus (Illiger, 1811) y Chrysocyon brachyurus (Illiger, 1811) con notas sobre su habitat. Revista Forestal de Peru 6: 6-81

511. Homewood, K.M. 1975. Can the Tana Mangabey survive? Oryx 13(1): 53-59.

512. Homewood, K.M. and Redshaw, M. 1978. The ecology and conservation of the Tana Mangabey. The Dado 15: 32-38.

513. Homewood, K.M. and Rodgers, W.A. 1981. A previously undescribed mangabey from southern Tanzsnia. Intermational Joumal of Primatology 2: 47-55.

514. Honacki, J.H., Kinman, K.E. and Koeppl, J.W. 1982. Mammal species of the world, a taxonomic and geographic reference. The Association of Systematics Collections, Lawrence, Kansas.

515. Hoppe-Dominik, B. 1990. On the occurrence of the Honey-Badger (Mellivara capensis) and the viverrids in the Ivory Coast. Mustelid and Viverrid Conservation 3: 9-13.

516. Hom, A.D. 1987. The socioecology of the Black Mangabey (Cercocebus alerrimus) near Lake Tumba, Zaire. American Joumal of Primatology 12: 165-180.

517. Horrocks, J. 1984. The utilization and conservation of Vervets in Barbados. IUCN/SSC Primate Specialist Group Newslener 4: 30-31.

518. Horsten, F. 1982. Os parques nacionais e os outras zones de protecfao da natureza de Angola. Ministerio da Agricultura, Direç̧ao Nacional da Conservaç̧ao da Natureza, Luanda.

519. Horwich, R.H. 1980. Behavioral thythms in the Nilgiri Langur, Presbytis johnii. Primates 21(2): 220-229.

520. Horwich, R.H. 1984. Home range and food habits of the Nilgiri Langur, Presbyris johnii. Joumal of the Bombay Natural Histary Saciery 69(2): 255-267.

521. Horwich, R.H. 1984. The whooping display in Nilgiri Langurs: an example of daily fuctuations superimposed on 8 general trend. Primates 17(4): 419-431.

522. Horwich, R.H. and Johnson, E.D. 1984. Geographic distribution snd status of the Black Howler Monkey. IUCN/SSC Primate Specialist Group Newsletter 4: 25-27.

523. Hošek, E. 1984. [On occurrence and development of spreading of Common Otter in Moravia and Silesia]. Ochrana Prírody 5: 111-134. (In Russian).

524. Hoth, J., Velazquez, A., Romero, F.J., Leon, L., Aranda, M. and Bell, D.J. 1987. The Volcano Rabbil - a shrinking distribution and a threatened habitat. Oryx 21 : 85-91.

525. Howard, G.W. and Chabwela, H.N. 1987. The Red Lechwe of Busanga Plain, Zambia - a conservation success. Oryx $21: 233-235$.

526. Hubrecht, R.C. 1986. Operation Raleigh primate census in the Maya Mountains, Belize. Primate Conservation 7: 1517.

527. Hudson, W.S. and Wilson, D.E. 1986. Macroderma gigas. Mammalian Species (American Society of Mammalogists) 260.

528. Hufnagl, E. 1972. Libyan mammals. Oleander Press, Cambridge.

529. Hughes, R. 1982. Timely reappearance of a rare rat. Australian Natural History 20(10): 334
530. Hunkeler, P. 1978. Conservation du cerf de Barbsrie dans la forêt des Béni-Salah. Report to IUCN, Morges. Unpublished.

531. Huntley, B.J. 1974. Outlines of wildlife conservation in Angols. Joumal of the Southem African Wildlife Management Association 4: 157-166.

532. Husson, A.M. 1978. The mammals of Surinam. E.J. Brill, Leiden.

533. Hutterer, R. 1981. Crocidura manengubae n. sp., eine neue Spitzmaus aus Kamerun. Bonner Zoologische Beiträge 32: 241-248.

534. Hutterer, R. 1986. Disgnosen neuer Spitzmause aus Tanzania. Bonner Zoologische Beiträge 37: 23-33.

535. Hutterer, R. 1986. Eine neue Soricidengattung aus Zentrals frika . Zeitschrift fur Saugetierkunde 51: 257-266.

536. Hutterer, R. 1986. Synopsis der Gattung Paracrocidura (Mammalia: Soricidae), eine neuen Art. Bonner Zoologische Beiträge 37: 73-90.

537. Hutterer, R. and Dippenaar, N.J. 1987. A new species of Crocidura from Zambia. Bonner Zoologische Beiträge 38: 1-7.

538. Hutterer, R. and Dippenaar, N.J. 1987. Crocidura ansellorum, emended species name. Bonner Zoologische Beiträge 38: 269.

539. Hutterer, R. and Happold, D.C.D. 1983. The shrews of Nigeria. Bonner Zoologische Beiträge 18: 1-79.

540. Hutterer, R., Lopez-Jurado, L.F. and Vogel, P. 1987. The shrews of the eastem Canary Islands: a new species (Mammalia: Soricidae). Jaumal of Natural History 21: 1347-1357.

541. Idris, S.M.M. 1988. Elephants in Malaysia - is there a future? Tigerpaper 15(2): 14-18

542. Ilany, G. 1990. Leopard Panthera pardus in Israel. Pp. 45. In: Anon, Cat Specialist Group meeting reports. Cat News 12: 2-14.

543. Ilbl, A., Rahm, U.H. and Mathys, G. 1966. Les mammiferes et leurs tiques dans la forêt du Ruggege (République Rwandaise). Acta Tropica 23: 223-263.

544. Inskipp, T. and Corrigan, H. (Eds) 1992. A review of significant trade in animal species included in CITES Appendix 11. Detailed reviews of 24 priority species. Report of the CITES Animals Committee. CITES Secretariat, Lausanne.

545. IUCN. 1985. Mammals of South and Central America. Draft Document. IUCN Conservation Monitoring Centre, Cambridge.

546. Lawa, M. 1990. Iriomote Cat Felis iniomotensis. Pp. 1011. In: Anon, Cat Specialist Group meeting reports. Cal News 12:2-14.

547. Jachmann, H. 1984. Status of the Mwabvi thino (Diceros bicomis). Nyala 10(2): 77-90.

548. Jachmann, H. 1988. Numbers, distribution and movements of the Nazinga elephants. Pachyderm 10: 16-21.

549. Jackson, P. 1982. Elephants and hinos in Africa. A rime for decision. IUCN, Gland.

550. Jackson, P. 1984. The plight of cats. A summary report on the Cat Specialist Group workshop, Kanha National Park, India, 9-12. April 1984. Cat News 1: 1-15. 
551. Jackson, P. 1986. Chinese Tiger faces extinction. WWF Monthly Report June 1986: 137-141.

552. Jackson, P. and Ahlbom, G. 1988. A radio-telemetry study of the Snow Leopard (Panthera uncia) in West Nepal. Tigerpaper 15(2): 1-14.

553. Jackson, P. and Foose, T. 1990. Amur Leopard Panthera pardus orientalis. Pp. 3-4. In: Anon, Cat Specialist Group meeting reports. Cat News 12: 2-14.

554. Jaksic, F.M., Schlatter, R.P. and Yanez, J.L. 1980 Feeding ecology of central Chilean foxes, Dusicyon culpaeus and Dusicyon griseus. Joumal of Mammalogy 61:254-260.

555. Janzen, D.H. and Wilson, D.E. 1983. Mammals introduction. In: Jansen, D.H. (Ed.), Costa Rican Natural History. University of Chicago Press, Chicago, pp. 426-442.

556. Java, R.L. 1983. Census of wild ass (Equus hemionus khur). Tigerpaper 10(2): 23.

557. Jeannin, A. 1936. Les mammiferes sauvages du Cameroun. Lechevalier, Paris.

558. Jeffrey, S. 1982. Threats to the Proboscis Monkeys. Oryx 16(4): 337-339.

559. Jeffrey, S.M. 1970. Ghana's forest wildlife in danger. Oryx 10(4): 240-243.

560. Jeffrey, S.M. 1975. Ghana's new forest national park. Oryx 13(1): 34-35.

561. Jenkins, J.H. 1983. The status and management of the River Otter (Lutra conadensis) in North America. Acto Zoologica Fennica 174: 233-235

562. Jenkins, M.D. (Ed.) 1987. Madagascar. An environmental profile. IUCN, Gland.

563. Jenkins, P.D. 1984. Description of a new species of Sylvisorex from Tanzania. Bulletin of the British Museum of Natural History (Zoology) 47: 65-76.

564. Jenkins, P.D. 1988. A new species of Microgale (Insectivora: Tenrecidae) from northeastem Madagascor. American Museum Novitates 2910: 1-7.

565. Jenkins, P.D. and Hill, J.E. 1982. Mammals from Siberut, Mentawei lslands. Mammalia 46(2): 219-224.

566. Jenkins, S.H. and Busher, P.E. 1979. Castor canadensis. Mammalian Species (American Society of Mammalogists) 120: 1-8.

567. Jewell, P. 1990. Leopard Panthera pardus on ranches in Kenya. Pp. 5-6. In: Anon, Cat Specialist Group meeting reports. Cat News 12: 2-14

568. Johns, A. 1985. Current status of the southern Bearded Saki (Chiropotes satanas satanas). Primate Conservation 5: 28.

569. Johns, A. 1985. First field observations of Pithecia albicans. Primate Eye 26: 17-18.

570. Johns, A. 1986. Notes on the ecology and current status of the Buffy Saki, Pithecia albicans. Primate Conservation 7: 26-29.

571. Johns, A.D. and Ayres, J.M. 1987. Southern Bearded Sakis beyond the brink. Oryx 21: 164-167.

572. Johnsingh, A.J.T. 1990. Asiatic Lion Panthera leo persica. Pp. 7-8. In: Anon, Cat Specialist Group meeting reports. Cot News 12: 2-14.
573. Johnson, D.H., Ripley, S.D. and Thonglongya, K. 1980 Mammals from Nepal. Joumal of the Bomboy Natural History Society 77: 56-63.

574. Johnson, E. 1937. List of vanishing Gambian mammals. Joumal of the Society for the Preservation of the Fauna of the Empire (N.S.) 31: 62-66.

575. Johnson, J. Mangalraj 1978. The status, ecology and behaviour of Lion-tailed Macaque (Macaca silenus). Joumal of the Bombay Naturai History Society 75: 1017-1027.

576. Jolly, A. 1980. Frustrating search for the monkey with a lion's tail. Pp: 96-105. In: Prince Rainier and G.H Bourne (Eds), Primate conservation, Academic Press, New York.

577. Jolly, A. 1987. Priorités dans l'etude des populations de Lémuriens. In: Anon, Priorités en matière de conservation des espèces a Madagascar. IUCN, Gland.

578. Jones, C. 1969. Notes on ecologic al relationships of four species of lorisids in Rio Muni, West Africa. Folia Primotologica 11: 255-257.

579. Jones, C. and Anderson, S. 1978. Callicebus moloch Mammalian Species (American Society of Mammalogists) 112: $1-5$.

580. Jones, J.K. and Genoways, H.H. 1975. Dipodomys phillipsii. Mammalian Species (American Society of Mammalogists) 51: 1-3.

581. Jones, T.S. 1966. Notes on the commoner Sierra Leone mammals. Nigerian Field 31(1): 4-18.

582. Joslin, P. and Santiapillai, C. 1990. The Bomean Bay Cat Felis badia. Pp. 9-10. In: Anon, Cat Specialist Group meeting reports. Cat News 12: 2-14.

583. Joubert, E. 1973. Hahitat preference, distribution and status of the Hartmann Zebra Equus zebra hartmannae in South West Africa. Madoqua 1(7): 5-15.

584. Joubert, E. and Mostert, P.K.N. 1975. Distribution patterns and status of some mammals in South West Africa. Madoqua 9: 5-44.

585. Jouventin, P., Stahl, J.C. and Weimerskirch, H. 1982. La recolonisation des îles Crozet par les otaries (Arctocephalus tropicalis et A. gazella). Mammalia 46(6): 505-514.

586. Jungius, H. 1976. Project 960 Deer Island. International program for conservation of threatened species. World Wildlife Fund Yearbook 1975-76. WWF, Godalming. Pp. 201-217.

587. Kano, T. 1971. Distribution of the primates on the eastern shore of Lake Tanganyika. Primates 12: 281-304.

588. Kano, T. 1984. Distribution of Pygmy Chimpanzees (Pan paniscus) in the Central Zaire Basin. Folia Primatologica 43(1): 36-52.

589. Kappeler, M. 1981. The Javan Silvery Gibbon (Hylobates lar moloch), habitat, distribution, numbers. Unpublished.

590. Karami, M. 1992. Cheetah distribution in Khorasan Province, 1ran. Cat News 16: 4.

591. Karanth, K.V. 1986. A possible sighting record of the Malabar Civet (Viverra megaspila Blyth) from Kamataka. Joumal of Bombay Natural History Society 83: 192-193. 
592. Karr, J.R. 1973. Ecological and behavioural notes on the Liontailed Macaque (Macaca silenus) in South India. Journal of the Bombay Natural History Saciery 70(1): 191193.

593. Karstad, E.L. and Hudson, R.J. 1984. Census of the Mara River Hippopotamus (Hippopotamus amphibius), southwest Kenya, 1980-82. African Joumal of Ecalagy 22(2): 143-147.

594. Kavanagh, M. and Bennett, E.L. 1984. A synopsis of legislation and the primate trade in habitat and user countries. In: Mittermeier, R.A. and Mack, D. (Eds), The intemanional primate trade vol.1, TRAFFIC(U.S.A.), Washington, D.C., pp. 19-48.

595. Kavanagh, M. and Laursen, E. 1984. Breeding seasonality among Long-tailed Macaques, Macaca fascicularis, in Peninsular Malaysia. Intemational Joumal of Primatology 5(1): 17-29.

596. Keage, P.L. 1982. The conservation status of Heard Island and the McDonald Islands. University of Tasmania Environmental Studies Occasional Paper 13.

597. Kempf, C., Balestreri, A., Wotschikowsky, V. and Fernex, M. 1980. Chez nous, le lynx? Mythes et réalité. Les Guides Gesta, Paris.

598. Kenyon, K.W. 1982. Sea Otter (Enhydra tutris). In: Chapman, J.A. and Feldhamer, G.A. (Eds), Wild mammaLs of North America. J. Hopkins University Press, Baltimore, pp. 704-710.

599. Kerley, G.1.H. 1983. Record for the Cape Fur Seal Arctocephalus pusillus pusillus from Sub-antarctic Marion Island. South African Journal of Zoology 18(2): 139-140.

600. Kerley, G.I.H. 1983. Relative population sizes and trends, and hybridization of fur seals Arctocephalus tropicalis and A. gazella at the Prince Edward 1slands, Southern Ocean. South African Journal of Zoology 18(4): 388-392.

601. Khan, M.A.R. 1981. The non-human primates of Bangladesh. Tigerpaper 8(1): 12-15.

602. Khan, M.A.R. 1982. Wildlife of Bangladesh. University of Dhaka, Dhaka.

603. Khan, M.A.R. 1984. Endangered mammals of Bangladesh. Oryx 18(3): 152-156.

604. Khan, M.A.R. 1985. Mammals of Bangladesh. Nazma Reza, Dhaka.

605. Khan, M.A.R. and Ahsan, M.F. 1986. The status of primates in Bangladesh and a description of their forest habitats. Primate Conservation 7: 102-109.

606. Khan, M.K.b.M. 1978. Man's impact on the primates of Peninsular Malaysia. In: Chivers, D.J. and Lane-Petter, W. (Eds), Recent advances in primatology, val. 2: canservation, Academic Press, London, pp. 41-46.

607. Khan, M.K.M. 1985. Population and distribution of the Malayan Elephant, Elephas maximus in Peninsular Malaysia. Journal of Wildife and Parks 4: 1-16.

608. King, C.M. 1983. Mustela erminea. Mammalian Species (American Society of Mammalogists) 195.

609. King, C.M. (Ed). 1990. The handbook of New Zealand mammals. Oxford University Press, Aukland.

610. King, J.E. 1983. Seals of the warld. (2nd edition). British Museum (Natural History), London.

611. Kingdon, J. 1971. East African mammals. An atlas of evolurion in Africa. Vol.l. Academic Press, London.
612. Kingdon, J. 1974. East African mammals. An atlas of evolusion in Africa. Vol. (I pan A (Insecrivores and Bats). Academic Press, London.

613. Kingdon, J. 1977. East African mammals. An atlas of evalution in Africa. Vol. IIIA. Academic Press, London.

614. Kingdon, J. 1982. East African mammals. An atlas of evalution in Africa. Val. II part B (Hares and Rodents). Academic Press, London.

615. Kingdon, J. 1982. East African mammals. An atlas of evolurion in Africa. Vol. III pan C (Bovids). Academic Press, London.

616. Kinnaird, M.F. 1985. Aerial census of manatees in northeastern Florida. Biological Conservation 32(1): 59-79.

617. Kirkpatrick, R.D.L. and Cartwright, A.M.L. 1975. List of mammals known to occur in Belize. Biotropica 7(2): 136-140.

618. Kleiman, D. (Ed.) 1978. The bialogy and conservation of the Callithrichidae. Smithsonian Institution, Washington, D.C.

619. Klingel, H. 1972. Somali Wild Ass status survey in the Danakil Region, Ethiopia. Final repon WWF Project 496.

620. Klinowska, M. 1991. Dolphins, parpoises and whales of the world: the IUCN red data book. IUCN, Gland and Cambridge.

621. Kock, D. 1969. Die verbreitung der primaten in Sudan. Zeitschrift fur Saugerierkunde 34: 193-216.

622. Köhncke, M. and Leonhardt, K. 1986. Cryptopracta ferax. Mammalian Species (American Society of Mammalogists) 254.

623. Kolstad, M., Mysterud, I., Kvam, T., S $\phi$ renson, O.J. and Wikan, S. 1986. Status of the Brown Bear in Norway: distribution and population 1978-82. Bialagical Conservation 38: 79-99.

624. Konstant, W., Mittermeier, R.A. and Nash, S.D. 1985. Spider monkeys in captivity and in the wild. Primate Conservation 5: 82-109.

625. Koopman, K.F. 1982. Biogeography of the bats of South America. In: Maros, M.A. and Genoways, H.H. (Eds), Mammalian biology in South America. Pymatuning Laboratory of Ecology, University of Pittsburgh, Special Publication, Pittsburgh, pp. 273-302.

626. Koster, S. 1981. A survey of the vegetation and large mammals of Park W, Niger. M.Sc. Thesis, Michigan State University, Michigan.

627. Kramer, R.J. 1971. Hawaiian land mammals. Charles E. Tuttle Company.

628. Kuhn, A.J. 1965. A provisional checklist of the mammals of Liberia. Senckenbergiana Bialagica 46: 321-340.

629. Kumerloeve, H. 1975. Die saugetiere (Mammalia) der Turkei. Veraffentlichungen der Zaalogischen Staakssammlung Munchen 18: 69-158.

630. Kumerloeve, H. 1975. Die saugetiere (Mammalia) Syriens und des Libanon. Veroffentichungen der Zoalogischen Staatssammlung Munchen 18: 159-225.

631. Kunhunu, N.V.A. 1991. Status der Malabar-Zibetkatze und des Jerdon-Musangs in den West Ghats. Zoolagische Gesellschafi Anen und Populationsschutz 7(1): 3-4. 
632. Kunz, T.H. and Martin, R.A. 1982. Plecolus townsendii. Mammalian Species (American Society of Mammalogists) 175: $1-6$.

633. Kuper, J.H. 1978. The wild asses at the Little Rann of Kutch. Tigerpaper 5(3): 29-32.

634. Kurt, F. 1978. Kashmir Deer (Cervus elaphus hanglu) in Dachigam. In: Anon, Threatened deer. Proceedings of a working meeting of the Deer Specialist Group of the SSC. IUCN, Morgea, pp. 87-108.

635. Kurten, B. 1968. Pleistocene mammals of Europe. Aldine, Chicsgo.

636. Lamarche, B. 1980. L'addax Addax nasomaculatus (Blainville); biologie. Report to WWF/IUCN, Gland. Unpublished.

637. Lamhna, E.N. 1979. Provisional distribution aslas of amphibians, reptiles and mammals in treland. (2nd edition). An Foras Forbartha, Dublin.

638. Lamotte, M. 1942. La faune mammalogique du Mont Nimba. Mammalia 3(4): 114-119.

639. Lamprey, H. 1975. Repon on the desert encroachment reconnaissance in northem Sudan. Report to IUCN and UNEP. Unpublished

640. Langguth, A. 1975. Ecology and evolution in the South American canids. In: Fox, M.W. (Ed.), The wild canids, their systemaric behavioural ecology and evolution. Van Nostrand Reinhold, New York, pp. 192-206.

641. Largen, M.J. and Yalden, D.W. 1987. The decline of Elephant and Black Rhinoceros in Ethiopia. Oryx $2 \mathrm{I}: 103$ 106 .

642. Laurent, C. 1990. The Mammals of Laos and the hunting practices. Vientiane.

643. Laurie, E.M.O. and Hill, J.E. 1954. List of land mammats of New Guinea, Celebes and adjacent islands, 1758-1952. British Museum (Natural History), London.

644. Laurie, W.A., Lang, E.M. and Groves, C.P. 1983. Rhinoceros unicomis. Mammalian Species (American Society of Mammalogists) 211: 1-6.

645. Lovieren, L. P. van and Bosch M. L. 1977. Evaluation des densités de grands mammiferes dans le Parc National de Bouba Ndjida, Cameroun. La Terre et la Vie 31: 3-32.

646. Lay, D.M. 1967. A study of the mammals of Iran. Fieldiana Zaology 54: 1-282.

647. Lear, R.J. and Bryden, M.M. 1980. A study of the Boulenose Dolphin Tursiops truncatus in eastem Australian waters. Canberra, ANPWS, Occasional Papers No.4.

648. Leatherwood, S., Hubbs, C.L. and Fisher, M. 1979. First records of Risso's Dolphin (Grampus griseus) from the Gulf of California with detailed notes on mass stranding. Transactions of the San Diego Sociery for Nalural History 19(3): 45-52.

649. Lee, P.C. Thomback, J. and Bennett, E.L. 1988. Threatened primales of Africa. The IUCN red dala book. IUCN, Gland and Cambridge.

650. Leskes, A. and Acheson, N.H. 1970. Social organization of a free-ranging troop of Black-and-white Colobus Monkeys (Colobus abyssinicus). Proceedings of the 3rd Intemational Congress of Primatology, Zurich 1970, 3: 22 31.

651. Lever, C. 1983. Wildlife conservation in the southern Sudan. Oryx 17(4): 190-193.
652. Lewis, J.G. and Wilson, R.T. 1979. The ecology of Swayne's Hartebeest. Biological Conservation 15: 1-12.

653. Lewis, R.E., Lewis, J.H. and Atallah, S.1. 1968. A. review of Lebanese mammals, Camivora, Pinnipedia, Hyracoidea and Artiodactyla. Joumal of Zoology, London 154: $517-531$.

654. Li Zhi-xiang and $\mathrm{Ma}$ Shi-lai 1980. A revision of the White-headed Langur. Acta Zaotaxonomica Sinica 5(4): $440-442$.

655. Li Zhi-xiang, Ma Shi-lai, Hua Cheng-hui and Wang Yingxiang 1981. The distribution and habits of the Yunnan Golden Monkey, Rhinopithecus bieti. Joumal of Human Evolution 11(7): 633-638.

656. Lidicker, W.Z. 1989. Rodents. A world survey of species of conservation concem. IUCN, Gland.

657. Ligon, S. 1976. Aerial survey of the Dugong in Kenya FAO ACMRR/MM/SC/107. Scientific Consultation on Marine Mammals, Bergen, Norway, 31 August - 9 September 1976.

658. Liles, G. and Jenkins, L. 1984. A field survey for Otters (Lutra lutro) in Yugoslavia. Joumal of Zoology, London 203: 282-284.

659. Lindsay, N.B.D. 1980. A report on the field study of Geoffroy's Tamarin Saguinus oedipus geoffroyi. Dodo 17: 27-51.

660. Locati, M. 1990. The Goral of Binsar Wildlife Sanctuary, northern India. Caprinae Specialist Group News 5: 6-7.

661. Lönberg, E. 1913. Mammals from Ecuador and related forms. Arkiv for Zoologi 8(16): 1-36.

662. Lorini, M.L. and Persson, V.G. 1990. New species of Leonlopithecus Lesson 1840 from Southern Brazil (Primstes: Callitrichidae). Bol. Mus. Nac. Rio J. Zool. (338): $1-14$.

663. Loughlin, T.R. and Perez, M.A. 1985. Mesoplodon stejnegeri. Mammalian Species (American Society of Mammalogists) 250: 1-6.

664. Lovari, \$. 1977. The Abnuzzo Chamois. Oryx 14(1): 47-50.

665. Lovari, S. (Ed.) 1985. The biology and management of mounlain ungulates. Croom Helm, London.

666. Lovari, S. 1990. Some notes on the wild ungulates of the Sagarmatha National Park, Khumba Himal (Nepal). Caprinae Specialist Group News 5: 2-4.

667. Lu Ho-gee 1984. Fea's Muntjac discovered for the first time in China. Oryx 18(1): 5 .

668. Lu Ho-gee and Sheng He-lin 1984. Status of the black Munlisc, Muntiacus crinjfrons in eastern China. Mammal Review 14(1): 29-36.

669. Lu, Hou-ji 1990. Cat problems in China. P. 10. In: Anon, Cat Specialist Group meeting reports. Cat News 12: 2-14.

670. Luna, M.L. 1982. Conservation of the Yellow-tailed Woolly Monkey. Intemational Zoo Yearbook 22: 47-52.

671. Ma Yi-ching 1983. The status of bears in China. Acta Zoologica Fennica 174: 165-166

672. Macdonald, D.M. 1984. Encyclopaedia of mammals: vol. I camivores, seals, whales, dolphins, primales. George, Allen and Unwin, London, pp. 1-447. 
673. Macdonald, D.W. 1982. Notes on the size and composition of groups of Proboscis Monkey, Nasalis larvatus. Folia Primatologica 37: 95-98.

674. Macdonald, S.M. 1983. The status of the Otter (Lutra lutra) in the British Isles. Mammal Review 13(1): 11-23.

675. Macdonald, S.M. and Mason, C.F. 1982. Oners in Greece. Oryx 16(3): 240-244.

676. Macdonald, S.M. and Mason, C.F. 1982. The Otter (Lutra luera) in central Portugal. Biological Canservation 22: 207-215.

677. Macdonald, S.M. and Mason, C.F. 1983. The Otter (Lutra lutra) in southern Italy. Biological Conservation 25: 95-101.

678. Macdonald, S.M. and Mason, C.F. 1983. The Otter (Lutra lutra) in Tunisia. Mammal Review 13(1): 35-37.

679. Macdonald, S.M. and Mason, C.F. 1984. Otters in Morocco. Oryx 18(3): 157-159.

680. Macdonald, S.M. and Mason, C.F. 1985. Onters, their habitat and conservation in northeast Greece. Biological Conservation 31(3): 191-210.

681. Macdonald, S.M. and Mason, C.F. 1986. Otters of the Jordan Valley. WWF Monthly Repon June 1986: 149-153.

682. Macdonald, S.M., Mason, C.F. and Shalmon, B. 1986. A survey for Otters in Israel. Oryx 20: 233-236.

683. MacKenzic, A.F. 1952. Economic problems of the monkey population in Sierra Leone. Praceedings of the Zoological Society of London 122(2): 511.

684. Mackinnon, J. 1983. Report on a visil to Hanoi, 18-25 November 1983, to participate in the workshop of the programme on natural resources and environmental research and protection. Unpublished.

685. Mackinnon, J. 1986. Bid to save the Kouprey. WWF Monthly Report April 1986: 91.97.

686. Mackinnon, K.S. 1983. Consultancy to Indonesia to determine population estimates of the Cynomologus or Long-tailed Macaque Macaca fascicularis (and other primates) and the feasibility of semi-wild breeding projects of this species. Repon of W.H.O

687. Mahdi. N. and Georg, P.V. 1969. A systematic list of the vertebrates of 1raq. Iraq Natural History Musenm Publication 26: 1-77.

688. Mahoney, J.A. and Richardson, B.J. 1988. Muridae. In Walton, D.W. (Ed.), Zoological Catalogue of Australia S(Mammalia): 154-192.

689. Malbrant. R. 1952. Faune du Centre Africain Français (mammiferes el oiseaux). Lechevalier, Paris

690. Malbrant, R. and Maclatchy, A. 1949. Colobus polykomas. In: Malbrant, R. and Maclatchy, A., Encyclopedia Biologique, Lechevalier, Paris, pp. 216-217.

691. Malbrant, R. and Maclatchy, A. 1949. Faune de l'equateur Africain Français. Vol. 2. mammiferes. Encyclopédie Biologique 36. Lechevalier, Paris.

692. Mallinson, J. 1984. Lion tamarins' survival hangs in balance. Onx 18(2): 72-78

693. Mallon, D. 1983. The status of Ladakh Urial Ovis orientalis vignei in Ladalh, India. Bialogical Conservation $27(4)$ : $373-381$

694. Mallon, D. 1988. Snow Leopards in northern Hunza. Cat News 9: 12
695. Mallon, D.P. 1985. The mammals of the Mongolian People's Republic. Mammal Review 15: 71-102.

696. Mann, G. 1950. Nuevos mamiferos de Tarapaca. Resultados de la expedicion Universitaria a Tarapaca, 1948. Investigacianes Zoologicas Chilenas 1(2): 4-6.

697. Mantecon, M.A.F. de, Mudry de Pargament, M.D. and Brown, A. 1984. Cebus apello de Argentina, distribucion geografica, fenotipo y cariotipo. Revista del Musea Argentino de Ciencias Naturales 'Bemardino Rivadavia', Zoalogia 13: 399-408.

698. March, E.W. 1957. Gorillas of eastern Nigeria. Oryx 4: 30-34.

699. Mares, M.A., Deja, R.A. and Barquez, R.M. 1989. Guide to the mammals of Salta Province, Argentina. University of Oklahoma Press.

700. Mares, M.A. and Genoways, H.H. 1982. Mammalian bialogy in South America. Vol. 6: Special Publication Series. Pymatuning Laboratory of Ecology, University of Pittsburgh, Pittsburgh.

701. Mares, M.A., Ojeda, R.A. and Kosco, M.P. 1981 Observations on the distribution and ecology of the mammals of Salta Province, Argentina. Annals of the Camegie Museum 50: 151-206.

702. Mares, M.A., Willig, M.R., Streilein, K.E. and Lacher, T.E. 1981. The mammals of northeast Brazil - a preliminary assessment. Annals of the Carnegie Museum 50: $81-137$.

703. Marineros, L. and Gallegos, F.M. 1988. Mamiferos silvestres de Honduras. Asociacion Hondureña de Ecologia.

704. Marker-Koaus, L. and Koaus, D. 1990. Status of the Cheetah in Zimbabwe and Namibia. Cat News 12: 15-16.

705. Marsden, S. 1983. Rehabilitating Chimpanzees in Gambia. Fund for Animals (Australia) Newsletter 3(2): 14-15.

706. Marsh, C. 1986. A resurvey of Tana River primates and their habitat. Primate Conservation 7: 72-82.

707. Marsh, C.W. and Wilson, W.L. 1981. A survey of primates in Peninsular Malaysian forests. Universiti Kebangsaan, Malaysia and University of Cambridge, UK.

708. Marsh, H. 1983. Conserving the Dugong (cowfish) in Vanuatu. Naika 9: 1-5.

709. Marshall, P. 1967. Wild mammals of Hong Kang. Oxford University Press, Oxford.

710. Martens, V. 1989. Otter survey in the northern part of the Netherlands. IUCN Ouer Specialist Group Bulletin 4: 27 28.

711. Marin, E.B., Martin, C.B. and Vigne, L. 1987. Conservation crisis - the hinoceros in India. Oryx 21 : 212-218.

712. Mashkin, V.I. 1984. [Contemporary range and numbers of Mamata menzbieri Kaschkarov, 1925, in west Tian Shan.] Byulleten ' Moskouskogo Obshchestva Ispytatelei Priordy, Biol. 89(1): 33-43 (In Russian).

713. Maynes, G.M. 1982. A new species of rock wallaby Petrogale persephone from Prosperine, central Queensland. Aistralian Mammalogy 5: 47-58.

714. Mazak, V. 1981. Panthera tigris. Mammalian Species (American Society of Mammalogists) 152: 1-8. 
715. McCarthy, T.J. 1982. Chironectes, Cyclopes, Cabassous and probably Cebus in southern Belize. Mammalia 46(3): $397-400$.

716. McCarthy, T.J. 1983. Mammals of Belize. Belize Audubon Saciery Bulletin 15(4): 1-3.

717. McFarlane, D.A. 1983. Phyllonycieris aphylla IUCN red data book inventory repon form. Natural History Museum Los Angeles County, Los Angeles, 23 August 1983.

718. McGrew, W.C., Baldwin, P.J. and Tutin, C.E.G. 1981. Chimpanzees in a hol, dry and open habitat: Mt. Assirik, Senegal, West Africa. Jaumal of Human Evalurian 10: 227-244.

719. Mead, J.G. 1981. First records of Mesoplodon hecton (Ziphiidae) from the northern hemisphere and a description of the adult male. Joumal of Mammalogy 62(2): 430-432.

720. Mech, L.D. 1974. Canis lupus. Mammalian Species (American Society of Mammalogists) 37.

721. Medway, Lord 1969. The wild mammals af Malaya and Singapore. Oxford University Press, Oxford.

722. Medway, Lord 1970. The monkeys of Sundaland: ecology and systematics of the cercopithecids of a humid equatorial environment. In: Napier, J.R. and Napier, P.H. (Eds), OLd Warld Mankeys, Academic Press, New York, pp. 513-544.

723. Medway, Lord 1977. Mammals of Borneo. Managraphs of the Malaysian Branch of the Royal Asiatic Society 7: 1-172.

724. Meester, J. and Setzer, H.W. (Eds) 1971. The mammals of Africa. An identification manual. Smithsonian lnstitution Press, Washington, D.C.

725. Meester, J.A.J. 1976. South African red data book-small mammals. Sauth African National Scientific Programmes Report No. 11, National Scientific Programmes Unit, Council for Scientific and Industrial Research.

726. Meier, B., Albignac, R., Peyrieras, A., Rumpler, Y. and Wright, P. 1987. A new species of Hapalemur (Primates) from south-east Madagascar. Folia Primatologica 48: 211 215.

727. Melendres, A.P. 1978. Notas sobre las nutrias de Rio del Ecuador. In: Duplaix, N. (Ed.), Otters. Proceedings of the first working meeting of the IUCN/SSC Otter Specialist Group, Paramaribo, Suriname 27-29 March 1977. IUCN, Morges, pp. 56-62.

728. Mens, R.A. 1978. Fauna y caza en Costo Rica. Lil. S.A. San José, Costa Rica.

729. Ménard, N., Amroun, M., Said, R.M. and Gautier-Hion, A. 1986. Status of the Barbary Macaque (Macaca sylvanus) in Tikdja Forest, Algeria. Primate Canservation 7: 35-38.

730. Mendelssohn, H. 1983. Conservation of the Wolf in Israel. Acra Zoologica Fentica 174: 281-282.

731. Mendelssohn, H. 1983. Status of the Wolf in the Middle East. Acta Zoologica Fennica 174: 279-280.

732. Mendelssohn, H. 1989. Felids in Israel. Cat News 10:2-4.

733. Mendelssohn, H. 1990. Anatolian Leopard Panthera pardus tulliana. P. 4. In: Anon, Cat Specialist Group meeting reports. Cal News 12: 2-14.

734. Mendelssohn, H. and Yom-tov, Y. 1987. Plants and animals of the land of Israel, 7. Mammals. Ministry of Defence Publishing House and Society for Protection of Nature, lsrael.
735. Menkhorst, P.W. and Seebeck, J.H. 1981. The distribution, habitat and status of Pseudomys fumeus Brazenor (Rodentia: Muridae). Australian Wildlife Research 8(1): 87-96.

736. Mensch, P.J.A. van and Bree, P.J.H. van 1969. On the African Golden Cat, Profelis aurata Temm. Biologia Gabonica 5: 235-269.

737. Menzies, J.1. 1970. An eastward extension to the known range of the Olive Colobus Monkey (Colobus verus van Beneden). Jaumal of the West African Science Associatian 15: $83-84$.

738. Menzies, J.1. and Pernetta, J.C. 1986. A taxonomic revision of cuscuses allied to Phalanger orientalis. Joumal of the Zoological Society of London 1986(B): 551-618.

739. Merz, G. 1986. The status of the Forest Elephant Loxodonta africana cyclotis, Matschie, 1900, in the Gola Forest Reserves, Sierta Leone. Biological Conservation 36: $83-94$.

740. Merz, G. and Hoppe-Dominik, B. 1991. Distribution and status of the Forest Flephant in the lvory Coast, West Africa. Pachyderm 14: 22-24

741. Mickevicius, E. and Baranauskas, K. 1992. Status, abundance and distribution of mustelids in Lithuania.

742. Mickleburgh, S.P., Hutson, A.M., and Racey, P.A. 1992. Old World fruit bats an action plan for their conservation. IUCN, Gland.

743. Miller, B.J., Anderson, S.H., Don Carlos, M.W. and Thorne, E.T. 1988. Biology of the endangered Blackfooted Ferret and the role of captive propagation in its conservation. Canadian Joumal of Zoology. 66: 765-773.

744. Miller, F.W. 1930. Notes on some mammals of Southem Mato Grosso, Brazil. Joumal of Mammalogy 11: 10-23.

745. Miller. G.S. and Kellogg. R. 1955. List of North American recent mammals. Smithsonian Institution Press, Washington, D.C.

746. Miller, S.D., Rottmann, J., Raedeke, K.J. and Taber, R.D. 1983. Endangered mammals of Chiis: status and conservation. Biological Conservation 25: 335-352.

747. Mills, M.G.L. 1982. Hyaena bnunnea. Mammalian Species (American Society of Mammalogists) 194: 1-5

748. Mills, M.G.L. 1990. Lion Panthera leo and Cheetah Acinanyx jubatus in Kruger National Park, South Africa. P. 6. In: Anon, Cat Specialist Group meeting reports. Cat News 12: 2-14.

749. Mills, S. 1983. Norway's last Wolves. Wildlife (London) $25(5): 190$

750. Mills, S. 1987. Does Sweden have room for its Wolves? Oryx $21:$ 92-96.

751. Milton, K. and Lucca, C. de 1984. Population estimate for Brachyteles at Fazenda Barreiro Rico, Sao Paulo State, Brazil. IUCN/SSC Primate Specialist Group Newsletter 4: 27-28.

752. Mitchell, A. 1983. Conservation of the Simalur lsland Macaque, Indonesia. IUCN/SSC Primate Specialist Group Newsletter 3: 32-33

753. Mitchell, E. 1975. Porpoise, dolphin and small whale fisheries of the world. IUCN Monograph No. 3. IUCN, Morges. 
754. Mitchell, E.R. and Reeves, R.R. 1982. Factors affecting abundance of Bowhead Whales Balaena mysricetus in the Eastem Arctic of North America, 1915-1980. Biological Canservation 22(1): 59-78.

755. Mitchell, R. and Punzo, F. 1976. New mammal records from Nepal. Joumal of the Bombay Natural History Saciety $73: 54-58$

756. Mitchell, R.M. 1975. A checklist of Nepalese mammals. Säugerierkundliche Mitteilungen 23: 152-157.

757. Mittermeier, R.A. and Coimbra-Filho, A.F. 1983 Distribution and conservation of New World primate species used in biomedical research. In: Heam, J. (Ed.), Reproduction in New World Primates. MTP Press, Lancaster.

758. Mittermeier, R.A., Coimbra-Filho, A.F., Constable, 1.D., Rylands, A.B. and Valle, C. 1982. Conservation of primates in the Atlantic forest region of eastern Brazil. Intemational Zoo Yeorbook 22: 2-17.

759. Mittermeier, R.A. and Oates, J.F. 1985. Primste diversity: the world's top countries. Primate Conservarion 5: 41-48.

760. Mittermeier, R.A. and Roosmalen, M.G.M. van 1982. Conservation of primates in Surinam Intemarional Zoo Yearbook. 22: 59-68.

761. Mochi, U. and Carter, T.D. 1974. Hoofed mammols of the world. Lutterworth Press, London.

762. Mohnot, S.M. 1978. The conservation of non-human primates in India. In: Chivers, D.J. and Lane-Petter, W. (Eds), Recent advances in primatology, vol. 2 , conservation. Academic Press, London, pp. 47-53

763. Mohnol, S.M. 1980. On the primate resources of India. Joumal of the Bombay Notural History Sociery 75: 961970.

764. Monard, A. 1983. Résultats de la mission scientifique du Dr Monard en Guinée Portugaise. Arq. Mus. Bocage 9: 121-150

765. Monath, T.P. and Kemp, G.E. 1973. Importance of non-human primates in yellow fever epidemiology in Nigeria. Tropical and Geogrophical Medecine 25: 28-38.

766. Mondolfi, E. 1976. Fauna silvestre de los bosques humedos de Venezuela. Sierra Club - Consejo de Bienestar Rural.

767. Mondolfi, E. and Eisenberg, J.F. 1979. New records for Ateles belzebuth hybridus in Northern Venezuela. in: Eisenberg, J.F. (Ed.), Vertebrate ecology in the northem Neotropics. Smithsonian, Washington, D.C.

768. Monfort, A. and Monfort, M. 1973. Les grands mammiferes du Parc National de Taj, Côte d'Jvoire. La Terre et la Vie 4: 499-506.

769. Montfor, N. 1985. Les mommiferes du Rwando. Rotary Club de Kigali.

770. Morales, R.G. 1979. Lista de mamiferos Centroamericanos. CATIE, Departamento de Ciencias Forestales. Unpublished.

771. Moreau, R.E. and Pakenham, R.H.W. 1940. The land vertebrates at Pemba, Zanzibar and Mafia: a zoogeographical study. Proceedings of the Zoologicol Sociery of London, Series A. 110: 97-128.

772. Morris, R., Morris, D. and Barzdo. J. 1981. The Giant Panda. Macmillan, London.
773. Morrison, J.L. 1992. Persistence of the meadow jumping mouse Zapus hudsonius luteus in New Mexico. Southwestem Naturalist 37(3): 308-311.

774. Moss, C. 1990. Elephants in Tarangire. Pachyderm 13: 26-30.

775. Mountfon, G. 1981. Saving the Tiger. Michael Joseph, London.

776. Muckenhirn, N.A. and Eisenberg, J.F. 1978. The status of primates in Guyana and ecological correlations for neotropical primates. In: Chivers, D.J. and Lane-Petter, W. (Eds), Recent advances in primotology, vol. 2, conservarion. Academic Press, London, pp. 27-30.

777. Müffling, S. von 1977. Fischouter in Europa. Loizenkirchen.

778. Mukheriee, R.P. 1982. Phayre's Leaf Monkey (Presbyris phayrei Blyth, 1847) of Tripura. Joumol of the Bombay Natural History Society 79(1): 47-56.

779. Muriuki, J.W. and Tsingalia, M.H. 1990. A new population of de Brazza's Monkey in Kenya. Oryx 24: $157-162$.

780. Mumyak, D.F. 1981. Censusing the Gorillas in Kahuzi-Biega National Park. Biological Conservarion 21(3): 163-176

781. Muskin, A. 1984. Field notes and geographic distribution of Callithrix aurita in eastem Brazil. American Joumal of Primotology 7(4): 377-380.

782. Musser, G.G. and Dagosto. M. 1987. The identity of Tarsius pumilus. American Museum Novitates 2867: 1-53.

783. Musser, G.G., Koopman, K.F. and Cafifia, D. 1982. The Sulawesian Pleropus arquatus and $\boldsymbol{P}$. argentatus are Aceradon celebensis; the Philippine $P$. leucotis is an Aceradon. Joumal of Mammalogy 63: 319-328.

784. Myers, N. 1976. The Leopard Panthero pordus in Africa. IUCN Monograph No.5. IUCN, Morges.

785. Nader, I.A. 1984. A second record of the Caracal Lynx Caracal caracal schmitzi (Matschie, 1912) for Saudi Arabia (Mammalia: Camivora). Mammalia 48(1): 148-150.

786. Nader, I.A. and Büttiker, M. 1980. Mammalia: Fam. Canidae. Records of the Arabian Wolf, Canis lupus arabs Pocock, 1934, from Saudi Arabia. Fauna of Saudi Arabia 2: $405-411$.

787. Nagorsen, D.W. 1987. Marmota vancouverensis. Mommolian Species (American Society of Mammalogists) 270: $1-5$.

788. Napier, P.H. 1976. Calalogue of primates in the British Museum (Natural History), par I, families Callithrichidae and Cebidae. British Museum (Natural History), London.

789. Napier, P.H. 1981. Cotologue of primates in the British Museum (Natural History) and elsewhere in the Brinsh lsles, part II, family Cercopithecidae, subfamity Cercopithecinae. British Museum (Natural History), London.

790. Napier, P.H. 1985. Catalogue of primates in the British Museum (Nalural History) and elsewhere in the Brinish Isles, part III, family Cercopithecidae; suhfamity Colobinae. British Museum (Natural History), London.

791. Napier, P.H. and Napier, J. 1967. A handbook of living primates. Academic Press, London. 
792. Nash, S.V. and Nash, A.D. 1985. The status and ecology of the Sumatran Elephant (Elephas maximus sumasranus) in the Padang Sugihan Wildlife Reserve, South Sumatra. WWF/IUCN 3133 PS/Final Report (in part).

793. Naumann, C. and Niethammer, J. 1973. Zur Saügetierfauna des afghanischen Pamir und des Wakhan. Bonner Zoologische Beitrage 24: 237-248.

794. Naurois, R. de 1969. Notes brèves sur l'avifaune de l'Archipel du Cap Vert, faunistique, endémisme, écologie. Bulletin de l'Insritut Fondomental d'Afrique Noire. 31: 143-218.

795. Nauroz, K.M. and Naumann, C.M. 1975. Bemerkungen zur Verbreitung des Markhors, Capra falconeri (Wagner, 1839) in Afghanistan. Säugetierkundliche Mitteilungen 23: 81-85.

796. Navarro, L.D. 1985. Status and distribution of the Ocelot in South Texas. MSc. thesis, Texas A and I University. Unpublished.

797. Nawaz, M. 1983. The endangered mammals of Pakistan. Tigerpaper 10(3): 15-20.

798. Neas, J.F. and Hoffmann, R.S. 1987. Budorcas taxicolor. Mammalian Species (American Society of Mammalogists) 277: $1-7$.

799. Nellis, D.W. 1989. Herpestes auropunctatus. Mammalian Species (American Society of Mammalogists) 342.

800. Neve, P. 1973. Bryde's Whale beached. Joumal of the Saudi Arabian Natural History Sociery 7: 14.

801. Newby, J. 1970. The ecological resources of the Ouadi Rimé - Ouadi Achim Faunal Reserve, Chad. UNDP/FAO Wildlife Conservation and Management Project CHD/69/004. Unpublished.

802. Newby, J. 1975. The Addax and Scimitar-horned Oryx in Niger. Report to IUCN, Morges. Unpublished.

803. Newby, J. 1975. The Addax and the Scimitar-homed Oryx in Chad. Report to IUCN, Morges. Unpublished.

804. Newby, J. 1981. The conservation status of the Sahelo-Saharan fauna of Africa. Report to IUCN/WWF, Gland. Unpublished.

805. Newby, J.E. 1982. Avant-projet de classement d'une aire protégée dans l'Air et le Ténéré (République du Niger). Repon to IUCN/WWF, Gland. Unpublished.

806. Newby, J.E. and Jones, D.M. 1980. An ecological survey of the Takolokouzet Massif and surrounding area in the Eastem Air Mountains Republic of Niger. Government of the Republic of Niger/ Zoological Society of London/ IUCN/ Quest 80s Lid/ Fauna and Flora Preservation Society.

807. Nguyen Xuan Dang, Pham Trong Anh and Dang Huy Huynh 1992. The biology and status of Owston's Palm Civet in Vietnam. Small Camivore Conservation 6: 5-6.

808. Nicoll, M.E. and Rathbun, G.B. 1990. African Insectivora and Elephant-shrews. An action plan for their conservation. IUCN, Gland.

809. Nicoll, M.E. and Suttie, J.M. 1982. The Sheath-tailed Bat, Coleura seychellensis (Chiroptera: Emballonuridae) in the Seychelles Islands. Joumal of Zoology, London 197: 421426.

810. Niemitz, C. 1979. Results of a field study on the Western Tarsier (Tarsius bancanus bomeanus Horsfield, 1821) in Sarawak. Sarawak Museum Joumal 27(48): 171-228.
811. Niethammer, G. 1970. Beobachtungen am Pyrenaen-Desman, Galemys pyrenaicus. Bonner Zoolische Beiträge 21: 157-182.

812. Nievergeh, B. 1981. Thexes in an African environment. Ecological Stududies Analysis and synthesis 40: 1-77.

813. Nishiwaki, M., Yamaguchi, M., Shokita, S., Uchida, S. and Kataoka, T. 1983. Recent survey on the distribution of the African Manatee. The Scientific Reports of the Whales Research Insritute 34: 137-147.

814. Noriyuki Ohtaishi and Yaoting Gao. 1990. A review of the distribution of all species of deer (Tragulidae, Moschidae and Cervidae) in China. Mammal Review 20(2): 1-20.

815. Nowell, K. 1990. Clouded Leopard Neofelis nebulosa and wildlife trade in Taiwan. P. 9. In: Anon, Cat Specialist Group meeting reports. Cat News 12: 2-14.

816. O'Connor, S., Pidgeon, M. and Randria, Z. 1986. Conservation program for the Andohahela Reserve, Madagascar. Primate Conservation 7: 55-57.

817. O'Gara, B.W. 1978. Antilocapra americana. Mammalian Species (American Society of Mammalogists) 90: 1-7.

818. O'Leary, H. 1993. Monkey business in Gibraltar. Oryx 27: 55-57.

819. Oates, J.F. 1969. The lower primates of eastem Nigeria. African Wildlife 23: 321-332.

820. Oates, J.F. 1977. The Guereza and man: how man has affected the distribution and abundance of Colobus guereza and other black colohus monkeys. In: Rainier, Prince and Boume, G.H. (Eds), Primate conservation. Academic Press, New York, pp. 420-467.

821. Oates, J.F. 1979. Comments on the geographical distribution and status of the South Indian Black-leaf Monkey (Presbytis johnii). Mammmalia 43(4): 485-493.

822. Oates, J.F. 1982. In search of rare forest primates in Nigeria. Onyx 16(5): 431-436.

823. Oates, J.F. 1985. IUCN/SSC Primate Specialist Group action plan for African primale conservation: 1986-90. IUCN, Gland.

824. Oates, J.F. and Anadu, P.A. 1982. Report on a survey of rainforest primates in southwest Nigeria. IUCN/SSC Primate Specialist Group Newsletter 2: 17.

825. Oliver, W.L.R. 1980. The Pigmy Hog: the biology and conservation of the Pigmy Hog Sus (Porcula) salvanius and the Hispid Hare Coprolagus hispidus. The Jersey Wildife Preservarion Trust, Special Scientific Report No.1.

826. Oliver, W.L.R. 1981. Pigmy Hog and Hispid Hare. Dodo 18: $10-20$.

827. Oliver, W.L.R. 1982. The coney and the yellow snake: the distribution and status of the Jamaican Hutia Geocapromys brownit and the Jamaican Boa Epicrates subflavis. Dodo 19: 6-33.

828. Oliver, W.L.R. 1985. The distribution and status of the Hispid Hare Caprolagus hispidus, with some additional notes on the Pygmy Hog Sus salvanius. Jersey Wildlife Preservation Trust, Jersey.

829. Oliver, W.L.R., Cox, C.R. and Dolar, L.L. 1991. The Philippine Spotted Deer conservation project. Onyx 25(4): 199-205. 
830. Oliver, W.L.R. and Santos, 1.B. 1991. Threotened endemic mammols of the Atlonric forest region of south-east Brozil. Jersey Wildlife Preservation Trust. Special Scientific Report No. 4.

831. Olivier, R.C.D. 1978. Distribution and status of the Asian Elephant. Oryx 14: 379-424.

832. Olrog, C.C. 1976. Sobre mamiferos del noroeste Argentino. Acta Zoologica Lilloana 32: 5-14.

833. Olrog, C.C. 1983. La situación presente de los carnívoros y ungulados Argentinos. I Reunión Iberoamericana de Zoólogos de Veriebrados, La Rabida, 1977, pp. 619-623.

834. Olrog, C.C and Lucero, M.M. 1981. Guía de los mamiferos Argenninas. Ministerio de Cultura y Educacion Fundacion Miguel Lillo, Tucuman.

835. Osborn, D.J. and Helmy, 1. 1980. The contemporary land mammals of Egypt (including Sinai). Fieldiana Zoology (N.S.) No.5. Public ation 1309

836. Osborne, B.C., Mallon, D.P. and Fraser, S.J.R. 1983. Ladakh, threatened stronghold of rare Himalayan mammals. Oryx 17(4): 182-189.

837. Osgood, W.H. 1932. Mammals of the Kelley-Roosevelts and Delacour A siatic expeditions. Field Musenm of Natural Histony, Zoological Series 18: 193-339.

838. Ovington, D. 1978. Australian endangered species: mammals, birds and repriles. Cassell Australia, Melbourne.

839. Pagès, E. 1970. Sur l'écologie et les adaptations de ['Oryctérope et des Pangolins sympatriques du Gabon. Biologia Gobonica 6(1): 27-92.

840. Pagès, E. 1975. Etude éco-éthologique de Monis tricuspis par radio-tracking. Mammolia 39(4): 613-641.

841. Palmeirim, J.M. and Hoffmann, R.S. 1983. Galemys pyrenaicus. Mammalian Species (American Society of Mammalogists) 207: 1-5.

842. Palmieri, R.P. 1983. Field investigations of Yak in the Nepal Himalaya. Narional Geographic Research Reporzs 12: 529-534.

843. Panwar, H.S. 1978. Decline and restoration success of the central lndian Barasingha Cervus duvauceli branderi. In: Anon, Threatened deer. Proceedings of a working meeting of the Deer Specialist Group of the SSC. IUCN, Morges, pp. 143-158.

844. Paradiso, J.L. and Nowak, R.M. 1972. Canis rufus. Mammalian Species (American Sociely of Mammalogists) 22.

845. Parker, 1. and Amin, M. 1983. Ivory crisis. Chatto and Windus, London.

846. Patterson, B.D. 1980. Eutamias quadrivittatus austrolis new subspecies Rodentia: Sciuridae from the Organ Mountains New Mexico, USA. Joumal of Mammalogy $61(3): 455-464$.

847. Patton, D. 1979. Sperm Whale stranding in Baja California. Terra, Los Angeles 17(3): 28-29.

848. Payne, J., Francis, C.M. and Phillipps, K. 1985. A field guide to the mammals of Bomeo. The Sabah Society/WWF Malaysia.

849. Payne, N. 1984. Fruit bat off the menu? Bots, newsletter of the 1UCN/SSC Chiroptera Specialist Group, (February).
850. Pearse, R.J. 1979. Distribution and conservation of the Australian Fur Seal in Tasmania. Victorian Naturalist 96(2): 48-53.

851. Pearson, O. 1951. Mammals in the highlands of southern Peru. Bullerin of the Museum of Comparative Zoology 106: 117-174.

852. Peck, J.M., Pelton, M.R., Picton, H.D., Schoen, J.W. and Zager, P. 1987. Grizzly Bear conservation and management: a review. Wildlife Saciety Bulletin 15(2) 160-169.

853. Peenen, P.F.D. van, Ryan, P. and Light, R. 1969. Preliminony idenificonion manual for mammals of South Vietnam. Smithsonian Institution, Washington.

854. Peres, C.A. 1989. A survey of a gallery forest primate community, Marajo Island, Pará, Brazil. Vida Sylvestra Neatrapical 2(1): 32-37.

855. Perez, M.C. 1983. Revisión de la sistemática de Gazello (Nanger) dama. I Reunión Iberoamericana de Zoólogos de Vertebrados, La Rabida, 1977, pp. 553-561.

856. Peris, S.J. 1981. Observations ornithologiques dans le sud ouest du Maroc. Bulletin de I'Institut Scientifique, Rabat 5: 135-141.

857. Perrin, W.F., Mitchell, E.D., Mead, J.G., Caldwell, D.K and Bree, P.J.H. van 1981. Stenella clymene, a rediscovered tropical dolphin of the Atlantic. Joumal of Mammology 62(3): 583-598.

858. Perry, J. and Horsemen, D.L. 1972. Caplive breeding of Orangutans. Zoologica Summer, 1972.

859. Petrides, G.A. 1965. Advisory report on wildlife and national parks in Nigeria 1962. Special publicatian No. 8. Americon Committee for Intemational Wildlife Protection.

860. Petter, J.-J. and Andriatsara fara, S. 1987. Les Lemuriéns de l'ouest de Madagascar. In: Anon, Priorités en marière de conservarion des espèces à Madagascar. IUCN, Gland.

861. Petter, J.-J. and Hladik, C.M. 1970. Observations sur le domaine vital et la densité de population de Loris tardigradus dans les forêts de Ceylan. Mammalia 3: 394 409 .

862. Petter, J.-J. and Petter-Rousseaux, A. 1979. Classification of the prosimians. In: Doyle, G.A. and Martin, R.D. (Eds), The sudy of prosimian behoviour. Academic Press, New York, pp. 1-44.

863. Petter, J.-J. and Petter-Rousseaux, A. 1960. Remarques sur la systématique du genre Lepilemur. Mammolio 24: 76-86.

864. Pfeffer, P. 1969. Considérations sur l'écologie des forêts claires du Cambodge oriental. Lo Terre et la Vie 23: 3-24.

865. Phillips, W.W.A. 1981. Manual of the mammals of Sri Lanka, par 2. Wildlife and Nature Protection Society of Sri Lanka.

866. Pikunov, D.G. and Korkishko, V.G. 1985. [The present distribution and numbers of Leopards (Panthera pardus) in the Soviet Far East]. Zoalagicheskii Zhumal 64: 897.905. (In Russian).

867. Pilleri, G. and Tagliavini, F. 1982. Observations on the ecology and distribution of the Susu (Platanista gangetica) in Nepalese rivers. Invesnigarians on Cetocea 13: 257-261.

868. Pinder, L. 1986. Studying the Maned Sloth. WWF Manthly Report November 1986: 295-296. 
869. Pine, R.H. 1973. Mammsls (exclusive of bats) of Belem, Para, Brazil. Acta Amazonica 3: 47-79.

870. Pine, R.H., Miller, F.D. and Schamberger, A.M.L. 1979. Contributions to the mammalogy of Chile. Mammalia 43: (3): 339-375.

871. Plotnekov, A.E., (Ed.) 1988. Red data book of Buryazskaya SSR. Knesnoy Publishers, Buryatskaya.

872. Poche, R.M. 1973. Niger's threatened park. Oryx 12(2): 216-222.

873. Poche, R.M. 1976. Notes on primates in Parc National du W du Niger, West Africa. Mammalia 40(2): 187-198.

874. Poduschka, W. 1979. Notes on the Giant Golden Mole Chrysospalar revelyani and its survival chances. Sonderdruck aus Zeischrifi für Säugetierkunde 45(4): 193 206.

875. Poduschka, W. 1982. The Giant Golden Mole. Oryx 16(3): 232-234.

876. Poduschka, W. and Richard, B. 1986. The Pyrenean Desman- an endangered insectivore. Oryx 20: 230-232.

877. Poirier, F.E. 1983. The Golden Monkey in the People's Republic of China. IUCN/SSC Primate Specialist Group Newsletier 3: 31-32.

878. Poirier, F.E. 1984. A status report on the Taiwan Macaque. IUCN/SSC Primate Specialist Group Newsletter 4: 44.

879. Poirier, F.E. and Davidson, D.M. 1979. A preliminary study of the Taiwan Macaque (Macaca cyclopis). Quarterly Joumal of the Taiwan Museum 32(3-4): 123-191.

880. Poirier, F.E. and Farslow, D. 1984. Status of the Crab-eating Macaque on Angaur Island, Palau, Micronesia. IUCN/SSC Primate Specialist Group Newsletter 4: 42-43.

881. Pollock, J. 1986. A note on the ecology and behaviour of Hapalemur griseus. Primate Conservation 7: 97-101

882. Povilitis, A. 1983 . The Huemul in Chile: national symbol in jeopardy? Oryx 17(1): 34-40.

883. Powell, J.A., Belitsky, D.W. and Rathbun, G.B. 1981. Status of the West Indian Manatee (Trichechus manatus) in Puerto Rico. Joumal of Mammalogy 62(3): 642-646.

884. Prater, S.H. 1971. The book of Indian animals. Bombay Natural History Society.

885. Prigioni, C., Bogliani, G. and Barbieri, F. 1986. The Otter Lutra lutra in Albania. Biological Conservation 36: 375 383.

886. Procter, J. 1963. A contribution to the natural history of the Spotted-necked Otter (Lutra maculicollis Lichtenstein) in Tanganyika. East African Wildlife Joumal 1: 93-102.

887. Prynn, D. 1980. Tigers and Leopards in Russia's far east. Oryx 15(5): 496-503.

888. Quinn, N.W.S. and Thompson.J.C. 1987. Dynamics of an exploited Canada Lynx population in Ontario. Joumal of Wildlife Management 51 (2): 297-305.

889. Rabinowitz, A. 1988. The Clouded Leopard in Taiwan. Oryx 22: 46-47.

890. Rabinowitz, A. 1989. The density and behaviour of large cats in a dry tropical forest mosaic in Huai Kha Khaeng Wildlife Sanctuary. Thailand. Natural History Bulletin of the Siam Saciety 37(2): 235-251.
891. Rabinowiz, A., Andau, P. and Chai, P.P.K. 1987. The Clouded Leopard in Malaysian Borneo. Oryx 21 : 107-111.

892. Rabor, D.S. 1968. Threatened species of small mammal: in tropical south east Asia: The problem in the Philippines. In: Talbot, L.M. and Talbot, M.H. (Eds), Conservation in Tropical Sauth East Asia. IUCN Publication (N.S.), No. 10, Gland, pp. 272-274.

893. Rahaman, H. and Parthasarathy, M.D. 1967. A population survey of the Bonnet Monkey (Macaca radiata Geoffroy) in Bangalore, South India. Journal of the Bombay Natural History Society 64: 251-255.

894. Rahm, U. 1955. Beobachtungen an den Schuppentieren: $M$. tricuspis und $\boldsymbol{M}$. longicaudata der Elfenbein Küste. Revue Suisse de Zoologie 62: 361-369.

895. Rahm, U. 1965. Distribution et écologie de quelques mammiferes de l'est du Congo. Zoologie Africaine 1(1): 149-166.

896. Rahm, U. 1966. Les mammiferes de la forêt équatoriale du l'est du Congo. Annales du Musée Royale de l'Afrique Centrale, Série in 8 vo's. Tervuren Sciences Zoologiques 149: 39-121.

897. Rahm, U. 1972. Zur vertreitung and okologie der säugetiere des Afrikanischen regenwaldes. Acta Tropica 29(4): $452-473$

898. Rahm, U. and Christiaensen, A.R. 1963. Les mammiferes de la région occidentale du Lac Kivu. Annales du Musée Royale de l'Afrique Centrale, Série in 8 vols. Tervuren Sciences Zoologiques 118: 1-83.

899. Rahm, U.H. 1970. Ecology, zoogeography and systematics of some African forest monkeys. In: Napier, J.R. and Napier, J.H. (Eds), Old World monkeys: evolution, systematics and behaviour. Academic Press, New York, pp. 591-626.

900. Rainier, Prince and Bourne, G.H. (Eds) 1977. Primate conservation. Academic Press, New York.

901. Rakotoarison, N., Mutschler, T. and Thalmann, U. 1993. Lemurs in Bemaraha (World Heritage Landsc ape, Western Madagascar). Onyx 27: 51-53.

902. Ralls, K. 1978. Tragelophus eurycerus. Mammalian Species (American Society of Mammalogists) 111: 1-4.

903. Ramachandran, K.K. 1989. Endangered Grizzled Giant Squirrel habitat. Joumal of the Bombay Naural History Sociery 86: 94-95.

904. Ramachandran, K.K. 1990. Recent evidence of the brown palm civet (sic). Paradocunus jerdoni, from Silent Valley National Park, India. Mustelid and Viverrid Conservation 3: 15 .

905. Ramirez. M. 1984. Ecology and conservation of the Moustached Tamarin in Peru. IUCN/SSC Primate Specialist Group Newsletter 4: 22-23.

906. Ramirez-P.J, López-W.R., Müdespacher, Z.C. and Lira, 1.E. 1983. Lista y hihliograpfia reciente de los mamiferos de Mexico. Universidad Autonoma Metropolitana, Ltapalapa.

907. Rand, D. 1983. Hamadryas Baboons in Yemen. IUCN/SSC Primate Specialist Group Newsletter 3: 26-27.

908. Ranjitsinh, M.K. 1978. The Manipur Brow-antlered Deer (Cervus eldi eldi). A case history. In: Anon. Threatened deer. Proceedings of a working meeting of the Deer Specialist Group of the SSC. IUCN, Morges. pp. 26-32. 
909. Rassi, P. and Vãisänen, R. 1987. Threatened animals and plants in Finland. English summary of the report of the Committee for the Conservation of Threatened Animals and Plants in Finland, Helsinki.

910. Rathbun, G.B. and Gache, M. 1980. Ecological survey of the Night Monkey, Aotus trivirgatus, in Formosa Province, Argentina. Primates 21(2): 211-219.

911. Rathbun, G.B., Powell, J.A. and Cruz, G. 1983. Status of the West Indian Manatee in Honduras. Biological Conservation 26(4): 301-308.

912. Rathore, F.S. 1990. Problems with Project Tiger, India. P. 8. In: Anon, Cat Specialist Group meeting reports. Cat News 12: 2-14.

913. Raven, H.C. 1935. Wallace's Line and the distribution of Indo-Australian mammals.

914. Raxworthy, C.J. 1986. The lemurs of Zahamena reserve. Primate Conservation 7: 46-48.

915. Redford, K.H. and Eisenberg, J.F. 1992. Mammols of the Neatropics. Vol.2. Chile, Argentina, Uruguay, Paraguay. University Chicago Press.

916. Reeves, R. 1982. What hope for North Allantic Right Whales? Oryx 16(3): 255-262.

917. Reeves, R., Mitchell, E., Mansfield, A. and McLaughlin, M. 1983. Distribution and migration of the Bowhead Whale, Boloena mysticetus, in the eastem north Arctic. Arctic 36(1): 5-64.

918. Reeves, R.R. 1978. Atlantic Wolrus fOdobenus rosmarus rosmanus): a literature survey and stotus repart. U.S. Department of Interior Wildlife Research Report no. 10.

919. Reeves, R.R. and Tracey, S. 1980. Monodon monoceros. Mammalian Species No. 127. pp. 1-7. American Society of Mammalogists.

920. Reich, L.M. 1981. Microtus pennsylvanicus. Mammalian Species No. 159: 1-8.

921. Reilly, S.B. 1981. Population assessment and population dynamics of the Califomia Gray Whale (Eschrichrius robustus). Dissertotion Abstracts Internationol, B. Science and Engineering 42(6): 2214.

922. Richardson, L. 1986. On the track of the last Black-footed Ferrets. Natural History 95(2): 69-77.

923. Ridgway, S., and Harrison, R. (Eds) 1981. Handbook of marine mammals, vol. 1 (Walrus, Seal Lions, Fur Seals, Sea Otter), pp. 1-235, and vol. 2. (True Seals), pp. 1-359. Academic Press, London.

924. Riecken, U. 1989. Status of the Oter (Lutra lutro) in Schleswig-Holstein (northern Germany). IUCN Otter Specialist Group Bulletin 4: 36.

925. Rieger, 1. 1981. Hyaena hyaena. Mammolian Species No. 150.

926. Rijksen, H.D. 1974. Orang-utan conservation and rehabilitation in Sumatra. Bialogical Conservation 6(1):2025.

927. Rijksen, H.D. 1978. A field study on Sumatran Orang Utans (Pongo pygmaeus abeli Lesson 1827), ecology, behoviour and conservation. Mededelingen Landbouwhogeschool, Wageningen.

928. Riney, T. 1964. Potential use of wildlife resources in Tunisian forest lands. FAO report to Government of Tunisia. Unpublished.
929. Roberts, M.S. and Gittleman, J.L. 1984. Ailurus fulgens. Mammolian Species No. 222, pp. 1-8. American Society of Mammalogists.

930. Roberts, T.J. 1977. The mammals of Pakistan. Benn, London.

931. Robineau, D. 1985. Données préliminaires sur la répartition du dauphin de Commerson Cephalorhynchus commersonii aux îles Kerguelen, en particulier dats le Golfe du Morbihan. Biological Conservation 31(1): 85-93.

932. Robineau, D. and Rose, J.M. 1982. Le Dugong, Dugong dugon (Muller 1776, Sirenia, Dugongidae) en République de Djibouti. Biological Conservation 24(3): 233-238.

933. Robinson, A.C., Robinson, J.F., Watts C.H.S. and Boverstock, P.R. 1976. The Shark Bay Mouse Pseudomys praeconis and other mammals on Bemier Island, Western Australia. Westem Australian Noturalist 13(7): 149-155.

934. Robinson, H.C. and Kloss, C.B. 1917. List of the mammals of Sumatra. Joumal of the Federated Malay States Museums 8(2): 73-80.

935. Robinson, P.T. 1970. The status of the Pygmy Hippopotamus and other wildlife in West Africa. M.Sc. thesis. Michigan State University, Michigan.

936. Robinson, P.T. 1971. Wildlife trends in Liberia and Sierra Leone. Oryx 11(2-3): 117-122.

937. Roche, J. 1971. Recherches mammalogiques en Guinea forestière. Bulletin du Muséum National d'Histoire Naturelle 16: 737-781.

938. Rodgers, W.A. and Homewood, K.M. 1982. Biological values and conservation prospects for the forests and primate populations of the Uzungwa Mountains, Tanzania. Biological Conservation 24(4): 285-304

939. Rookmaaker, L.C. 1983. Bibliography of the rhinoceros. A.A. Balkema, Rotterdam.

940. Rosevear, D.R. 1953. Checklist and atlas of Nigerian mammals. Government Printer, Lagos.

941. Rosevear, D.R. 1969. The rodents of West Africa. British Museum (Natural History), London.

942. Rosevear, D.R. 1974. The camivores of West Africa. British Museum (Natural History), London.

943. Roth, V. 1941. Notes and observations on animal life in British Guiana 1907-1941. A popular guide to colonial mammalia. Daily Chrotricle, Georgetown.

944. Roure, G. 1968. Petit atlas de classification, de morphologie, de repartition et de determination des animaux sauvages de Haute Volta et des pays voisins. Direction des eaux et forets. Ministere de l'Agriculture, Ouagadougon, Haute-Volta.

945. Rozhnov, V.V., Kuznetzov, G.V. and Pham Trong Anh. 1992. New distributional information on Owston's Palm Civel. Smoll Camivore Conservation 6: 7.

946. Rudran, R. and Eisenberg, J.F. 1982. Conservation and status of wild primates in Venezuela. Intermatianal Zoo Yearbook 22: 52-59.

947. Rylands, A. B. 1981. Preliminary field observations on the marmoset, Collithrix humeralifer intermedius (Hershkovitz, 1977 ) at Dardanelos, Rio Aripuana, Mato Grosso. Primates 22(1) 1981: 46-59.

948. Rylands, A.B. and Mittermeier, R.A. 1982. Conservation of primates in Brazilian Amazonia. Intermational Zoo Yearbook 22: 17-37. 
949. Sabados, K. and Simiak, M. 1981. [Distribution and management of the Brown Bear (Ursus arctos L.) in Slovakia]. Folia Venatoria 10-11: 15-33. (In Czech).

950. Sabater Pi, J. 1966. Los monos de Rio Muni y su distribucion. Zoo 6: 15-18.

951. Sabater Pi, J. 1981. Exploitation of Gorillas Gorilla gorilla gorilla Savage and Wyman 1847 in Rio Muni, Republic of Equatorial Guinea, West Africa. Biological Conservation 19(2): 131-140.

952. Sabater Pi, J. and Jones, C. 1967. Notes on the distribution and ecology of the higher primates of Rio Muni, West Africa. Tulane Srudies in Zoology 14: 101-109.

953. Saha, S.S. 1977. On some mammals recently collected in Bhutan. Joumal of the Bombay Natural History Society 74: $350-354$.

954. Saleh, M.A. 1987. The decline of gazelles in Egypt. Biological Conservarion 39: 83-95.

955. Salter, R.E. 1983. Summary of currently available information on internationally threatened wildlife species in Burma. FAO Field Document 7/83 FO: BUR/80/006

956. Salter, R.E. 1985. Conservation status of the Proboscis Monkey in Sarawak. Biological Conservarion 33: 119-132.

957. Salter, R.E. and Sayer, J.A. 1986. The Brow-antlered Deer in Burma - its distribution and status. Oryx 20: 241245.

958. Sanborn, C.C. 1929. The land mammals of Uruguay. Zoological series, Field Museum of Notural Histony Publication 265, 17(4): 147-165.

959. Sankaran, R. 1990. Status of the Swamp Deer Cervus duvauceli duvauceli in the Dudwa National Park, Uttar Pradesh. Joumal of Bombay Natural History Society 87 : 250-259.

960. Santiapillai, C. 1981 . On the ecology and conservation of the Dugong, Dugong dugon (Muller 1776) in Sri Lanka. Tigerpaper 8(1): 2-6.

961. Santiapillai, C. 1986. Planning a future for the Clouded Leopard. WWF Monthly Report September 1986: 233-238.

962. Santiapillai, C. 1990. Sumatran Tiger Panthera tigris sumatrae. Pp. 8-9. In: Anon, Cat Specialist Group meeting reports. Cat News 12: 2-14.

963. Santiapillai, C. 1992. Javan Rhinoceros in Vietnam. The Rhino Conservation Newsletter April 1992: 1-2.

964. Santiapillai, C. and Ashby, K.R. 1988. The Clouded Leopard in Sumatra. Oryx 22: 44-45.

965. Santiapillai, C. and Ramono, W.S. 1980. The status and conservation of the Malayan Tapir Tapirus indicus in Sumatrs, Indonesia. Tigerpaper 17(4): 6-11.

966. Santiapillai, C. Sukohadi, W. and Darmadja, B.P. 1990. Status of the Javan Rhino in Ujong Kulon National Park. Tigerpaper 17(2): 1-8.

967. Santiapillai, C. and Widodo Sukohadi Ramono 1985. On the status of the Tiger Panthera rigris sumatrae Pocock, 1829 in Sumatra. Tigerpaper 12(4): 23-29.

968. Santos, I.B., Oliver, W.L.R. and Rylands, A.B. 1987. Distribution and status of two species of tree porcupine, Chaetomys subspinosus and Sphiggurus insidiosus, in south-east Brazil. Dodo 24: 43-60.
969. Sarkar, H.B.D., Murali, S., Prasad, D.T., Shekarappa, B.M. and Vijayalakshmi, V. 1981. The population and distribution of the Slender Loris Loris tardigradus in Kamatska State. Tigerpaper 8(1): 7-10.

970. Sarker, S.U. and Sarker, N.J. 1984. Mammals of Bangladesh - their status, distribution and habitat. Tigerpaper 11(1): 8-13.

971. Sarro, A. and Oliveras, J.R.P. 1968. Expedicion a las islas del Banco de Arguin (Mauritiana). Andeola 12: 121141.

972. Sasaki, H. 1991. Sasaki, H. 1991. The present status of mustelids and viverrids in Japan. Mustelid and Viverrid Conservarion 4: 14-15.

973. Saunders, G.B., Holloway, A.D. and Handley, C.O. Jr. 1950. A fish and wildife survey of Guatemala. Special Scientific Report - Wildlife No. 5. United States Department of Interior, Washington, D.C.

974. Savidge, J.A. 1984. Guam: paradise lost for wildlife. Biological Conservation 30(4): 305-317.

975. Sayer, J. 1977. Conservation of large mammals in the Republic of Mali. Biological Conservation 12: 245-263.

976. Sayer, J. 1983. Lao People's Democratic Republic, nature conservation and national parks, final report. FAO.

977. Sayer, J. 1983. Nature conservation priorities in Loos. Tigerpaper 10(3): 10-14.

978. Sayer, J.A. and Green, A.A. 1984. The distribution and status of large mammals in Benin. Mammal Review 14(1): 37-50.

979. Schaaf, D. 1978. Some aspects of the ecology of the Swamp Deer or Barasingha (Cervus $d$. duvauceli) in Nepal. In: Anon, Threatened deer. Proceedings of a working meeting of the Deer Specialist Group of the SSC. IUCN, Morges, pp. 65-86.

980. Schad, R.C., Montgomery, G. and Chancellor, D. 1981. La distribucion y frecuencia del manati, en el lago Gatun, en el Canal de Panama. ConCiencia 8(2): 1-4.

981. Schaller, G. 1988. Snow Leopard in China. Cat News 9: 11.

982. Schaller, G., Ren Jurang and Qiu Mingiiang. 1988. Status of Snow Leopard Panthera uncia in Qinghai and Gansu provinces. Biological Conservation 45: 179-194.

983. Schaller, G.B. 1985. China's golden treasure. Intemarionai Wildtife 15(1): 29-31.

984. Schaller, G.B., Hu Jinchu Pan Wenshi and Zhu Jing 1985. The Giant Pandas of Wolong. University of Chicago Press, Chicago.

985. Schaller, G.B., Li Hong Talipu, Lu Hua, Ren Junrang, Qiu Mingjiang and Wang Haibin. 1987. Status of large mammals in the Taxkorgan Reserve, Xinjiang. China. Biological Conservarion 42: 53-71.

986. Schauenberg, P. 1969. Contribution à l'étude du Tapir pinchaque (Tapinus pinchaque) Roulin 1829. Revue Suisse de Zoologie 76(8): 211-256.

987. Scheffer, V.B. 1958. Seals, sea lions and walnuses, a review of the Pinnipedia. Oxford University Press, Oxford.

988. Schembri, P.J, and Sultana, J., (Eds) 1989. Red data baok for the Maltese lslands. Environment Division, Ministry of Education, Malta. 
989. Schober, W. 1984. The lives of bats. Croom Helm, London.

990. Schomber, H.-W. and Kock, D. 1960. The wildlife of Tunisis, part 2: some larger mammals. African Wildife 14(4): $277-282$.

991. Schouteden, H. 1948. Faune du Congo Belge et de Ruanda-Urundi, 1 - mammiferes. Annales du Musée Royale du Cango Belge, Série in 8 vals, Tervuren Sciences Zoalagiques 1: 1-331.

992. Schreiber, A., Wirth, R., Riffel, M. and van Rompaey, H. 1989. Weasels, civets, mongoases, and their relarives. An action plan for the conservation of mustelids and viverrids. IUCN, Gland.

993. Seebeck, J.H. and Johnston, P.G. 1980. Patoraus longipes (Marsupialia: Macropodidae); a new species from eastem Victoria. Australian Joumal of Zoology 28: 119-134.

994. Seidensticker, J., Eisenberg, J.F. and Simons, R. 1984. The Tangjiahe, Wanglang, and Fengtongzhai Giant Panda reserves and biological conservation in the People's Republic of China. Biological Conservation 28(3): 217-251.

995. Sergeant, D., Ronald, R., Boulva, J. and Berkes, F. 1979 The recent status of Manachus monachus the Mediterranean Monk Seal. In: Ronald, K. and Duguy, R. (Eda), The Mediterranean Mank Seal. UNEP Technical Series, volume 1. Pergamon Press, Oxford, pp. 31-54.

996. Setzer, H.W. 1956. Mammals of the Anglo-Egyptian Sudan. Proceedings of the U.S. Natianal Museum 106: 447-615.

997. Shahi, S.P. 1982. Status of the Grey Wolf (Canis lupis pallipes Sykes) in India - a preliminary survey. Joumal of the Bombay Natural History Saciery 79(3): 493-502.

998. Shahi, S.P. and Chowdhury, S. 1986. Elephants in central India. WWF Manthly Report January 1986: 19-22.

999. Shellhammer, H. 1982. Reithrodantomys raviventris. Mammalian Species (American Society of Mammalogists) 169: 1-3.

1000. Sheng He-lin and Lu Ho-gee 1980. Current studies on the rare Chinese Black Muntjac. Joumal of Natural Histary 14(6): 803-807.

1001. Sheppe, W. and Haas, P. 1976. Large mammal populations of the lower Chobe, Botswana. Mammalia 40(2): 223-243.

1002. Sholley, C. 1989. Being friends. BBC Wildlife 7(10): 642 646.

1003. Shoshani, J. and Eisenberg, J.F. 1982. Elephas maximus. Mommalian Species (American Society of Mammalogists) 182: 1-8.

1004. Sidney, J. 1965. The past and present distribution of some African ungulates. Transacrions of the Zoalogical Sociery of London 30: 1-397.

1005. Sidorovich, V.E. 1992. Comparative analysis of the diets of European Mink (Mustela lutreola), American Mink (M. vison), and Polecat (M. putorius) in Byelorussia.

1006. Silkilwssha, F. 1981. The distribution and conservation status of the Zanzibar Red Colohus. African Joumal of Ecalogy 19: 187-194.

1007. Simonetta, A. 1984. On the status of antelopes in Somalia. IUCN/SSC Gnusletter May: 7-8.
1008. Simons, E.L. 1988. A new species of Propithecus (Primates) from north-east Madagascar. Falia primatologica 50: 143-151.

1009. Simons, H. 1986. Preliminary report of the survey of the Gunung Niut Nature Reserve, West Kalimantan. Unpublished.

1010. Singh, Arjan, 1978. The status of the Swamp Deer (Cervus $d$. duvauceli) in the Dudhwa National Park. In: Anon, Threatened deer. Proceedings of a working meeting of the Deer Specialiat Group of the SSC. IUCN, Morges, pp. 132-142.

1011. Singh, Ashbindu 1980. World's most endangered mammal - Manipur Brow-antlered Deer (Cervus eldi eldi). Tigerpaper 7(2): 26-27.

1012. Skinner, C. 1985. Report on a field study of Geoffroy's Tamarin in Panama. Primate Conservation 5: 22-24.

1013. Skinner, J.D., Fairall, N. and Bothma, J.du P. 1977. South A frican red data book - large mammals. Cooperative Scientific Programmes Council for Scientific and Industrial Research. South African National Scientific Programme Report No. 18.

1014. Skinner, J.D. and Smithers, R.H.N. 1990. The mammals of the Southem African subregian. University of Pretoria, Pretoria.

1015. Smet, $K$, de 1984. On the status of antelopes in Algeria. IUCN/SSC Gnusletter May: 7-8.

1016. Smit, C.J. and van Wijngaarden, A. 1976. Threatened mammals in Eurape. Nature and Environment Series No. 10. European Committee for the Conservation of Nature and Natural Resources, Strasbourg.

1017. Smithers, R.H.N. and Tello, J.L.P. Lobao 1976. Checklist and atlas of the mammals of Moçambique. Museum Memair, The Trustees of the National Museum of Rhodesia 8: 1-184.

1018. Smithers, R.H.N. 1966. The mammals of Rhodesia, Zambia and Malawi. Collins, London.

1019. Smithers, R.H.N. 1971. The mammals of Botswana Museum Memoir, The Trustees of the National Museum of Rhodesia 4: 1-340.

1020. Smithers, R.H.N. 1983. The mammals of the Southem African subregion. University of Pretoria, Pretoris.

1021. Soini, P. 1982. Ecology and population dynamics of the Pygmy Marmoset, Cebuella pygmaea.Falia Primatologica 39(1-2): 1-21.

1022. Soini, P. 1982. Primate conservation in Peruvian Amazonia Intemational Zoo Yearbaok 22: 37-47.

1023. Soini, P. 1986. A synecological study of a primate community in the Pacaya-Samiria National Reserve, Peru. Primate Conservarion 7: 63-71.

1024. Sokolov, V.E. and Orlov, V.N. 1980. Opredelitel' mlekopilayushchikh Mongol'skoi Narodnai Respubliki. Nauka, Moscow.

1025. Sokolov, V.G. et al. 1986. [Taxonomic list of the mammals of Vietnam]. In: Sokolov, V.E. (Ed.), [Mammais and birds of Vietnam and their ecology]. Nauka, Moscow. (In Russian).

1026. Southwick, C. and Siddiqi, M.F. 1983. Status and conservation of Rhesus Monkeys in India. In: Seth, P.K. (Ed.), Perspecnives on primate bialogy, Today and Tomorrow's Publisher, New Dehli, pp. 227-236. 
1027. Southwick, C. and Siddiqi, M.F. 1985. The Rhesus Monkey's fall from grace. Natural History 94(2): 63-70.

1028. Southwick, C. and Southwick, K.L. 1985. Rhesus Monkeys in Burma. Primate Conservatian 5: 35-36.

1029. Sowls, L.K. 1984. The Peccaries. University of Arizona Press, Arizona.

1030. Spinage, C.A. 1980. Gorillas in the Central African Republic. Unpublished.

1031. Spinage, C.A. 1980. Parks and reserves in Congo Brazzaville. Oryx 15(3): 292-295.

1032. Spinage, C.A. 1985. The elephants of Burkina-Faso, West A frica. Pachyderm 5: 2-5.

1033. Spinage, C.A. 1990. Botswana's problem elephanta. Pachyderm 13: 14-19.

1034. Sprague, D.S. 1986. Conservation of the monkeys and forests of Yakushima, Japan. Primate Canservation 7: 5557.

1035. Stallings, J.R. and Mittermeier, R.A. 1983. The Black-tailed Marmoset (Callithrix argentata melanura) reconded from Paraguay. American Jaumal of Primatology 4(2): 159-163.

1036. Stander, P. 1990. Lions Panthera lea in Etosha National Park, Namibia. Pp. 5-7. In: Anon, Cat Specialist Group meeting reports. Cat News 12: 2-14.

1037. Starck, D. 1974. Die sāugetiere Madagaskars, ihre lebenstraume und ihre geschichte. Sitzungsherichte der Wissenschafilichen Gesellschaft an der Joharn Wolfgang Gaethe - Universitat Frankfuri/Main 11(3): 67-124.

1038. Starin, E.D. 1981. Monkey moves. Natural Histary 90(9): 37-43.

1039. Stsrin, E.D. 1989. Threats to the monkeys of the Gambia. Oryx 23: 208-214

1040. Stebbings, R.E. 1984. A draft cammunity list of threatened species af wild fora and vertebrate fauna. Pan $V$ bats: species reparts. Bat Group, Species Survival Commission, IUCN/Institute of Terrestrial Ecology, NERC.

1041. Stenman, O. 1978. Legislation, protection and research on seals in Finland. Riistatieteellisiä Julkaisuja 37: 8.

1042. Stone, D. 1991. Man and Desman. BBC Wildife 9(8): 538541 .

1043. Stone, G.S., Katona, S.K. and Tucker, E.B. 1987. History, migration and present status of Humpback Whales Megaptera novaeangliae at Bermuda. Bialagical Conservatian 42: 133-145.

1044. Strahan, R. 1983. Complete book of Australian mammals. Angus and Robertson, Sydney.

1045. Strandgaand, H. and Asferg, T. 1980. The Danish game bag record II. Danish Review of Game Bialagy 11: 1-112.

1046. Strange, 1.J. 1992. A field guide to the wildlife of the Falkland Islands and South Geargia. Harper Collins Publishers, London.

1047. Struhsaker, T.T. 1971. Notes on Cercocebus $a$, anys in Senegal, West Africa. Mammalia 35: 343-344.

1048. Struhsaker, T.T. 1973. A recensus of Vervet Monkeys in the Masai-Amboseli Game Reserve, Kenya. Ecalagy 54(4): 930-932.

1049. Struhsaker, T.T. 1975. The Red Colobus Mankey. Chicago University Press, Chicago.
1050. Struhsaker, T.T. 1981. Forest and primate conservation in East Africa. African Jaurnal of Ecology 19: 99-114.

1051. Stuart, C.T. 1980. The distribution and status of Man's remmincki Smutt 1832. (Pholidota: Manidae). Saugetierkundliche Mitzeilungen 28: 123-129.

1052. Stuart, C.T. 1985. The status of two endangered carnivores occurring in the Cape Province, South Africa, Felis serval and Lutra maculicallis. Bialagical Conservarion 32(4): 375-382.

1053. Stuan, C.T., Macdonald, I.A.W. and Mills, M.G.L. 1985. History, current atatus and conservation of large mammalian predators in Cape Province, Republic of South Africa. Bialogical Canservation 31(1): 7-19.

1054. Stubbe, M. 1979. Der Fischotter Lutra lutra (L., 1758) in den Nondbezinken der DDR. Archiv der Freunde der Naturgeschichie in Mecklenburg 17: 61-93.

1055. Sugiyami, Y. and Parthasarathy, M.D. 1978. Population change of the Hanuman (Presbytis entellus), 1961-1976, in Dharwar Area, India. Jaumal af the Bambay Naural History Society 75: 867-867.

1056. Sukumar, R. 1986. Elephants in south India. WWF Manthly Repan January 1986: 23-32

1057. Sun Chon-shuo and Gao Yao-ting 1976. [A new record of Chinese felid from Yunnan - Pardafelis marmarata]. Acta Zoalagica Sinica 22(3): 304 (In Chinese).

1058. Susman, R.L. and Kabongo, K.M. 1984. Update on the Pygmy Chimp in Zaire. IUCN/SSC Primate Specialist Group Newsletter 4: 34-36.

1059. Sussman, R.W. 1975. A preliminary study of the behavior and ecology of Lemur fulvus rufus. Pp. 237-257. In: Tattersall 1. and R.W. Sussman (Eds), Lemur biolagy, Plenum Press, New York.

1060. Sussman, R.W. 1977. Feeding behaviour of Lemur catta and Lemur fulvus. Pp. 1-37. In: Clutton-Brock, T. (Ed), Primate ecalogy: sudies of feeding and ranging behaviaur in lemurs, mankeys and apes. Academic Press, London.

1061. Sussman, R.W. and Richard, A.F. 1986. Lemur conservation in Madagascar: the status of lemurs in the south. Primate Conservation 7: 86-92.

1062. Sussman, R.W., Richard, A.F. and Rakotomanga, P. 1987. La conservation des Lémuriens à Madagascar: leur statu dans la sud. In: Anon, 1987. Priorités en matière de canservation des espèces à Madagascar. IUCN, Gland.

1063. Sussman, R.W. and Tattersall, 1. 1980. A preliminary study of the Crab-eating Macaque (Macaca fascicularis) in Mauritius. Mauritius Instiune Bulletin 9(1): 31-51.

1064. Sussman, R.W. and Tattersall, I. 1986. Distribution, abundance, and putstive ecological strategy of Macaca fascicularis on the island of Mauritius, southwestern Indian Ocean. Falia Primatolagica 46(1): 28-43.

1065. Swank, W.G. and Teer, J.G. 1989. Status of the Jaguar 1987. Oryx 23: 14-21.

1066. Sweeney, R.C.H. 1959. A preliminary annotored checklist af the mammals af Nyasaland. Nyasaland Society, Blantyre.

1067. Swynnerton, G.H. and Hayman, R.W. 1951. A checklist of the land mammals of the Tanganyika Territory and the Zanzibar Protectorate. Jaumal of the East African Natural Histary Saciery 20: 274-392. 
1068. Sytnik, K.M, Grodzirtsky, A.M., Topachevsky, V.A., Chopik, V.1. and Fedorenko, A.P. (Eds) 1979. Red Data Book of the Ukrainian SSR. Naukova Dumka Publishers, Kiev.

1069. Taber, R. 1974. Endangered wildlife of Chile. Pp. 247251. In: Anon, World Wildlife yearbook 1973-4. WWF, Gland.

1070. Tahiri-Zagrët, C. 1976. Le Cercopithecidac de Côte d'Ivoire. Bulletin de I'Institut Fondomental de l'Afrique Noire 38(1): 206-230.

1071. Tallec, J. le 1979. Le grande faune du Sénégal: les mammiferes. Les Nouvelles Editions Africaines.

1072. Tan Beng-jie 1985. The status of primates in China. Primate Conservation 5: 63-81.

1073. Tan Bang-jie 1989. Conservation and economic importance of the mustelids and viverrids in China. Mustelid and Viverrid Conservation 1: 5-6.

1074. Tappen, N.C. 1960. Problems of distribution and adaptation of the African monkeys. Current Anthropology I: $91-120$.

1075. Tappen, N.C. 1964. Primate studies in Sierra Leone. Current Anthropology 5(4): 339-340.

1076. Tate, G.H.H. 1931. Random observations on habits of South American mammals. Joumal of Mammalogy 12: 245-256.

1077. Tattersall, 1. 1982. The primates of Madagascar. Columbia University Press, Columbia

1078. Tattersall, I. 1983. Studies of the Comoro lemurs: a reappraisal.IUCN/SSC Primate Specialist Group Newsletter 3: 24-26.

1079. Tattersall, I. 1986. Notes on the distribution and taxonomic status of some subspecies of Propithecus in Madagascar. Folio Primatologica 46(1): 51-63

1080. Taub, D.M. 1975. A report on the distribution of the Barbary Macaque in Morocco and Algeria. Unpublished

1081. Taylor, M.E. 1986. The biology of the Four-toed Mongoose Bdeogale crassicauda. Cimbebasia 8: 187-193.

1082. Taylor, M.E. 1987. Bdeogale crassicaudo. Mammalian Species (American Society of Mammalogists) 294.

1083. Taylor, R.H. 1982. New Zealand Fur Seals at the Bounty Islands. New Zeolond Journal of Marine and Freshwoter Research 16(1): 1-9.

1084. Teleki, G. 1980. Hunting and trapping wildlife in Sierrs Leone: aspects of exploitation and exporation. Unpublished.

1085. Teleki, G. 1980. Status of the hippopotamus Choeropsis liberiensis in Sierra Leone: a preliminary report on distribution. Unpublished.

1086. Teleki, G. 1980. Status report on wildlife survey project, Sierra Leone, West Africa, conducted Novemher 1979 through May 1980. Unpublished.

1087. Teleki, G. 1989. Population status of wild Chimpanzees (Pan troglodytes), and threats to their survival. In: Heltne. P.G. and L.A. Marquadt (Eds), Understanding Chimpanzees. Harvard University Press, Cambridge.
1088. Teleki, G. and Baldwin, L. 1979. Known and estimated distributions of extant chimpanzee populations (Pan troglodytes and Pan paniscus) in Equatorial Africa. Special report for IUCN/SSC Primate Specialist Group, June 1979. Unpublished.

1089. Tello, J. 1988. Cats in Mozambique. Cat News 8: 16-18.

1090. Tenaza, R. 1989. Primates on a precarious limb. Primate Conservation 1989: 27-36.

1091. Thalen, C.P. 1975. The Caracal Lynx (Caracal caracal schmitzi) in Iraq: earlier and new records, habitat and distribution. Bulletin of the Natural History Research Center University of Baghdad 6(1): 1-20.

1092. The Latvian SSR Acadamy of Sciences Institute of Biology. 1985. Red dala book of the Latvian SSR. Rare and endangered species of animals and plants. Zinatne Publishing House, Riga.

1093. Thomas, O. 1904. On mammals from the Island of Fernando Po, collected by Mr E. Seimund. Proceedings of the Zoological Society of London: 183-193.

1094. Thompson, C.O. 1982. Myotis sodalis. Mammalian Species (American Society of Mammalogists) 163.

1095. Thorington, R.W. 1988. Taxonomic status of Saguinus iripartitus. American Joumal of Primatology 15: 367-371.

1096. Thornback, L.J. 1981. Black Rhino. In: Thornback, J., IUCN red data book: mammalia, IUCN Conservation Monitoring Centre, Cambridge.

1097. Thornhack, L.J. 1983. Wild cattle, bison and buffaloes, their stalus and polential value. IUCN Conservation Monitoring Centre, Cambridge.

1098. Thornback, L.J. and Jenkins, M. 1982. The IUCN mammal red data book, port 1. IUCN, Gland.

1099. Thoules, C. 1987. Kampuchean Wildlife - survival agairıst the odds. Oryx 21: 223-228.

1100. Thouless, C. 1991. A report of the Laikipia elephan count, 1990. Pachyderm 14: 32-26.

1101. Thouless, C.R., Grainger, J.G., Shobrak, M. and Habibi, K. 1991. Conservation status of gazelles in Saudi Arabia. Bialogical Conservation 58(1): 85-98.

1102. Thys van den Audenaerde, D.F.E. 1977. Description of a new monkey skin from East Ceniral Zaire is probably a new monkey species. (Mammalia, Cercopithecidae). Revue Zoologique Africaine 91 : 1000-1010.

1103. Torres, D. and Aguayo, A. 1984. Presence of Arctocephalus tropicalis (Gray 1872) at the Juan Fernandez Archipelago, Chile. Acra Zoologica Fennica 172: $133-134$

1104. Trotignon, J. 1975. Le status et la conservation de l'addax et de l'oryx et de la faune associée en Mauritanie. Report to JUCN, Morges. Unpublished.

1105. Tumlison, R. 1987. Felis lynx. Mammalian Species (American Society of Mammalogists) 269: 1-8

1106. Turan, N. 1984. Türkjye'nin av ve yaban hayvanlar memetiler. Ongun Kardesler Matbaacilik Sanayii, Ankara.

1107. Tutin, C.E.G. and Fernandez, M. 1984. Nationwide census of Gorilla (Gorilla g . gorilla) and Chimpanzee (Pan troglodyves) populations in Gabon. American Joumal of Primatology 6: 313-336.

1108. Tyler, S. 1975. The Simien Fox. Wildlife 17(12): 565. 
1109. U Tun Yin 1967. Wild animals of Burma. Rangoon Gazette, Rangoon.

1110. U Tun Yin 1970. The Dugong, Dugong dugon (Müller) in Burmese watera. Journal of the Bambay Natural Histary Saciety 67: 326-327.

1111. U.S. Fish and Wildlife Service. 1987. Recovery plan for the Alabama Beach Mouse (Peromyscus polionotus ammobates), Perdido Key Beach Mouse (P.p.trissyllepsis), and Choctawhatchee Beach Mouse (P.p.allophrys). U.S. Fish and Wildlife Service, Allanta, Georgia.

1112. Uthai Treesucon 1983. Agile Gibbons in Thailand endangered. Conservation News 5: 30-32.

1113. Valverde, J.A. 1957. Aves del Sahara Español. Instituto de Estudios Africanos, Consejo Superior de Investigaciones Cientificas, Madrid.

1114. van Bemmel, A.C.V. 1949. Notes on Indo-Australian mammals. Treubia 20: 375-377.

1115. van Bree, P.J.H. 1968. Über ein Examplar von Lutrogale perspicillata (Geoffroy, 1826) (Mammalia, Camivora) aus Bhutan. Zool. Anz. 180: 162-164.

1116. van Bree, P.J.H. and Khan, M.K. hin M. 1992. On a Fishing Cat from continental Malaysia. Zeitschrift für Säugetierkunde 57: 179-180.

1117. van den Brink, F.H. 1967. A field guide to the mammals of Britain and Europe. Collins, London.

1118. van der Zon, A.P.M. 1979. Mammals of Indonesia. UNDP/FAO National Park Development Project, Bogor. FO/INS/78/061 Special Report.

1119. Varona, L.S. 1974. Catalogo de los mamiferos vivientes y extinguidos de las Antillas. Academia de Ciencias de Cuha.

1120. Varona, L.S. 1980. Mamiferns de Cuba. Genta Nueva, Havana.

1121. Varona, L.S. 1986. Taxones del subgénero Mysateles en Isla de la Juventud, Cuba. Descripción de una nueva especies. Poeyana 315: 1-2.

1122. Vaughan, C. 1983. A report on dense forest habitat for endangered wildlife species in Costa Rica. Environmental Sciences School, National University, Heredia, Costa Rica. Unpublished.

1123. Vaz-Ferreira, R. 1983. Aspectos eto-ecológicas, explotación de algunos Otariidos. I Reunión Iberoamericana de Zoólogos de Vertehrados, La Rabida, 1977, pp. 263-282

1124. Venegas, C.C. and Sielfeld, K.W. 1978. Registros de Mesoplodon layardii y otros cetaceos en Magallanes. Anales del Instiuto de la Patagonia 9: 171-177.

1125. Verheyen, W.N. 1963. New data on the geographical distribution of Cercopithecus (Allenopitheciss) nigroviridis Pocock, 1907. Revue de Zoologie et de Botanique Africaines 68(3-4): 393-396.

1126. Verschuren, J. 1965. Contrihution à l'ecologie des grands mammiferes. Exploration du National Parc de la Kagera. Inst. Parcs Nat. Congo-Rwanda (2) 1: 1-66.

1127. Verschuren, J. 1978. Burundi and wildlife. Problems $n f$ an overcrowded country. Oryx 14(3): 237-240.

1128. Verschuren, J. 1978. Les gonads mammiferes du Burundi. Mammalia 42: 209-224.

1129. Verschuren, J. 1980. Saving Paraguay's wilderness. Onyx 15(5): $465-470$.
1130. Verschuren, J. 1983. Conservation of tropical rain forest in Liberia. IUCN, Gland.

1131. Verschuren, J. Heymans, S.C. and Delvingt, W. 1989. Conservation in Benin - with the help of the European Economic Community. Oryx 23: 22-26.

1132. Vidic, J. (Ed.) 1992. Varstvo narave 17. Revija za teorijo in Prakso Varstua Naravne Dediščine. Ljubljana, Slovenia.

1133. Villa, B.R. and Colomero, L.C. 1982. Distribucion y presencia del manati o talcamichin (Trichechus manatus) en México. Ann. Inst. Biol. Univ. Ausonama México. 51(1): 703-708.

1134. Vincent, F. 1969. Contribution à l'étude des prosimiens Africains: Galago de Demidoff, reproduction (biologie, anatomie, physiologie) et comportement. Vols. 1 and 2. Ph. D. dissertation, Faculty of Sciences, Paris.

1135. Vincent, F. 1972. Prosimiens Africains: V. Répartition géographique de Euoticus inustus. Annales de la Faculté des Sciences de l'Université de Cameroun 10: 135-141.

1136. Vivien, 1. 1991. Faune du Cameroun. Guide des mammiferes at poissuns. GICAM et Ministère de la Coopération et du Développement, Cameroun.

1137. Vogel, P. et al. 1986. A contrihution to the taxonomy and ecology of shrews from Crete and Turkey. Acta Theriologica 31: 537-545.

1138. von Genov, P., Giovanna Massei, S. and Gànĉev, R.S.Z. 1990. Verbreitung und zahlenmäßige Stärke der Gemse (Rupicapra nupicapra balcanica Bolkay, 1925) in Bulgarien. Zeitschrift fiur Jagdwissenschaft 36: 1-8.

1139. Voskar, J. 1983. Present problems of Wolf preservation in Czechoslovakia . Acta Zoologica Fennica 174: 287-288.

1140. Wabakken, P., Sorensen, O.J. and Kvam, T. 1983. Wolves (Canis lupus) in southeastem Norway. Acla Zoologica Fennica 174: 277.

1141. Walker, P. 1974. History of three little-known New World populations of mac aques. Primate Newsletter 13(1): 16-18.

1142. Walther, F.R., Mungall, E.C. and Grau G.A. 1983. Gazelles and their relatives. A study in territorial behaviour. Noyes Puhlications, New Jersey.

1143. Walton, D.W.. (Ed.) 1988. Zoological catalogue of Australia. Volume 5. Mammalia. Australian Government Publishing Service, Canberra.

1144. Wang Q., Hu X. and Yan Y. 1982. On a new subspecies of Siberian Musk-deer, Moschus moschiferus anhuiensis subsp. nov.. from Anhui, China. Acta Theriologica Sinica 2(2): 133-138.

1145. Wang Sung 1990. Cat problems in China. P. 10. In: Anon, Cat Specialist Group meeting reports. Cat News 12: 2-14.

1146. Wang Xiaoyan. 1987. Conservation of precious and rare aquatic animals in China. Paper presented at International Conference on Wildife Conservation in China J987. 14-19 July 1987, Beijing

1147. Watson, L. 1981. Sea guide to whales of the world. Hutchinson, London.

1148. Watts, C.H.S. 1979. The status of endangered Australian rodents. In: Tyler. M.J. (Ed.), The staus of endangered Australasian wildlife. Royal Zoological Society of South Australia, pp. 75-83.

1149. Watts, C.H.S. and Aslin, H.J. 1981. The rodents of Australia. Angus and Rohertsnn, Sydney. 
1150. Watts, E.S., Rico-Gray, V. and Chan, C. 1986. Monkeys of the Yucatán Peninsula, Mexico: preliminary survey of their distribution and status. Primate Conservation 7: 17 22.

1151. Wayre, P. 1978. The status of otters in Malaysia, Sri Lanka and ltaly. In: Duplaix, N. (Ed.), Otters. Proceedings of the first working meeting of the IUCN/SSC Otter Specialist Group, Paramaribo, Suriname 27-29 March 1977. IUCN, Morges, pp. 152-155.

1152. Weber, A.W. and Vedder, A. 1983. Population dynamics of the Virunga Gorillas: 1959-1978. Biological Conservation 26(4): 341-366.

1153. Weber, B. 1979. Gorilla problems in Rwanda. Swara 2(4): 29-32.

1154. Weinberg, D. 1986. Decline and fall of the Black-footed Ferret. Natural History 95(2): 63-69

1155. Weisbein, Y. and Mendelssohn, H. 1990. The biology and ecology of the Caracal Felis caracal in the northern Aravah. Cat News 12: 20-22

1156. Wemmer, C. and Watling, D. 1986. Ecology and status of the Sulawesi Palm Civel Macrogalidia musschenbraekii Schlegel. Biological Conservotion 35: 1-17.

1157. Westem, D. and Vigne, L. 1985. The status of thinos in Africa. Swara 8(2): 10-12.

1158. Westing, A.H. and Westing, C.E. 1981. Endangered species and habitats of Viet Nam. Environmentol Conservotion 8(1): 59-62.

1159. Wetzel, R.M. 1975. The species of Tamandua Gray Proceedings of the Bialogical Society of Washington 88: 95-112.

1160. Wetzel, R.M. 1980. Revision of the naked-tailed armadillos, genus Cobassous. Annals of the Camegie Museum 49: 323-357.

1161. Wetzel, R.M. 1982. Systematics, distribution, ecology and conservation of South American edentates. In: Maros, M.A. and Genoways, H.H. (Eds), Mammalian biology in South America. Pymatuning Laboratory of Ecology, University of Pittsburgh, Special Publication, Pittsburgh, pp. 345-375.

1162. Wetzel, R.M. and Lovett, J.W. 1974. A collection of mammals from the Chaco of Paraguay. The University of Connecricut Occasional Popers 2(13): 203-216.

1163. Wetzel, R.M. and Mondolfi, E. 1979. The subgenera and species of long-nosed armadillos, genus Dasypus L. In: Eisenberg, J.F. (Ed.), Vertebrate ecalogy in the northem Neotropics. Smithsonian, Washington, D.C.

1164. Wheeler, M.E. 1980. The status of the Marianas Fruit Bat on Saipan, Tinian, and Rots. Elepaio 40(8): 109-113.

1165. Wheeler, M.E. and Aguon, C.F. 1978. The current status and distribution of the Marianas Fruit Bat on Gusm. Aquatic and Wildlife Resources Division Technical Reporn, No. 1. Department of Agriculture, Guam.

1166. Whitehead, G.K. 1972. Deer of the world. Constable, London.

1167. Whitten, A.J. 1982. Home range use by Kloss Gibbons (Hylobotes klossii) on Siberut 1sland, Indonesia. Animal Behaviour 30: 182-198.
1168. Whitten, A.J. and Whitten, J.E.J. 1982. Preliminary observations of the Mentswai Macaque on Siberut Island, Indoneaia. Intemational Joumal of Primatology 3(4): 445-459.

1169. Wildlife Department, Guangdong Institute of Entomology, Department of Biology, Sun Yetsen University 1983. [The birds and mammals of Hainan]. Science Press. (In Chinese).

1170. Wiles, G.J., Lemke, T.D. and Payne, N.H. 1989. Population estimates of fruit bats (Pleropus mariannus) in the Mariana Islanda. Conservation Biology 3(1): 66-76.

1171. Wilkinson, A.F. 1974. Areas to preserve in Sierra Leone. Oryx 12(5): 596-597.

1172. Willan, R.G.M. 1973. Tunisia's wildlife. Oryx 12(1): 74-76.

1173. Willan, R.G.M. 1977. Consultation d'experts sur les parcs marins et les zones humides de la région Méditerranéene. Tunis 12-14 January 1977. Visite au futur Parc National de l'lchkeul.

1174. Williams, N.L. 1985. Black Rhino in South Luangwa National Park: their distribution and future protection. Oryx 19(1): 27-33

1175. Wilson, R.T. 1980. Wildlife in northem Darfur, Sudan: a review of its distribution and status in the recent past and at present. Bialogicol Conservation 17: 85-101.

1176. Wilson, V. 1988. Cheetah in Zimbabwe. Cat News 8:910.

1177. Wilson, V.J. 1975. Mammals of the Wankie National Park, Rhodesia. Museum Memoir, Trustees of National Museums and Monuments of Rhodesia 5: 1-147.

1178. Wilson, V.J. 1985. Visit to Côte d'lvoire, West Africa, September-October 1985. Chipangali Wildife Trust, Report No.1.

1179. Wilson, V.J. 1990. Preliminary survey of the duikers and other large mammals of Burundi, East Africo. Chipangali Wildlife Trust, Bulawayo, Zimbabwe.

1180. Wilson, V.J. and Wilson, B.L.P. 1991. Notes on the duikers of Sierra Leone. Nature et Foune 7: 24-35.

1181. Winter, J.W. 1979. The status of endangered Australian Phalangeridae, Petauridae, Burramyidae, Tarsipedidae and the Koala. In: Tyler, M.J. (Ed.), The status of endangered Australasian wildlife. Royal Zoological Society of South Australia, pp. 45-59.

1182. Wirth, R. 1991. Die vergessenen Riesen hömchen. Zoologische Gesellschaft Straund Populationsschutz 7(1): ]-2.

1183. Woltheim, J.H. 1983. Primates of the world, distribution, consenvation and abundance. University of Washington Press, Seattle.

1184. Won Pyong-Oh 1976. Checklist of the mammals of Korea. Institute of Omithology, Kyung Hee Univeraity, Seoul.

1185. Woods, C.A. 1981. Last endemic mammals in Hispaniola. Oryx 16(2): 146-152.

1186. Woods, C.A., Post, W. and Kilpatrick, C.W. (1982). Microtus pennsylvanicus (Rodentia: Muridae) in Florida: a Pleistocene relict in a coastal salt marsh. Bulletin of the Florida State Museum of Biological Science 28(1-4): 2552. 
1187. Worah, S. 1990. Rusty spotted Cat Felis mubiginosa. P. 12. In: Anon, Cat Specialist Group meeting reports. Cor News 12: $2-14$.

1188. Wright, P.C. 1988. Lemurs' last stand. Animol Kingdom January/February 1988: 12-25.

1189. Ximenez, A. 1975. Felis geoffroyi. Mammalian Species (American Society of Mammalogists) 54: 1-4.

1190. Ximenez, A., Langguth, A. and Praderi, R. 1972. Lista sistematica de los mamiferos del Uruguay. Anales del Museo Nacional de Historia Natural de Montevideo $7(5): 1-49$

1191. Yalden, D.W., Largen, M.J. and Kock, D. 1976 Catalogue of the mammals of Ethiopia, 2. Insectivora and Rodentia. Monitore Zoologico Italiano (N.S.) 8(Suppl.): 1-118.

1192. Yalden, D.W., Largen, M.J. and Kock, D. 1977 Catalogue of the mammals of Ethiopia, 3. Primates. Monilore Zoologico lıoliano (N.S.). 9(Suppl.): 1-52.

1193. Yalden, D.W., Largen, M.J. and Kock, D. 1980. Catalogue of the mammals of Ethiopia, 4. Camivora. Monilore Zoologico Italiano (N.S.). 13(Suppl.): 170-272.

1194. Yang De-hus 1988. Black Snub-nosed Monkeys in China. Oryx 22: 41-43.

1195. Yasuma, S. 1988. Iniomote Cat: king of the night. Animal Kingdom Nov/Dec 1988: 12-21.

1196. Yleseev, N.V., (Ed.) 1983. Red Data Book of Russian SSR. Lesnaya Promiishlyennost, Moscow.

1197. Yom-tov, Y. and Ilani, G. 1987. The numerical status of Gazella dorcas and Gazella gazella in the southern Negev Desert, Israel. Biological Conservation 40(4): 245-253.
1198. Yoneda, M. 1984. An introduction to the mammalian fauna in Bolivia. Honyurui Kagaku 49: 21-40.

1199. Youngman, P.M. 1990. Mustela lutreolo. Mammalia 7 Species (American Socicty of Mammalogists) 362.

1200. Zarkhidze, V.A. 1983. TThe distribution and numbers of a population of Ovis vignei orcal in the south-west of its range.] Zoologicheskii Zhumal 62(11): 1756-1759. (In Russian).

1201. Zawisza, T. (in press). Guia ecologia de la fauns de Venezuela. Caracas, Venezuela.

1202. Zhang Yong-Zu, Wang Sung and Quan Guo-qiang 1981. On the geographical distribution of primates in China. Joumal of Human Evolution 10: 215-226.

1203. Zhou Kai-ya, Qian Wei-juan and Li Yue-min 1977. [Studies on the distribution of Baiji, Lipotes vexillifer Miller.] Acta Zoologica Sinica 23: 72-79. (In Chinese).

1204. Zhuge, Y. 1982. On the geographical distribution of the mammalian fauna of Zhejiang Province. Acla Theriologica Sinico 2(2): 157-166.

1205. Ziccardi, F. 1970. The African Wild Ass. Part 1. African Wildlife 24(3): 202-208.

1206. Ziccardi, F. 1970. The African Wild Ass. Part II. African Wildlife $24(4)$ : 287-292

1207. Zyll de Jong, C.G. Van 1972. A systematic review of the Nearctic and Neotropical river olters (genus Lutro). Contribution of the Life Science Division Royal Ontario Museum 80: 1-104. 
134 


\section{INDEX}

Includes scientific and common names; synonyms and names alternative to those used by CITES or the Red List are placed in parentheses.

Aardwolf 78

abyssinica, Genetta 75

(abyssinicus, Colobus) 34

Acerodan 11

Achdari 88

Acinonyx 78

acutarastrata, Balaenoptera 64

acutus, Lagemarhynchus 60

adamauae, Redunca fulvorufula 101

Addax 95

Addax 95

adersi, Cephalophus 98

admiralitatum, Pterapus 12

(aduncus, Tursiaps) 63

aedium, Plagiadantia 55

Aepyceras 95

aethiops, Cercopithecus 31

affinis, Rasufa 47

africana, Kerivaula 17

africana, Loxadonia 86

africanus, Equus 87

agilis, Cercocebus 31

(agilis, Cercocebus galeritus) 31

agilis, Hylabates 41

(agilis, Hylabates lar) 41

Agout 54

Ailuropoda 69

Ailunus 70

alaotrensis, Hapalemur griseus 20

albicans, Pithecia 30

albidens, Melamys 52

albifrans, Cebus 29

albifrons, Lemur fulvis 20

albigena, Cercocebus 31

albinasus, Chiropates 30

albipes, Conilurus 51

albirostris, Cervis 91

albirostris, Lagenarhynchiss 60

albocallaris, Lemur fulvus 21

(albagularis, Cercopithecus) 33

Alcelaphus 95

aldabrensis, Preropus seychellensis 15

alecta, Pterapus 12

alfredi, Cervus unicalor 93

(alfredi, Cervus) 93

slianycteris 11

(allamandi, Galicris) 72

allopaticala, Peramyscus gassypinus 50

alleni, Galago 24

sllenopithecus 31

Allocebus 22

allophrys, Peramyscus palianatus 50

Almiqui 5

Alouanta 27-28

(Alouana) 28

alpinus, Cuan 67

alsaica, Mustela 74

alticola, Peragnathus 48

Amblysamus 6

americana, Antilocapra 95

americanus, Ursus 69

ammabates, Peramyscus palianotus 50

Ammodarcas 95 ammon, Ovis 101

Ammotragus 95

amphibius, Hippopotamus 90

amplus, Notomys 52

ampullatus, Hyperaodon 56

Anathana 19

anetianus, Pteropus 12

angelcabrerai, Capromys 54

angalensis, Colabus 34

Angwantibo 23

(anhuiensis, Moschus) 94

(annamiticus, Axis parcinus) 93

annamiticus, Cervus parcinus 93

(annectens, Lutra) 72

Anoa, Lowland 96

Anos, Mountain 97

Anomalurus 49

ansellarum, Crocidura 7

Anteater, Banded 1

Antealer, Collared 42

Anteater, Giant 42

Anteater, Lesser 42

Antechinus 1

Antelope, Beirs 99

Antelope, Four-homed 102

Antelope, Giant Sable 100

Antelope, Tibetan 101

Antelope, Zebra 98

anchanyi, Neatoma 49

Antilocapra 95

Anrilope 96

antisensis, Hippocamelus 93

anubis, Papio hamadryas 38

(anubis, Papia) 38

Aanyx 71

Aatus 28

(Aatus) 28

Aoudad 95

Ape, Barbary 37

apella, Cebus 29

aphylla, Phyllanycteris 17

apicalis, Antechinus I

apicalis, Leporillus 51

(apicalis, Parantechinus) I

Apladantia 46

Aproteles 11

aquaticus, Hyemoschus 91

aquilo, Natamys 52

arachnoides, Brachyteles 29

Arctictis 75

Arciacebus 23

Arctocephalus 84-85

(Arctacephalus) 85

Arctogalidia 75

arcraides, Macaca 36

arctos, Ursus 69

Argali 101

Argali, Tibetan 101

argentata, Callithrix 25

argentatus, Oryzamys 50

(argentatus, Oryzomys palustris) 50

argentatus, Pterapus 12

Armadillo, Brazilian Three-banded 43
Armadillo, Burmeister's 43

Armadillo, Chacoan Fairy 43

Armadillo, Giant 43

Armadillo, Greater Naked-tailed 43

Armadillo, Lesser Fairy 43

Armadillo, Northern Naked-tailed 43

Armadillo, Pink Fairy 43

amee, Bubalus 96

amuxii, Berandius 56

(arquatus, Pteropus) 11

ascanius, Cercopithecus 32

(asinus, Equus) 87

asomatus, Lagorchestes 4

Ass, African 87

Ass, African Wild 87

Ass, Asiatic Wild 88

Ass, Incian Wild 88

Ass, Mongolian Wild 88

Ass, Onager 88

Ass, Syrian Wild 88

assamensis, Macaca 36

(Atelerix) 7

Ateles 28-29

aterrimus, Cercocebus albigena 31

(aterrimiss, Cercocebus) 31

athabascae, Bison bison 96

atrata, Dorcopsis 4

(atrimaculatus, Phalanger) 2

(atristriauts, Eutamias minimus) 47

alristriatus, Tamias minimiss 47

attenuata, Feresa 59

attenuata, Stenella 62

(attenuata, Stenella dubia) 62

(arys, Cercacebus) 31

aurantius, Rhinonicteris 17

aurata, Felis 79

(auratus, Trachypithecus) 38

aureus, Canis 66

aureus, Hapalemur 20

aurila, Callithrix jacchus 26

(aurila, Callithrix) 26

aurita, Geagale aurita 5

aurita, Myzopoda 17

aurius, Capramys 54

aurapunctatus, Herpestes 77

australis, Arctacephalus 84

australis, Balaena glacialis 66

australis, Dusicyan 67

(australis, Eubalaena) 66

australis, Lagenarhynchus 60

australis, Tamias quadrivillatus 47

(Australaphocaena) 63

(Avahi) 23

avunculus, Pygathrix 40

(avunculis, Rhinopithecus) 40

(Aris) 93

Aye-aye 23

(aygula, Presbytis) 38

(azarae, Aotius) 28

Babirusa 89

Baboon. Chacma 38

Baboon, Gelada 40

Baboon, Guinea 38 
Baboon, Hamadryas 38

Baboon, Olive 38

Baboon, Westem 38

Baboon, Yellow 38

Babyrousa 89

babyrussa, Babyrousa 89

bactrianus, Camelus 91

bactrianus, Cervus elaphus 91

Badger, Honey 73

badia, Felis 79

badius, Colobus 34

(badius, Procolobus) 34

baileyi, Crocidura 8

baileyi, Nemarhaedus goral 100

(baileyi, Nemorhaedus) 100

bairdii, Berardius 56

bairdii, Tapirus 88

Balaena 65-66

Balaenoptera 64-65

balutus, Pieropus 12

(balutus, Pheropus pumilus) 12

bancanus, Tarsius 25

Bandicoot, Barred 2

Bandicoot, Clara 2

Bandicoot, Desert 2

Bandicoot, Greater Rabbit-eared 2

Bandicoot, Lesser Rabbit-eared 2

Bandicoot, Pig-footed 2

Bandicoot, Westem Barred 2

Bandicoot, White-lipped 2

(banteng, Bos) 96

Bonteng 96

Borasingha 91

barbara, Eira 71

barbara, Hyaena hyaena 78

barbarus, Cenvus elaphus 92

barbatus, Xenuramys 53

(bamardi, Lasiorhinus) 3

barrerae, Octomys 55

(barrerae, Tympanoctomys) 55

barelsi, Myotis formosus 18

(bartelsi, Myoris) 18

Bassaricyon 70

Bat, Big Long-nosed 17

Bat, Bulmer's Fruit 11

Bat, Chapman's Fruit 11

Bat, Cuban Flower 17

Bat, Dayak Fruit 11

Bal, Geelvinck Bay Leaf-nosed 16

Bat, Ghost 16

Bat, Greater Sheath-tailed 16

Bat, Grey 18

Bat, Herman's 18

Bat, Indiana 18

Bat, Jamaican Flower 17

Bat, Javan Mastiff 18

Bat, Javan Tail-less Fruit 11

Bat, Javan Thick-thumbed 17

Bat, Kitti's Hog-nosed 16

Bat, Large Mouse-eared 18

Bat, Lesser Naked-backed Fruit 11

Bat, Litule Long-nosed 17

Bat, Long-fingered 17

Bat, Mexican Long-nosed 17

Bat, New Zealand Lesser Short-tailed 18

Bat, Orange Horseshoe 17

Bat, Orange Leaf-nosed 17

Bat, Philippines Tube-nosed Fruit 12

Bat, Pond 18
Bat, Puerto Rican Flower 17

Bat, Raffray's Sheath-tailed 16

Bat, Ridley'a Leaf-nosed 16

Bat, Seychelles Sheath-tailed 16

Bat, Singapore Roundleaf Horseshoe 16

Bat, Social 18

Bat, Sucker-footed 17

Bat, Tanzanian Woolly 17

Bat, Townsend's Bigeared 18

Bat, White-lined 17

Batomys 51

bavaricus, Pitymys 51

Bdeogale 77

Bear, American Black 69

Bear, Asiatic Black 69

Bear, Baluchistan 69

Bear, Himalayan Brown 70

Bear, Polar 70

Bear, Sloth 69

Bear, Spectacled 69

Bear, Sun 69

Beaver, Broad-tail 49

Beaver, Point Arena Mountain 46

Beaver, Point Reyes Mountain 46

Beaver, Rio Grande 49

bedfordi, Budorcas taxicolor 97

beecrofii, Anomalurus 49

(belangeri, Tupaia) 19

Beluga 58

belzebul, Alouana 27

belzebuth, Areles 28

bengalensis, Felis 79

bengalensis, Vulpes 68

(beniensis, Alouatta) 28

(benneti, Gazella) 99

bennetianus, Dendrolagus 3

bennertii, Cynagale 75

Berardius 56

berezovskii, Moschus 93

(berezovskii, Maschus chrysogaster) 93

beringei, Gorilla gorilla 42

Bettong, Brush-tsiled 3

Bettong, Burrowing 3

Bettong, Eastern 3

Bettong, Tasmanian 3

Bettongia 3

(Bettongia) 3

(bezoarcticus, Odocoileus) 94-95

bezoarticus, Ozoloceros 94

bicolor, Ralufa 47

bicolor, Saguinus 26

bicomis, Diceros 89

bidens, Mesoplodon 57

biet, Felis 79

bieti, Pygathrix roxellana 40

(bieti, Rhinopithecus) 40

Bilby, Greater 2

Bilby, Lesser 2

binturong, Arctictis 75

birulai, Castor fiber 49

Bison 96

Bison, European 96

Bison, Indian 96

Bison, Wood 96

bisulcus, Hippocamelus 93

Blackbuck 96

Blackfish, Slender 59

blainvillei, Pontoporia 56

Blastocerus 91
Blesbok 99

Bluebuck 100

Bobcat 82

(boliviensis, Bradypus) 43

(boliviensis, Saimiri) 30

bonasus, Bison 96

(bonasus, Bison bison) 96

Bongo 96

Bonobo 42

Bontebok 99

Boocercus 96

Boodie 3

borealis, Balaenoptera 64

borealis, Lissodelphis 61

(bomeensis, Sousa) 62

Bos 96

Bottlehead 56

bougainville, Perameles 2

Boutu 55

(bouvieri, Colobus badius) 34

bouvieri, Colobus pennantii 34

(bouvieri, Procolobus pennantii) 34

bowdoini, Mesoplodon 57

Brachyteles 29

brachyurus, Chrysocyan 67

Bradypus 43

(Bradypus) 43

branickii, Dinomys 54

brasiliensis, Pteronura 74

(brasiliensis, Sotalia) 62

bredanensis, Steno 63

brelichi, Pygathrix 40

(brelichi, Rhinopithecus roxellana) 40

(brelichi, Rhinopithecus) 40

brevicaudata, Chinchilla 54

brevicaudata, Microgale 5

breviceps, Kogia 58

brevinasus, Perognathus longimembris 48

brevirostris, Orcaella 61

breweri, Microtus 51

(breweri, Microtus pennsylvanicus) 51

brochus, Syntheosciurus 47

Brocket, Middle American Red 93

brooki, Dyacopterus spadiceus 11

(brooki, Dyacopterus) 11

(brownii, Capromys) 55

brownii, Geocapromys 55

briijni, Zaglossus 1

(brumbacki, Aotus) 28

brunnea, Hyaena 78

brunnescers, Macaca ochreata 37

(brunnescens, Macaca) 37

(brunneus, Callicebus) 29

brunneus, Pieropus 12

Bubalus 96-97

Budorcas 97

Buffalo, Wild Asiatic 96

bulmerae, Aproteles 11

bunkeri, Neotoma 49

Bunolagus 45

(Burmeisteria) 43

Burramys 3

Bushhaby, Allen's 24

Bushbaby, Dwarf 24

Bushhaby, Eastem Needle-clawed 24

Bushbaby, Grant's 24

Bushbaby, Greater 25

Bushbaby, Thick-tailed 25

Bushhaby, Western Needle-clawed 24 
Bushbaby, Zanzibar 25 buxtoni, Trogelaphus 102 Cabassous 43 Cacajao 29

Cachelot 58

Cacomistle, Central American 70 caissora, Leontopithecus rosolia 26 (caissora, Leontopithecus) 26 calabarensis, Arctocebus 23 (calamianensis, Axis) 93 calamianensis, Cervus porcinus 93 Calcochloris 7

(caligotus, Callicebus) 29

Callicebus 29

(Callicebus) 29

Callimico 27

Collithrix 25-26

(Callithrix) 26

Caloprymnus 3

calura, Phascogole 1

calvus, Cacajao 29

Camel, Wild Bactrian 91

Camelus 91

campbelli, Cercopithecus 32

compestris, Coloprymnus 3

cana, Vulpes 68

canadensis, Felis 79

(canadensis, Felis lynx) 79

canadensis, Lutro 72

(canadensis, Lynx) 79

canadensis, Oris 101

caniceps, Pheropus 12

Canis 66-67

capaccinii, Myotis 17

copensis, Aonyx 71

capensis, Mellivoro 73

Caperea 66

Capra 97

(Capra) 97

Capricomis 97-98

Caprolagus 45

Capromys 54-55

(Capromys) 55

Capuchin, Black-capped 29

Capuchin, Brown Pale-fronted 29

Capuchin, Tufted 29

Capuchin, Weeper 29

Capuchin, While-faced 29

Capuchin, White-fronted 29

Capuchin, White-throated 29

copucinus, Cebus 29

caracal, Felis 79

(caracal, Lynx) 79-80

Caracal, African 79

Caracal, Asian 79

Caracal, Turkmenian 80

caraya, Alouatra 28

carthubbsi, Mesoplodon 57

(carpentarius, Notomys) 52

cartilogonodus, Otopteropus 12

cartusiana, Rupicopra rupicapro 101

(castaneus, Conepalus) 71

Castor 49

Cal, African Golden 79

Cal, Andean 80

Cat, Asiatic Golden 82

Cat, Bay 79

Cat, Black-footed 81

Cat, Bomean Martled 79
Cat, Chilean 80

Cat, Chilean Pampa 80

Cat, Chinese Desen 79

Cat, Fishing 82

Cat, Flat-headed 81

Cat, Geoffroy's 80

Cat, Golden 79

Cat, 1riomote 80

Cat, Jungle 80

Cat, Leopard 79

Cat, Little Spotted 82

Cat, Marbled 81

Cat, Mountsin 80

Cat, Pakistan Sand 81

Cat, Pallas's 81

Cat, Pampas 80

Cat, Reed 80

Cat, Sand 81

Cat, Small-spotted 81

Cat, Swamp 80

Cat, Temminck's 82

Cat, Tiger 82

Cat, Wild 82

Cotogonus 90

(cotodon, Physeter) 58

conta, Lemur 20

coudata, Marmota 46

cavirostris, Ziphius 58

cebifrons, Sus borbolus 90

Cebuello 26

Cebius 29

(Cebus) 29

celebensis, Acerodon 11

(celer, Odocoileus bezoarcicicus) 95

celer, Ozoroceros bezoarticus 95

centrolis, Cobassous 43

Cephalophus 98

Cephalorhynchus 59

cephus, Cercopithecus 32

cerasina, Mazamo omericana 93

Cerototherium 89

Cercocebus 31

Cercopithecus 31-33

(Cercoptihecus) 31-33

cerrosensis, Odocoileus hemionus 94

cervicopra, Antilope 96

Cervus 91-93

Choeropus 2

Chaetomys 53

Chamois, Abruzzo 102

Chamois, Tatra 102

chopmani, Dobsonio exoleto II

(chapmani, Dobsonia) 11

chaus, Felis 80

Cheetah, Asiatic 78

Cheirogaleus 22

(Cheirogaleus) 22

Chevrolain, Water 91

chihuohuensis, Microus pennsylvanicus 51

Chimparzee 42

Chimpanzee, Dwarf 42

Chimpanzee, Pygmy 42

Chimpanzee, West African 42

Chinchilla 54

Chinchilla, Long-tailed 54

Chinchilla, Short-tailed 54

chinensis, Sousa 62

Chingungo 72

Chiropotes 30
Chiru 101

Chlamyphorus 43

Chlorotalpo 7

(Chlorololpo) 7

Choeropsis 90

Choloepus 43

Chrologale 75

Chrysochloris 7

Chrysocyon 67

chrysogaster, Moschus 94

chrysoleuca, Callithrix humeralifer 26

chrysomelas, Leontopichecus rosalia 26

(chrysomelas, Leontopithecus) 26

chrysoproctus, Pleropus 12

chrysopygus, Leontopithecus rosalia 26

(chrysopygus, Leoniopithecus) 26

chrysopygus, Rhynchocyon 10

Chrysospalax 7

chrysospilo. Mortes flavigula 73

Ciego, Greater Pichi 43

Ciego, Lesser Pichi 43

(cinerascens, Collicebus) 29

cinerea, Aonyx 71

cinereus, Lasiurus 17

cinereus, Sciurus niger 47

cimei, Rhynchocyon cimei 10

Civet, African 75

Civet, Banded Palm 76

Civet, Brown Palm 76

Civet, Javan Small-toothed Palm 75

Civet, Jerdon's Palm 76

Civet, Malabar Large-spotted 76

Civet, Malagasy 75

Civet, Owston's Palm 75

Civet, Striped 75

Civet, Sulawesi Palm 76

civetta, Civetrictis 75

(civello, Viverro) 75

Civettictis 75

civettina, Viverra megaspila 76

(civerina, Viverra) 76

clara, Echymipero 2

clorkei, Ammodorcas 95

clavium, Odocoileus virginionus 94

clymene, Stenella 62

Coati, Cozumel Island 70

Coati, South Brazilian 70

Cochito 64

(Coendou) 54

coeruleoalba, Stenella 62

Coleura 16

collaris, Lemur fulvus 21

Colobus 34-35

Colobus, Angolan 34

Colobus, Angolan Black-and-White 34

Colobus, Black 35

Colobus, Bouvier's Red 34

Colobus, Eastem Black-and-White 34

Colobus, Eastern Red 34

Colobus, Elliot's Red 34

Colobus. Foa Red 34

Colobus, Gordon's Red 34

Colobus, King 35

Colobus, Kirk's 35

Colobus, Magistrate 34

Colobus, Miss Waldron's Bay 34

Colobus, Olive 35

Colobus, Oustalet's Red 35

Colobus. Preuss's 35 
Colobus, Preuss's Red 35

Colobus, Tana River 35

Colobus, Temminck's Red 34

Colobus, Thollon's Red 35

Colobus, Uganda Red 35

Colobus, Uhehe Red 34

Colobus, Van Beneden's 35

Colobus, Western Black-and-White 35

Colobus, Western Red 34

(Colobus) 35

colocolo, Felis 80

coloratus, Glaucomys sabrinus 46

comata, Presbytis 38

commersonii, Cephalorhynchus 59

concolor, Felis 80

concolor, Hylobates 41

concolor, Nasalis 37

concolor, Salanoia 78

(concolor, Simias) 37

conditor, Leporillus 51

Conepatus 71

(Conepalus) 71

Coney, Indian 55

congica, Aonyx 71

congobelgica, Crocidura 8

Conilurus 51

conspicillatus, Pteropus 12

coquereli, Microcebus 22

(coquereli, Mirza) 22

coronatus, Lemur 20

(coronatus, Lemur mongoz) 20

(coronatus, Penenus) 20

corsac, Vulpes 68

corsicanus, Cervus elaphus 92

coryi, Felis concolor 80

costaricensis, Felis concolor 80

cottoni, Ceratatherium simum 89

Cottontail, Omilteme 45

Cottontail, Tres Marias 45

coucang. Nycticebus 24

Cougar 80

Cougar, Eastern 80

Cougar, Florida 80

couguar, Felis concolor 80

(cranbrooki, Nemorhaedus) 100

Craseonycteris 16

crassicaudata, Manis 44

crassicaudatus, Otolemur 25

crassidens, Pseudorca 61

crassipes, Microgale 5

crenala, Crocidura 8

crinifrons, Muntiacus 94

cristata, Hystrix 53

cristata, Presbytis 38

cristatus, Proteles 78

(cristatus, Trachypithecus) 38

Crocidura 7-9

cnuciger, Lagenorhynchus 60

crusalbum, Cephalophus ogilbyi 98

Cryprochloris 7

Cryproprocia 75

cubanus, Solenadon 5

(culpaeolus, Dusicyon) 67

culpaeus, Dusicyon 67

(Cuniculus) 54

Cuon 67

(cupreus, Callicebus) 29

Cuscus, Black-spotted 2

Cuscus, Common Spotted 2
Cuscus, Grey 2

Cuscus, Silky 2

Cuscus, Woodlark lsland 2

cuvieri, Gazella 99

cyclopis, Macaca 36

(cynamolgus, Macaca) 36

cynocephalus, Papio hamadryas 38

(cynocephalus, Papio) 38

cynocephalus, Thylacinus 1

Cynogale 75

(Cynogale) 75

Cynomys 46

Dalgyte 2

dalli, Phocoenoides 64

dama, Gazella 99

(Dama) 91

Damaliscus 99

dammah, Oryx 101

Dassie, Eastern Tree 86

dasycneme, Myotis 18

dasymallus, Pteropis 12

Dasyprocta 54

Daubentonia 23

davidianus, Elaphunus 93

dealbata, Ratufa indica 47

decemlineata, Mungotictis 78

Deer, Argentinian Pampas 95

Deer, Atlas 92

Deer, Bactrian 91

Deer, Bactrian Red 91

Deer, Barbary 92

Deer, Barbary Red 92

Deer, Bokharan 91

Deer, Calamian Hog 93

Deer, Cedros Island Mule 94

Deer, Cedros Island Black-tailed 94

Deer, Chinese Water 93

Deer, Corsican Red 92

Deer, Dwarf Musk 93

Deer, Indochinese Hog 93

Deer, Kerama 92

Deer, Key Deer Toy 94

Deer, Kuhl's Hog 93

Deer, MacNeill's Red 92

Deer, Manipur Brow-antlered 92

Deer, Marsh 91

Deer, Pampas 94

Deer, Persian Fallow 91

Deer, Siberian Musk 94

Deer, South Ching Forest Musk 93

Deer, Swamp 9]

Deer, Thorold's 91

Deer, White-lipped 91

Deer, Yarkand 92

Delphinapterus 58

Delphinus 59

delphis, Delphinus 59

(demidof, Galagoides) 24

demidovii. Galago 24

Dendrogale 19

Dendrohyrar 86

Dendrolagus 3-4

densirosinis, Mesoplodon 57

(denti, Cercoptihecus) 33

deppei, Sciunus 47

(depressicomis, Anoa) 96

depressicomis, Bubalus 96

derbianus, Anomalunus 49

(derbionus, Taurotragus derbianus) 102 (derbianus, Tragelaphus derbianus) 102

derbianus, Trogelaphus ony 102

derbyanus, Hemigalus 76

Desman, Pyrenean 10

Desman, Russian 10

Desmana 10

Dhole 67

diadema, Propithecus 23

diana, Cercopithecus 32

diazi, Romerolagus 45

Dibatag 95

Dibbler 1

Dicerorhinus 89

Diceros 89

dichotomus, Blastocerus 91

(Didemocent) 89

Dinomys 54

(dioptrica, Australophocaena) 63 dioptrica. Phocoena 63

Dipodamys 48

Dobsonia 11

Dog, African Hunting 68

Dog, Asjatic Wild 67

Dog, Bush 68

Dog, Mexican Prairie 46

Dog, Red 67

Dog, Small-eared 67

Dog, Utah Prairie 46

Dog, Wild 68

Dolphin, Amazon River 55

Dolphin, Atlantic 59

Dolphin, Atlantic Spinner 62

Dolphin, Atlantic White-sided 60

Dolphin, Benguela 59

Dolphin, Black 59

Dolphin, Blackchin 60

Dolphin, Bottle-nosed 63

Dolphin, Bridled 62

Dolphin, Changjiang 56

Dolphin, Chilean 59

Dolphin. Clymene 62

Dolphin, Commerson's 59

Dolphin, Common 59

Dolphin, Dusky 61

Dolphin, Estuarine 62

Dolphin, Euphrosyne 62

Dolphin, Fraser's 60

Dolphin, Ganges River 56

Dolphin, Grey 60

Dolphin, Guianian River 62

Dolphin. Heaviside's 59

Dolphin, Hector's 59

Dolphin, Helmet 62

Dolphin, Hourglass 60

Dolphi $\pi$. Indopacific Humpback 62

Dolphin, Indus 56

Dolphin, Irrawaddy 61

Dolphin, La Plata River 56

Dolphin, Long-beaked 63

Dolphin, Long-snouted 63

Dolphin, Narrow-snouted 62

Dolphin, New Zealand 59

Dolphin, Pacific 59

Dolphin, Pacific White-sided 61

Dolphin, Pantropical Spotted 62

Dolphin, Peale's 60

Dolphin, Piehald 59

Dolphin. Risso's 60

Dolphin. Rough-toothed 63 
Dolphin, Saddle-backed 59

Dolphin, Sarawak 60

Dolphin, Snubfin 61

Dolphin, Spinner 62, 63

Dolphin, Striped 62

Dolphin, Tropical 59

Dolphin, White-beaked 60

Dolphin, White-bellied 59

Dolphin, White Flag 56

Dolphin, White-headed 59

Dolphin, Whitefin $\mathbf{5 6}$

(donacophilus, Callicebus) 29

dorces, Damaliscus 99

dorcas, Damaliscus dorcas 99

dorcas, Gazella 99

Doncatragus 99

Dorcopsis 4

Dorcopsis, Macleay'a 4

Dorcopsis, Papuan 4

(Dorcopsulus) 4

dorsalis, Cephalophus 98

(dorsalis, Lemur mustelinus) 21

dorsalis, Lepilemur 21

dorsalis, Tupaia 19

Douc 40

douglasi, Sminthopsis 1

Douroucouli 28

Drill 38

drowhardi, Microgole 5

dryas, Cercopichecus 32

(dubia, Stenella) 62

(dubius, Callicebus) 29

dugon. Dugong 87

Dugong 87

Duiker, Abbott's 98

Duiker, Aders' 98

Duiker, Banded 98

Duiker, Bay 98

Duiker, Blue 98

Duiker, Jentink's 98

Duiker, Ogilby's 98

Duiker, Ruwenzori Black-fronted 98

Duiker, White-legged 98

Duiker, Yellow-backed 98

Duiker, Zebra 98

dukecampbelli, Microlus pennsylvanicus 51

Dunnart, Julia Creek 1

Dunnart, Long-tailed 1

Dunnart, Sandhill 1

Dusicyon 67

(Dusicyon) 67

duthiae, Chlorotalpa 7

duvauceli, Cervus 91

Dyacopterus 11

Dziggetai 88

ebii, Epixerus 46

ecaudatus, Chaeropus 2

Echidna, Long-beaked 1

Echidna, Long-nosed 1

Echymipera 2

edeni, Balaenoptera 64

(edwardii, Clobicephala) 60

edwardsi, Herpesles 77

(edwardsi, Lemur mustelinus) 21

edwardsi, Lepilemur 21

Eira 71

eisentrouti, Crocidura 8

eisentrauti, Myosorex 9

Eland, Eastern Giant 102
Eland, Westem Giant 102

Elaphunus 93

elavor, Dipodomys 48

eldi, Cervus 92

eldi, Cernus eldi 92

electra, Peponocephala 61

(elegans, Arciocephalus) 85

elegans, Macruromys 51

(elegantulus, Euoticus) 24

elegantulus, Galago 24

Elephant, African $\mathbf{8 6}$

Elephant, Asian 86

Elephant, Indian 86

Elephant-seal, Southem 85

Elephant-shrew, Black-and-rufous 10

Elephant-shrew, Chequered 10

Elephant-shrew, Four-toed 10

Elephant-shrew, Golden-rumped 10

Elephant-shrew, Henderson's Chequered 10

Elephant-shrew, Yellow-rumped 10

Elephas 86

ellioti, Anathana 19

(ellioti, Colobus badius) 34

ellioti, Colobus pennantii 34

(ellioti, Procolobus rufomilratus) 34

elphinstoni, Ratufa indica 47

Emballonura 16

emous, Thomomys umbrinus 48

Enhydra 71-72

eniellus, Presbyris 39

(entellus, Semnopinhecus) 39

(enudris, Lutra) 72

Epixerus 46

Equus 87-88

(Equus) 88

eremiana, Perameles 2

Eremilalpa 7

Erinaceus 7

Ermine 74

erminea, Mustela 74

Erythrocebus 35

eryihrogaster, Cercopithecus 32

enthrotis, Cercopithecus 32

Eschrichtius 64

(Eubalaena) 65-66

(Euoricus) 24

Eupleres 75

(Eupleres) 75

europaeus, Mesoplodon 57

eurycerus, Boocercus 96

(eurycerus, Tragelaphus) 96

(Eulamias) 47

eutropia, Cephalorhynchus 59

evereti, Melogale 74

evereni, Urogale 20

exilis, Dipodomys nitraloides 48

Falanouc 75

falconeri, Capra 97

fasciata, Galidicris 77

fasciatus, Lagostrophus 4

fascialus, Mymecobius 1

fascicularis, Macaca 36

fauniclus, Pteropiss 12

feai, Muntiacus 94

felina, Luira 72

Felis $79-83$

(Felis) 80,82

(Jemoralis, Presbytis) 39

(Fennecus) 68
Feresa 59

ferox, Cryptoprocia 75

Fernet-badger, Everett's 74

Ferret-badger, Javan 74

Ferret-badger, Kinabalu 74

Ferret, Black-footed 74

(ferus, Camelus) 91

fieldi, Pseudomys 52

Finback 65

Fish, Devil 64

flavicauda, Lagothrix 30

flaviceps, Callithrix jacchus 26

(flaviceps, Callithrix) 26

flavifrons, Lemur macaco 21

Ravigula, Martes 73

Ravigularis, Lepus 45

flavus, Potos 70

fuviatilis, Sotalia 62

Flying-fox, Admiralty 12

Flying-fox, Aldabra 15

Flying-fox, Big-eared 13

Flying-fox, Greater Mascarene 14

Flying-iox, Guam 15

Flying-fox, Large Palau 14

Flying-fox, Micronesian 13

Flying-fox, Mortlock 14

Flying-fox, Pohnpei 14

Flying-fox, Rodrigues 15

Flying-fox, Silvery 12

Flying-fox, Truk 13

Flying-Fox, Insular 15

(foai, Colobus badius) 34

foci, Colobus pennantii 34

(foai, Procolobus rufomitralus) 34

Forest-wallaby, Black 4

Forest-wallaby, Papuan 4

formosus, Otomops 18

forsteri, Arciocephalus 84

fossa, Fossa 75

Fossa 75

Fosse 75

(fossana, Fossa) 75

Fox, Afghan 68

Fox, Argentine Grey 67

Fox, Bengal 68

Fox, Blanford's 68

Fox, Colpeo 67

Fox, Corsac 68

Fox, Fennec 68

Fox, Forest 67

Fox, Hoary 67

Fox, Island Grey 68

Fox, Pale 68

Fox, Pampa 67

Fox, Rueppell's 68

Fox, Sand 68

Fox, Sechura 67

Fox, Simien 67

fraenata, Onychogalea 4

Franciscana 56

francoisi, Presbytis 39

(francoisi. Trachypithecus) 39

frondainr, Castor canadensis 49

(frontalis, Alelerix) 7

(frontalis, Bos) 96

frontalis, Erinaceus 7

frontalis, Stenella 62

(frontalis, Stenella attenuala) 62

frontala, Presbytis 39 
frontotus, Aleles geaffroyi 28

fulgens, Ailurus 70

(fulvipes, Dusicyon) 67

fulvus, Lemur 21

fulvus, Lemur fulvus 21

fumeus, Pseudomys 52

fundatus, Pteropus 12

furax, Emballonura 16

furcifer, Phaner 23

fumessi, Pentalagus 45

fusca, Alouatra 28

fuscala, Macaca 36

fusciceps, Ateles 28

fuscicallis, Saguinus 27

fuscus, Glaucomys sabrinus 46

fuscus, Herpesies 77

fuscus, Moschus 94

(fuscus, Mosehus chrysagaster) 94

fuscus, Notomys 52

gabbii, Bassaricyon 70

gaimardi, Bettongia 3

Galago 24-25

Galago, Allen's 24

Galago, Demidofrs 24

Galago, Eastem Needle-clawed 24

Galago, Grant's 24

Galago, Westem Needle-clawed 24

Galago, Zanzibar 25

(Galago) 24-25

(Galagoides) 24

galapagaensis, Arctocephalus 84

Galemys 10

galeritus, Cercacebus galeritus 31

Galictis 72

(Galictis) 72

Galidicris 77

Gama 95

gangetica, Platanisto 56

(gametri, Otolemur) 25

garridoi, Capromys 54

Gaur 96

gaurus, Bos 96

gazella, Arcucephalus 84

gazella, Gazella 99

Gazella 99-100

(Gazella) 99

Gazelle, Addra 99

Gazelle, Arabian Goitred 100

Gazelle, Clarke's 95

Gazelle, Cuvier's 99

Gazelle, Dama 99

Gazelle, Dorcas 99

Gazelle, Edmi 99

Gazelle, Red-fronted 100

Gazelle, Sand 99

Gazelle, Slender-homed 99

Gazelle, Soemmerring's 100

Gazelle, Speke's 100

geala, Myosorex 9

gedrasianus, Selenarctos thibetanus 69

(gedrosianus, Ursus thibetanus) 69

geei, Presbytis 39

(geei, Trachypithecus) 39

gelado. Theropithecus 40

Genet, Abyssinian 75

Genet, Ibiza 75

Genet, Johnston's 76

Genena 75-76

Geocapramys 55
(Geocapromys) 55

geoffrensis, Inia 55

geaffroyi, Akeles 28

(geaffroyi, Callithrix) 26

geoffroyi, Felis 80

(geaffroyi, Sanguinus) 27

Geogale 5

Gibbon, Agile 41

Gibbon, Black 41

Gibbon, Bomean 41

Gibbon, Capped 41

Gibbon, Common 41

Gibbon, Crested 41

Gibbon, Crowned 41

Gibbon, Dark-handed 41

Gibbon, Dwarf 41

Gibbon, Grey 41

Gibbon, Hoolock 41

Gibbon, Indochinese 41

Gibbon, Javan 4]

Gibbon, Kloss's 41

Gibbon, Lar 41

Gibbon, Mentawai 41

Gibbon, Pileated 41

Gibbon, Potto 24

Gibbon, Silvery 41

Gibbon, White-cheeked 41

Gibbon, White-handed 41

(gibbosus, Eschrichtius) 64

gigantea, Manis 44

(gigantea, Phataginus) 44

(giganteus, Priodontes) 43

giganteus, Pterapus 12

gigas, Hydrodamalis 87

gigas, Macroderma 16

(gigas, Taurotragus derbianus) 102

gigas, Tragelaphus oryx 102

(gilli, Tursiaps) 63

gilliardi, Pteropus 13

giluwensis, Ranus 53

ginkgadens, Mesopladon 57

glacialis, Balaena 65

(glacialis, Eubalaena) 65

(glacialis, Orcinus) 61

glassi, Crocidura 8

Glaucamys 46

glis, Tupaia 19

Glischropus 17

Globicephala 60

(Globicephala) 60

gloveralleni, Procyan 71

(gloveralleni, Procyon lotar) 71

goeldii, Callimico 27

goldmani, Glaucomys volans 46

goliath, Crocidura odorata 9

Gopher, Michoacan Pocket 48

garal, Nemorhaedus 100

Goral 100

Goral, Red 100

(gardanorum, Colobus badius) 34

gardonorum, Colobus pennantii 34

(gardanarum, Procolobus) 34

garilla, Gorilla 41

Gorilla 41-42

Gorilla $41-42$

Gorilla, Eastem Lowland 42

goudorit, Eupleres 75

gouldii, Pseudomys 52

gracilis, Microgale 5 gracilis, Tupaia 19

(graellsi, Saguinus) 27

(graffmani, Stenella) 62

grahamensis, Tamiasciurus hudsonicus 47

Grampus 60

grandidieri, Galidictis 77

granti, Batomys 51

granti, Eremitalpa 7

granti, Galago 24

(granti, Galago senegalensis) 24

(granti, Galagoides zanaibaricus) 24

grassei, Crocidura 8

grassianus, Cervus nippon 92

graueri, Gorilla garilla 42

graueri, Paracrocidura 9

gravipes, Dipodamys 48

grayi, Mesoplodon 57

graysoni, Sylvilagus 45

grevyi, Equus 88

greyi, Macropus 4

griffichi, Vulpes vulpes 68

grisescens, Myotis 18

(griseus, Bradypus) 43

griseus, Dusicyon 67

griseus, Grampus 60

griseus, Hapalemur griseus 20

griseus, Pteropus 13

Grison, Greater 72

(gnunniens, Bos) 96

Guanaco 91

guanicoe, Lama 91

(guariba, Alouatta) 28

Guenon, Campbell's 32

Guenon, Crowned 33

Guenon, Diana 32

Guenon, Dryas 32

Guenon, Golden-bellied 33

Guenon, Lesser Spot-nosed 33

Guenon, Lesser White-nosed 33

Guenon, Owl-faced 32

Guenon, Preuss's 32

Guenon, Red-bellied 32

Guenon, Russet-eared 32

Guenon, Salongo 33

Guenon. Schmidi's 32

Guenon. Spot-nosed 33

Guenon, Sun-tailed 33

Guenon, White-nosed 33

Guenon, White-throated 32

guereza, Colobus 34

Guereza 34

guerreroensis, Glaucomys volans 46 (guianensis, Sotalia) 62

guigna, Felis 80

gula, Gulo 72

Gulo 72

gundlachi, Capromys 55

gurningi, Amblysomus 6

gwatkinsi, Manes flavigula 73

(gwalkinsi, Martes) 73

Gymnobelideus 3

gymnocercus, Dusicyon 67

Gymnure, Mindanao 7

(gymnurus, Cabassous) 43

hamadryas, Papio hamadryas 38

hamlyni, Cercopithecus 32

hanglu. Cervus elophus 92

Hangul 92

Hapalemur 20 
Hare, Hispid 45

Hare, Tehuantepec 45

Hare-wallaby, Banded 4

Hare-wallaby, Central 4

Hare-wallaby, Eastern 4

Hare-wallaby, Least 4

Hare-wallaby, Rufous 4

Hare-wallaby, Westem 4

Hantebeest, Hunter's 99

Hartebeest, Swayne's 95

Hartebeest, Tora 95

hartmannae, Equus zebra 88

heavisidii, Cephalarhynchus 59

hecki, Macaca tonkeana 37

(hecki, Macaca) 37

hectori, Cephalorhynchus 59

hecsori, Mesapladon 57

Hedgehog, South Africat! 7

hefneri, Sylvilagus palustris 45

Helarcias 69

Hemigalus 76

hemianus. Equus 88

hemionus, Equus hemianus 88

hemippus, Equus hemionus 88

(hemippus, Equus anager) 88

Hemitragus 100

(hendeei, Lagathrix) 30

hendersani, Rhynchacyan cimei 10

hermani, Myatis formosus 18

(hermani, Myatis) 18

hermaphroditus, Paradaxurus 76

Herpestes 77

himalayana, Marmata 46

Hippocamelus 93

Hippapatamus 90

Hippopotamus 90

Hippopotamus, Pygmy 90

Hippasideras 16

Hippotragus 100

Hirola 99

hirsuto, Pithecia 30

hirsutus, Lagarchestes 4

hispidus, Caprolagus 45

hodgsani, Panthalops 101

hodgsonii, Ovis ammon 101

haffmanni, Chaloepus 43

(haffmannsi, Callicebus) 29

Hog, Pygmy 90

haalock, Hylabates 41

Hoppirg-mouse, Big-eared 52

Hopping-mouse, Darling Downs 52

Hopping-mouse, Dusky 52

Hopping-mouse, Long-tailed 52

Hopping-mouse, Northern 52

Hopping-mouse, Short-tailed 52

Horse, Przewalski's 88

hasei, Lagenadelphis 60

hasei, Presbytis 39

(hosei, Prestytis comala) 39

howelli, Sylvisorex 10

hawensis, Pherapus 13

Howler, Black 28

Howler, Black-and-red 27

Howler, Brown 28

Howler, Guatemalan 28

Howler, Mantled 28

Howler, Red 28

Howler, Red-hatted 27

hualpaiensis, Microtus mexicanus 51
Huemul, Chilean 93

Huemul, North Andean 93

Huemul, Peruvian 93

Huemul, South Andear 93

Huillin 73

humboldrii, Caneparus 71

humeralifer, Callithrix 26

humilis, Acerodon 11

hunteri, Damaliscus 99

Hutia, Bahamian 55

Hutia, Brown's 55

Hutia, Bushy-tailed 55

Hutia, Cabrera's 54

Hutia, Chapman's Prehensile-tailed 55

Hutia, Cuvier's 55

Hutia, Dwarf 55

Hutia, Garrido's 54

Hutia, Hispaniolan 55

Hutia, Ingraham's 55

Hutia, Jsla de la juventud Tree 55

Hutia, Jamaican 55

Hutia, Land 55

Hutia, Large-eared 54

Hutia, Little Earth 55

Hyaena 78

Hyaena, Barbary 78

Hyaena, Brown 78

(Hydrictis) 73

Hydrodamalis 87

Hydropoles 93

Hyemaschus 91

(hylacum, Plagiodonia) 55

Hylobates 41

(Hylabates) 41

hylocrius, Hemitragus 100

Hyperoadon 56

(hypoleucos, Semnopithecus) 39

hypomelanus, Prerapus 13

Hystrix 53

Ihex, Pyrenean 97

Ibex, Walia 97

ldiunss 49

Idmi 99

Impala, Black-faced 95

imperalor, Saguinus 27

(incarum, Lutra) 72

(indi, Plotanista) 56

indica, Ratufa 47

indica, Viverricula 77

indicus, Tapinus 88

Indopacelus 56

indri, Indri 23

Indri 23

Indris 23

inemis, Hydrapoles 93

(infulatus, Aolus) 28

(infuscatus, Bradypus) 43

ingens, Dipodamys 48

ingrahami, Geacapromys 55

Inia 55

insonus, Sylvilagus 45

insularis, Pterapus 13

intermedia, Martes foina 73

intermedius, Callithrix humeralifer 26

inunguis, Trichechus 87

inustus, Dendrolagus 3

inustus, Galaga 24

inustus, Saguinus 27

iriomatensis, Felis 80 (iriamolensis, Felis bengalensis) 80

(iriomotensis, Prionailurus) 80

iris, Amblysomus 6

(irrorata, Pithecia) 30

(inus, Macaca) 36

isabelae, Genetra genetla 75

isabellinus, Ursus arcios 70

jacchus, Callishrix 26

Jackal, Simian 67

jacksani, Bdeagale 77

jacabila, Felis 80

Jaguar 83

japonicus, Zalophus califomianus 85 javanica, Manis 44

javanica, Tupaia 19

javanicus, Bos 96

javanus, Glischropus 17

Javelina 90

jayakori, Hemitragus 100

jentinki. Cephalophus 98

jerdoni. Capra falconeri 97

jerdoni. Paradaxurus 76

johnii, Presbytis 39

(jahnii, Trachypithecus) 39

johnsioni, Geneua 76

(jubaia, Mymecophaga) 42

jubatus, Acerodon 11

jubatus, Acinonyx 78

julianae, Amblysamus 6

Kangaroo-rat, Morro Bay 48

Kangaroo-rat, Texas 48

kathiah, Mustela 74

keramae, Cervus nippon 92

Kerivoula 17

khur, Equus hemianus 88

(khur, Equus onager) 88

Khur 88

(kiang, Equus) 88

Kiang 88

(kirkii, Colobus badius) 35

kirkii, Colobus pennantii 35

(kirkii, Colobus) 35

(kirkii, Procolabus) 35

kivuana, Crocidura 8

Klipspringer, Westem 100

klossii, Hylabates 41

Kobus 100

Kodkod 80

Kogia 58

(kopschi, Cervus nippon) 92

korrigum, Damaliscus lunatus 99

koslowi. Ochalana 45

Kouprey 96

kreffiti, Lasiorhinus 3

(kuhli, Aris) 93

kuhli, Cervus porcinus 93

kuhni, Liberiictis 78

(kulan, Equus) 88

Kulatı 88

kusnotoi, Megaerops 11

labialus, Saguinus 27

Lagenadelphis 60

Lagenorhynchus 60-61

(Lagenorhynchus) 61

Lagarchestes 4

Lagostrophus 4

lagothricha. Lagothrix 30

Lagathrir 30

lagotis, Macrotis 2 
lakedownensis, Leggadina 51

Lama 91

Lamattei, Micropotamagale 6

Langur, Banded 39

Langur, Black-shanked Douc 40

Langur, Capped 40

Langur, Common 39

Langur, Douc 40

Langur, Entellus 39

Langur, Golden 39

Langur, Grey 39

Langur, Hanuman 39

Langur, John's 39

Langur, Maroon 40

Langur, Nilgiri 39

Langur, Pagai 1stand 37

Langur, Phayre'a 39

Langur, Pig-tailed 37

Langur, Red-shanked Douc 40

Langur, Silvered 38

Langur, Spectacled 39

Langur, Thomas's 40

Langur, White-faced 39

(laniger, Avahi) 23

laniger, Lichanotus 23

lanigera, Chinchilla 54

lanosa, Crocidura 8

laptevi, Odobenus rosmarus 85

lar, Hylobales 41

larvata, Paguma 76

larvatus, Nasalis 37

Lasiarhinus 3

(Lasiorhinus) 3

Lasiurus 17

Latidens 11

latona, Crocidura 8

layardii, Mesaplodon 57

leadbeateri, Gymnabelideus 3

leche, Kobus 100

Lechwe 100

Leggadina 51

Lemur 20-21

Lemur, Alaotran Gentle 20

Lemur, Black 21

Lemur, Broad-nosed Gentle 20

Lemur, Brown 21

Lemur, Collared 21

Lemur, Coquerel's Dwarf 22

Lemur, Crowned 20

Lemur, Dry-bush Weasel 22

Lemur, Fat-tailed Dwarf 22

Lemur, Fork-marked 23

Lemur, Golden 20

Lemur, Golden Bamboo 20

Lemur, Greater Bamboo 20

Lemur, Greater Dwarf 22

Lemur, Greater Weasel 22

Lemur, Grey-backed Sportive 21

Lemur, Grey Gentle 20

Lemur, Hairy-eared Dwarf 22

Lemur, Lesser Weasel 22

Lemur, Light-necked Sportive 22

Lemur, Mayotte 21

Lemur, Milne-Edwards' Sportive 21

Lemur, Mongoose 2I

Lemur, Northem Sportive 22

Lemur, Nossi-be Sportive 21

Lemur, Red-bellied 2)

Lemur, Red-fronted 21
Lemur, Red-tailed Sportive 22

Lemur, Ring-tailed 20

Lemur, Ruffed 22

Lemur, Sanford's 21

Lemur, Sclater's 21

Lemur, Sportive 21, 22

Lemur, Western Gentle 20

Lemur, White-collared 21

Lemur, White-footed Sportive 22

Lemur, White-fronted 20

Lemur, Woolly 23

(Lemur) 22

(lemurinus, Aotus) 28

(lentiginosa, Sausa) 62

lea, Panthera 83

leonina, Mirounga 85

Leantopithecus 26

Leopard, Clouded 83

Leopard, Snow 84

Lepilemur 21-22

leparides, Lagorchestes 4

Leporillus 51

leptoceros, Gazella 99

Leptonycteris 17

Lepus 45

Lervia, Ammorragus 95

lesueuri, Benangia 3

leucas, Delphinapterus 58

leucippe, Callithrix argentata 25

leucocephala, Presbytis francoisi 39

(leucocephalus, Trachypithecus) 39

(leucogenys, Hylobates) 41

leucaphaeus, Hippatragus 100

(leucophaeus, Mandrillus) 38

leucophaeus, Papio 38

leucopterus, Pteropus 13

(leucapus, Lenur mustelinus) 22

leucopus, Lepilemur 22

leucopus, Saguinus 27

(leucopus, Vulpes vulpes) 68

leucoryx, Oryx 101

leucotis, Dipodomys microps 48

leucotis, Pterapus 13

leucura, Macratis 2

Ihoesti, Cercapithecus 32

liberiensis, Choeropsis 90

liberiensis, Poiana richardsoni 76

Liberiictis 78

Lichanotus 23

lignicolar, Paradorunus hemaphroditus 76

(lignicolor, Paradaxurus) 76

limicola, Reithrodontomys megalotis 50

Limnogale 5

lineaus, Vampyrops 17

linsang. Prionodon 76

Linsang, Banded 76

Linsang, Leighton's 76

Linsang, Spotted 76

Lion 83

Lion, Asiatic 83

Lipates 56

Lissodelphis 61

littoralis, Urocyon 68

livingstonei, Pteropus 13

lombacensis, Pteropus 13

(longicaudata, Manis) 44

longicaudala, Microgale 5

longicaudata, Sminthopsis 1

longicandatus, Myasorex 9 longicaudatus, Noumys 52

longicaudis, Lutra 72

longicaudis, Lutra longicaudis 72

longipes, Crocidura 8

longipes, Potorous 4

longirostris, Micragale 5

longirostris, Stenella 63

Loris 23

Loris, Lesser Slow 24

Loris, Pygmy 24

Loris, Slender 23

Loris, Slow 24

(lowei, Cynogale) 75

Lowii, Prilocercus 19

Laxodonta 86

lucifer, Acerodon 11

lucina, Crocidura 8

ludia, Crocidura dolichura 8

(ludia, Crocidura) 8

lullulae, Phalanger 2

(lullulae, Phalanger orientalis) 2

lumhalizi, Dendralagus 4

lunata, Onychogalea 4

lunatus, Damaliscus 99

lupus, Canis 66

(luteus, Equus) 88

lukeus, Zapus hudsonius 53

(luteus, Zapus princeps) 53

lutra, Lutra 72

lutra, Lutra lutra 72

Lutra 72-73

(Lutra) 72

lutreola, Mustela 74

lutrealina, Misstela 74

lutris, Enhydra 71

(lybica, Felis) 82

Lycaon 68

lylei, Pleropus 13

lynx, Felis 81

(lynx, Lynx) 81

Lynx, Bay 82

Lynx, Eurasian 81

Lynx, Pardel 81

Lynx, Spanish 81

(Lynx) 79-82

Macaca 36-37

(Macaca) 37

macaco, Lemur macaca 2)

Macaque, Assam 36

Macaque, Barbary 37

Macaque, Bear 36

Macaque, Bonnet 37

Macaque, Booted 37

Macaque, Celebes Black 37

Macaque, Crab-eating 36

Macaque, Formosan Rock 36

Macaque, Gorontalo 37

Macaque, Heck's 37

Macaque, Japanese 36

Macaque, Lion-tailed 37

Macaque, Long-tailed 36

Macaque, Mentawai 36

Macaque, Moor 36

Macaque, Muna-Butung 37

Macaque, Père David's 37

Macaque, Pigtail 36

Macaque, Rhesus 36

Macaque, Stump-tsiled 36

Macaque, Sulawesi 37 
Macaque, Taiwan 36

Macaque, Tibetan 37

Macaque, Tonkean 37

Macaque, Toque 37

Macaque, Yakushima 36

macklori, Acerodon 11

macleayi, Dorcopsis 4

(macleayi, Dorcopsulus) 4

(macneilli, Cervus canadensis) 92

macneilli, Cervus elaphus 92

macrocephalus, Physeter 58

Macroderma 16

Macrogalidia 76

Macropus 4

macrorhynchus, Globicephala 60

macrotis, Idiunus 49

macrotis, Notomys 52

macrotis, Pleropus 13

Macrotis 2

macroura, Ratufa 47

Macruromys 51

maculatus, Phalanger 2

(maculatus, Spilocuscus) 2

(maculicollis, Hydrictis) 73

maculicallis, Lutra 73

madagascariensis, Daubentonia 23

mahaganus, Pteropus 13

major, Cheirogaleus 22

(major, Eupleres) 75

major, Phyllonycteris 17

majori, Microgale 5

(malayana, Stenella) 62

malayanus, Helarctos 69

Manatee, African 87

Manatee, Amazonian 87

Manatee, American 87

Manatee, Caribbean 87

Manatee, North American 87

Manatee, South American 87

manatus, Trichechus 87

mandarinus, Cervus nippon 92

Mandrill 38

(Mandrillus) 38

manengubae, Crocidura 8

Mangabey, Agile 31

Mangabey, Black 31

Mangabey, Collared 31

Mangabey, Grey-cheeked 31

Mangabey, Red-capped 31

Mangabey, Sanje 31

Mangabey, Sooty 31

Mangabey, Tana River 31

Mangabey, White-cheeked 31

Mangabey, White-collared 31

Manis 44

manul, Felis 81

maquassiensis, Crocidura 8

margarita, Felis 81

Margay 83

marginata, Caperea 66

mariannus, Pteropus 13

(marica, Gazella lepiaceras) 100

marica, Gazella subguturosa 100

(maritimus, Thalarctos) 70

maritimus, Ursus 70

Markhor 97

Markhor, Kabul 97

Markhor, Straight-horned 97

Marl 2 marmorala, Felis 81

Marmoset, Black-tailed 25

Marmoset, Buffy-headed 26

Marmoset, Buffy-tufted-ear 26

Marmoset, Common 26

Marmoset, Goeldi's 27

Marmoset, Pygmy 26

Marmoset, Silvery 25

Marmoset, Tassel-eared 26

Marmoset, White 25

Marmoset, White-eared 26

Marmol, Menzbier's 46

Marmot, Vancouver lsland 47

Marmola 46-47

Marshbuck 102

Marten, Nilgiri 73

Marten, Taiwan Yellow-throated 73

Marten, Tsushima Island 73

Martes 73

martinensis, Neotoma 50

(martinsi, Saguinus) 26

maura, Macaca 36

maxima, Paracrocidura 10

maximus, Elephas 86

maximus, Priodonies 43

mayensis, Odocoileus virginianus 94

mayotiensis, Lemur fulvus 21

Mazama 93

meamsi, Pheropus 13

(meamsi, Pteropus hypomelanus) 13

medius, Cheirogaleus 22

megaceros, Capra falconeri 97

Megaerops 11

megalotis, Darcatragus 99

(megaloris, Notomys) 52

Megapiera 65

megaspila, Viverra 76

melaena, Globicephala 60

melalophos, Presbytis 39

melanocephalus, Cacajao 29

melanoleuca, Ailuropoda 69

melanopagan, Preropus 14

melanorrhachis, Microgale 5

melanous, Pleropus 14

melanura, Dendrogale 19

melanurus, Capromys 55

Mellivora 73

Melogale 74

Melomys 52

Melomys, While-toothed 52

Melursus 69

menzbieri, Marmola 46

mephistophiles, Pudu 95

mergulus, Limnagale $S$

meridionalis, Capromys 55

(meridionalis, Mysateles) 55

Merrin 4

Mesoplodon 57-58

(Mesoplodon) 56

(mesopotamica, Dama) 91

mesopotamicus, Cervus dama 91

(mexicana, Tamandu) 42

mexicanus, Castar canadensis 49

(mexicanus, Caendou) 54

mexicanus, Cynomys 46

mexicanus, Sphiggurus 54

michaelis, Felis caracal 80

(michaelis, Lynx caracal) 80

(miconax, Aotus) 28
Microcebus 22-23

(microdon, Aonyx) 7

(microdon, Lemur mustelinus) 22

microdon, Lepilemur 22

Micragale 5-6

Micropolamogale 6

(microps, Stenella) 63

microtis, Dusicyon 67

Microtus 51

midas, Saguinus 27

(mindorensis, Anoa) 97

mindorensis, Bubalus 97

minor, Dabsania 11

minor, Platanista 56

(minor, Platanista gangetica) 56

minor, Tupaia 19

(Miopithecus) 33

Mirounga 85

minus, Mesoplodon 57

(Mirza) 22

miris, Cercopithecus 33

(modesius, Callicebus) 29

(moholi, Galaga) 25

Mole, De Winton's Golden 7

Mole, Duthie's Golden 7

Mole, Giant Golden 7

Mole, Grants' Golden 7

Mole, Gunning's Golden 6

Mole, Juliana's Golden 6

Mole, Rough-haired Golden 7

Mole, Sclater's Golden 7

Mole, Somali Golden 7

Mole, Van Zyl's Golden 7

Mole, Visagie's Golden 7

Mole, Yellow Golden 7

Mole, Zulu Golden 6

moloch, Callicebus 29

moloch, Hylobates 41

(malach, Hylobates lar) 41

molassinus, Pteropus 14

mona, Cercopithecus 33

monachus, Momachus 85

monachus, Pithecia 30

Monachus 85-86

manax, Cracidura 9

Mongoose, Brosd-striped 77

Mongoose, Brown-tailed 78

Mongoose, Giant-striped 77

Mongoose, Jackson's 77

Mongoose, Liberian 78

Mongoose, Narrow-striped 78

Mongoose, Small Indian 77

Mongoose, Sokoke Bushy-tailed 77

mongoz, Lemur 21

Monkey, Allen's Swamp 31

Monkey, Banded Leaf 39

Monkey, Black-browed Spider 28

Monkey, Black-cheeked While-nosed 32

Monkey, Black-handed Spider 28

Monkey, Black Leaf 39

Monkey, Black Spider 29

Monkey, Blue 33

Monkey, Brelich's Snub-nosed 40

Monkey, Brown-headed Spider 28

Monkey, Campbell's 32

Monkey, Capped Leaf 40

Monkey, Central American Squirrel 30

Monkey, Common Squirrel 30

Monkey, Common Wcolly 30 
Monkey, Crowned 33

Monkey, Cynomolgus 36

Monkey, de Brazza's 33

Monkey, Diademed 33

Monkey, Diane 32

Monkey, Dryas 32

Monkey, Dusky Leaf 39

Monkey, Ebony Leaf 38

Monkey, Geoffroy's Spider 28

Monkey, Golden-bellied 33

Monkey, Golden Leaf 39

Monkey, Golden Snub-nosed 40

Monkey, Greater White-nosed 33

Monkey, Green 31

Monkey, Grey Leaf 39

Monkey, Grey Snub-nosed 40

Monkey, Grivet 31

Monkey, Guizhou Snub-nosed 40

Monkey, Hose's Leaf 39

Monkey, Humboldt's Woolly 30

Monkey, Java Leaf 38

Monkey, L'Hoest's 32

Monkey, Lesser White-nosed 33

Monkey, Long-haired Spider 28

Monkey, Long-nosed 37

Monkey, Maroon Leaf 40

Monkey, Mitred Leaf 39

Monkey, Mona 33

Monkey, Mountain 32

Monkey, Moustached 32

Monkey, Night 28

Monkey, Owl 28

Monkey, Owl-faced 32

Monkey, Patas 35

Monkey, Phayre's Leaf 39

Monkey, Preuss's 32

Monkey, Proboscis 37

Monkey, Purple-faced Leaf $\mathbf{4 0}$

Monkey, Putty-nosed 33

Monkey, Red-backed Squirrel 30

Monkey, Red-bellied 32

Monkey, Red-eared 32

Monkey, Red Spider 28

Monkey, Redtail 32

Monkey, Rhesus 36

Monkey, Savanna 31

Monkey, Sichuan Snub-nosed 40

Monkey, Silvered Leaf 38

Monkey, Spectacled Leaf 39

Monkey, Spot-nosed 33

Monkey, Sun-tailed 33

Monkey, Sykes' 33

Monkey, Tantalus 31

Monkey, Thomas's Leaf 40

Monkey, Tonkin Leaf 39

Monkey, Tonkin Snub-nosed 40

Monkey, Vervet 31

Monkey, White-bellied Spider 28

Monkey, White-fronted Leaf 39

Monkey, White-headed Black Leaf 39

Monkey, Wolfs 33

Monkey, Woolly Spider 29

Monkey, Yellow-tailed Woolly 30

Monkey, Zaire Diana 33

monoceros, Monodon 59

Monodon 59

monrana, Tupaia 19

montana, Vulpes vulpes 68

monticola, Cephalophus 98 (monticola, Philaniombo) 98

monticularis, Bunolagus 45

Moonrat, Mindanao 7

mordax, Notomys 52

morroensis, Dipodomys heermanni 48

moschata, Desmana 10

moschifenus, Moschus 94

(moschifenus, Moschus moschifenus) 94

moschus, Moschus 94

Moschus 94

(Moschus) 94

Mouflon, Cyprus 101

Mouflon, Mediterranean 101

Mouse, Alabama Beach 50

Mouse, Alice Springs 52

Mouse, Anastasia Island Beach 50

Mouse, Choctawhatchee Beach 50

Mouse, Gould'a 52

Mouse, Hastings River $\mathbf{5 2}$

Mouse, Key Largo Cotton 50

Mouse, Lakeland Downs 51

Mouse-lemur, Brown 23

Mouse-lemur, Coquerel's 22

Mouse-lemur, Fork-marked 23

Mouse-lemur, Grey 22

Mouse-lemur, Lesser 22

Mouse-lemur, Rufous 23

Mouse-lemur, Russet 23

Mouse, Los Angeles Little Pocket 48

Mouse, Perdido Key Beach 50

Mouse, Pilliga 52

Mouse, Point Reyes Jumping 53

Mouse, Salinas Pocket 48

Mouse, Salimarsh Harvest 50

Mouse, Shark Bay 52

Mouse, Shortridge's Native 53

Mouse, Smoky 52

Mouse, South-eastern Beach 50

Mouse, Southern Marsh Harvest 50

Mouse, Western 52

Mouse, White-eared Pocket 48

muelleri, Hylobates 41

(muelleri, Hylobates lor) 41

muenninki, Tokudaia osimensis 53

mulatia, Macaca 36

muliensis, Ochotona 45

Mungotictis 78

Muntiacus 94

Muntjac, Black 94

Muntjac, Fea's 94

murina, Dendrogale 19

murinus, Microcebus 22

Muriqui 29

Musang 76

musculus, Balaenoptera 65

musimon, Ovis 101

(musimon, Ovis ammon) 101

(musimon, Ovis orientalis) 101

musschenbroekii, Mocrogalidio 76

Musiela 74

mustelinus, Lepilemur 22

mutus, Bos 96

myoides, Xeromys 53

Myosorex

myotis, Myotis 18

Myoris 17-18

Myotis, Bartel's 18

Myotis, Grey 18

Myotis, Herman's 18
Myrmecobius 1

Myrmecophaga 42

(Mysateles) 55

Mystacina 18

mystax, Saguinus 27

mysticetus, Balaena 66

Myzopoda 17

(nancymai, Aotus) 28

nanus, Capromys 55

(narica, Nasua) 70

Narwhal 59

Nasalis 37

nasomaculatus, Addax 95

nasua, Nasua 70

Nasua 70

(Nasua) 70

novaho, Microtus mexicanus 51

nebulosa, Neofelis 83

neglectus, Cercopithecus 33

nelsoni, Nasua 70

nelsoni, Oryzomys 50

nelsoni, Ursus arctos 70

nemaeus, Pygathrix 40

nemestrina, Macaca 36

Nemorhaedus 100

(Nemorhaedus) 100

Neofelis 83

neohibernicus, Pleropus 14

Neophocaena 63

Neoroma 49

nereis, Enhydra lutris 72

Nesolagus 45

netscheri, Nesologus 45

nicobarica, Tupaia 19

nictilans, Cercopithecus 33

(niger, Canis) 67

niger, Pleropus 14

nigra, Aplodontia rufa 46

nigra, Macoca 37

(nigrescens, Macaca) 37

(nigriceps, Aotus) 28

nigricollis, Saguinus 27

nigripes, Felis 81

nigripes, Mustela 74

nigripes, Pygathrix nemaeus 40

(nigrivittotus, Cebus) 29

nigroviridis, Allenopithecus 31

(nigroviridis, Cercopithecus) 31

nimbae, Crocidura 9

nitendiensis, Pteropus 14

nitratoides, Dipodomys nitratoides 48

nivalis, Leptonycteris 17

niveiventris, Peromyscus polionotus 50

notatus, Dendrologus dorianus 3

Notomys 52

(Notomys) 52

novaeangliae, Megaptera 65

(Novibos) 96

Numbat 1

(nuuanu, Tursiops) 63

Nycticebus 24

Nyctimene 12

oaxacensis, Glaucomys volans 46

obliquidens, Lagenorhynchus 61

obscura, Presbytis 39

ohscurus, Lagenorhynchus 61

(obscurus, Trachypithecus) 39

obtissirostris, Calcochloris 7

(obtusirostris, Chlorotolpa) 7 
occidenialis, Hapalemur griseus 20 occidentalis, Microgale 6 occidentalis, Pseudomys 52 (occulta, Feresa) 59

Ocelot 81

Ocelot, Tree 83

Ochotona 45

ochreata, Macaca 37

Octamys 55

ocularis, Pteropus 14

Odobenus 85

Odocoileus 94

(Odocoileus) 95

oedipus, Saguinus 27

oedipus, Saguinus oedipus 27

(oenanthe, Callicebus) 29

oerstedii, Saimiri 30

ogilbyi, Cephalophus 98

oi, Sus barbatus 90

(olallae, Callicebus) 29

Olingo, Bushy-tailed 70

olivaceus, Cebus 29

olulla, Sylvisorex 10

omnivora, Bdeogale crassicauda 77

(onager, Equus) 88

onca, Panthera 83

Oncilla 82

Onychogalea 4

(ophion, Ovis aries) 101

ophion, Ovis orientalis 101

oralis, Pseudomys 52

Orang-utan 42

orarius, Zapus rinolatus 53

orca, Orcinus 61

Orca 61

Orcaella 61

Orcinus 61

(Orcinus) 61

Oreotragus 100

orientalis, Geogale aurita 5

orientalis, Melogale personata 74

(orientalis, Melogale) 74

orientalis, Phalanger 2

(omata, Felis) 82

ornata, Rupicapra rupicapra 102

ornatus, Pteropus 14

omatus, Tremarctos 69

Oryx 101

Oryx, Arabian 101

Oryx, Scimitar-homed 101

Onyzomys 50

Otolemur 25

(Otolemur) 25

Onomops 18

Otopteropus 12

Otter, African Clawless 71

Otter, Cameroon Clawless 71

Otter, Cape Clawless 71

Otter-civet 75

Otter, Eurasian 72

Otter, European 72

Otter, Giant 74

Otter, Giant Brazilian 74

Otter, Hairy-nosed 73

Otter, La Plata 72

Oner, Marine 72

Oner, Neotropical River 72

Otter, North American 72

Oter, Oriental Small-clawed 71
Otter, Sea 71

Otter-shrew, Nimba 6

Otter-shrew, Ruwenzori 6

Otter, Smooth-coated 73

Otter, South American River 72

Otter, Southem River 73

Otter, Southem Sea 72

Otter, Speckle-throated 73

Otter, Spot-necked 73

Otter, Zaire Clawless 71

Otter-shrew, Nimba 6

Otter-shrew, Ruwenzori 6

Ounce 84

(oustaleri, Colobus badius) 35

ousualeri, Colobus pennantii 35

(oustaleti, Procolobus nufomilratus) 35

Oris 101

owstoni, Chrotogale 75

Ozotoceros 94

paca, Agouti 54

(paca, Cuniculus) 54

pacificus, Indopacetus 56

(pacificus, Mesoplodon) 56

pagensis, Macaca nemestrina 36

(pagensis, Macaco) 36

Paguma 76

(pajeros, Felis) 80

palawanensis, Tupaia 19

(palawanensis, Tupaia glis) 19

palliata, Alouatta 28

pallida, Vulpes 68

Pan 12

panamensis, Ateles geoffroyi 28

Panda, Giant 69

Panda, Lesser 70

Panda, Red 70

Pangolin, Black-bellied 44

Pangolin, Cape 44

Pangolin, Chinese 44

Pangolin, Giant 44

Pangolin, Giant Ground 44

Pangolin, Indian 44

Pangolin, Long-tailed 44

Pangolin, Malayan 44

Pangolin, Temminck's Ground 44

Pangolin, Three-cusped 44

Pangolin, Tree 44

Pangolin, White-bellied 44

paniscus, Ateles 29

paniscus, Pan 42

Panthera 83-84

Pantholaps 101

papio, Popio hamadryas 38

(papio, Papio) 38

Papio 38

(Papio) 38

papua, Hipposideros 16

Paracrocidura 9-10

Paradoxurus 76

paradoxus, Solenodon 5

(Parantechinus) I

pardalis, Felis 81

pardicolor, Prionodon 76

pardina, Felis 81

(pardina, Felis lynx) 81

(pardinus, Lynx) 81

pardus, Panthera 83

parvidens, Cynomys 46

parvula, Microgale 6 parvus, Burramys 3

(palas, Cercopithecus) 35

palas, Erythrocebus 35

paucidentata, Alionycteris II

pecari, Toyassu 90

Peccary, Chacoan 90

Peccary, White-lipped 90

pedunculatus, Zyzomys 53

peli, Anomalurus 49

pembertoni, Peromyscus 50

penicillata, Bettongia 3

peninsularis, Antilocapra americana 95

pennannii, Colobus 34

(pennanii, Colobus badius) 34

(pennantii, Procolobus) 34

pentadacryla, Manis 44

Pentalagus 45

Peponocephala 61

Perameles 2

peregusna, Vomela peregusna 75

permixtio. Phalanger 2

Perodicticus 24

Perognathus 48

Peromyscus 50

peronii, Lissodelphis 61

persephone, Petrogale 4

persica, Panthera leo 83

personotus, Callicebus 29

personolus, Pheropus 14

perspicillata. Lura 73

petaurista, Cercopithecus 33

petersi, Aepyceros melampiss 95

petersi, Rhynchocyon 10

Petrodromus 10

Petrogale 4

(Pettenus) 20

phaea, Aplodontia rufo 46

phaeocephalus, Pteropus 14

phaeura, Crocidura 9

Phalanger 2

Phalanger, Common 2

Phalanger, Spotted 2

Phaner 23

Phascogale 1

Phascogale, Red-tailed I

phasma, Peromyscus polionotus 50

(Phataginus) 44

phoyrei, Presbytis 39

(phoyrei, Trachypithecus) 39

(Philantomba) 98

philippii, Arctocephalus 84

Phoca 86

phocaenoides, Neophocaena 63

phocoena, Phocoena 63

Phocoena 63-64

(Phocoena) 63

Phocoenoides 64

Phyllonycteris 17

physalus, Balaenoptera 65

Physeter 58

victa, Tupaia 20

pictus, Lycaon 68

Pig. Javan Warty 90

Pig, Ryukyu Islands Wild 90

Pig, Viss yan Warty 90

Pig, Western Bearded 90

pigra, Alowana 28

Pika, Kozlov's 45

Pika, Muli 45 
pileata, Presbytis 40

pileatus, Hylobates 41

(pileatus, Hylobates lar) 41

(pilearus, Saguinus) 27

(pileaus, Trachypithecus) 40

pilligaensis, Pseudomys $\$ 2$

pilosus, Preropus 14

pinchaque, Tapinus 89

pithecia, Pithecia 30

Pithecia 30

(Pilhecia) 30

Pitymys 51

(plagiodon, Stenella) 62

(Plagiodonia) 55

Plagiodantia 55

planiceps, Felis 81

planifrons, Hyperoodon 56

Platanista 56

(platensis, Lutra) 72

platyops, Potorous 4

Plecous 18

plenus, Sigmodan arizonae 50

(plenus, Sigmodon hispidus) 50

(plumbea, Sausa) 62

Podogymnura 7

poeyi, Phyllonycteris 17

poganias, Cercopithecus 33

pohlei, Pteropus 14

(pohlei, Pzerapus macrotis) 14

Poiana 76

palia, Crocidura dolichura 8

(polia, Crocidura) 8

poliocephalus, Pteropus 14

palli, Myasorex 9

palykomas, Colobus 35

ponceleri, Solomys 53

Pongo 42

Pontoporia 56

Porcupine, North African Crested 53

Porcupine, Spiny Tree 54

Porcupine, Thin-spined 53

Porpoise, Black 64

Porpoise, Burmeister's 64

Porpoise, Common 63

Porpoise, Dall's 64

Porpoise, Finless 63

Porpoise, Gulf 64

Porpoise, Harbour 63

Porpoise, Spectacled 63

Porpoise, White-flanked 64

porteousi, Oreotragus areotragus 100

Possum, Leadbeater's 3

potenziani, Presbytis 40

Potoroo, Broad-faced 4

Potono, Long-footed 4

Potarous 4

Polos 70

potto, Peradicticus 24

Potto 24

Potto, Golden 23

praecanis, Pseudomys 52

Presbyris 38-40

(Presbytis) 39

preussi, Cercopithecus thaesti 32

(preussi, Cercopithecus) 32

preussi, Colobus 35

(preussi, Colabus badius) 35

(preussi, Procolobus pennani) 35

principula, Microgale 6
Priodontes 43

(Prianailurus) 80

Prionodon 76

(Procolobus) 34-35

Procyon 71

Prolagus 45

prolixicaudata, Microgale 6

Pronghom, Baja California 95

Pronghom, Mexican 95

Propithecus 23

Proteles 78

provocax, Lutra 73

przewalskii, Equus 88

(przewalskii, Equus caballus) 88

(przewalskii, Equus ferus) 88

psammophila, Sminthopsis 1

psammophilus, Perognathus inamatus 48

(psammophilus, P. Longimembris) 48

pselaphon, Pleropus 14

pseudaxis, Cervus nippon 92

Pseudomys 52-53

Pseudorca 61

Pteranura 74

Pteropus 12-16

(Pteropus) 11

Ptilocercus 19

Pucu, Guasu 91

pudu, Pudu 95

Pudu 95

pulla, Microgale 6

Puma 80

Puma, Costa Rican 80

Puma, Eastern 80

Puma, Florida 80

pumilus, Pteropus 14

pumilus, Tarsius spectrum 25

(pumilus, Tarsius) 25

punctala, Dasyprocta 54

(Pusa) 86

pusilla, Vulpes vulpes 68

pusillus, Arctocephalus 85

Pygathrix 40

(pygerythrus, Cercopithecus) 31

pygmaea, Cebuella 26

pygmaeus, Nycricebus 24

pygmaeus, Ponga 42

pygmaeus, Procyon 71

Pygmy-possum, Mountain 3

pyrenaica, Capra pyrenaica 97

pyrenaicus, Galemys 10

(pyrthus, Presbytis) 38

quadricornis, Tetracenus 102

quagga, Equus 88

Quagga 88

(quarlesi, Anoa) 97

quarlesi, Bubalus 97

Rabbit, Assam 45

Rabbit, Lower Keys Marsh 45

Rabbit, Riverine 45

Rabbit, Ryukyu 45

Rabbit, Sumatran 45

Rabbit, Sumatran Shon-eared $\mathbf{4 5}$

Rabbit, Tehuantepec Jack 45

Rabbit, Volcano 45

rabori, Nyctimene 12

Raccoon, Barbados 71

Raccoon, Cozumel Island 71

radiata, Macaca 37

raffrayana, Emballonura 16 raineyi, Crocidura luna 8

(raineyi, Crocidura) 8

Rat, Blunt-faced 53

Rat, Colorado River Cotton 50

Rat, False Swamp 53

Rat, Fresno Kangaroo 48

Rat, Giant Kangaroo 48

Rat, Giluwe 53

Rat, Greater Stick-nest 51

Rat, Heath 53

Rat, Houserock Chisel-toothed Kanga roo 48

Rat-kanganoo, Desert 3

Rat-kangaroo, Lesuewr's 3

Rat, Lesser Small-toothed 51

Rat, Lesser Stick-nest 51

Rat, Luzon Forest 51

Rat, Nelson's Rice 50

Rat, North Okinawa Spiny 53

Rat, Red Vizcacha 55

Rat, Rock-dwelling 53

Rat, San Quintin Kangaroo 48

Rat, Silver Rice 50

Rat, Stephens' Kangaroo 48

Rat, Tipton Kangaroo 48

Rat, Western Small-toothed 51

Rat, White-tipped Stick-nest 51

Ratel 73

Ratuus 53

Ratufa 47

raviventris, Reithrodontomys 50

rayneri, Pteropus 15

Redunca 101

Reedbuck, Westem Mountain 101

Reithrodantamys 50

remyi, Suncus 10

(retusa, Bumeisteria) 43

retusus, Chlamyphonus 43

Rhim 99

Rhinoceros 89

Rhinoceros, Black 89

Rhinoceros, Great Indian 89

Rhinoceros, Hook-lipped 89

Rhinoceros, Javan 89

Rhinoceros, Northern Square-lipped 89

Rhinoceros, Northern White 89

Rhinoceros, Southern Square-lipped 89

Rhinoceros, Southem White 89

Rhinoceros, Sumatran 89

Rhinonicteris 17

(Rhinopithecus) 40

Rhynchacyon 10

ridleyi, Hippasideras 16

riparia, Neotama fuscipes 49

riukiuanus, Sus scrofa 90

robusta, Mystacina 18

rabustus, Eschrichtius 64

Rock-rat, Central 53

Rock-rat, Macdonnell Range $\mathbf{5 3}$

Rock-wallaby, Prosperine 4

rodricensis, Pleropus 15

(roloway, Cercoptihecus) 32

Romerolagus 45

Rorqual, Common 65

rosalia, Leantopithecus rosalia 26

(rosalia, Leantopithecus) 26

(roseiventris, Stenella) 63

rosmarus, Odobenus 85

(rostratus, Hyperoodon) 56

(roulini, Tapinus) 89 
roxellana, Pygathrix 40 (roxellanae, Rhinopithecus) 40

rubicunda, Presbytis 40

(rubicundus, Cacajao) 29

rubidus, Cephalophus nigrifrons 98

rubiginasa, Felis 82

rubriventer, Lemur 21

nueppelli, Vulpes 68

nufa, Felis 82

(nuficaudatus, Lemur mustelinus) 22

ruficaudatus, Lepilemur 22

rufifrons, Gazella 100

nfomitratus, Colobus 35

(nufomitratus, Colobus badius) 35

(nifomitratus, Procalobus nufomitratus) 35

rufoniger. Phalanger 2

(nufoniger, Spilocuscus) 2

rufus, Canis 67

nufus, Lemur fulvus 21

(rufius, Lemur macaca) 21

(rufus, Lynr) 82

nufus, Microcebus 23

(rufus, Microcebus murinus) 23

rufus, Pteropus 15

Rupicapra 101-102

ruwenzonii, Micropotamogale 6

(Ruwenzarisorex) 10

(sabaeus, Cercopithecus) 31

Saguinus 26-27

(Saguinus) 26-27

saimensis, Phoca hispida 86

(saimensis, Pusa hispida) 86

Saimiri 30

(Saimiri) 30

Saki, Bearded 30

Saki, Black 30

Saki, Black-and-white 30

Saki, Buffy 30

Sakj, Monk 30

Saki, Pale-headed 30

Saki, Red-bearded 30

Saki, Red-nosed 30

Saki, Shaggy 30

Saki, Southem Bearded 30

Saki, White-faced 30

Saki, White-nosed 30

Salano 78

Salanoia 78

salimalii, Latidens 11

salongo, Cercopithecus 33

salvanius, Sus 90

samoensis, Pteropus 15

(sanbomi, Leptonycteris) 17

sanfelipensis, Capromys 55

(sanfordi, Lemur macaca) 21

Sangai 92

sangi, Petrodromus tetradactylus 10

'sanjei', Cercocebus galeritus 31

santacrucis, Preropus 15

(santacrucis, Plerapus hypamelanus) 15

sardus, Prolagus 45

satanas, Chiropates 30

satanas, Chiropotes salanas 30

satanas, Colobus 35

(sauveli, Novibos) 96

scapulatus, Pleropus 15

schalleri, Myosorex 9

schauinslandi, Monachus 86

scheffeli, Felis margarila 81 scirpensis, Microtus californicus 51 sciureus, Saimiri 30

Sciurus 47

scloteri, Cercopithecus erythrotis 32

sclateri, Chlorotalpa 7

Seal, Afro-Australian Fur $\mathbf{8 5}$

Seal, Amsterdam Island Fur 85

Seal, Antarctic Fur 84

Seal, Cape Fur 85

Seal, Caribbean Monk 86

Seal, Galapagos lslands Fur 84

Seal, Guadalupe Fur 85

Seal, Hawaiian Monk 86

Seal, Juan Femandez Fur 84

Sesl, Kerguelen Fur 84

Seal, Kuril 86

Seal, Mediterranean Monk 85

Seal, New Zealand Fur 84

Seal, Saimaa Ringed 86

Seal, South African Fur 85

Seal, South American Fur 84

Seal, Southern Fur 84

Seal, Sub-antarctic Fur 85

Seal, West Indian 86

Sealion, Japanese 85

sechurae, Dusicyon 67

Selenarctos 69

selina. Crocidura luna 8

(selina, Crocidura) 8

(Semnapithecus) 39

senegalensis, Galago 25

senegalensis, Trichechus 87

senex, Presbytis 40

seniculus, Alouatta 28

(septentrionalis, Lemur mustelinus) 22

septentrionalis, Lepilemur 22

Serow, Formosan 97

Serow, Mainland 97

Serow, Sumatran 98

serval, Felis 82

Serval 82

seychellensis, Coleura 16

seychellensis, Pheropus 15

shawmayeri, Dendrolagus goodfellawi 3

(shawmayeri, Dendrolagus matschiel) 3

Sheep, Barbary 95

shepherdi, Tasmacetus 58

shoritidgei, Pseudomys 53

Shou 92

Shrew, Gabon Dwarf 10

Shrew, Long-tailed Forest 9

Shrew, Makwassie Musk 8

Shrew-tenrec, Dark Pygmy 6

Shrew-lenrec, Drouhard's 5

Shrew-tenrec, Dusky 6

Shrew-lenrec, Gracile 5

Shrew-tenrec, Greater Long-tailed 6

Shrew-tenrec, Large-footed 5

Shrew-tenrec, Lesser Long-tailed 5

Shrew-tenrec, Long-tailed 6

Shrew-tenrec, Long-nosed 5

Shrew-tenrec, Major's Lesser Long-tailed 5

Shrew-lenrec, Pygmy 6

Shrew-tenrec, Short-tailed 5

Shrew-tenrec, Striped 5

Shrew-lenrec, Taivi 6

Shrew-tenrec, Thomas's 6

Shrew-tenrec, Western Shont-tailed 6

Shrew-tenrec, Dark Pygmy 6
Shrew-tenrec, Drouhard's 5

Shrew-tenrec, Dusky 6

Shrew-tenrec, Gracile 5

Shrew-tenrec, Greater Long-tailed 6

Shrew-tenrec, Large-footed 5

Shrew-tenrec, Lesser Long-tailed 5

Shrew-tenrec, Long-nosed 5

Shrew-tenrec, Long-tailed 6

Shrew-tenrec, Major's Lesser Long-tailed 5

Shrew-tenrec, Northern Lesser Longtailed 6

Shrew-tenrec, Pygmy 6

Shrew-tenrec, Short-tailed 5

Shrew-tenrec, Striped 5

Shrew-tenrec, Taivi 6

Shrew-tenrec, Western Short-tailed 6

Siamang 41

siamensis, Cervus eldi 92

sibirica, Mustela 74

(sibiricus, Moschus) 94

sichanicus, Cervis nippon 93

(sieboldii, Globicephala) 60

Sifaka. Diademed 23

Sifaka, Golden-crowned 23

Sifaka, Tattersall's 23

Sifaka, Verreaux's 23

Sigmodon 50

Sika, Formosan 93

Sika, North China 92

Sika, Ryukyu 92

Sika, Shansi 92

Sika, Sichuan 93

Sika, South China 92

silenus, Macaca 37

silvestris, Felis $\mathbf{8 2}$

simensis, Canis 67

(Simias) 37

simum, Ceratotherium simum 89

simus, Hapalemur 20

simus, Kogia 58

sinica, Macaca 37

sinus, Phocoena 64

Sitatunga 102

Skunk, Big Thicket Hog-nosed 71

Skunk, Patagonian Hog-nosed 71

Sloth, Brown-throated 43

Sloth, Hoffmann's Two-toed 43

Sloth, Maned 43

smalli. Neotoma faridana 49

Sminthopsis 1

Sminthopsis, Long-tailed I

smithii, Herpestes 77

sodalis, Myotis 18

soemmerringi, Gazella 100

solatus. Cercopithecus 33

Solenodon 5

Solenodon, Cuban 5

Solenodon, Haitian 5

Solenodon, Hispaniolan 5

solitaria, Nasua nusua 70

Solomys 53

sondaicus, Rhinoceros 89

sonoriensis, Antilocapra americana 95

sarella, Microgale 6

Sotalia 62

(Sotalia) 62

Sousa 62

(Sousa) 62

spadiceus, Dyacopserus II

spadix, Cephalophus 98 
(speciosa, Macaca) 36 speciosus, Pieropus 15 spectrum, Tarsius 25 spekei, Gazella 100 spekei, Tragelophus 102 Speothos 68 Sphiggurus 54 (sphinx, Mandrillus) 38 sphinx, Papio 38

(Spilocuscus) 2 spinipinnis, Phocoena 64 (spinasus, Coendou) 54 spinosus, Sphiggurus 54 splendidula, Tupaia 20 Squirrel, Beecroft's Flying 49 Squirrel, Black Giant 47 Squirrel, Bryant's Fox 47 Squirrel, Carolins Flying 46 Squirrel, Cream-coloured Giant 47 Squirrel, Delmarva Fox 47 Squirrel, Deppe's 47 Squirrel, Ebian's Palm 46 Squirrel, Grizzled Giant 47 Squirrel, Guerrero Flying 46 Squirrel, Indian Giant 47 Squirrel, Long-eared Flying 49 Squirrel, Lord Derby's Flying 49 Squirrel, Mexican Flying 46 Squirrel, Oaxaca Flying 46 Squirrel, Pel's Flying 49 Squirrel, Temminck's Giant 46 Squirrel, Virginia Flying 46 Stag, Barbary 92 Stag, Kashmir 92 stejnegeri, Mesoplodon 58 stejnegeri, Phoca vitulina 86 Stenello 62-63

(Stenella) 62-63

Sieno 63

stenocephalo, Crocidura 9 (stenocephla, Crociduro littoralis) 9 stephensi, Dipodomys 48

Stoat 74

(styx, Stenella) 62

subniger, Preropus 15

subspinosus, Chaetamys 53

sumatraensis, Capricomis 97

sumatraensis, Capricomis sumatraensis 98 sumatrana, Lutro 73

sumatrensis, Dicerorhinus 89 (sumotrensis, Didemocenus) 89 sumichrosti, Bassariscus 70 (suncoides, Ruwenzorisorex) 10 suncoides, Sylvisorex 10

Suncus 10

(superciliosus, Lagenorhynchus) 61 Sus 90

Susu 56

Susu, Ganges 56

swaynei, Alcelaphus buselaphus 95 swinhoei, Copricomis crispus 97

sylvanus, Macaca 37

sylvicultor, Cephalophus 98

Sylvilagus 45

Sylvisorex 10

(Symphalangus) 41

syndactylus, Hylabates 41

(syndactylus, Symphalangus) 41

Syntheosciurus 47 syrichta, Torsius 25

tablasi, Pieropus 15

(uablasi, Pueropus pumilus) 15

Tagua 90

Tahr, Arabian 100

Tahr, Nilgiri 100

taiouanus, Cervus nippon 93

taivo. Microgale 6

tajacu, Tayessu 90

Takh 88

Takin 97

Takin, Golden 97

Takin, Sichuan 97

talapoin, Cercopizhecus 33

(talopoin, Miopithecus) 33

Talapoin 33

(Tamandu) 42

Tamandua 42

Tamandua 42

Tamaraw 97

(tomarin, Saguinus) 27

Tamarin. Bare-faced 26

Tamarin, Black-and-red 27

Tamarin, Black Lion 26

Tamarin, Black-mantled 27

Tamarin, Brown-headed 27

Tamarin, Cotton-headed 27

Tamarin, Cotton-top 27

Tamarin, Dusky 27

Tamarin, Emperor 27

Tamarin, Geoffroy's 27

Tamarin, Golden-headed Lion 26

Tamarin, Golden Lion 26

Tamarin, Golden-rumped Lion 26

Tamarin, Mottle-faced 27

Tamarin, Moustached 27

Tamarin, Negro 27

Tamarin, Pied 26

Tamarin, Red-bellied 27

Tamarin, Red-chested 27

Tamarin, Red-handed 27

Tamarin, Rio Napo 27

Tamarin, Rufous-naped 27

Tamarin, Saddle-back 27

Tamarin, White-footed 27

Tamarin, White-lipped 27

Tamias 47

Tamiasciurus 47

tano, Tupaia 20

tansaniano, Crocidura 9

(tantalus, Cercopithecus) 31

(lao, Oryx) 101

Tapir, Andean 89

Tapir, Baird's 88

Tapit, Brazilian 89

Tapir, Central American 88

Tapir, Lowland 89

Tapir, Malayan 88

Tapir, Mountain 89

Tapir, South American 89

Tapir, Woolly 89

Tapirus 88-89

cardigradus, Loris 23

Tarsier, Eastern 25

Tarsier, Lesser Spectral 25

Tarsier, Philippine 25

Tarsier, Pygmy 25

Tarsier, Spectral 25

Tarsier, Sulawesi 25
Tarsier, Westem 25

Tarsius 25

Taruca 93

Tasmacetus 58

(tasmanicus, Arclocephalus) 85

tatauay, Cabassous 43

Latrica, Rupicapra rupicapra 102

tattersalli, Propithecus 23

(Taurotragus) 102

taxicolor, Budorcas 97

Tayassu 90

selfordi, Crocidura 9

telmalestes, Conepatus mesoleucus 71

Tembadau 96

temmincki, Felis 82

temmincki, Pzeropus 15

temminclai, Colobus badius 34

remmincki, Manis 44

(temminckii, Photoginus) 44

(temminckii, Procolobus) 34

Tenrec, Aquatic 5

Tenrec, Eastem Large-eared 5

Tenrec, Large-eared 5

Tenrec, Web-footed 5

Tenrec, Web-footed 5

(eephrosceles, Colobus badius) 35

tephrosceles, Colobus pennanni 35

(tephrosceles, Procolobus rufomitratus) 35

Teporingo 45

terrestris, Tapinus 89

Tetracerus 102

tetradoctyla, Manis 44

(tetradactyla, Phataginus) 44

tetradactyla, Tamandua 42

teuszii, Sousa 62

Thailand Brow-antlered Deer 92

(Thalarctos) 70

thalia, Crocidura 9

Thamin 92

Theropithecus 40

thibelana, Macaca 37

thibetanus, Selenarcios 69

(thibetanus, Ursus) 69

(tholloni, Colobus badius) 35

tholloni, Colobus pennantii 35

(tholloni, Procolobus rufomitratus) 35

(thomasi, Cercopitheus) 32

thomasi, Galago demidovii 24

(thomesi, Galago) 24

(thomesi, Galagoides demidoff) 24

thomasi, Microgale 6

thomasi, Presbytis 40

(thomasi, Presbyris comaza) 40

thomensis, Crociduro poensis 9

(thomensis, Crocidura) 9

Thomomys 48

thonglangyai, Craseonycteris 16

(thoracatus, Geocopromys) 55

thous, Dusicyon 67

Thylacine 1

Thylacinus 1

ribetana, Budorcas taxicolor 97

Tiger 84

Tiger, Deer 80

Tiger, Red 80

nigrina, Felis 82

tigris, Panthero 84

Titi, Dusky 29

Titi, Masked 29 
Titi, Widow 29

tokudae, Pieropus 15

Tokudaia 53

Tolypeutes 43

tonganus, Pleropus 15

tonkeana, Macaca 37

tora, Alcelaphus buselaphus 95

torquatus, Bradypus 43

torquatus, Callicebus 29

torquatus, Cercocebus 31

townsendi, Arctocephalus 85

townsendii, Plecotus 18

(Trachypithecus) $38-40$

Tragelaphus 102

(Tragelophus) 96

Tree-kangaroo, Bennett's 3

Tree-kangaroo, Black 4

Tree-kangaroo, Grizzled 3

Tree-kangaroo, Highland Unicolored 3

Tree-kangaroo, Lumholv' 4

Tree-kanganoo, Vogelkop 4

Tree-rat, Mimic 53

Tree-rat, Rabbit-eared 51

Tree-rat, White-footed 51

Treeshrew, Bornean Smooth-tailed 19

Treeshrew, Common 19

Treeshrew, Javan 19

Treeshrew, Large 20

Treeshrew, Lesser 19

Treeshrew, Madras 19

Treeshrew, Mountain 19

Treeshrew, Nicobar 19

Treeshrew, Northem Smooth-tailed 19

Treeshrew, Painted 20

Treeshrew, Palawan 19

Treeshrew, Pen-tailed 19

Treeshrew, Philippine 20

Treeshrew, Pygmy 19

Treeshrew, Ruddy 20

Treeshrew, Slender 19

Treeshrew, Striped 19

Tremarcios 69

trevelyani, Chrysaspalax 7

Trichechus 87

trichopus, Zygogeomys 48

trichotis, Allocebus 22

(trichatis, Cheirogaleus) 22

tricinctus, Tolypeutes 43

tricuspis, Manis 44

(tricuspis, Phataginus) 44

iridactyla, Mymecophaga 42

trilineata, Arctogalidia trivirgata 75

(tripartitus, Saguinus) 27

trissyllepsis. Peramyscus polionotus 50

trivirgatus, Aotus 28

traglodytes, Pan 42

(tropica, Bettongia) 3

tropicalis, Arctocephalus 85

tropicalis, Delphinus 59

(tropicalis, Delphinus delphis) 59

iropicalis, Monachus 86

truei, Podogymnura 7

truncatus, Chlamyphorus 43

truncatus, Tursiops 63

Tsessebe 99

tsuensis, Martes melampus 73

tuberculata, Mystacina 18

tuberculatus, Pueropus 16

Tucuxi 62
Tupaia 19-20

Tursiops 63

(Tursiops) 63

(Tympanoctomys) 55

tytonis, Chlorotalpa 7

Uaddan 95

Uakari. Bald 29

Uakari, Black 29

Uakari, Black-headed 29

Uakari, Red-and-white 29

uncia, Panthera 84

unicomis, Rhinoceros 89

Urial 101

Urocyon 68

Urogale 20

ursinus, Dendrolagus 4

ursinus, Melursus 69

ursinus, Papio hamadryas 38

(ursinus, Papio) 38

Ursus $69-70$

(Ursus) 69

urva, Herpestes 77

usambarae, Crocidura 9

(ustus, Saimin) 30

validus, Dendrahyrax 86

Vampire, Australian False 16

Vampyrops 17

vampyrus, Preropus 16

vancouverensis, Marmola 47

vanzolinii, Saimin 30

Vaquita 64

Varecia 22

variani, Hipporragus niger 100

variegata, Varecia 22

variegalus, Bradypus 43

(variegatus, Lemur) 22

(vellerasus, Calobus) 35

Venado 95

venaticus, Acinonyx jubarus 78

venatirus, Speothos 68

verreauxi, Propithecus 23

verrucosus, Sus 90

verus, Colabus 35

verus, Pan troglodytes $\mathbf{4 2}$

(verus, Procolobus) 35

vestitus, Phalanger 2

vetulus, Dusicyon 67

(vetulus, Presbyris) 40

vetulus, Preropus 16

(velulus, Trachypithecus) 40

vexillifer, Lipotes 56

vicugna, Vicugna 9 !

Vicugna 91

vignei, Ovis 101

(vignei, Ovis orientalis) 101

(villasa, Alaualta) 28

villosus, Chrysospalax 7

visagiei, Chrysachloris 7

viltata, Galictis 72

vitticallis, Herpestes 77

Viverra 76-77

(Viverra) 75

Viverriculo 77

viverrina, Felis 82

(vociferans, Aotus) 28

yoelizkowi, Pleropus 16

Vole, Amargosa 51

Vole, Beach 51

Vole, Chihuahua 51
Vole, Florida Saltmarsh 51

(vomerina, Phocoena) 63

Vormela 75

Vulpes 68

(Vulpes) 68

wagneri, Calagonus 90

waldroni, Colobus bodius 34

(waldroni, Procolobus badius) 34

walie, Capra ibex 97

(walie, Capra) 97

Wallaby, Black Dorcopsis 4

Wallaby, Bridled Nailtai] 4

Wallaby, Crescent Nailtail 4

Wallaby, Toolache 4

wallichi, Cervus elaphus 92

Walrus 85

Walrus, Laptev 85

Wambenger, Red-Lailed 1

Water-rat, False 53

Weasel, Indonesian Mountain 74

Whale, Andrews' Beaked 57

Whale, Arch-beaked 57

Whale, Arnoux's Beaked 56

Whale, Baird's Beaked 56

Whale, Black Right 65

Whale. Blainville's Beaked 57

Whale, Blue 65

Whale, Bowhead 66

Whale, Bryde's 64

Whale, Dwarf Sperm 58

Whale, False Killer 61

Whale, Fin 65

Whale, Gervais' Beaked 57

Whale, Giant Bottle-nosed 56

Whale. Ginkgo-toothed Beaked 57

Whale, Goose-beaked 58

Whale, Gray's Beaked 57

Whale, Greenland Right 66

Whale, Grey 64

Whale, Gulf Stream Beaked 57

Whale, Hector's Beaked 57

Whale, Hubbs' Beaked 57

Whale, Humpback 65

Whale, Indo-pacific Beaked 56

Whale, Indo-pacific Beaked 56

Whale, Killer 61

Whale, Layard's Beaked 57

Whale, Long-finned Pilot 60

Whale, Longman's Beaked 56

Whale. Melon-headed 61

Whale, Northern Four-toothed 56

Whale, Northern Right 61

Whale, Pacific Pilot 60

Whale, Pygmy Killer 59

Whale, Pygmy Right 66

Whale, Pygmy Sperm 58

Whale, Sei 64

Whale, Shepherd's Beaked 58

Whale, Shon-finned Pilot 60

Whale, Skew-beaked 57

Whale, Southern Bottenose 56

Whale, Southem Four-loothed 56

Whale, Southern Right 61

Whale, Sowerby's Beaked 57

Whale, Sperm 58

Whale, Splaytooth Beaked 57

Whale, Stejneger's Beaked 58

Whale, Strap-toothed 57

Whale, Sulphur-bottom 65 
Whale, Tasman 58

Whale, True's Beaked 57

Whale, Unicom 59

Whale, White 58

wiedii, Felis 83

wimmeri, Crocidura 9

wintoni, Cryptochloris 7

Wisent 96

Wolf, Abyssinian 67

Wolf, Andean 67

Wolf, Maned 67

Wolf, Red 67

Wolf, Tasmanian 1

wolfi, Cercopithecus 33

(wolfi, Cercopithecus pogonias) 33

Wolverine 72

Wombat, Northem Hairy-nosed 3

Wombat, Queensland Hairy-nosed 3

woodfordi, Pteropus 16
Woodrat, Key Largo 49

Woodrat, San Joaquin Valley 49

Woylie 3

Wurrung 4

Xenuromys 53

Xeromys 53

yagouaroundi, Felis 83

Yak, Wild 96

yakui, Macaca fiscato 36

Yallara 2

yarkandensis, Cervus elaphus 92

yerbabuenae, Leptonycteris 17

Zoglossus 1

Zalophus 85

zanzibaricus, Galago 25

(zanzibaricus, Galago senegalensis) 25

Zapus 53

zebra, Cephalophus 98

zebro, Equus zebro 88
Zebra, Cape Mountain 88

Zebra, Grevy's 88

Zebra, Hartmann's Mountain 88

(zerdo, Fennecus) 68

zerda, Vulpes 68

zibetha, Viverro 77

zimmermanni, Crocidura 9

Ziphins 58

Zorro, Azara's 67

Zorro, Common 67

Zorro, Grey 67

Zorro, Hoary 67

Zorro, Secburan 67

Zorro, Small-eared 67

Zygogeomys 48

zyli, Cryplochloris 7

Zyzomys 53 

The UK Joint Nature Conservation Committee was established by the Environmental Protection Act 1990 "for the purposes of nature conservation, and fostering the understanding thereof" in Great Britain as a whole and outside Great Britain. It is a committee of the three country agencies (the Countryside Council for Wales, English Nature and Scottish Natural Heritage), together with independent members and representatives from Northern Ireland and the Countryside Commission, and is supported by a specialist staff. JNCC and the three country agencies carry forward duties previously undertaken by the Nature Conservancy Council.

JNCC's statutory responsibilities include:

- the establishment of common scientific standards;

- the undertaking and commissioning of research;

- advising Ministers on the development and implementation of policies for or affecting nature conservation for Great Britain as a whole or nature conservation outside Great Britain;

- the provision of advice and dissemination of knowledge to any persons about nature conservation.

JNCC also has the UK responsibility for European and international matters affecting nature conservation.

Details of publications produced by JNCC are available from JNCC, Monkstone House, City Road, Peterborough PE1 1 JY. 